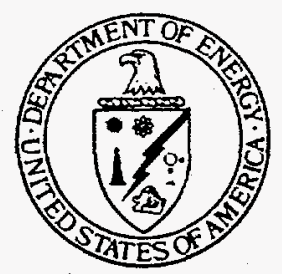

Department of Energy

Oak Ridge Operations

Weldon Spring Site

Remedial Action Project Office

7295 Highway 94 South

St. Charles, Missouri 63304

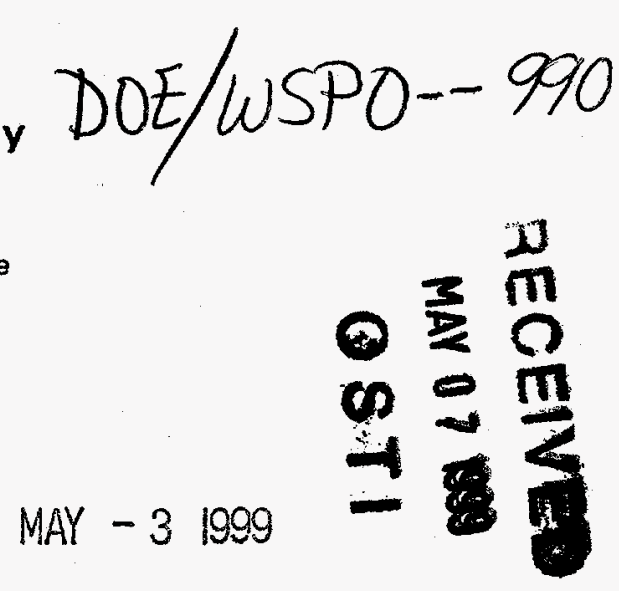

DISTRIBUTION :

\title{
QUARTERLY ENVIRONMENTAI DATA SUMMARY FOR FIRST QUARTER 1999
}

In support of the Weldon Spring Site Remedial Action Project Federal Facilities Agreement, a copy of the Quarterly

Environmental Data Summary (QEDS) for the first quarter of 1999 is enclosed.

The data presented in this letter and enclosure constitute the QEDS. The data, except for air monitoring data and site KPA generated data (uranium analyses), were received from the contract laboratories, verified by the Weldon spring site verification group and merged into the database during the first quarter of 1999. KPA results for on-site total uranium analyses performed during first quarter 1999 are included. Air monitoring data presented are the most recent complete sets of quarterly data.

Significant data, defined as data values that have exceeded defined "above normal" Level 2 values, are discussed in this letter for Environmental Monitoring Plan (EMP) generated data only. Above normal Level 2 values are based, in ES\&H procedures, on historical high values, DOE Derived Concentration Guides (DCGS), NPDES limits, and other guidelines. The procedures also establish actions to be taken in the event that "above normal" data occur.

All data received and verified during the first quarter were within a permissible range of variability except for those detailed below. Above normal occurrences are cited for groundwater, air, and NPDES data. There were none for springs or surface water. The following discussion offers a brief summary of the data merged during the quarter that exceeded the above normal criteria and updates on past reported above-normal data. The enclosed tables present the most recent data for air and the data merged into the database during the first quarter 1999 for groundwater, NPDES, surface water, and springs.

Graphs showing concentrations of selected contaminants of concern at some of the critical locations have also been included in this QEDS. The graphs are discussed in the separate sections. 


\section{DISCLAIMER}

This report was prepared as an account of work sponsored by an agency of the United States Government. Neither the United States Government nor any agency thereof, nor any of their employees, make any warranty, express or implied, or assumes any legal liability or responsibility for the accuracy, completeness, or usefulness of any information, apparatus, product, or process disclosed, or represents that its use would not infringe privately owned rights. Reference herein to any specific commercial product, process, or service by trade name, trademark, manufacturer, or otherwise does not necessarily constitute or imply its endorsement, recommendation, or favoring by the United States Government or any agency thereof. The views and opinions of authors expressed herein do not necessarily state or reflect those of the United States Government or any agency thereof. 


\section{DISCLAIMER}

Portions of this document may be illegible in electronic image products. Images are produced from the best available original document. 
Distribution

Page 2

\section{NPDES}

Uranium concentrations at the three major NPDES outfalls are graphed for January 1998 to March 1999. These graphs are located at the beginning of the NPDES tables. For comparison purposes, the annual averages for 1997 and 1998 are also graphed. The derived concentration guide (DCG) of $600 \mathrm{pCi} / 1$ for uranium may also be used for comparison. The DCG is the concentration of a radionuclide in air or water that, under conditions of continuous exposure for 1 year by one exposure mode (i.e., ingestion of water), would result in an effective dose equivalent of $100 \mathrm{mrem}$. A graph of historical annual averages (through March 1999) for the three outfalls is also enclosed.

Outfall NP-0002 was slightly above the 1998 annual average for uranium (22.3 pCi/l) for February, at $23.3 \mathrm{pCi} / 1$, and below average for the months of January and March. The 1999 annual average (to date) is $13.7 \mathrm{pCi} / 1$, which is lower than the 1998 average and can be attributed to natural variations (rainfall, etc). Most of the NP-0002 watershed has been remediated and uranium levels are expected to stay well below the preremediation levels.

The uranium levels at NP-0003 were above the 1998 average (83.1 $\mathrm{pCi} / 1)$ for the months of February and March. The 1999 annual average (to date) is 86.9, just slightly over the 1998 average. While most of the Outfall NP-0003 watershed has been remediated, it contains Ash Pond where contaminated soils, concrete, etc., were stored. Most of these materials have been placed in the disposal cell, and all that remains is what was in Ash Pond before remediation started. These remaining in situ materials are also in the process of being removed. This will cause uranium concentrations to fluctuate. Water is released from the Ash Pond area only if it is less than $600 \mathrm{pCi} / 1$ for uranium; however, it may still be higher than the previous Outfall NP-0003 annual average, which could cause an increase in the current annual average. The volume of runoff from Ash Pond is low in comparison to the remainder of the watershed.

Outfall NP-0004 was added to the NPDES permit during May of 1998 . The watershed that flows to the outfall includes the remediated section of Pit 4. The maximum uranium level for the 3-month period was $7.15 \mathrm{pCi} / 1$. Because NP-0004 is a small watershed, the uranium data are not graphed.

Outfall NP-0005 was above the 1998 annual average (10 pCi/1) for the 3 months of the quarter. The 1999 annual average (to date) is $19.7 \mathrm{pCi} / 1$, which is an increase from the 1998 average but still well below the DCG. Activity in the TSA may have contributed to the slight increase. A large portion of the NP0005 watershed has been remediated, and uranium levels are expected to remain well below pre-remediation levels. 
Outfall NP-0010, the CMSA sedimentation basin outfall, was slightly below the 1998 annual average $(10.7 \mathrm{pCi} / 1)$ for all 3 months. The area has been remediated and uranium levels are expected to remain low, but the use of a small area for vehicle maintenance may increase uranium levels slightly. Because NP0010 is a minor outfall, it is not graphed.

Outfall NP-1005, the new storm water outfall at the quarry, was in compliance with all permitted parameters. The maximum uranium level during the 3 -month period was $1.28 \mathrm{pCi} / 1$.

The above normals for data merged during the first quarter, and any above normals that were not resolved in previous QEDS are discussed below.

\section{NP-EPS2-030199}

Site Batch $\mathrm{S} 154$ was released based on process monitoring and a KPA uranium result. This is an acceptable practice that had been followed several times in the past. When the contract laboratory results were received, the selenium result was $55.6 \mathrm{\mu g} / \mathrm{l}$. The permitted limit is $50 \mu \mathrm{g} / \mathrm{I}$. A reanalysis by the original laboratory and analyses by several other laboratories of the parent sample confirmed the elevated level. All other parameters were in compliance. Process samples had indicated that selenium was present at a much lower level. An investigation was conducted, and the cause for the elevated level could not be pinpointed; however, operational changes have been made to improve confidence in process monitoring results. All batches subsequent to 154 were in compliance with selenium as well as all other parameters.

\section{GROUNDWATER}

\section{Chemical Plant}

\section{Site Water Treatment Plant and Temporary Storage Area}

One filtered groundwater sample from the TSA area exceeded baseline levels for barium. Total uranium baseline was slightly exceeded in one sample from the equalization basin area. On-site KPA results were used for uranium comparisons. No groundwater impacts from the site water treatment plant or TSA are suspected. 
- Sample \# GW-2039-B698

Barium (380 ug/l) was reported above baseline (240 ug/l) for the last bimonthly 1998 sampling. This location monitors the east side of the TSA. There are no other parameters out of normal range, which suggests TSA as the source of the excursion. Subsequent data will be used to determine whether an increasing barium trend is apparent at this location.

- Sample \# GW-2042-Q498

Total uranium for the fourth quarter 1998 (5.25 pCi/l) exceeded baseline (3.33 pCi/l). The on-site KPA analytical result for this sample was $3.07 \mathrm{pCi} / 1$, which is below baseline. Samples from this location monitor groundwater along the south side of the equalization basin. No other analytical results suggest impact from the basin, but subsequent samples from this location will be reviewed to determine if uranium is increasing.

\section{Disposal Cell Detection Monitoring}

The second semiannual groundwater detection-monitoring event was completed during December 1998. Most of the data for this sampling event are included in the tables of this report (detection monitoring wells are MW-2032 and MW-2045 through MW2048). Analytical results from this sampling event were statistically compared to the baseline data compiled during 1997 and early 1998. No groundwater contamination attributable to the disposal cell was identified. The analytical results and statistical analyses of the first and second semiannual RCRA detection monitoring are being presented in the 1998 annual site environmental report.

\section{WELDON SPRING QUARRY}

\section{Quarry Water Treatment Plant}

- Sample \# GW-1040-Q19

Elevated chloride conditions continued to be reported through the first quarter (24.4 mg/l) of 1999 (baseline is $16.0 \mathrm{mg} / \mathrm{l}$ ). Chloride is also elevated at the upgradient location for the quarry water treatment plant and the quarry is not suspected as the source of the excursion.

\section{Quarry Vicinity}

No above normal values or indications of any increasing contaminant concentrations were reported for the first quarter 1999. 


\section{St. Charles County Well Field}

No samples from the st. Charles County well field were reported

to have above normal values during the first quarter 1999. Total uranium values reported for the quarter are plotted on graphs (including the results from the previous four quarters) found at the front of the groundwater tables section.

\section{SURFACE WATER}

No surface water contaminants (at Level 2 above normal concentrations) were detected in samples collected during the first quarter of 1999.

\section{SPRINGS}

No elevated contaminants were reported in samples from springs during the first quarter of 1999. Total uranium values at the Burgermeister spring are plotted versus time on the figure attached in the front of the tables.

\section{AIR}

Data for the air monitoring locations are enclosed. Graphs of the monitoring results are located in front of the tables and are discussed below. There was one above normal air monitoring result from west of the TSA for the first quarter of 1999, which is highlighted in the following.

The gross alpha concentrations for radioactive air particulate monitoring for the first quarter 1999 are charted with the background level, which is the most recent 52 -week average. Background plus three standard deviations is shown for comparison. The background location is AP-4012, at the Daniel Boone Elementary School in New Melle.

Environmental TLD monitoring results for the fourth quarter 1998 are charted with the annual background result and the background plus 25 mrem effective dose equivalent (EDE) shown for comparison. The $25 \mathrm{mrem} E D E$ is based on one-fourth of the annual 100 mrem EDE limit for the public established in DOE Order 5400.5. The background locations are TD-4005 (west of the Army site) and TD-4009 (Daniel Boone Elementary School, New Melle).

The alpha track radon and thoron monitoring results are graphed for the fourth quarter of 1998. The background level (based on the most recent four quarters of monitoring), and the derived concentration guides (DCG) for radon and thoron are shown for comparison. DCGs are reference values established for protection of the public and the environment and are listed in DOE Order 5400.5 .

The background locations are RD-4005 (west of the Army site) and RD-4009 (Daniel Boone Elementary School, New Melle). 


\section{Raffinate Pit 4 and TSA}

AP-3004-WK1399

Air samples collected at radioactive airborne particulate monitor AP-3004, located immediately west of the TSA, exceeded the 52week average background concentration plus 3 standard deviations for the period March 17-31, 1999, which triggered the above normal reporting process. The weekly monitoring results at this station were $6.44 \mathrm{E}-15$ and $6.04 \mathrm{E}-15 \mu \mathrm{Ci} / \mathrm{ml}$ for weeks 12 and 13 , respectively. The 52-week background concentration as of March 13 was $1.26 \mathrm{E}-15 \mu \mathrm{Ci} / \mathrm{ml}$. The elevated concentrations were attributed to raffinate sludge treatment and storage within Raffinate Pit 3 and the TSA. A conservative dose assessment based on the week 12 concentration and an assumed exposure time of 2,000 hours gave a maximum dose equivalent of 4.7 mrem to a hypothetical individual located at the monitoring station. The site perimeter is continuously monitored by low volume air monitors for emissions of radioactive airborne particulates.

\section{SUMMARY}

The analytical results highlighted in the preceding sections of this document were reported as being above prescribed baseline values, varying from historical ranges or being above regulatory limits, and as a result, are subject to more focused attention by the WSSRAP Environmental Protection Group. Continued monitoring is performed to determine the need for additional investigation or action. All other data subject to reporting in the QEDS were within historic range or below reporting criteria unless highlighted in the above discussion.

If you have any questions, please contact the WSSRAP Community Relations Department at (314)441-8086.

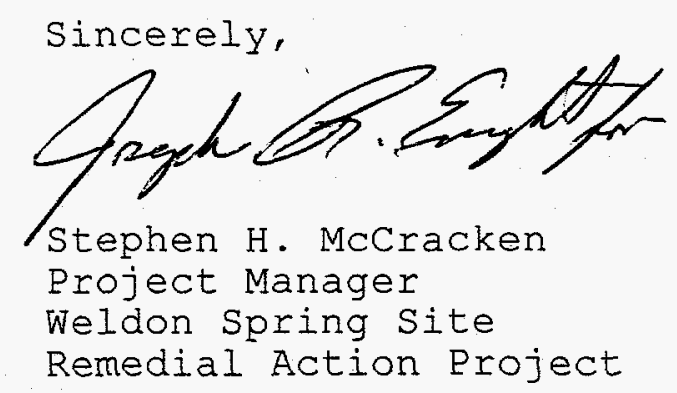

Enclosure:

As stated 


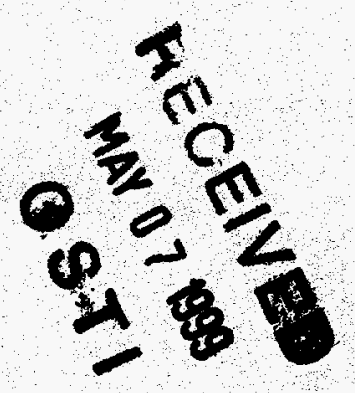

NPDES 


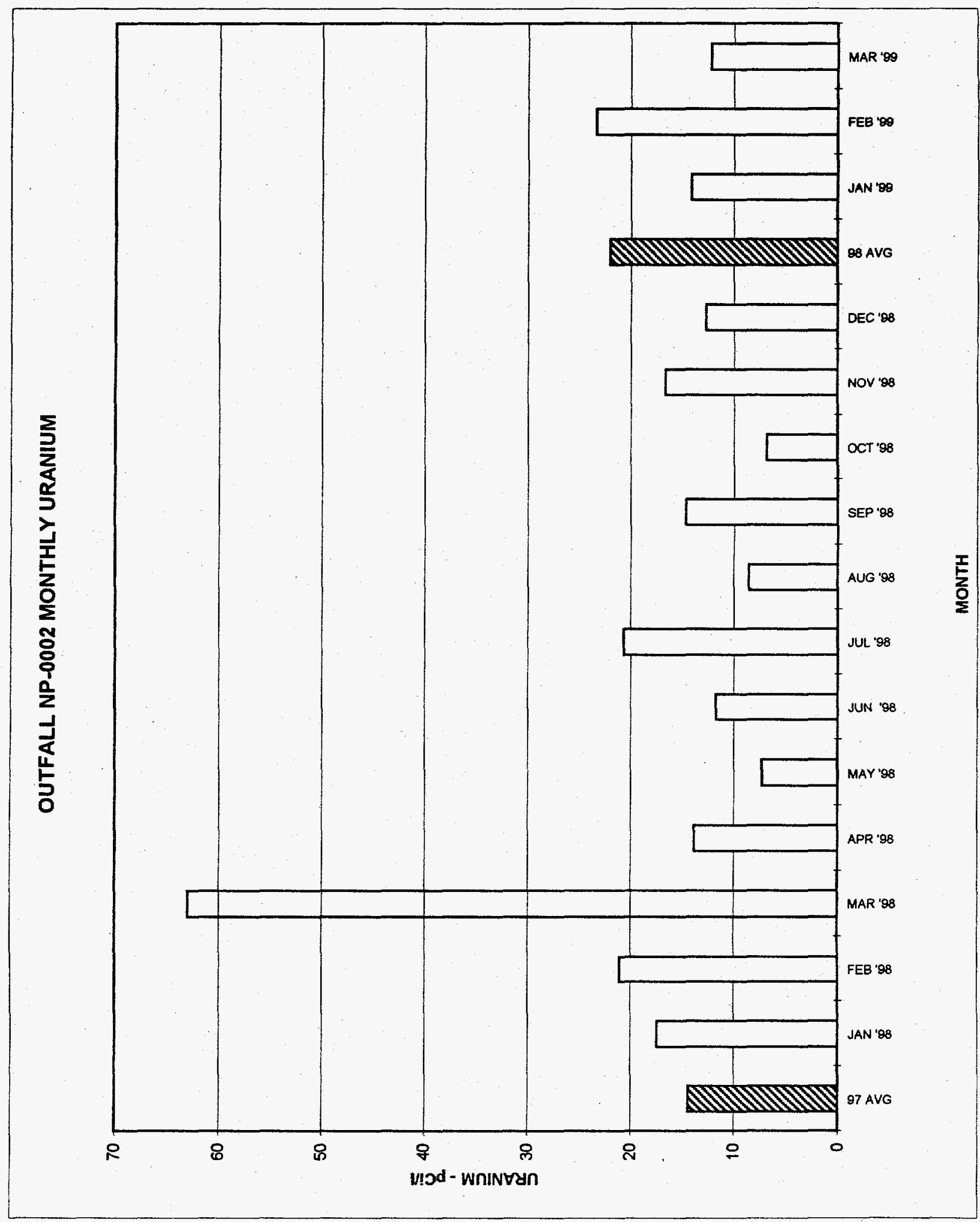




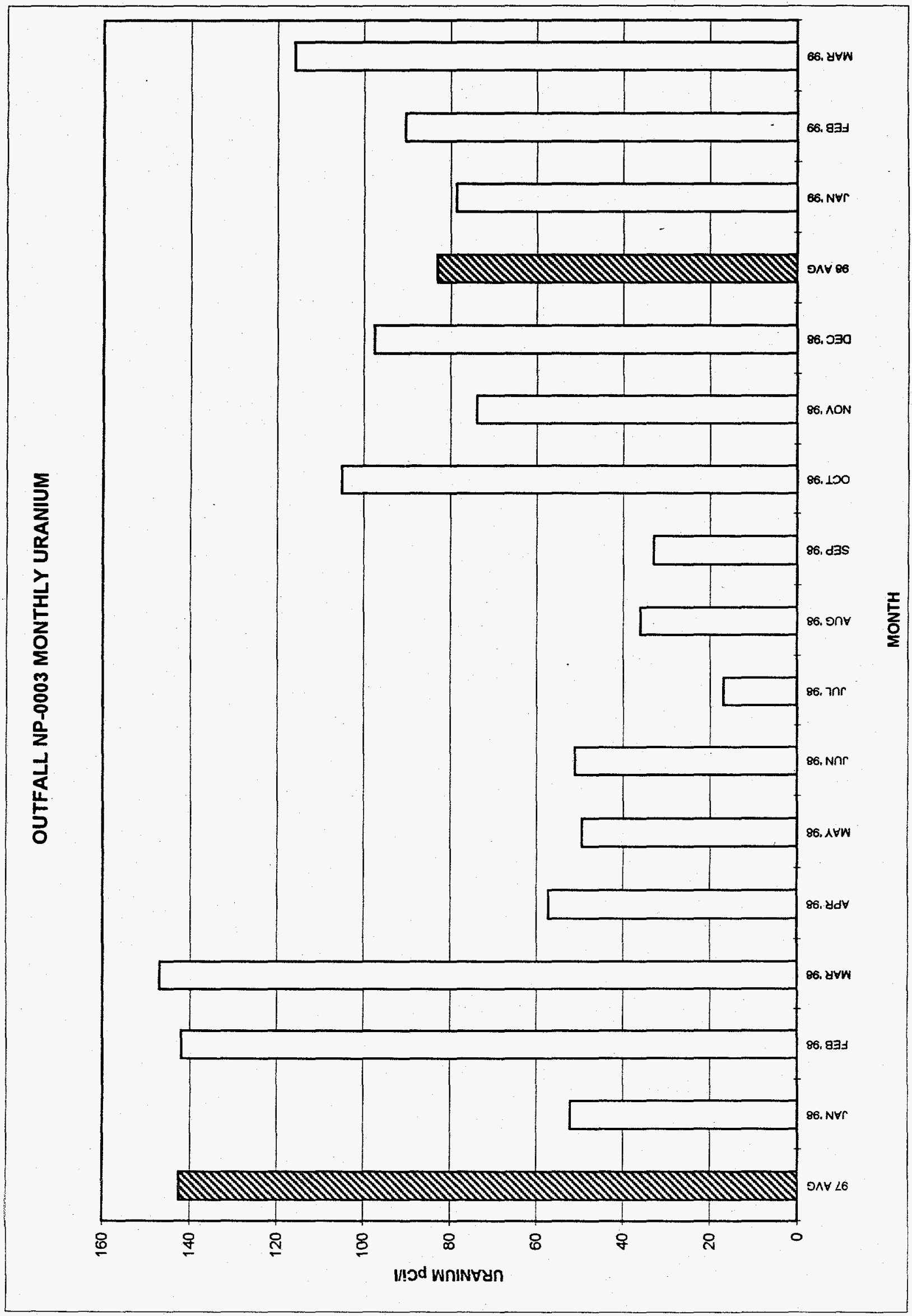




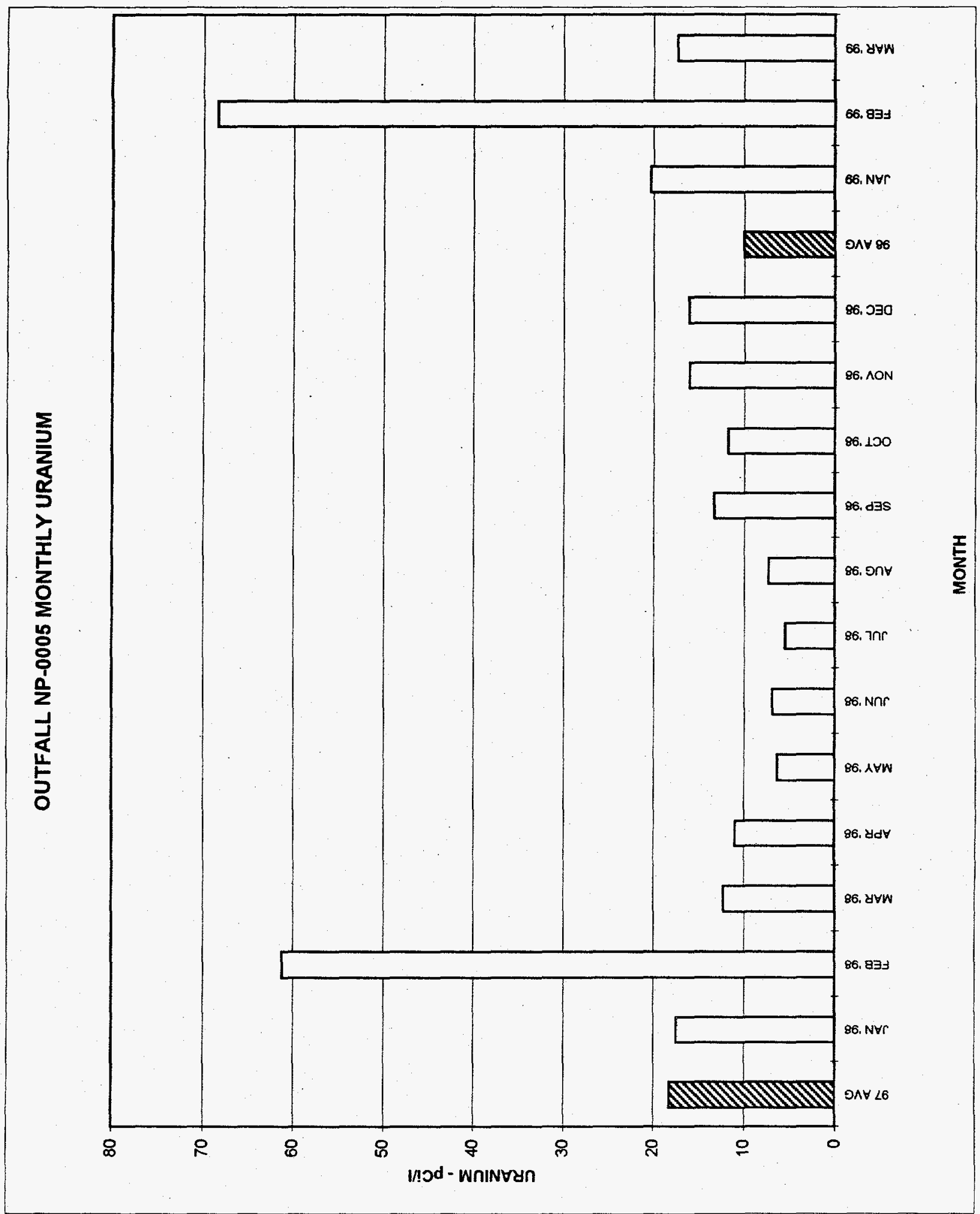




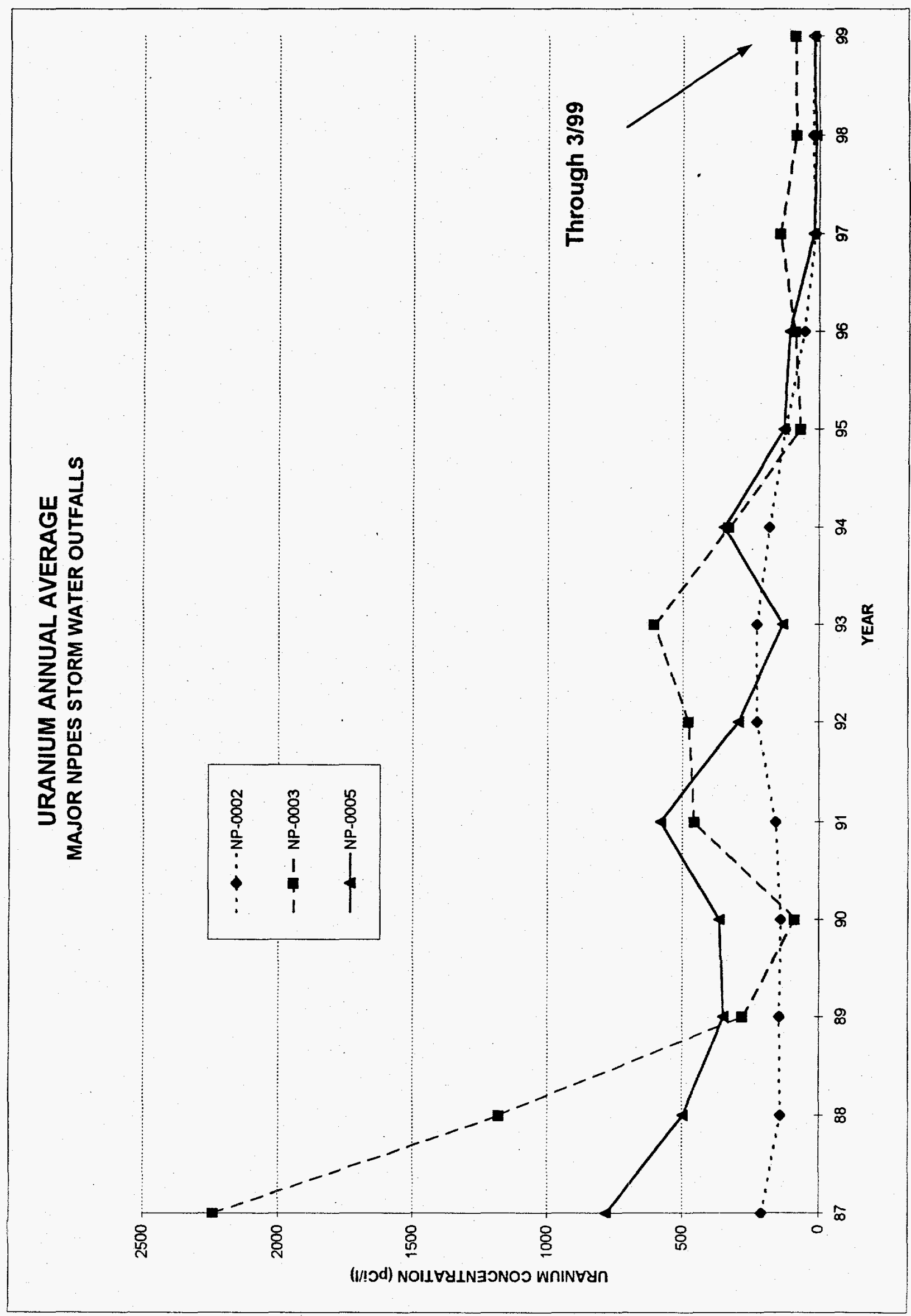


NPDES

DATA MERGED DURING FIRST QUARTER, 1999

\begin{tabular}{|c|c|c|c|c|c|c|}
\hline WSSRAP_ID & DATE_SAM & PARAMETER & CONC & $\mathrm{DL}$ & UNITS & METHCD \\
\hline 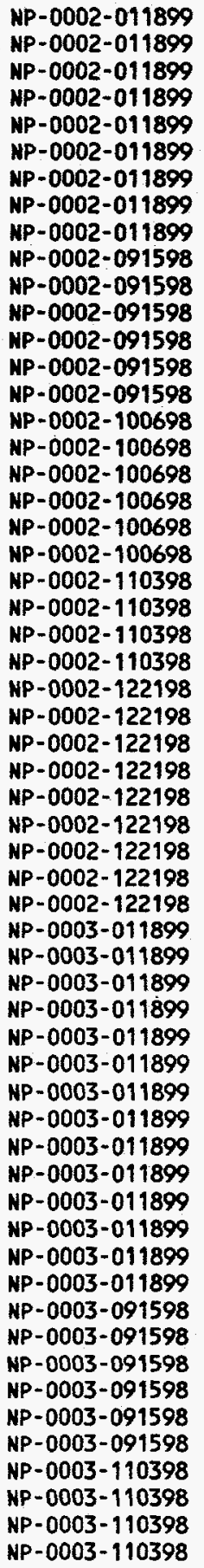 & $\begin{array}{l}01 / 18 / 99 \\
01 / 18 / 99 \\
01 / 18 / 99 \\
01 / 18 / 99 \\
01 / 18 / 99 \\
01 / 18 / 99 \\
01 / 18 / 99 \\
01 / 18 / 99 \\
01 / 18 / 99 \\
09 / 15 / 98 \\
09 / 15 / 98 \\
09 / 15 / 98 \\
09 / 15 / 98 \\
09 / 15 / 98 \\
09 / 15 / 98 \\
10 / 06 / 98 \\
10 / 06 / 98 \\
10 / 06 / 98 \\
10 / 06 / 98 \\
10 / 06 / 98 \\
10 / 06 / 98 \\
11 / 03 / 98 \\
11 / 03 / 98 \\
11 / 03 / 98 \\
11 / 03 / 98 \\
12 / 21 / 98 \\
12 / 21 / 98 \\
12 / 21 / 98 \\
12 / 21 / 98 \\
12 / 21 / 98 \\
12 / 21 / 98 \\
12 / 21 / 98 \\
12 / 21 / 98 \\
12 / 21 / 98 \\
01 / 18 / 99 \\
01 / 18 / 99 \\
01 / 18 / 99 \\
01 / 18 / 99 \\
01 / 18 / 99 \\
01 / 18 / 99 \\
01 / 18 / 99 \\
01 / 18 / 99 \\
01 / 18 / 99 \\
01 / 18 / 99 \\
01 / 18 / 99 \\
01 / 18 / 99 \\
01 / 18 / 99 \\
01 / 18 / 99 \\
09 / 15 / 98 \\
09 / 15 / 98 \\
09 / 15 / 98 \\
09 / 15 / 98 \\
09 / 15 / 98 \\
09 / 15 / 98 \\
11103 / 98 \\
11 / 03 / 98 \\
11 / 03 / 98 \\
11 / 03 / 98\end{array}$ & $\begin{array}{l}\text { NITRATE-N } \\
\text { ARSENIC } \\
\text { CHROMIUM } \\
\text { LEAD } \\
\text { THALIIUM } \\
\text { TOTAL SUSPENDED SOLIDS } \\
\text { GROSS ALPHA } \\
\text { GROSS BETA } \\
\text { URANIUM, TOTAL } \\
\text { NITRATE-N } \\
\text { ARSENIC } \\
\text { CHROMIUM } \\
\text { LEAD } \\
\text { THALLIUM } \\
\text { TOTAL SUSPENDED SOLIDS } \\
\text { NITRATE-N } \\
\text { ARSENIC } \\
\text { CHROMIUM } \\
\text { LEAD } \\
\text { THALLIUM } \\
\text { TOTAL SUSPENDED SOLIDS } \\
\text { ARSENIC } \\
\text { CHROMIUM } \\
\text { LEAD } \\
\text { THALLIUM } \\
\text { NITRATE-N } \\
\text { ARSENIC } \\
\text { CHROMIUM } \\
\text { LEAD } \\
\text { THALLIUM } \\
\text { TOTAL SUSPENDED SOLIDS } \\
\text { GROSS ALPHA } \\
\text { GROSS BETA } \\
\text { URANIUM, TOTAL } \\
\text { NITRATE-N } \\
\text { ARSENIC } \\
\text { CHROMIUM } \\
\text { LEAD } \\
\text { THALLIUM } \\
\text { TOTAL SUSPENDED SOLIDS } \\
\text { GROSS ALPHA } \\
\text { GROSS BETA } \\
\text { RADIUM-226 } \\
\text { RADIUM-228 } \\
\text { THORIUM-228 } \\
\text { THORIUM-23O } \\
\text { THORIUM-232 } \\
\text { URANIUM, TOTAL } \\
\text { NITRATE-N } \\
\text { ARSENIC } \\
\text { CHROMIUM } \\
\text { LEAD } \\
\text { THALLIUM } \\
\text { TOTAL SUSPENDED SOLIOS } \\
\text { ARSENIC } \\
\text { CHROMIUM } \\
\text { LEAD } \\
\text { THALLIUM } \\
\text { THE }\end{array}$ & 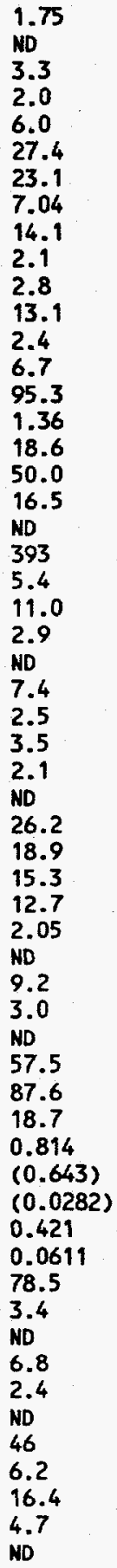 & 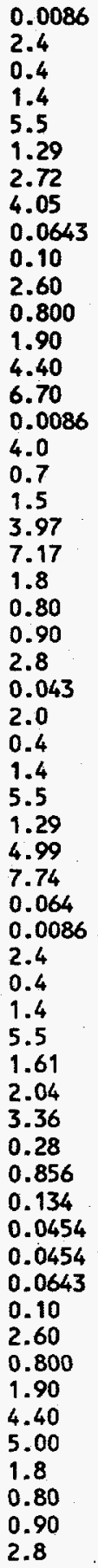 & $\begin{array}{l}M G / L \\
U G / L \\
U G / L \\
U G / L \\
U G / L \\
M G / L \\
P C I / L \\
P C I / L \\
P C I / L \\
M G / L \\
U G / L \\
U G / L \\
U G / L \\
U G / L \\
M G / L \\
M G / L \\
U G / L \\
U G / L \\
U G / L \\
U G / L \\
M G / L \\
U G / L \\
U G / L \\
U G / L \\
U G / L \\
M G / L \\
U G / L \\
U G / L \\
U G / L \\
U G / L \\
M G / L \\
P C I / L \\
P C I / L \\
P C I / L \\
M G / L \\
U G / L \\
U G / L \\
U G / L \\
U G / L \\
M G / L \\
P C I / L \\
P C I / L \\
P C I / L \\
P C I / L \\
P C I / L \\
P C I / L \\
P C I / L \\
P C I / L \\
M G / L \\
U G / L \\
U G / L \\
U G / L \\
U G / L \\
M G / L \\
U G / L \\
U G / L \\
U G / L \\
U G / L \\
\end{array}$ & $\begin{array}{l}\text { EPA } 353.1 \\
\text { EPA CLP } \\
\text { EPA CLP } \\
\text { EPA CLP } \\
\text { EPA CLP } \\
\text { EPA } 160.2 \\
\text { EPA } 900.0 \\
\text { EPA } 900.0 \\
\text { ASTM } 5174-91 \\
\text { EPA } 353.1 \\
\text { EPA CLP } \\
\text { EPA CLP } \\
\text { EPA CLP } \\
\text { EPA CLP } \\
\text { EPA } 160.2 \\
\text { EPA } 353.1 \\
\text { EPA } 6010 A \\
\text { EPA } 6010 A \\
\text { EPA } 6010 A \\
\text { EPA } 6010 A \\
\text { EPA } 160.2 \\
\text { EPA CLP } \\
\text { EPA CLP } \\
\text { EPA CLP } \\
\text { EPA CLP } \\
\text { EPA } 353.1 \\
\text { EPA CLP } \\
\text { EPA CLP } \\
\text { EPA CLP } \\
\text { EPA CLP } \\
\text { EPA } 160.2 \\
\text { EPA } 900.0 \\
\text { EPA } 900.0 \\
\text { ASTM } 5174-91 \\
\text { EPA } 353.1 \\
\text { EPA CLP } \\
\text { EPA CLP } \\
\text { EPA CLP } \\
\text { EPA CLP } \\
\text { EPA } 160.2 \\
\text { EPA } 900.0 \\
\text { EPA } 900.0 \\
\text { EPA } 903.1 \\
\text { EPA } 904.0 \\
\text { HASL } 300 \\
\text { HASL } 300 \\
\text { HASL } 300 \\
\text { ASTM } 5174-91 \\
\text { EPA } 353.1 \\
\text { EPA CLP } \\
\text { EPA CLP } \\
\text { EPA CLP } \\
\text { EPA CLP } \\
\text { EPA } 160.2 \\
\text { EPA CLP } \\
\text { EPA CLP } \\
\text { EPA CLP } \\
\text { EPA CLP }\end{array}$ \\
\hline
\end{tabular}




\begin{tabular}{|c|c|c|c|c|c|c|}
\hline WSSRAP_10 & DATE_SAM & PARAMETER & CONC & DL & UNITS & METHOD \\
\hline 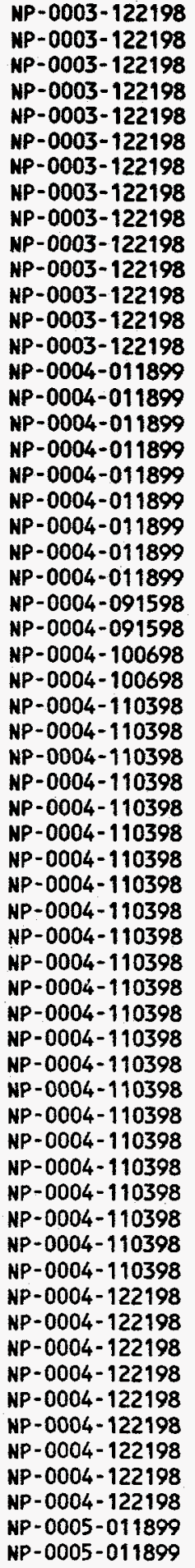 & $\begin{array}{l}12 / 21 / 98 \\
12 / 21 / 98 \\
12 / 21 / 98 \\
12 / 21 / 98 \\
12 / 21 / 98 \\
12 / 21 / 98 \\
12 / 21 / 98 \\
12 / 21 / 98 \\
12 / 21 / 98 \\
12 / 21 / 98 \\
12 / 21 / 98 \\
12 / 21 / 98 \\
12 / 21 / 98 \\
12 / 21 / 98 \\
01 / 18 / 99 \\
01 / 18 / 99 \\
01 / 18 / 99 \\
01 / 18 / 99 \\
01 / 18 / 99 \\
01 / 18 / 99 \\
01 / 18 / 99 \\
01 / 18 / 99 \\
01 / 18 / 99 \\
09 / 15 / 98 \\
09 / 15 / 98 \\
10 / 06 / 98 \\
10 / 06 / 98 \\
11 / 03 / 98 \\
111 / 03 / 98 \\
111 / 03 / 98 \\
11 / 03 / 98 \\
11 / 03 / 98 \\
11 / 03 / 98 \\
11 / 03 / 98 \\
111 / 03 / 98 \\
11 / 03 / 98 \\
11 / 03 / 98 \\
11103 / 98 \\
111 / 03 / 98 \\
11 / 03 / 98 \\
11 / 03 / 98 \\
11 / 03 / 98 \\
11 / 03 / 98 \\
111 / 03 / 98 \\
11 / 03 / 98 \\
11 / 03 / 98 \\
11 / 03 / 98 \\
11 / 03 / 98 \\
111 / 03 / 98 \\
11 / 03 / 98 \\
12 / 21 / 98 \\
12 / 21 / 98 \\
12 / 21 / 98 \\
12 / 21 / 98 \\
12 / 211 / 98 \\
12 / 21 / 98 \\
12 / 21 / 98 \\
12 / 21 / 98 \\
12 / 21 / 98 \\
01 / 18 / 99 \\
01 / 18 / 99\end{array}$ & $\begin{array}{l}\text { NITRATE-N } \\
\text { ARSENIC } \\
\text { CHROMIUM } \\
\text { LEAD } \\
\text { THALLIUM } \\
\text { TOTAL SUSPENDED SOLIOS } \\
\text { GROSS ALPHA } \\
\text { GROSS BETA } \\
\text { RADIUM-226 } \\
\text { RADIUM-228 } \\
\text { THORIUM-228 } \\
\text { THORIUM-230 } \\
\text { THORIUM-232 } \\
\text { URANIUM, TOTAL } \\
\text { NITRATE-N } \\
\text { ARSENIC } \\
\text { CHROMIUM } \\
\text { LEAD } \\
\text { THALLIIM } \\
\text { TOTAL SUSPENDED SOLIDS } \\
\text { GROSS ALPHA } \\
\text { GROSS BETA } \\
\text { URANIUM, TOTAL } \\
\text { NITRATE-N } \\
\text { TOTAL SUSPENDED SOLIDS } \\
\text { NITRATE-N } \\
\text { TOTAL SUSPENDED SOLIDS } \\
\text { ALUMINUM } \\
\text { ANTIMONY } \\
\text { ARSENIC } \\
\text { BARIUM } \\
\text { BERYLIUM } \\
\text { CADMIUM } \\
\text { CALCIUM } \\
\text { CHROMIUM } \\
\text { COBALT } \\
\text { COPPER } \\
\text { IRON } \\
\text { LEAD } \\
\text { MAGNESIUM } \\
\text { MANGANESE } \\
\text { MERCURY } \\
\text { NICKEL } \\
\text { POTASSIUM } \\
\text { SELENIUM } \\
\text { SILVER } \\
\text { SODIUM } \\
\text { THALLIUM } \\
\text { VANADIUM } \\
\text { ZINC } \\
\text { NITRATE-N } \\
\text { ARSENIC } \\
\text { CHRONIUM } \\
\text { LEAD } \\
\text { THALLIUM } \\
\text { TOTAL SUSPENDED SOLIDS } \\
\text { GROSS ALPHA } \\
\text { GROSS BETA } \\
\text { URANIUM, TOTAL } \\
\text { NITRATE-N } \\
\text { TOTAL SUSPENDED SOLIDS }\end{array}$ & 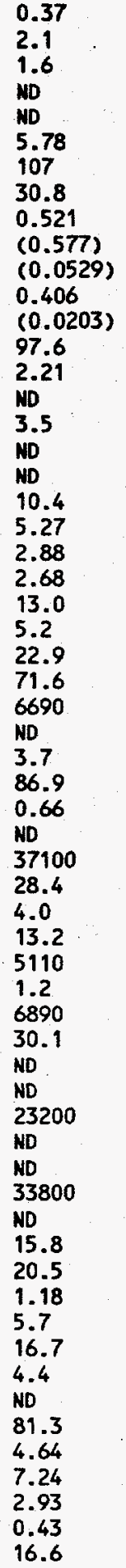 & 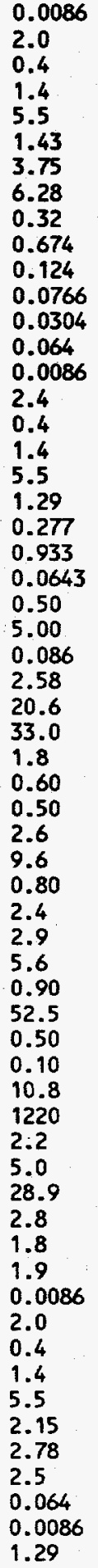 & 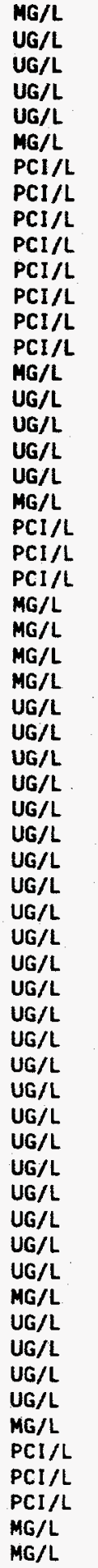 & $\begin{array}{l}\text { EPA } 353.1 \\
\text { EPA CLP } \\
\text { EPA CLP } \\
\text { EPA CLP } \\
\text { EPA CLP } \\
\text { EPA } 160.2 \\
\text { EPA } 900.0 \\
\text { EPA } 900.0 \\
\text { EPA } 903.1 \\
\text { EPA } 904.0 \\
\text { HASL } 300 \\
\text { HASL } 300 \\
\text { HASL } 300 \\
\text { ASTM } 5174-91 \\
\text { EPA } 353.1 \\
\text { EPA CLP } \\
\text { EPA CLP } \\
\text { EPA CLP } \\
\text { EPA CLP } \\
\text { EPA } 160.2 \\
\text { EPA } 900.0 \\
\text { EPA } 900.0 \\
\text { ASTM } 5174-91 \\
\text { EPA } 353.1 \\
\text { EPA } 160.2 \\
\text { EPA } 353.1 \\
\text { EPA } 160.2 \\
\text { EPA CLP } \\
\text { EPA CLP } \\
\text { EPA CLP } \\
\text { EPA CLP } \\
\text { EPA CLP } \\
\text { EPA CLP } \\
\text { EPA CLP } \\
\text { EPA CLP } \\
\text { EPA CLP } \\
\text { EPA CLP } \\
\text { EPA CLP } \\
\text { EPA CLP } \\
\text { EPA CLP } \\
\text { EPA CLP } \\
\text { EPA CLP } \\
\text { EPA CLP } \\
\text { EPA CLP } \\
\text { EPA CLP } \\
\text { EPA CLP } \\
\text { EPA CLP } \\
\text { EPA CLP } \\
\text { EPA CLP } \\
\text { EPA CLP } \\
\text { EPA } 353.1 \\
\text { EPA CLP } \\
\text { EPA CLP } \\
\text { EPA CLP } \\
\text { EPA CLP } \\
\text { EPA } 160.2 \\
\text { EPA } 900.0 \\
\text { EPA } 900.0 \\
\text { ASTM } 5174-91 \\
\text { EPA } 353.1 \\
\text { EPA } 160.2 \\
\end{array}$ \\
\hline
\end{tabular}




\begin{tabular}{|c|c|c|c|c|c|c|}
\hline WSSRAP_ID & DATE_SAM & PARAMETER & CONC & DL & UNITS & METHOD \\
\hline 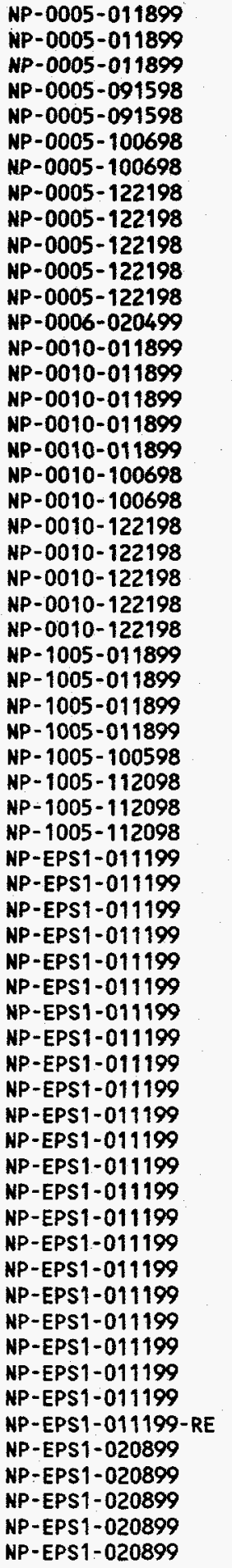 & $\begin{array}{l}01 / 18 / 99 \\
01 / 18 / 99 \\
01 / 18 / 99 \\
09 / 15 / 98 \\
09 / 15 / 98 \\
10 / 06 / 98 \\
10 / 06 / 98 \\
12 / 21 / 98 \\
12 / 21 / 98 \\
12 / 21 / 98 \\
12 / 21 / 98 \\
12 / 21 / 98 \\
02 / 04 / 99 \\
01 / 18 / 99 \\
01 / 18 / 99 \\
01 / 18 / 99 \\
01 / 18 / 99 \\
01 / 18 / 99 \\
10 / 06 / 98 \\
10 / 06 / 98 \\
12 / 21 / 98 \\
12 / 21 / 98 \\
12 / 21 / 98 \\
12 / 21 / 98 \\
12 / 21 / 98 \\
01 / 18 / 99 \\
01 / 18 / 99 \\
01 / 18 / 99 \\
011 / 18 / 99 \\
10 / 05 / 98 \\
11 / 20 / 98 \\
11 / 20 / 98 \\
11 / 20 / 98 \\
01 / 11 / 99 \\
01111 / 99 \\
01 / 11 / 99 \\
01 / 11 / 99 \\
01 / 11 / 99 \\
01111 / 99 \\
01 / 11 / 99 \\
01 / 11 / 99 \\
01 / 11 / 99 \\
01 / 111 / 99 \\
01111 / 99 \\
011 / 11 / 99 \\
01 / 11 / 99 \\
01 / 111 / 99 \\
01 / 111 / 99 \\
01111 / 99 \\
01 / 11 / 99 \\
01 / 11199 \\
01 / 11 / 99 \\
01111 / 99 \\
01111 / 99 \\
01 / 11 / 99 \\
01 / 111 / 99 \\
02 / 08 / 99 \\
02 / 08 / 99 \\
02 / 08 / 99 \\
02 / 08 / 99 \\
02 / 08 / 99\end{array}$ & $\begin{array}{l}\text { GROSS ALPHA } \\
\text { GROSS BETA } \\
\text { URANIUM, TOTAL } \\
\text { NITRATE-N } \\
\text { TOTAL SUSPENDED SOLIDS } \\
\text { NITRATE-N } \\
\text { TOTAL SUSPENDED SOLIOS } \\
\text { NITRATE-N } \\
\text { TOTAL SUSPENDED SOLIDS } \\
\text { GROSS ALPHA } \\
\text { GROSS BETA } \\
\text { URANIU, TOTAL } \\
\text { TOTAL SUSPENDED SOLIDS } \\
\text { NITRATE-N } \\
\text { TOTAL SUSPENDED SOLIDS } \\
\text { GROSS ALPHA } \\
\text { GROSS BETA } \\
\text { URANIUM, TOTAL } \\
\text { NITRATE-N } \\
\text { TOTAL SUSPENDED SOLIDS } \\
\text { NITRATE-N } \\
\text { TOTAL SUSPENDED SOLIDS } \\
\text { GROSS ALPHA } \\
\text { GROSS BETA } \\
\text { URANIUM, TOTAL } \\
\text { TOTAL SUSPENDED SOLIDS } \\
\text { GROSS ALPHA } \\
\text { GROSS BETA } \\
\text { URANIUM, TOTAL } \\
\text { TOTAL SUSPENDED SOLIDS } \\
\text { GROSS ALPHA } \\
\text { GROSS BETA } \\
\text { URANIUM, TOTAL } \\
\text { CHLORIDE } \\
\text { FLUORIDE } \\
\text { NITRATE-N } \\
\text { SULFATE } \\
\text { ARSENIC } \\
\text { CHROMIUM } \\
\text { LEAD } \\
\text { MANGANESE } \\
\text { MERCURY } \\
\text { SELENIUM } \\
\text { CHEMICAL OXYGEN DEMAND } \\
\text { CYANIDE, AMENABLE } \\
\text { TOTAL SUSPENDED SOLIDS } \\
\text { GROSS ALPHA } \\
\text { GROSS BETA } \\
\text { RADIUM-226 } \\
\text { RADIUM-228 } \\
\text { THORIUM-228 } \\
\text { THORIUM-23O } \\
\text { THORIUM-232 } \\
\text { URANIUM, TOTAL } \\
2,4-D I N I T R O T O L U E N E ~ \\
\text { GROSS BETA } \\
\text { CHLORIDE } \\
\text { FLUORIDE } \\
\text { NITRATE-N } \\
\text { SULFATE } \\
\text { ARSENIC }\end{array}$ & 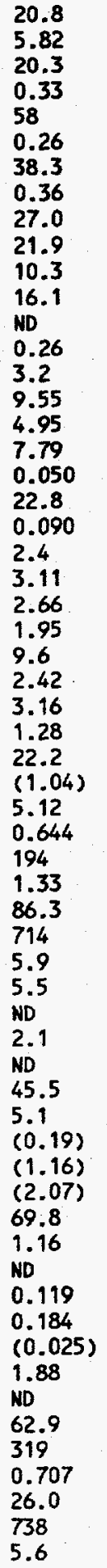 & $\begin{array}{l}0.446 \\
1.05 \\
0.0643 \\
0.020 \\
5.00 \\
0.008 \\
1.01 \\
0.008 \\
1.29 \\
2.15 \\
2.01 \\
0.064 \\
0.00 \\
0.00 \\
1.29 \\
0.47 \\
1.1 \\
0.06 \\
0.003 \\
1.29 \\
0.00 \\
1 \\
1.29 \\
1.62 \\
1.7 \\
0.06 \\
1\end{array}$ & $\begin{array}{l}P C I / L \\
P C I / L \\
P C I / L \\
M G / L \\
M G / L \\
M G / L \\
M G / L \\
M G / L \\
M G / L \\
P C I / L \\
P C I / L \\
P C I / L \\
M G / L \\
M G / L \\
M G / L \\
P C I / L \\
P C I / L \\
P C I / L \\
M G / L \\
M G / L \\
M G / L \\
M G / L \\
P C I / L \\
P C I / L \\
P C I / L \\
M G / L \\
P C I / L \\
P C I / L \\
P C I / L \\
M G / L \\
P C I / L \\
P C I / L \\
P C I / L \\
M G / L \\
M G / L \\
M G / L \\
M G / L \\
U G / L \\
U G / L \\
U G / L \\
U G / L \\
U G / L \\
U G / L \\
M G / L \\
U G / L \\
M G / L \\
P C I / L \\
P C I / L \\
P C I / L \\
P C I / L \\
P C I / L \\
P C I / L \\
P C I / L \\
P C I / L \\
U G / L \\
P C I / L \\
M G / L \\
M G / L \\
M G / L \\
M G / L \\
U G / L \\
\end{array}$ & $\begin{array}{l}\text { EPA } 900.0 \\
\text { EPA } 900.0 \\
\text { ASTM } 5174-91 \\
\text { EPA } 353.1 \\
\text { EPA } 160.2 \\
\text { EPA } 353.1 \\
\text { EPA } 160.2 \\
\text { EPA } 353.1 \\
\text { EPA } 160.2 \\
\text { EPA } 900.0 \\
\text { EPA } 900.0 \\
\text { ASTM } 5174-91 \\
\text { EPA } 160.2 \\
\text { EPA } 353.1 \\
\text { EPA } 160.2 \\
\text { EPA } 900.0 \\
\text { EPA } 900.0 \\
\text { ASTM } 5174-91 \\
\text { EPA } 353.1 \\
\text { EPA } 160.2 \\
\text { EPA } 353.1 \\
\text { EPA } 160.2 \\
\text { EPA } 900.0 \\
\text { EPA } 900.0 \\
\text { ASTM } 5174-91 \\
\text { EPA } 160.2 \\
\text { EPA } 900.0 \\
\text { EPA } 900.0 \\
\text { ASTM } 5174-91 \\
\text { EPA } 160.2 \\
\text { EPA } 900.0 \\
\text { EPA } 900.0 \\
\text { ASTM } 5174-91 \\
\text { EPA } 300.0 \\
\text { EPA } 300.0 \\
\text { EPA } 353.1 \\
\text { EPA } 300.0 \\
\text { EPA } 200.7 \\
\text { EPA } 200.7 \\
\text { EPA } 200.7 \\
\text { EPA } 200.7 \\
\text { EPA } 245.1 \\
\text { EPA } 200.7 \\
\text { EPA } 410.4 \\
\text { EPA } 335.1 \\
\text { EPA } 160.2 \\
\text { EPA } 900.0 \\
\text { EPA } 900.0 \\
\text { SM-705M } \\
\text { EPA } 9320 \\
\text { LANLER200 } \\
\text { LANLER200 } \\
\text { LAMLER200 } \\
\text { ASTM } 5174-91 \\
\text { EPA } 8330 \\
\text { EPA } 900.0 \\
\text { EPA } 300.0 \\
\text { EPA } 300.0 \\
\text { EPA } 353.1 \\
\text { EPA } 300.0 \\
\text { EPA } 200.7 \\
\end{array}$ \\
\hline
\end{tabular}




\begin{tabular}{|c|c|c|c|c|c|c|}
\hline WSSRAP_ID & DATE_SAM & PARAMETER & CONC & DL & UNITS & METHOD \\
\hline 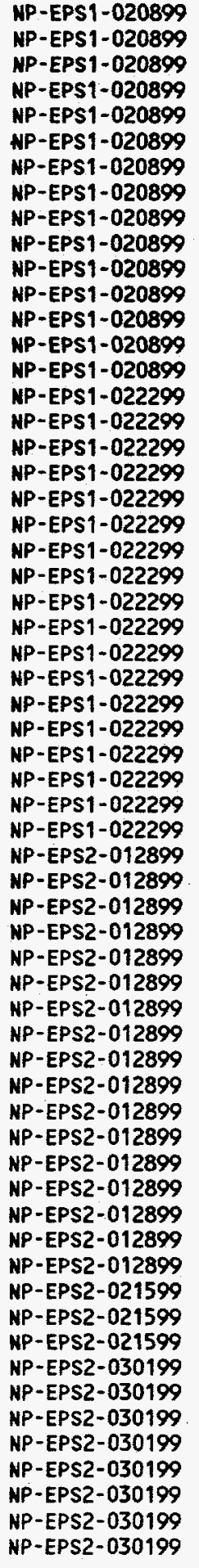 & $\begin{array}{l}02 / 08 / 99 \\
02 / 08 / 99 \\
02 / 08 / 99 \\
02 / 08 / 99 \\
02 / 08 / 99 \\
02 / 08 / 99 \\
02 / 08 / 99 \\
02 / 08 / 99 \\
02 / 08 / 99 \\
02 / 08 / 99 \\
02 / 08 / 99 \\
02 / 08 / 99 \\
02 / 08 / 99 \\
02 / 08 / 99 \\
02 / 08 / 99 \\
02 / 22 / 99 \\
02 / 22 / 99 \\
02 / 22 / 99 \\
02 / 22 / 99 \\
02 / 22 / 99 \\
02 / 22 / 99 \\
02 / 22 / 99 \\
02 / 22 / 99 \\
02 / 22 / 99 \\
02 / 22 / 99 \\
02 / 22 / 99 \\
02 / 22 / 99 \\
02 / 22 / 99 \\
02 / 22 / 99 \\
02 / 22 / 99 \\
02 / 22 / 99 \\
02 / 22 / 99 \\
02 / 22 / 99 \\
01 / 28 / 99 \\
01 / 28 / 99 \\
01 / 28 / 99 \\
01 / 28 / 99 \\
01 / 28 / 99 \\
01 / 28 / 99 \\
01 / 28 / 99 \\
01 / 28 / 99 \\
01 / 28 / 99 \\
01 / 28 / 99 \\
01 / 28 / 99 \\
01 / 28 / 99 \\
01 / 28 / 99 \\
01 / 28 / 99 \\
01 / 28 / 99 \\
01 / 28 / 99 \\
01 / 28 / 99 \\
02 / 15 / 99 \\
02 / 15 / 99 \\
02 / 15 / 99 \\
03 / 01 / 99 \\
03 / 01 / 99 \\
03 / 01 / 99 \\
03 / 01 / 99 \\
03 / 01 / 99 \\
03 / 01 / 99 \\
03 / 01 / 99 \\
03 / 01 / 99\end{array}$ & 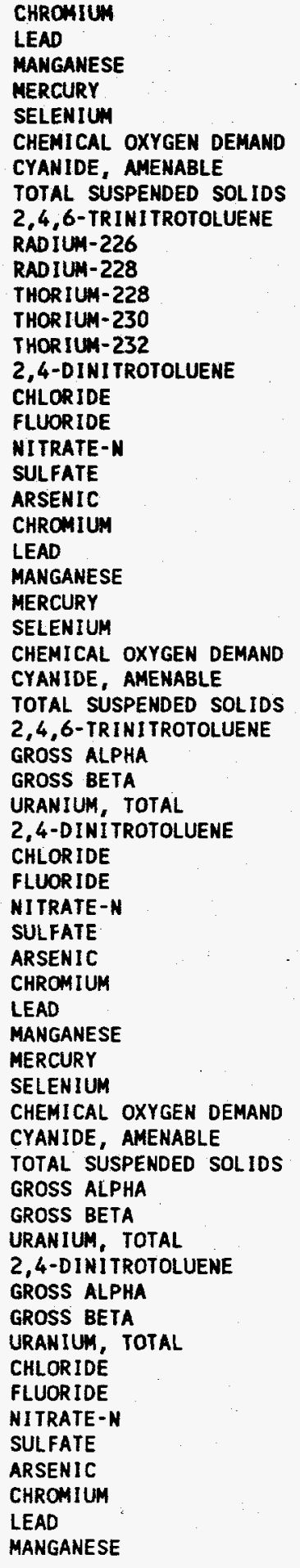 & $\begin{array}{l}4.5 \\
\text { ND } \\
3.4 \\
\text { ND } \\
23.6 \\
\text { ND } \\
5.17 \\
3.02 \\
\text { ND } \\
0.136 \\
\text { ND } \\
(0.061) \\
0.251 \\
0.078 \\
\text { ND } \\
484 \\
0.377 \\
30.0 \\
867 \\
6.5 \\
9.9 \\
2.5 \\
1.7 \\
\text { ND } \\
35.7 \\
(0.567) \\
\text { ND } \\
(1.06) \\
\text { ND } \\
10.4 \\
\text { ND } \\
1.49 \\
\text { ND } \\
268 \\
0.548 \\
36.2 \\
666 \\
4.2 \\
1.7 \\
\text { ND } \\
6.3 \\
\text { ND } \\
30.4 \\
6.1 \\
\text { ND } \\
2.39 \\
11.6 \\
46.9 \\
4.03 \\
\text { ND } \\
(3.5) \\
26.6 \\
1.76 \\
424 \\
\text { ND } \\
49.6 \\
806 \\
\text { ND } \\
4.8 \\
\text { ND } \\
1.3\end{array}$ & 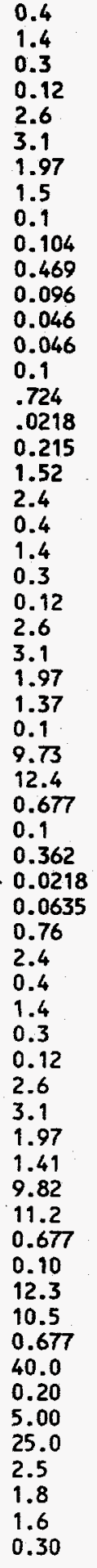 & $\begin{array}{l}U G / L \\
U G / L \\
U G / L \\
U G / L \\
U G / L \\
M G / L \\
U G / L \\
M G / L \\
U G / L \\
P C I / L \\
P C I / L \\
P C I / L \\
P C I / L \\
P C I / L \\
U G / L \\
M G / L \\
M G / L \\
M G / L \\
M G / L \\
U G / L \\
U G / L \\
U G / L \\
U G / L \\
U G / L \\
U G / L \\
M G / L \\
U G / L \\
M G / L \\
U G / L \\
P C I / L \\
P C I / L \\
P C I / L \\
U G / L \\
M G / L \\
M G / L \\
M G / L \\
M G / L \\
U G / L \\
U G / L \\
U G / L \\
U G / L \\
U G / L \\
U G / L \\
M G / L \\
U G / L \\
M G / L \\
P C I / L \\
P C I / L \\
P C I / L \\
U G / L \\
P C I / L \\
P C I / L \\
P C I / L \\
M G / L \\
M G / L \\
M G / L \\
M G / L \\
U G / L \\
U G / L \\
U G / L \\
U G / L \\
\end{array}$ & $\begin{array}{l}\text { EPA } 200.7 \\
\text { EPA } 200.7 \\
\text { EPA } 200.7 \\
\text { EPA } 245.1 \\
\text { EPA } 200.7 \\
\text { EPA } 410.4 \\
\text { EPA } 335.1 \\
\text { EPA } 160.2 \\
\text { EPA } 8330 \\
\text { SM-705M } \\
\text { EPA } 9320 \\
\text { LANL } \\
\text { LAN200 } \\
\text { LANL } \\
\text { ER200 } \\
\text { EPA } 8330 \\
\text { EPA } 300.0 \\
\text { EPA } 300.0 \\
\text { EPA } 353.1 \\
\text { EPA } 300.0 \\
\text { EPA } 200.7 \\
\text { EPA } 200.7 \\
\text { EPA } 200.7 \\
\text { EPA } 200.7 \\
\text { EPA } 245.1 \\
\text { EPA } 200.7 \\
\text { EPA } 410.4 \\
\text { EPA } 335.1 \\
\text { EPA } 160.2 \\
\text { EPA } 8330 \\
\text { EPA } 900.0 \\
\text { EPA } 900.0 \\
\text { ASTM5174M } \\
\text { EPA } 8330 \\
\text { EPA } 300.0 \\
\text { EPA } 300.0 \\
\text { EPA } 300.0 \\
\text { EPA } 300.0 \\
\text { EPA } 200.7 \\
\text { EPA } 200.7 \\
\text { EPA } 200.7 \\
\text { EPA } 200.7 \\
\text { EPA } 245.1 \\
\text { EPA } 200.7 \\
\text { EPA } 410.4 \\
\text { EPA } 335.1 \\
\text { EPA } 160.2 \\
\text { EPA } 900.0 \\
\text { EPA } 900.0 \\
\text { ASTM } 5174-91 \\
\text { EPA } 8330 \\
\text { EPA } 900.0 \\
\text { EPA } 900.0 \\
\text { ASTM } 5174-91 \\
\text { EPA } 300.0 \\
\text { EPA } 300.0 \\
\text { EPA } 353.1 \\
\text { EPA } 300.0 \\
\text { EPA } 200.7 \\
\text { EPA } 200.7 \\
\text { EPA } 200.7 \\
\text { EPA } 200.7 \\
\end{array}$ \\
\hline
\end{tabular}




\begin{tabular}{|c|c|c|c|c|c|c|}
\hline WSSRAP_ID & DATE_SAM & PARAMETER & CONC & DL & UNITS & METHOD \\
\hline 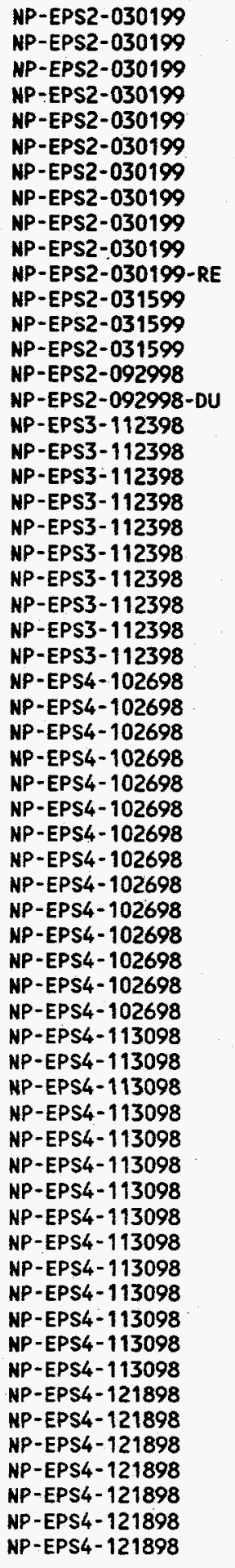 & $\begin{array}{l}03 / 01 / 99 \\
03 / 01 / 99 \\
03 / 01 / 99 \\
03 / 01 / 99 \\
03 / 01 / 99 \\
03 / 01 / 99 \\
03 / 01 / 99 \\
03 / 01 / 99 \\
03 / 01 / 99 \\
03 / 01 / 99 \\
03 / 01 / 99 \\
03 / 15 / 99 \\
03 / 15 / 99 \\
03 / 15 / 99 \\
09 / 29 / 98 \\
09 / 29 / 98 \\
11 / 23 / 98 \\
11 / 23 / 98 \\
11 / 23 / 98 \\
11 / 23 / 98 \\
11 / 23 / 98 \\
11 / 23 / 98 \\
11 / 23 / 98 \\
11 / 23 / 98 \\
11 / 23 / 98 \\
11 / 23 / 98 \\
10 / 26 / 98 \\
10 / 26 / 98 \\
10 / 26 / 98 \\
10 / 26 / 98 \\
10 / 26 / 98 \\
10 / 26 / 98 \\
10 / 26 / 98 \\
10 / 26 / 98 \\
10 / 26 / 98 \\
10 / 26 / 98 \\
10 / 26 / 98 \\
10 / 26 / 98 \\
10 / 26 / 98 \\
10 / 26 / 98 \\
11 / 30 / 98 \\
11 / 30 / 98 \\
11 / 30 / 98 \\
11 / 30 / 98 \\
11 / 30 / 98 \\
11 / 30 / 98 \\
11 / 30 / 98 \\
111 / 30 / 98 \\
11 / 30 / 98 \\
11 / 30 / 98 \\
11 / 30 / 98 \\
11130 / 98 \\
11130 / 98 \\
11 / 30 / 98 \\
12 / 18 / 98 \\
12 / 18 / 98 \\
12 / 18 / 98 \\
12 / 18 / 98 \\
12 / 18 / 98 \\
12 / 18 / 98 \\
12 / 18 / 98\end{array}$ & $\begin{array}{l}\text { MERCURY } \\
\text { SELENIUM } \\
\text { CHEMICAL OXYGEN DEMAND } \\
\text { CYANIDE, AMENABLE } \\
\text { CYANIDE, TOTAL } \\
\text { TOTAL SUSPENDED SOLIDS } \\
2,4-D I N I T R O T O L U E N E \\
\text { GROSS ALPHA } \\
\text { GROSS BETA } \\
\text { URANIUM, TOTAL } \\
\text { SELENIUM } \\
\text { GROSS ALPHA } \\
\text { GROSS BETA } \\
\text { URANIUM, TOTAL } \\
\text { POLONIUM-210 } \\
\text { POLONIUH-210 } \\
2,4-D I N I T R O T O L U E N E \\
\text { GROSS ALPHA } \\
\text { GROSS BETA } \\
\text { RADIUN-226 } \\
\text { RADIUM-228 } \\
\text { RADON-222 } \\
\text { THORIUM-228 } \\
\text { THORIUM-230 } \\
\text { THORIUM-232 } \\
\text { URANIUM, TOTAL } \\
\text { CHLORIDE } \\
\text { FLUORIDE } \\
\text { NITRATE-N } \\
\text { SULFATE } \\
\text { ARSENIC } \\
\text { CHROMIUM } \\
\text { LEAD } \\
\text { MANGANESE } \\
\text { MERCURY } \\
\text { SELENIUM } \\
\text { CHEMICAL OXYGEN DEMAND } \\
\text { CYANIDE, AMENABLE } \\
\text { TOTAL SUSPENDED SOLIDS } \\
2,4-D I N I T R O T O L U E N E \\
\text { CHLORIDE } \\
\text { FLUORIDE } \\
\text { NITRATE-N } \\
\text { SULFATE } \\
\text { ARSENIC } \\
\text { CHROMIUM } \\
\text { LEAD } \\
\text { MANGANESE } \\
\text { MERCURY } \\
\text { SELENIUM } \\
\text { CHEMICAL OXYGEN DEMAND } \\
\text { CYANIDE, AMENABLE } \\
\text { TOTAL SUSPENDED SOLIDS } \\
2,4-D I N I T R O T O L U E N E \\
\text { CHLORIDE } \\
\text { FLUORIDE } \\
\text { NITRATE-N } \\
\text { SULFATE } \\
\text { ARSENIC } \\
\text { CHRONIUM } \\
\text { LEAD }\end{array}$ & 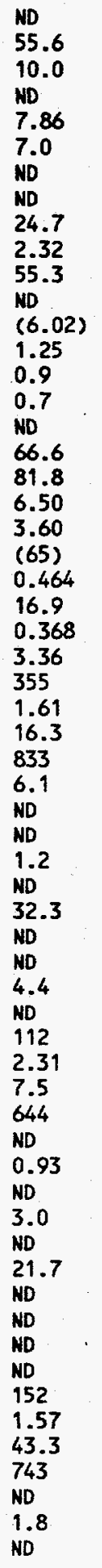 & 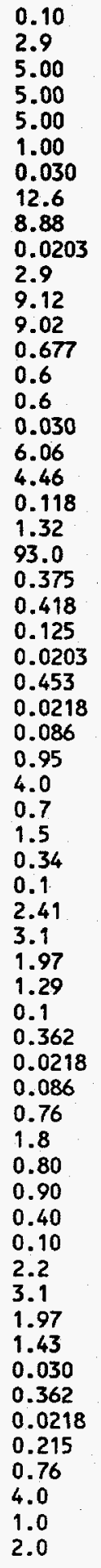 & $\begin{array}{l}U G / L \\
U G / L \\
M G / L \\
U G / L \\
U G / L \\
M G / L \\
U G / L \\
P C I / L \\
P C I / L \\
P C I / L \\
U G / L \\
P C I / L \\
P C I / L \\
P C I / L \\
P C I / L \\
P C I / L \\
U G / L \\
P C I / L \\
P C I / L \\
P C I / L \\
P C I / L \\
P C I / L \\
P C I / L \\
P C I / L \\
P C I / L \\
P C I / L \\
M G / L \\
M G / L \\
M G / L \\
M G / L \\
U G / L \\
U G / L \\
U G / L \\
U G / L \\
U G / L \\
U G / L \\
M G / L \\
U G / L \\
M G / L \\
U G / L \\
M G / L \\
M G / L \\
M G / L \\
M G / L \\
U G / L \\
U G / L \\
U G / L \\
U G / L \\
U G / L \\
U G / L \\
M G / L \\
U G / L \\
M G / L \\
U G / L \\
M G / L \\
M G / L \\
M G / L \\
M G / L \\
U G / L \\
U G / L \\
U G / L \\
\end{array}$ & $\begin{array}{l}\text { EPA } 245.1 \\
\text { EPA } 200.7 \\
\text { EPA } 410.4 \\
\text { EPA } 335.1 \\
\text { EPA } 335.2 \\
\text { EPA } 160.2 \\
\text { USATHAMA } \\
\text { EPA } 900.0 \\
\text { EPA } 900.0 \\
\text { ASTM } 5174-91 \\
\text { EPA } 200.7 \\
\text { EPA } 900.0 \\
\text { EPA } 900.0 \\
\text { ASTM5174M } \\
\text { ALPHA SPEC } \\
\text { ALPHA SPEC } \\
\text { USATHAMA } \\
\text { EPA } 900.0 \\
\text { EPA } 900.0 \\
\text { EPA } 903.0 \\
\text { EPA } 904.0 \\
\text { EPA } \\
\text { EML TH-01 } \\
\text { EML TH-01 } \\
\text { EML TH-01 } \\
\text { ASTM } 5174-91 \\
\text { EPA } 300.0 \\
\text { EPA } 300.0 \\
\text { EPA } 353.1 \\
\text { EPA } 300.0 \\
\text { EPA } 200.7 \\
\text { EPA } 200.7 \\
\text { EPA } 200.7 \\
\text { EPA } 200.7 \\
\text { EPA } 245.1 \\
\text { EPA } 200.7 \\
\text { EPA } 410.4 \\
\text { EPA } 335.1 \\
\text { EPA } 160.2 \\
\text { EPA } 8330 \\
\text { EPA } 300.0 \\
\text { EPA } 300.0 \\
\text { EPA } 353.1 \\
\text { EPA } 300.0 \\
\text { EPA } 200.7 \\
\text { EPA } 200.7 \\
\text { EPA } 200.7 \\
\text { EPA } 200.7 \\
\text { EPA } 245.1 \\
\text { EPA } 200.7 \\
\text { EPA } 410.4 \\
\text { EPA } 335.1 \\
\text { EPA } 160.2 \\
\text { USATHAMA } \\
\text { EPA } 300.0 \\
\text { EPA } 300.0 \\
\text { EPA } 353.1 \\
\text { EPA } 300.0 \\
\text { EPA } 200 \\
\text { EPA } 200 \\
\text { EPA } 200 \\
\end{array}$ \\
\hline
\end{tabular}




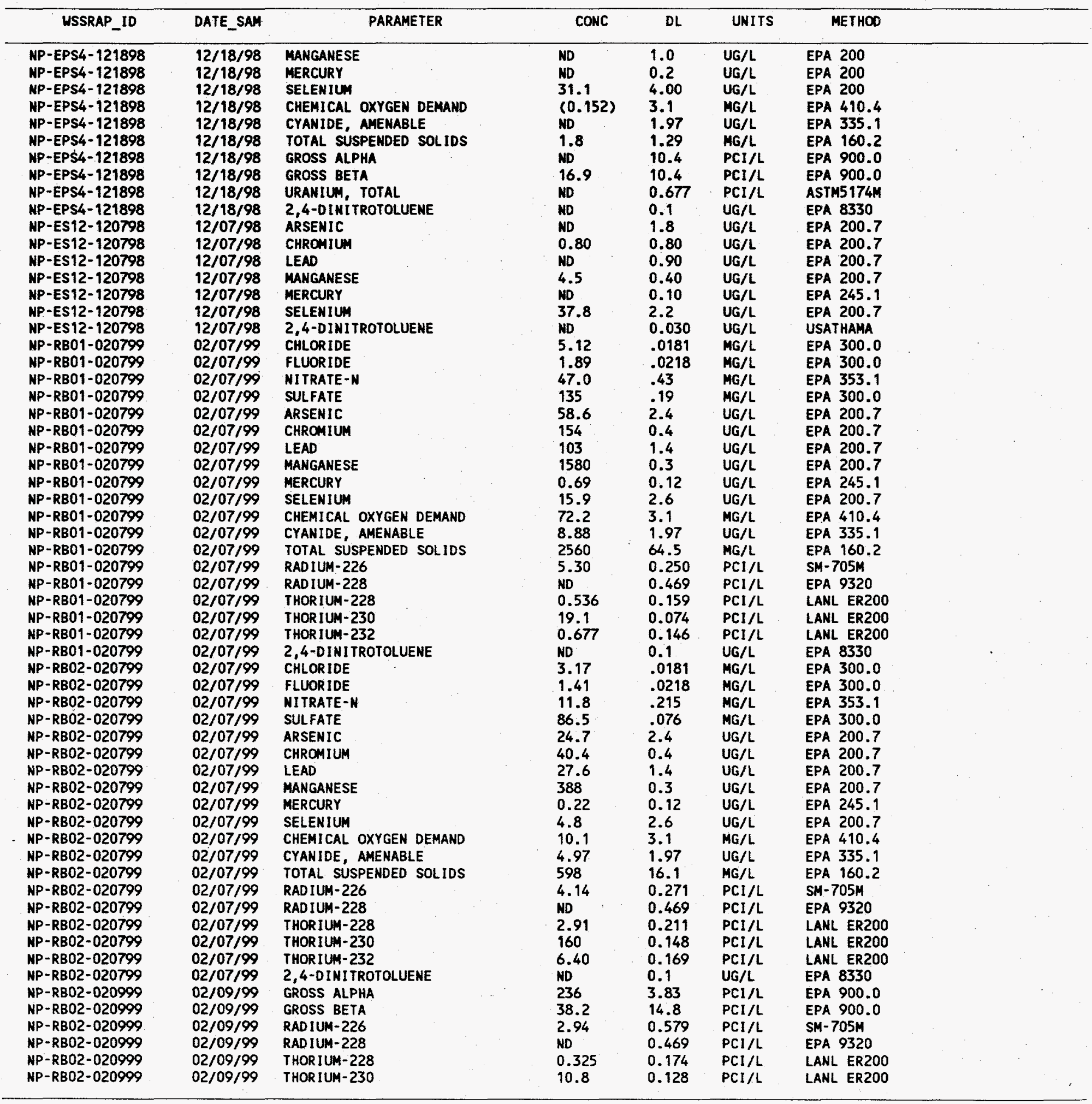




\begin{tabular}{|c|c|c|c|c|c|c|}
\hline MSSRAP_ID & DATE_SAM & PARAMETER & CONC & DL & UNITS & METHOD \\
\hline $\begin{array}{l}\text { NP-RBO2-020999 } \\
\text { NP-RBO2-020999 }\end{array}$ & $\begin{array}{l}02 / 09 / 99 \\
02 / 09 / 99\end{array}$ & $\begin{array}{l}\text { THORIUM-232 } \\
\text { URANIUM, TOTAL }\end{array}$ & $\begin{array}{l}0.623 \\
290\end{array}$ & $\begin{array}{l}0.141 \\
0.677\end{array}$ & $\begin{array}{l}\mathrm{PCI} / \mathrm{L} \\
\mathrm{PCI} / \mathrm{L}\end{array}$ & $\begin{array}{l}\text { LANL ER200 } \\
\text { ASTM } 5174-91\end{array}$ \\
\hline
\end{tabular}


GROUNDWATER 


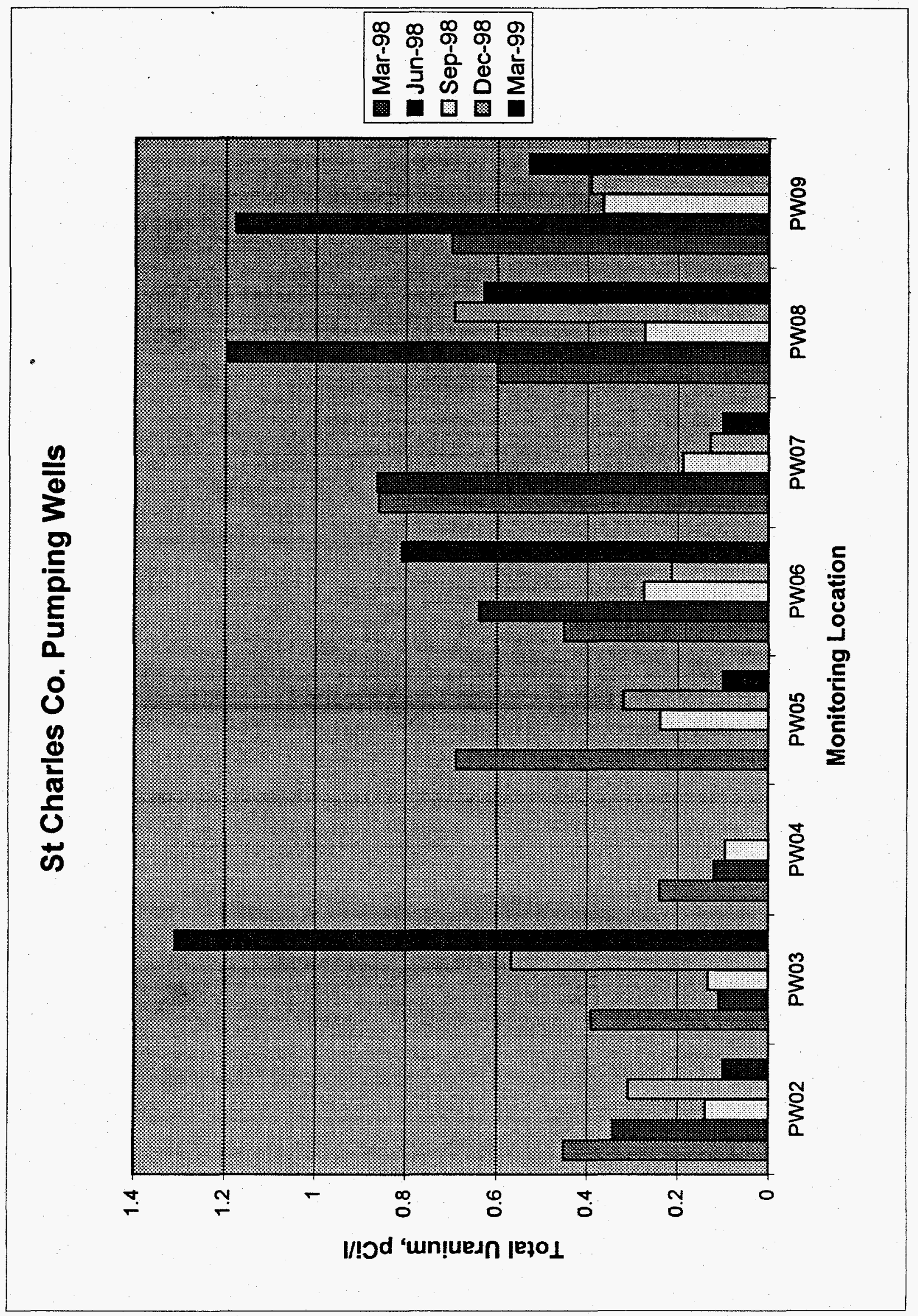




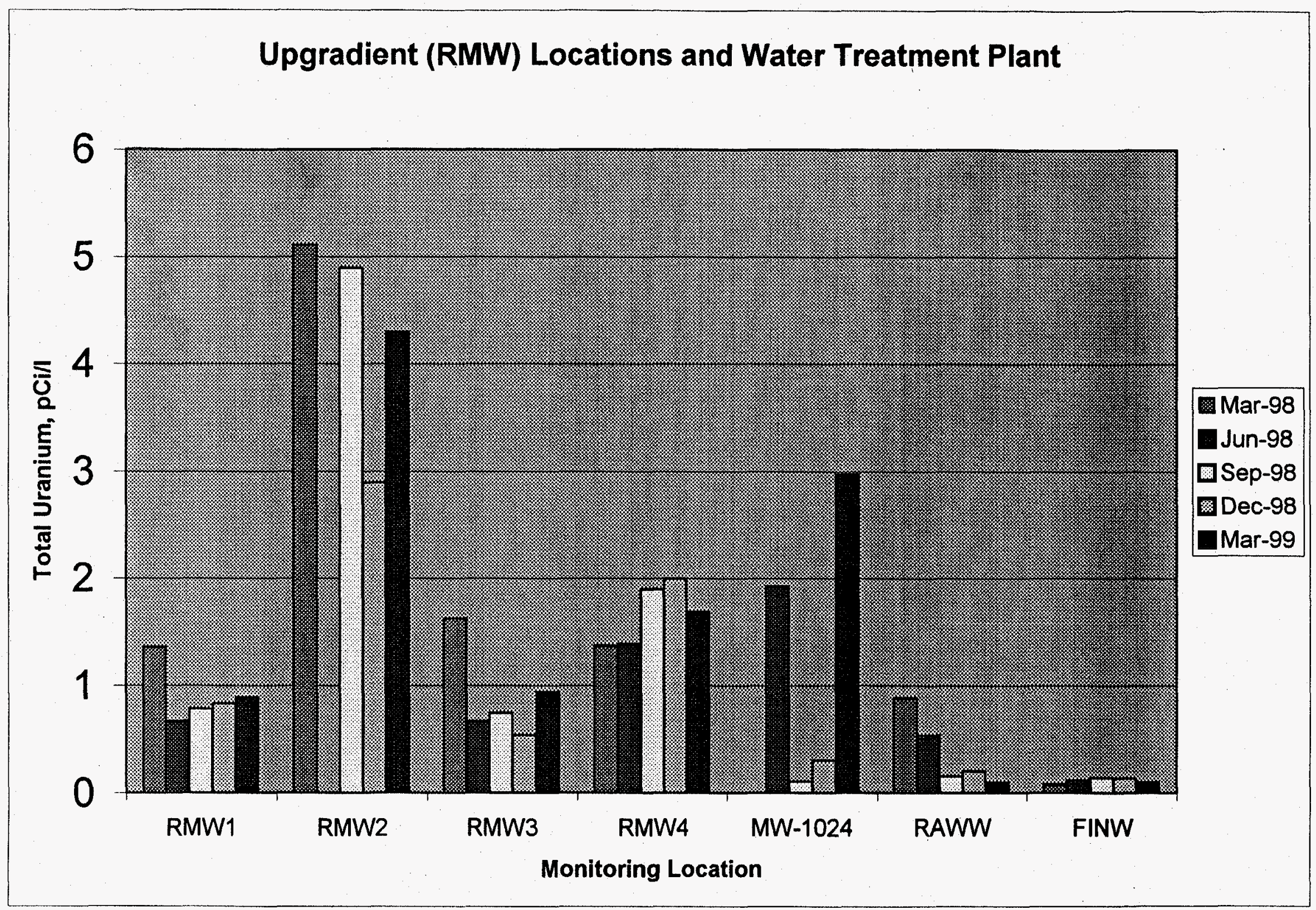


GROUNDWATER

DATA MERGED DURING FIRST QUARTER, 1999

\begin{tabular}{|c|c|c|c|c|c|c|}
\hline WSSRAP_ID & DATE_SAM & PARAMETER & CONC & $D L$ & UNITS & METHOD \\
\hline $\begin{array}{l}G W-1002-0498 \\
G W-1002-0498 \\
G W-1002-0498 \\
G W-1002-0498 \\
G W-1002-0498 \\
G W-1002-0498 \\
G W-1002-0498 \\
G W-1002-0498 \\
G W-1004-0498 \\
G W-1004-0498 \\
G W-1004-0498 \\
G W-1004-0498 \\
G W-1004-0498 \\
G W-1004-0498 \\
G W-1004-0498 \\
G W-1004-0498 \\
G W-1005-0498 \\
G W-1005-0498 \\
G W-1012-100198 \\
G W-1012-100198 \\
G W-1015-0498 \\
G W-1015-0498 \\
G W-1015-0498 \\
G W-1015-0498 \\
G W-1015-0498 \\
G W-1015-0498 \\
G W-1015-0498 \\
G W-1015-0498 \\
G W-1015-0498 \\
G W-1015-0498 \\
G W-1015-0498 \\
G W-1015-0498 \\
G W-1016-0498 \\
G W-1016-0498 \\
G W-1016-0498 \\
G W-1016-0498 \\
G W-1016-0498 \\
G W-1016-0498 \\
G H-1016-0498 \\
G W-1016-0498 \\
G W-1016-0498 \\
G W-1016-0498 \\
G W-1016-0498 \\
G W-1016-0498 \\
G W-1017-0498 \\
G W-1017-0498 \\
G W-1017-0498 \\
G W-1017-0498 \\
G W-1017-0498 \\
G W-1017-0498 \\
G W-1018-0498 \\
G W-1018-0498 \\
G W-1018-0498 \\
G W-1018-0498 \\
G W-1018-0498 \\
G W-1018-0498 \\
G W-1019-0498 \\
G W-1019-0498\end{array}$ & $\begin{array}{l}10 / 08 / 98 \\
10 / 08 / 98 \\
10 / 08 / 98 \\
10 / 08 / 98 \\
10 / 08 / 98 \\
10 / 08 / 98 \\
10 / 08 / 98 \\
10 / 08 / 98 \\
10 / 08 / 98 \\
10 / 08 / 98 \\
10 / 08 / 98 \\
10 / 08 / 98 \\
10 / 08 / 98 \\
10 / 08 / 98 \\
10 / 08 / 98 \\
10 / 08 / 98 \\
10 / 08 / 98 \\
10 / 08 / 98 \\
10 / 01 / 98 \\
10 / 01 / 98 \\
10 / 22 / 98 \\
10 / 22 / 98 \\
10 / 22 / 98 \\
10 / 22 / 98 \\
10 / 22 / 98 \\
10 / 22 / 98 \\
10 / 22 / 98 \\
10 / 22 / 98 \\
10 / 22 / 98 \\
10 / 22 / 98 \\
10 / 22 / 98 \\
10 / 22 / 98 \\
10 / 22 / 98 \\
10 / 22 / 98 \\
10 / 22 / 98 \\
10 / 22 / 98 \\
10 / 22 / 98 \\
10 / 22 / 98 \\
10 / 22 / 98 \\
10 / 22 / 98 \\
10 / 22 / 98 \\
10 / 22 / 98 \\
10 / 22 / 98 \\
10 / 22 / 98 \\
12 / 03 / 98 \\
12 / 03 / 98 \\
12 / 03 / 98 \\
12 / 03 / 98 \\
12 / 03 / 98 \\
12 / 03 / 98 \\
12 / 03 / 98 \\
12 / 03 / 98 \\
12 / 03 / 98 \\
12 / 03 / 98 \\
12 / 03 / 98 \\
12 / 03 / 98 \\
12 / 02 / 98 \\
12 / 02 / 98\end{array}$ & $\begin{array}{l}\text { SULFATE } \\
\text { ANTIMONY } \\
\text { THALLIUM } \\
\text { RADIUH-226 } \\
\text { RADIUM-228 } \\
\text { THORIUM-228 } \\
\text { THORIUM-230 } \\
\text { THORIUH-232 } \\
\text { SULFATE } \\
\text { ANTIMONY } \\
\text { THALLIUM } \\
\text { RADIUN-226 } \\
\text { RADIUM-228 } \\
\text { THORIUH-228 } \\
\text { THORIUM-230 } \\
\text { THORIUM-232 } \\
\text { ANTIMONY } \\
\text { THALLIUM } \\
\text { ANTIMONY } \\
\text { THALLIUM } \\
\text { SULFATE } \\
\text { 1,3,5-TRINITROBENZENE } \\
\text { 1,3-DINITROBENZENE } \\
2,4,6-T R I N I T R O T O L U E N E \\
2,4-D I N I T R O T O L U E N E \\
2,6-D I N I T R O T O L U E N E \\
\text { NITROBENZENE } \\
\text { RADIUM-226 } \\
\text { RADIUM-228 } \\
\text { THORIUM-228 } \\
\text { THORIUM-230 } \\
\text { THORIUM-232 } \\
\text { SULFATE } \\
1,3,5-T R I N I T R O B E N Z E N E \\
1,3-D I H I T R O B E N Z E N E \\
2,4,6-T R I N I T R O T O L U E N E \\
2,4-D I N I T R O T O L U E N E \\
2,6-D I N I T R O T O L U E N E \\
\text { NITROBENZENE } \\
\text { RADIUM-226 } \\
\text { RADIUM-228 } \\
\text { THORIUM-228 } \\
\text { THORIUM-230 } \\
\text { THORIUM-232 } \\
\text { SULFATE } \\
\text { RADIUM-226 } \\
\text { RADIUM-228 } \\
\text { THORIUM-228 } \\
\text { THORIUM-230 } \\
\text { THORIUM-232 } \\
\text { SULFATE } \\
\text { RADIUM-226 } \\
\text { RADIUM-228 } \\
\text { THORIUM-228 } \\
\text { THORIUM-230 } \\
\text { THORIUM-232 } \\
\text { SULFATE } \\
1,3,5-T R I N I T R O B E N Z E N E\end{array}$ & 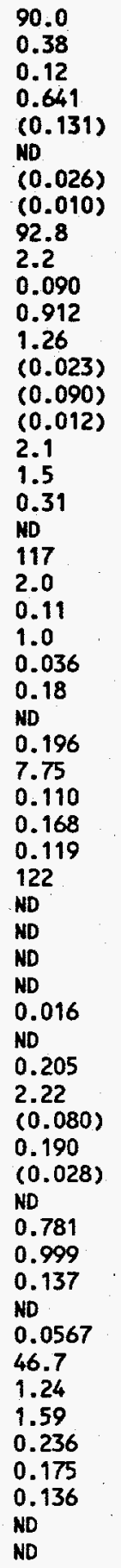 & $\begin{array}{l}0.076 \\
0.05 \\
0.05 \\
0.294 \\
0.04 \\
0.138 \\
0.098 \\
0.068 \\
0.076 \\
0.05 \\
0.05 \\
0.421 \\
0.716 \\
0.136 \\
0.093 \\
0.0876 \\
0.05 \\
0.05 \\
0.05 \\
0.05 \\
0.00 \\
0.00 \\
0.150 \\
0.09 \\
0.150 \\
0.030 \\
0\end{array}$ & $\begin{array}{l}M G / L \\
U G / L \\
U G / L \\
P C I / L \\
P C I / L \\
P C I / L \\
P C I / L \\
P C I / L \\
\text { MG/L } \\
U G / L \\
U G / L \\
P C I / L \\
P C I / L \\
P C I / L \\
P C I / L \\
P C I / L \\
U G / L \\
U G / L \\
U G / L \\
U G / L \\
M G / L \\
U G / L \\
U G / L \\
U G / L \\
U G / L \\
U G / L \\
U G / L \\
P C I / L \\
P C I / L \\
P C I / L \\
P C I / L \\
P C I / L \\
M G / L \\
U G / L \\
U G / L \\
U G / L \\
U G / L \\
U G / L \\
U G / L \\
P C I / L \\
P C I / L \\
P C I / L \\
P C I / L \\
P C I / L \\
M G / L \\
P C I / L \\
P C I / L \\
P C I / L \\
P C I / L \\
P C I / L \\
M G / L \\
P C I / L \\
P C I / L \\
P C I / L \\
P C I / L \\
P C I / L \\
M G / L \\
U G / L \\
\text { U }\end{array}$ & $\begin{array}{l}\text { EPA } 300.0 \\
\text { EPA } 6020 \\
\text { EPA } 6020 \\
\text { EPA } 903.1 \\
\text { EPA } 904.0 \\
\text { HASL } 300 \\
\text { HASL } 300 \\
\text { HASL } 300 \\
\text { EPA } 300.0 \\
\text { EPA } 6020 \\
\text { EPA } 6020 \\
\text { EPA } 903.1 \\
\text { EPA } 904.0 \\
\text { HASL } 300 \\
\text { HASL } 300 \\
\text { HASL } 300 \\
\text { EPA } 6020 \\
\text { EPA } 6020 \\
\text { EPA } 6020 \\
\text { EPA } 6020 \\
\text { EPA } 300.0 \\
\text { USATHAMA } \\
\text { USATHAMA } \\
\text { USATHAMA } \\
\text { USATHAMA } \\
\text { USATHAMA } \\
\text { USATHAMA } \\
\text { EPA } 904.0 \\
\text { EPA } 904.0 \\
\text { NAS-NS-3004 } \\
\text { NAS-NS-3004 } \\
\text { NAS-NS-3004 } \\
\text { EPA } 300.0 \\
\text { USATHAMA } \\
\text { USATHAMA } \\
\text { USATHAMA } \\
\text { USATHAMA } \\
\text { USATHAMA } \\
\text { USATHAMA } \\
\text { EPA } 904.0 \\
\text { EPA } 904.0 \\
\text { NAS-NS-3004 } \\
\text { NAS-NS-3004 } \\
\text { NAS-NS-3004 } \\
\text { EPA } 300.0 \\
\text { EPA } 903.1 \\
\text { EPA } 904.0 \\
\text { HASL } 300 \\
\text { HASL } 300 \\
\text { HASL } 300 \\
\text { EPA } 300.0 \\
\text { EPA } 903.1 \\
\text { EPA } 904.0 \\
\text { HASL } 300 \\
\text { HASL } 300 \\
\text { HASL } 300 \\
\text { EPA } 300.0 \\
\text { USATHAMA }\end{array}$ \\
\hline
\end{tabular}




\begin{tabular}{|c|c|c|c|c|c|c|}
\hline USSRAP_ID & DATE_SAM & PARAMETER & CONC & DL & UNITS & METHOD \\
\hline $\begin{array}{l}\text { GW-1019-0498 } \\
G W-1019-0498 \\
G W-1019-0498 \\
G W-1019-0498 \\
G W-1019-0498 \\
G W-1019-0498 \\
G W-1019-0498 \\
G W-1019-0498 \\
G W-1019-0498 \\
G W-1019-0498 \\
G W-1020-0498 \\
G W-1020-0498 \\
G W-1020-0498 \\
G W-1020-0498 \\
G W-1020-0498 \\
G W-1020-0498 \\
G W-1020-0498 \\
G W-1020-0498 \\
G H-1020-0498 \\
G W-1020-0498 \\
G W-1020-0498 \\
G W-1020-0498 \\
G W-1021-0498 \\
G W-1021-0498 \\
G W-1021-0498 \\
G W-1021-0498 \\
G W-1021-0498 \\
G W-1021-0498 \\
G W-1021-0498 \\
G W-1021-0498 \\
G W-1021-0498 \\
G W-1021-0498 \\
G W-1021-0498 \\
G W-1021-0498 \\
G W-1022-0498 \\
G W-1022-0498 \\
G W-1022-0498 \\
G W-1022-0498 \\
G W-1022-0498 \\
G W-1022-0498 \\
G W-1022-0498 \\
G W-1022-0498 \\
G W-1022-0498 \\
G W-1022-0498 \\
G W-1022-0498 \\
G W-1022-0498 \\
G W-1023-0498 \\
G W-1023-0498 \\
G W-1023-0498 \\
G W-1023-0498 \\
G W-1023-0498 \\
G W-1023-0498 \\
G W-1024-0498 \\
G W-1024-0498 \\
G W-1024-0498 \\
G W-1024-0498 \\
G W-1024-0498 \\
G W-1024-049498 \\
G W-1024-0498 \\
G W-1024-0498\end{array}$ & $\begin{array}{l}12 / 02 / 98 \\
12 / 02 / 98 \\
12 / 02 / 98 \\
12 / 02 / 98 \\
12 / 02 / 98 \\
12 / 02 / 98 \\
12 / 02 / 98 \\
12 / 02 / 98 \\
12 / 02 / 98 \\
12 / 02 / 98 \\
12 / 02 / 98 \\
12 / 02 / 98 \\
12 / 02 / 98 \\
12 / 02 / 98 \\
12 / 02 / 98 \\
12 / 02 / 98 \\
12 / 02 / 98 \\
12 / 02 / 98 \\
12 / 02 / 98 \\
12 / 02 / 98 \\
12 / 02 / 98 \\
12 / 02 / 98 \\
12 / 02 / 98 \\
12 / 02 / 98 \\
12 / 02 / 98 \\
12 / 02 / 98 \\
12 / 02 / 98 \\
12 / 02 / 98 \\
12 / 02 / 98 \\
12 / 02 / 98 \\
12 / 02 / 98 \\
12 / 02 / 98 \\
12 / 02 / 98 \\
12 / 02 / 98 \\
12 / 02 / 98 \\
12 / 02 / 98 \\
12 / 02 / 98 \\
12 / 02 / 98 \\
12 / 02 / 98 \\
12 / 02 / 98 \\
12 / 02 / 98 \\
12 / 02 / 98 \\
12 / 02 / 98 \\
12 / 02 / 98 \\
12 / 02 / 98 \\
12 / 02 / 98 \\
12 / 03 / 98 \\
12 / 03 / 98 \\
12 / 03 / 98 \\
12 / 03 / 98 \\
12 / 03 / 98 \\
12 / 03 / 98 \\
12 / 14 / 98 \\
12 / 14 / 98 \\
12 / 14 / 98 \\
12 / 14 / 98 \\
12 / 14 / 98 \\
12 / 14 / 98 \\
12 / 14 / 98 \\
12 / 14 / 98 \\
12 / 14 / 98\end{array}$ & 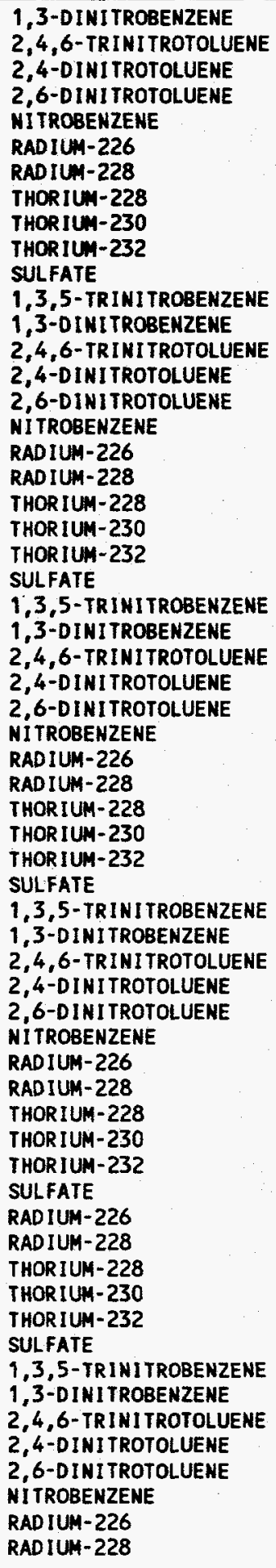 & $\begin{array}{l}\text { ND } \\
\text { ND } \\
\text { ND } \\
\text { ND } \\
\text { ND } \\
1.94 \\
3.01 \\
0.228 \\
0.229 \\
0.153 \\
1.03 \\
\text { ND } \\
\text { ND } \\
\text { ND } \\
\text { ND } \\
\text { ND } \\
\text { ND } \\
0.903 \\
1.48 \\
\text { (0.084) } \\
\text { (0.038) } \\
0.0419 \\
0.103 \\
\text { ND } \\
\text { ND } \\
\text { ND } \\
\text { ND } \\
\text { ND } \\
\text { ND } \\
2.08 \\
3.58 \\
0.276 \\
0.161 \\
0.0927 \\
\text { ND } \\
\text { ND } \\
\text { ND } \\
\text { ND } \\
\text { ND } \\
\text { ND } \\
\text { ND } \\
0.479 \\
\text { ND } \\
0.1 \\
0.0827 \\
0.063 \\
2.98 \\
3.02 \\
3.77 \\
0.284 \\
0.273 \\
0.257 \\
3.5 \\
\text { ND } \\
\text { ND } \\
\text { ND } \\
\text { ND } \\
\text { ND } \\
\text { ND } \\
0.432 \\
0.823\end{array}$ & $\begin{array}{l}0.090 \\
0.030 \\
0.030 \\
0.010 \\
0.030 \\
0.374 \\
0.997 \\
0.123 \\
0.0785 \\
0.069 \\
0\end{array}$ & 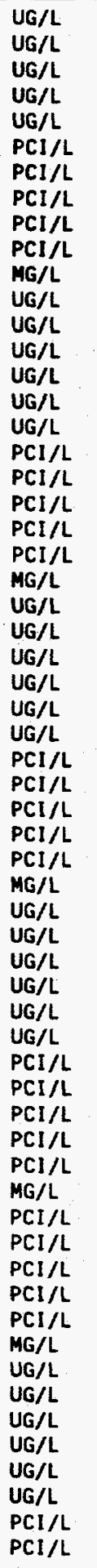 & $\begin{array}{l}\text { USATHAMA } \\
\text { USATHAMA } \\
\text { USATHAMA } \\
\text { USATHAMA } \\
\text { USATHAMA } \\
\text { EPA } 903.1 \\
\text { EPA } 904.0 \\
\text { HASL } 300 \\
\text { HASL } 300 \\
\text { HASL } 300 \\
\text { EPA } 300.0 \\
\text { USATHAMA } \\
\text { USATHAMA } \\
\text { USATHAMA } \\
\text { USATHAMA } \\
\text { USATHAMA } \\
\text { USATHAMA } \\
\text { EPA } 903.1 \\
\text { EPA } 904.0 \\
\text { HASL } 300 \\
\text { HASL } 300 \\
\text { HASL } 300 \\
\text { EPA } 300.0 \\
\text { USATHAMA } \\
\text { USATHAMA } \\
\text { USATHAMA } \\
\text { USATHAMA } \\
\text { USATHAMA } \\
\text { USATHAMA } \\
\text { EPA } 903.1 \\
\text { EPA } 904.0 \\
\text { HASL } 300 \\
\text { HASL } 300 \\
\text { HASL } 300 \\
\text { EPA } 300.0 \\
\text { USATHAMA } \\
\text { USATHAMA } \\
\text { USATHAMA } \\
\text { USATHAMA } \\
\text { USATHAMA } \\
\text { USATHAMA } \\
\text { EPA } 903.1 \\
\text { EPA } 904.0 \\
\text { HASL } 300 \\
\text { HASL } 300 \\
\text { HASL } 300 \\
\text { EPA } 300.0 \\
\text { EPA } 903.1 \\
\text { EPA } 904.0 \\
\text { HASL } 300 \\
\text { HASL } 300 \\
\text { HASL } 300 \\
\text { EPA } 300.0 \\
\text { USATHAMA } \\
\text { USATHAMA } \\
\text { USATHAMA } \\
\text { USATHAMA } \\
\text { USATHAMA } \\
\text { USATHAMA } \\
\text { EPA } 903.1 \\
\text { EPA } 904.0\end{array}$ \\
\hline
\end{tabular}




\begin{tabular}{|c|c|c|c|c|c|c|}
\hline WSSRAP_ID & DATE_SAM & PARAMETER & CONC & DL & UNITS & METHOD \\
\hline 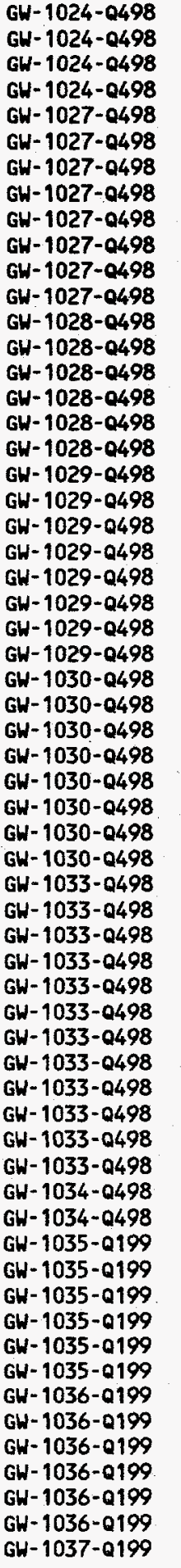 & $\begin{array}{l}12 / 14 / 98 \\
12 / 14 / 98 \\
12 / 14 / 98 \\
12 / 14 / 98 \\
10 / 07 / 98 \\
10 / 07 / 98 \\
10 / 07 / 98 \\
10 / 07 / 98 \\
10 / 07 / 98 \\
10 / 07 / 98 \\
10 / 07 / 98 \\
10 / 07 / 98 \\
10 / 20 / 98 \\
10 / 20 / 98 \\
10 / 20 / 98 \\
10 / 20 / 98 \\
10 / 20 / 98 \\
10 / 20 / 98 \\
10 / 08 / 98 \\
10 / 08 / 98 \\
10 / 08 / 98 \\
10 / 08 / 98 \\
10 / 08 / 98 \\
10 / 08 / 98 \\
10 / 08 / 98 \\
10 / 08 / 98 \\
10 / 08 / 98 \\
10 / 08 / 98 \\
10 / 08 / 98 \\
10 / 08 / 98 \\
10 / 08 / 98 \\
10 / 08 / 98 \\
10 / 08 / 98 \\
10 / 08 / 98 \\
12 / 02 / 98 \\
12 / 02 / 98 \\
12 / 02 / 98 \\
12 / 02 / 98 \\
12 / 02 / 98 \\
12 / 02 / 98 \\
12 / 02 / 98 \\
12 / 02 / 98 \\
12 / 02 / 98 \\
12 / 02 / 98 \\
12 / 02 / 98 \\
12 / 02 / 98 \\
10 / 01 / 98 \\
10 / 01 / 98 \\
01 / 15 / 99 \\
01 / 15 / 99 \\
01 / 15 / 99 \\
01 / 15 / 99 \\
01 / 15 / 99 \\
01 / 15 / 99 \\
01 / 19 / 99 \\
01 / 19 / 99 \\
01 / 19 / 99 \\
01 / 19 / 99 \\
01 / 19 / 99 \\
01 / 19 / 99 \\
01 / 19 / 99\end{array}$ & 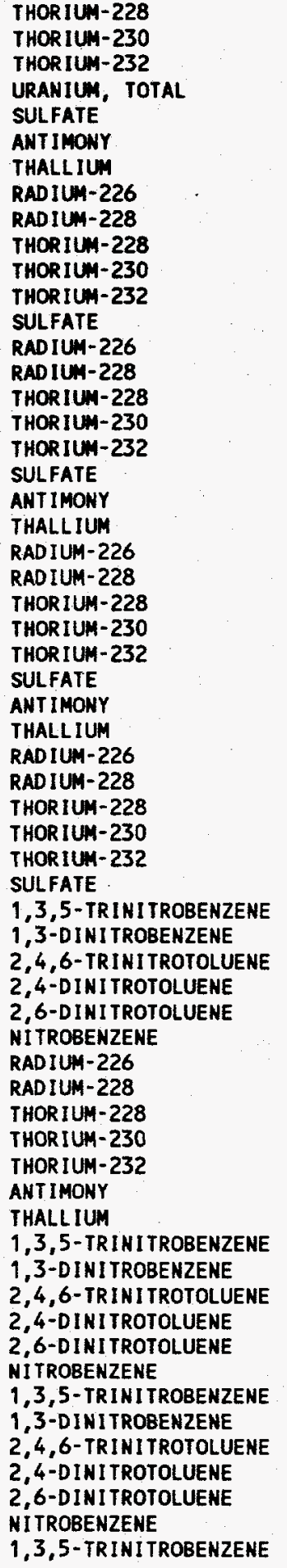 & $\begin{array}{l}0.0858 \\
0.0605 \\
(0.0110) \\
0.161 \\
92.8 \\
\text { ND } \\
\text { ND } \\
(0.286) \\
(0.019) \\
(0.015) \\
0.112 \\
(0.026) \\
60.7 \\
0.492 \\
1.33 \\
\text { ND } \\
(0.047) \\
(0.031) \\
109 \\
0.44 \\
\text { ND } \\
0.551 \\
(0.550) \\
(0.007) \\
(0.041) \\
\text { ND } \\
\text { T5.0 } \\
0.3 \\
0.080 \\
0.576 \\
(0.052) \\
0.205 \\
(0.039) \\
0.165 \\
1.66 \\
\text { ND } \\
\text { ND } \\
\text { ND } \\
\text { ND } \\
\text { ND } \\
\text { ND } \\
1.34 \\
\text { ND } \\
\text { ND } \\
(0.027) \\
(0.004) \\
\text { ND } \\
\text { ND } \\
\text { ND } \\
\text { ND } \\
\text { ND } \\
\text { ND } \\
\text { ND } \\
\text { ND } \\
\text { ND } \\
\text { ND } \\
\text { ND } \\
\text { ND } \\
\text { ND } \\
\text { ND } \\
\text { ND }\end{array}$ & $\begin{array}{l}0.0832 \\
0.0523 \\
0.0418 \\
0.064 \\
0.076 \\
0.05 \\
0.05 \\
0.346 \\
0.083 \\
0.145 \\
0.06 \\
0\end{array}$ & $\begin{array}{l}P C I / L \\
P C I / L \\
P C I / L \\
P C I / L \\
M G / L \\
U G / L \\
U G / L \\
P C I / L \\
P C I / L \\
P C I / L \\
P C I / L \\
P C I / L \\
M G / L \\
P C I / L \\
P C I / L \\
P C I / L \\
P C I / L \\
P C I / L \\
M G / L \\
U G / L \\
U G / L \\
P C I / L \\
P C I / L \\
P C I / L \\
P C I / L \\
P C I / L \\
M G / L \\
U G / L \\
U G / L \\
P C I / L \\
P C I / L \\
P C I / L \\
P C I / L \\
P C I / L \\
M G / L \\
U G / L \\
U G / L \\
U G / L \\
U G / L \\
U G / L \\
U G / L \\
P C I / L \\
P C I / L \\
P C I / L \\
P C I / L \\
P C I / L \\
U G / L \\
U G / L \\
U G / L \\
U G / L \\
U G / L \\
U G / L \\
U G / L \\
U G / L \\
U G / L \\
U G / L \\
U G / L \\
U G / L \\
U G / L \\
U G / L \\
U G / L \\
\end{array}$ & $\begin{array}{l}\text { HASL } 300 \\
\text { HASL } 300 \\
\text { HASL } 300 \\
\text { ASTM } 5174-91 \\
\text { EPA } 300.0 \\
\text { EPA } 6020 \\
\text { EPA } 6020 \\
\text { EPA } 903.1 \\
\text { EPA } 904.0 \\
\text { HASL } 300 \\
\text { HASL } 300 \\
\text { HASL } 300 \\
\text { EPA } 300.0 \\
\text { EPA } 904.0 \\
\text { EPA } 904.0 \\
\text { NAS-NS-3004 } \\
\text { NAS-NS-3004 } \\
\text { NAS-NS-3004 } \\
\text { EPA } 300.0 \\
\text { EPA } 6020 \\
\text { EPA } 6020 \\
\text { EPA } 903.1 \\
\text { EPA } 904.0 \\
\text { HASL } 300 \\
\text { HASL } 300 \\
\text { HASL } 300 \\
\text { EPA } 300.0 \\
\text { EPA } 6020 \\
\text { EPA } 6020 \\
\text { EPA } 903.1 \\
\text { EPA } 904.0 \\
\text { HASL } 300 \\
\text { HASL } 300 \\
\text { HASL } 300 \\
\text { EPA } 300.0 \\
\text { USATHAMA } \\
\text { USATHAMA } \\
\text { USATHAMA } \\
\text { USATHAMA } \\
\text { USATHAMA } \\
\text { USATHAMA } \\
\text { EPA } 903.1 \\
\text { EPA } 904.0 \\
\text { HASL } 300 \\
\text { HASL } 300 \\
\text { HASL } 300 \\
\text { EPA } 6020 \\
\text { EPA } 6020 \\
\text { USATHAMA } \\
\text { USATHAMA } \\
\text { USATHAMA } \\
\text { USATHAMA } \\
\text { USATHAMA } \\
\text { USATHAMA } \\
\text { USATHAMA } \\
\text { USATHAMA } \\
\text { USATHAMA } \\
\text { USATHAMA } \\
\text { USATHAMA } \\
\text { USATHAMA } \\
\text { USATHAMA } \\
\end{array}$ \\
\hline
\end{tabular}




\begin{tabular}{|c|c|c|c|c|c|c|}
\hline USSRAP_ID & DATE_SAM & PARAMETER & CONC & $D L$ & UNITS & METHOD \\
\hline 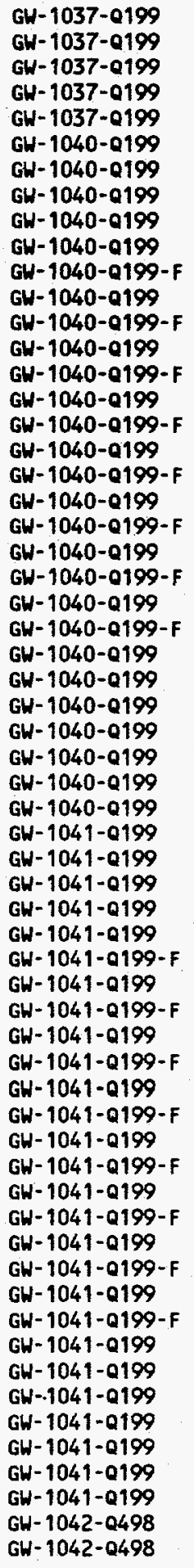 & $\begin{array}{l}01 / 19 / 99 \\
01 / 19 / 99 \\
01 / 19 / 99 \\
01 / 19 / 99 \\
01 / 19 / 99 \\
01 / 15 / 99 \\
01 / 15 / 99 \\
01 / 15 / 99 \\
01 / 15 / 99 \\
01 / 15 / 99 \\
01 / 15 / 99 \\
01 / 15 / 99 \\
01 / 15 / 99 \\
01 / 15 / 99 \\
01 / 15 / 99 \\
01 / 15 / 99 \\
01 / 15 / 99 \\
01 / 15 / 99 \\
01 / 15 / 99 \\
01 / 15 / 99 \\
01 / 15 / 99 \\
01 / 15 / 99 \\
01 / 15 / 99 \\
01 / 15 / 99 \\
01 / 15 / 99 \\
01 / 15 / 99 \\
01 / 15 / 99 \\
01 / 15 / 99 \\
01 / 15 / 99 \\
01 / 15 / 99 \\
01 / 15 / 99 \\
01 / 15 / 99 \\
01 / 15 / 99 \\
01 / 15 / 99 \\
01 / 15 / 99 \\
01 / 15 / 99 \\
01 / 15 / 99 \\
01 / 15 / 99 \\
01 / 15 / 99 \\
01 / 15 / 99 \\
01 / 15 / 99 \\
01 / 15 / 99 \\
01 / 15 / 99 \\
01 / 15 / 99 \\
01 / 15 / 99 \\
01 / 15 / 99 \\
01 / 15 / 99 \\
01 / 15 / 99 \\
01 / 15 / 99 \\
01 / 15 / 99 \\
01 / 15 / 99 \\
01 / 15 / 99 \\
01 / 15 / 99 \\
01 / 15 / 99 \\
01 / 15 / 99 \\
011115 / 99 \\
01 / 15 / 99 \\
01 / 15 / 99 \\
01 / 15 / 99 \\
10 / 20 / 98 \\
10 / 20 / 98\end{array}$ & 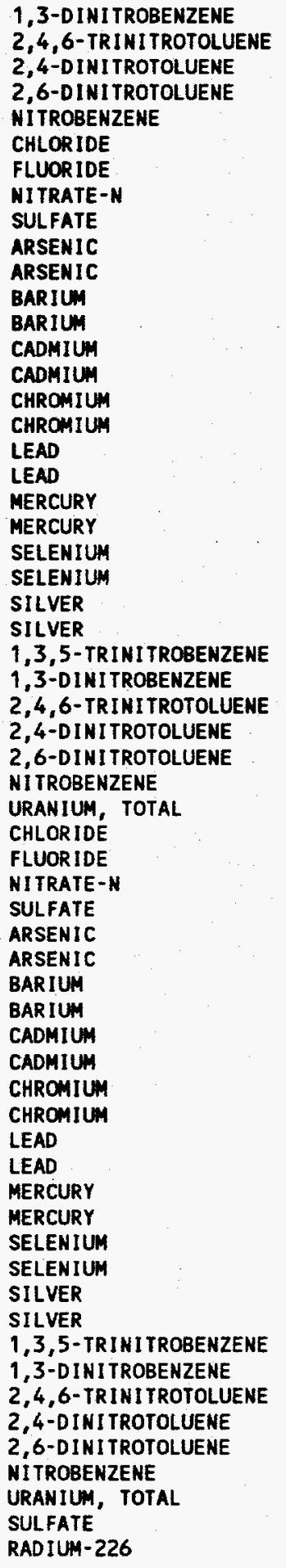 & $\begin{array}{l}\text { ND } \\
\text { ND } \\
\text { ND } \\
\text { ND } \\
\text { ND } \\
24.4 \\
0.111 \\
0.070 \\
162 \\
\text { ND } \\
\text { ND } \\
191 \\
179 \\
\text { ND } \\
\text { ND } \\
1.9 \\
0.82 \\
\text { ND } \\
\text { ND } \\
\text { ND } \\
\text { ND } \\
\text { ND } \\
\text { ND } \\
1.2 \\
\text { ND } \\
\text { ND } \\
\text { ND } \\
\text { ND } \\
\text { ND } \\
\text { ND } \\
\text { ND } \\
9.0 \\
5.11 \\
0.102 \\
0.060 \\
52.2 \\
3.2 \\
2.7 \\
442 \\
370 \\
0.52 \\
\text { ND } \\
8.2 \\
1.9 \\
4.3 \\
\text { ND } \\
\text { ND } \\
\text { ND } \\
\text { ND } \\
\text { ND } \\
\text { ND } \\
\text { ND } \\
\text { ND } \\
\text { ND } \\
\text { ND } \\
\text { ND } \\
\text { ND } \\
\text { ND } \\
6.31 \\
131 \\
0.915\end{array}$ & $\begin{array}{l}0.090 \\
0.030 \\
0.030 \\
0.010 \\
0.030 \\
.0181 \\
.0218 \\
.0086 \\
.152 \\
2.4 \\
2.4 \\
0.1 \\
0.1 \\
0.3 \\
0.3 \\
0.4 \\
0.4 \\
1.4 \\
1.4 \\
0.12 \\
0.12 \\
2.6 \\
2.6 \\
1.1 \\
1.1 \\
0.03 \\
0\end{array}$ & 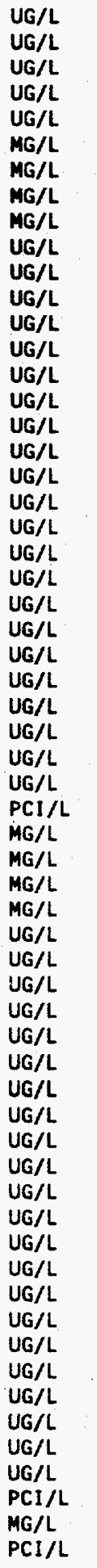 & $\begin{array}{l}\text { USATHAMA } \\
\text { USATHAMA } \\
\text { USATHAMA } \\
\text { USATHAMA } \\
\text { USATHAMA } \\
\text { EPA } 300.0 \\
\text { EPA } 300.0 \\
\text { EPA } 353.1 \\
\text { EPA } 300.0 \\
\text { EPA } 6010 A \\
\text { EPA } 6010 A \\
\text { EPA } 6010 A \\
\text { EPA } 6010 A \\
\text { EPA } 6010 A \\
\text { EPA } 6010 A \\
\text { EPA } 6010 A \\
\text { EPA } 6010 A \\
\text { EPA } 6010 A \\
\text { EPA } 6010 A \\
\text { EPA } 7470 A \\
\text { EPA } 7470 A \\
\text { EPA } 6010 A \\
\text { EPA } 6010 A \\
\text { EPA } 6010 A \\
\text { EPA } 6010 A \\
\text { USATHAMA } \\
\text { USATHAMA } \\
\text { USATHAMA } \\
\text { USATHAMA } \\
\text { USATHAMA } \\
\text { USATHAMA } \\
\text { ASTM } 5174-91 \\
\text { EPA } 300.0 \\
\text { EPA } 300.0 \\
\text { EPA } 353.1 \\
\text { EPA } 300.0 \\
\text { EPA } 6010 A \\
\text { EPA } 6010 A \\
\text { EPA } 6010 A \\
\text { EPA } 6010 A \\
\text { EPA } 6010 A \\
\text { EPA } 6010 A \\
\text { EPA } 6010 A \\
\text { EPA } 6010 A \\
\text { EPA } 6010 A \\
\text { EPA } 6010 A \\
\text { EPA } 7470 A \\
\text { EPA } 7470 A \\
\text { EPA } 6010 A \\
\text { EPA } 6010 A \\
\text { EPA } 6010 A \\
\text { EPA } 6010 A \\
\text { USATHAMA } \\
\text { USATHAMA } \\
\text { USATHAMA } \\
\text { USATHAMA } \\
\text { USATHAMA } \\
\text { USATHAMA } \\
\text { ASTM } 5174-91 \\
\text { EPA } 300.0 \\
\text { EPA } 904.0 \\
\end{array}$ \\
\hline
\end{tabular}




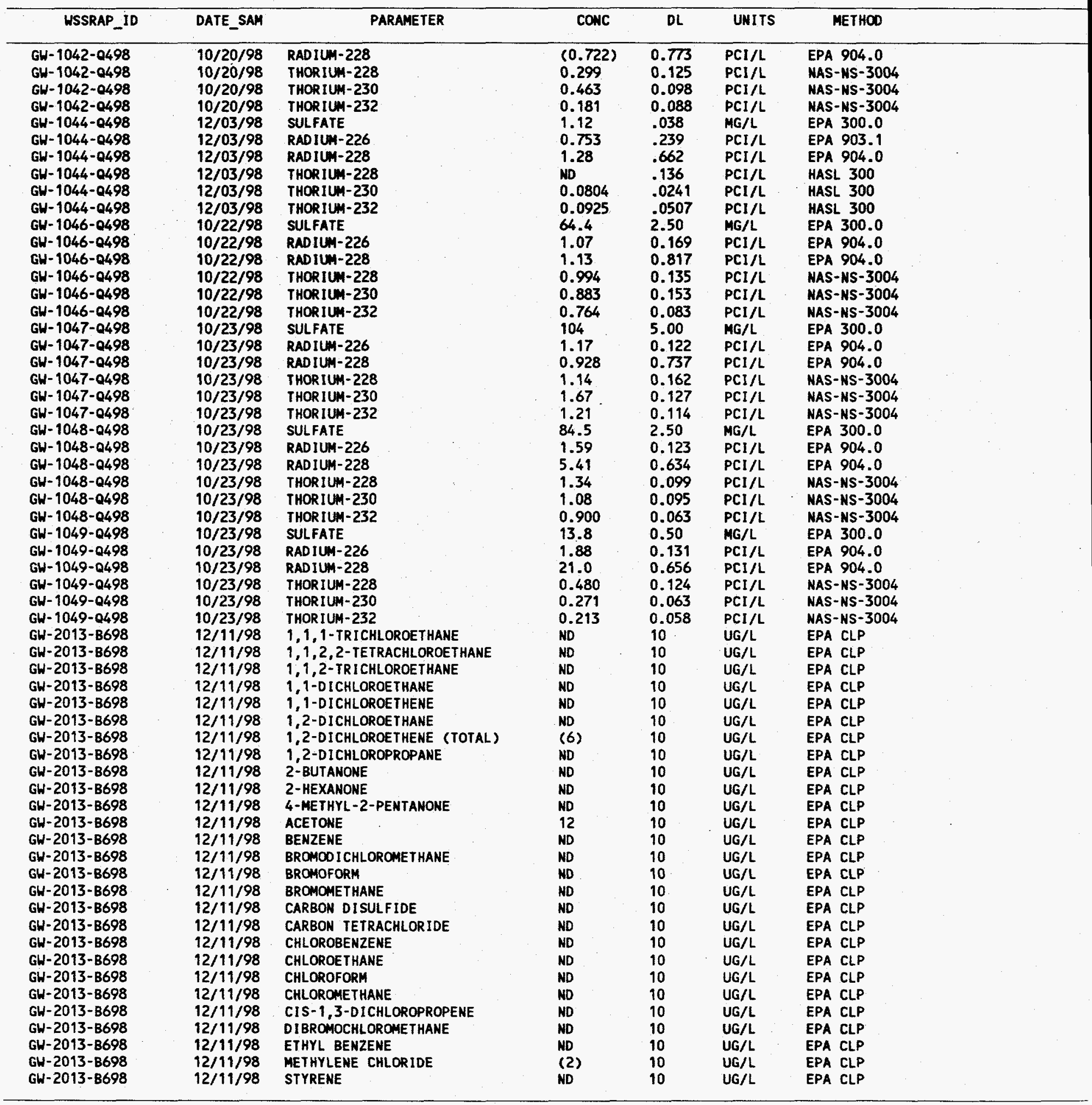




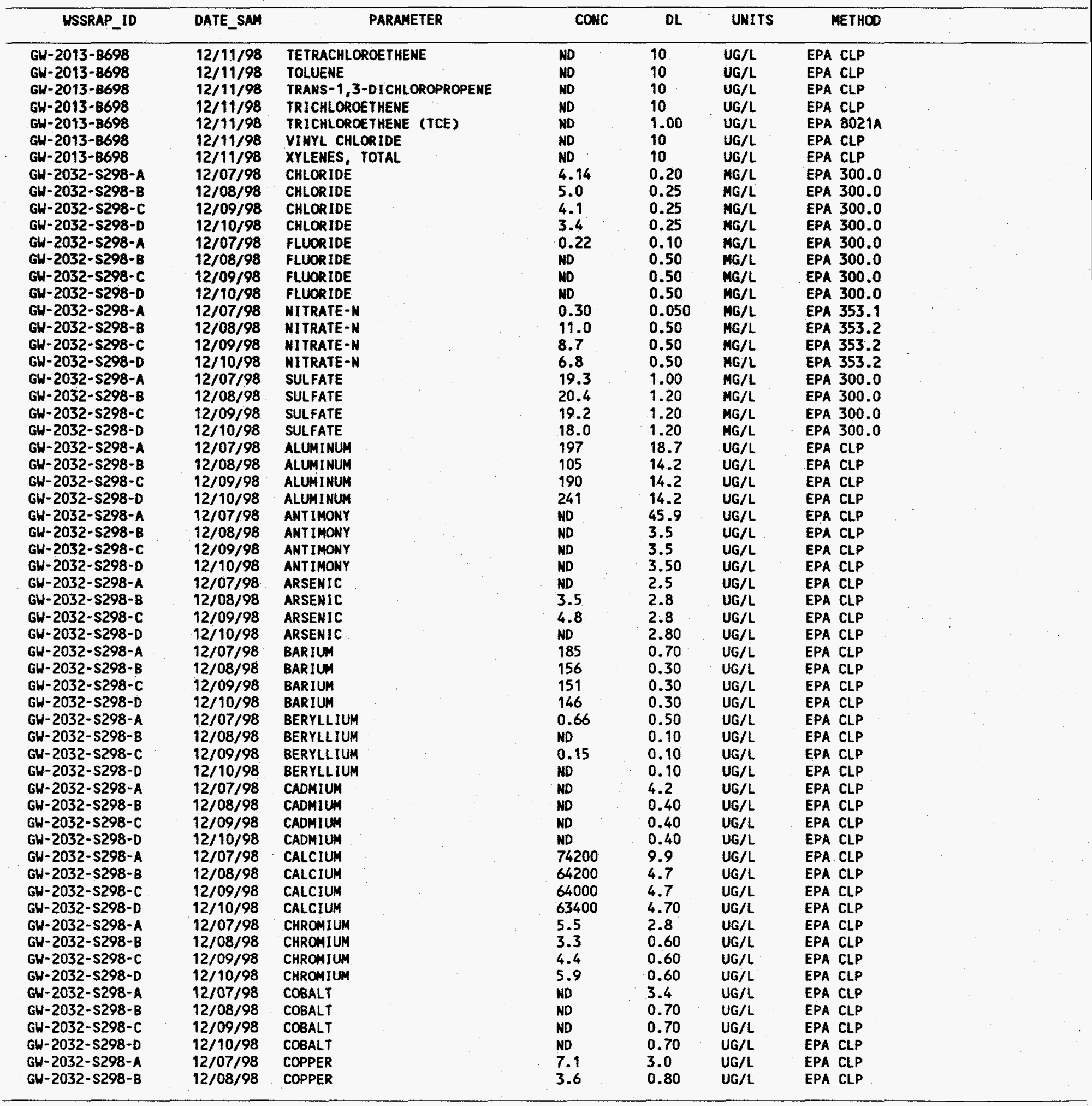




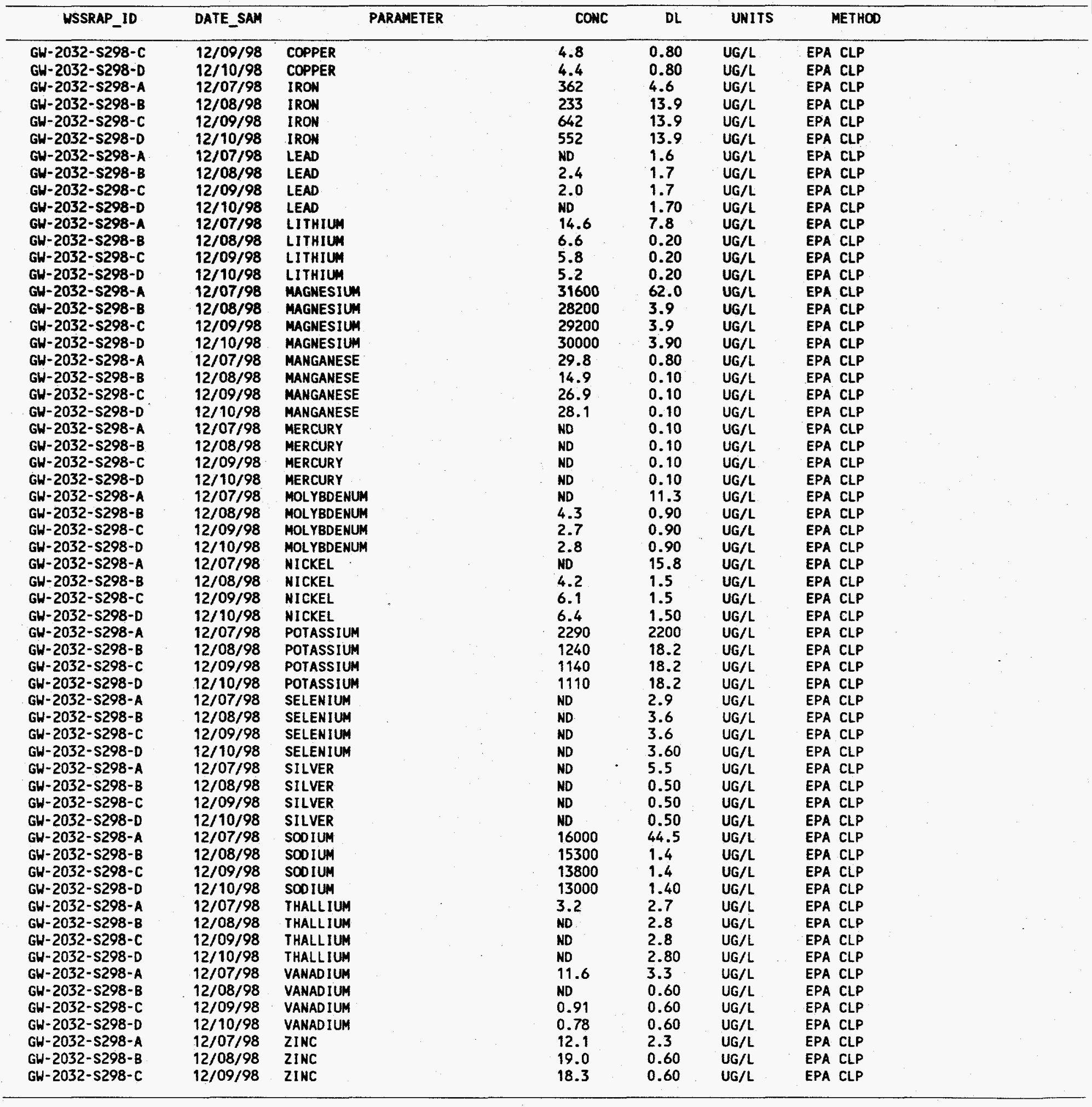




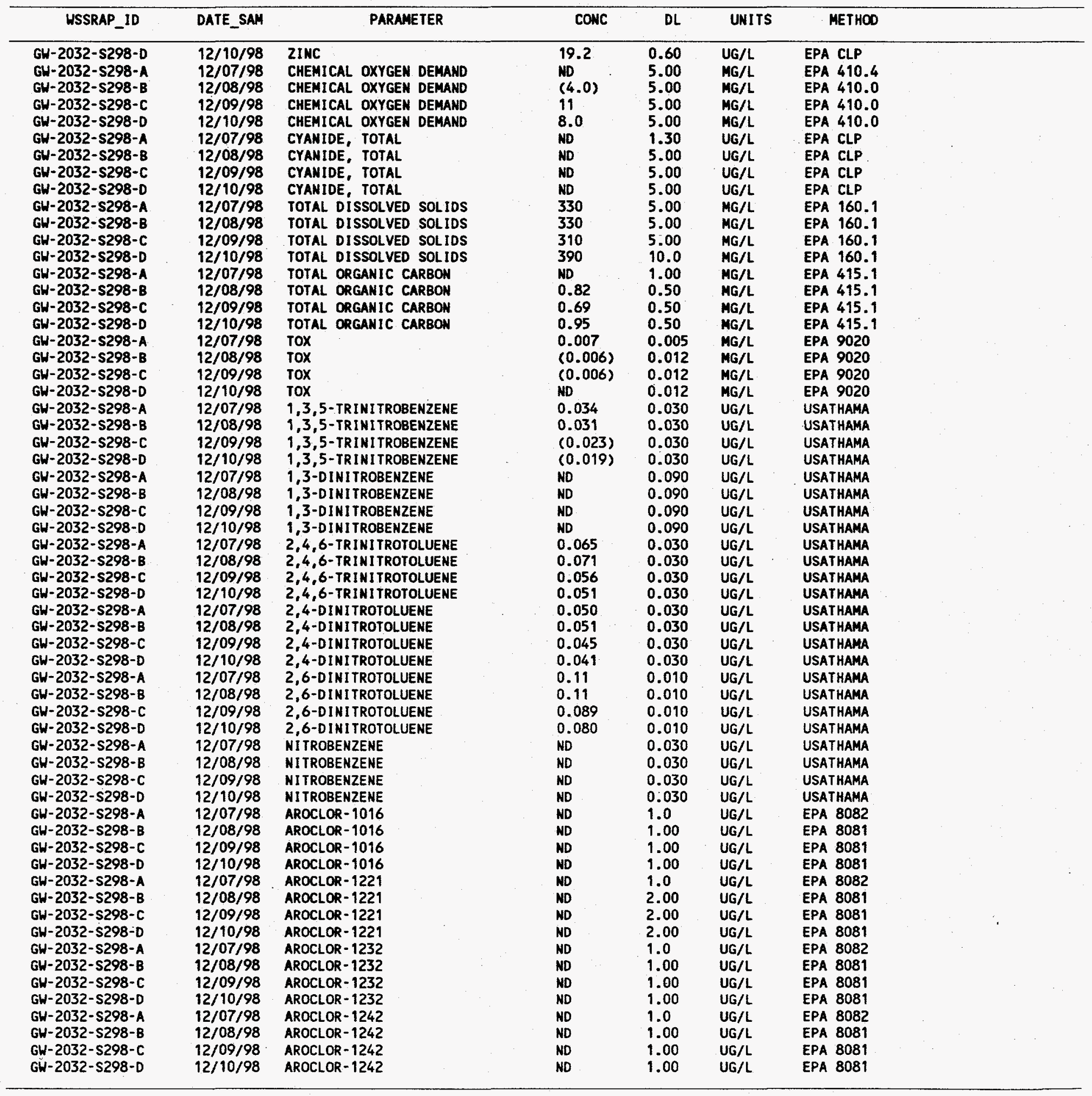




\begin{tabular}{|c|c|c|c|c|c|c|}
\hline HSSRAP_ID & DATE_SAM & PARAMETER & CONC & DL & UNITS & METHOD \\
\hline 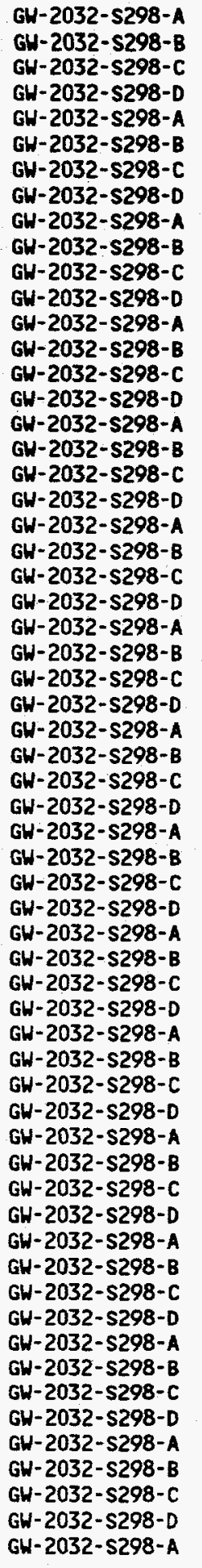 & $\begin{array}{l}12 / 07 / 98 \\
12 / 08 / 98 \\
12 / 09 / 98 \\
12 / 10 / 98 \\
12 / 07 / 98 \\
12 / 08 / 98 \\
12 / 09 / 98 \\
12 / 10 / 98 \\
12 / 07 / 98 \\
12 / 08 / 98 \\
12 / 09 / 98 \\
12 / 10 / 98 \\
12 / 07 / 98 \\
12 / 08 / 98 \\
12 / 09 / 98 \\
12 / 10 / 98 \\
12 / 07 / 98 \\
12 / 08 / 98 \\
12 / 09 / 98 \\
12 / 10 / 98 \\
12 / 07 / 98 \\
12 / 08 / 98 \\
12 / 09 / 98 \\
12 / 10 / 98 \\
12 / 07 / 98 \\
12 / 08 / 98 \\
12 / 09 / 98 \\
12 / 10 / 98 \\
12 / 07 / 98 \\
12 / 08 / 98 \\
12 / 09 / 98 \\
12 / 10 / 98 \\
12 / 07 / 98 \\
12 / 08 / 98 \\
12 / 09 / 98 \\
12 / 10 / 98 \\
12 / 07 / 98 \\
12 / 08 / 98 \\
12 / 09 / 98 \\
12 / 10 / 98 \\
12 / 07 / 98 \\
12 / 08 / 98 \\
12 / 09 / 98 \\
12 / 10 / 98 \\
12 / 07 / 98 \\
12 / 08 / 98 \\
12 / 09 / 98 \\
12 / 10 / 98 \\
12 / 07 / 98 \\
12 / 08 / 98 \\
12 / 09 / 98 \\
12 / 10 / 98 \\
12 / 07 / 98 \\
12 / 08 / 98 \\
12 / 09 / 98 \\
12 / 10 / 98 \\
12 / 07 / 98 \\
12 / 08 / 98 \\
12 / 09 / 98 \\
12 / 10 / 98 \\
12 / 07 / 98\end{array}$ & $\begin{array}{l}\text { AROCLOR-1248 } \\
\text { AROCLOR-1248 } \\
\text { AROCLOR-1248 } \\
\text { AROCLOR-1248 } \\
\text { AROCLOR-1254 } \\
\text { AROCLOR-1254 } \\
\text { AROCLOR-1254 } \\
\text { AROCLOR-1254 } \\
\text { AROCLOR-1260 } \\
\text { AROCLOR-1260 } \\
\text { AROCLOR-1260 } \\
\text { AROCLOR-1260 } \\
\text { RADIUM-226 } \\
\text { RADIUM-226 } \\
\text { RADIUM-226 } \\
\text { RADIUH-226 } \\
\text { RADIUM-228 } \\
\text { RADIUM-228 } \\
\text { RADIUM-228 } \\
\text { RADIUH-228 } \\
\text { THORIUM-228 } \\
\text { THORIUM-228 } \\
\text { THORIUM-228 } \\
\text { THORIUM-228 } \\
\text { THORIUM-230 } \\
\text { THORIUM-230 } \\
\text { THORIUM-230 } \\
\text { THORIUM-230 } \\
\text { THORIUM-232 } \\
\text { THORIUM-232 } \\
\text { THORIUM-232 } \\
\text { THORIUM-232 } \\
\text { URANIUM, TOTAL } \\
\text { URANIUM, TOTAL } \\
\text { URANIUM, TOTAL } \\
\text { URANIUM, TOTAL } \\
\text { ACENAPHTHENE } \\
\text { ACENAPHTHENE } \\
\text { ACENAPHTHENE } \\
\text { ACENAPHTHENE } \\
\text { ACENAPHTHYLENE } \\
\text { ACENAPHTHYLENE } \\
\text { ACENAPHTHYLENE } \\
\text { ACENAPHTHYLENE } \\
\text { ANTHRACENE } \\
\text { ANTHRACENE } \\
\text { ANTHRACENE } \\
\text { ANTHRACENE } \\
\text { BENZO(A)ANTHRACENE } \\
\text { BENZO(A)ANTHRACENE } \\
\text { BENZO(A)ANTHRACENE } \\
\text { BENZO(A)ANTHRACENE } \\
\text { BENZO(A)PYRENE } \\
\text { BENZO(A)PYRENE } \\
\text { BENZO(A)PYRENE } \\
\text { BENZO(A)PYRENE } \\
\text { BENZO(B) FLUORANTHENE } \\
\text { BENZO(B) FLUORANTHENE } \\
\text { BENZO(B) FLUORANTHENE } \\
\text { BENZO(B)FLUORANTHENE } \\
\text { BENZO(G, H, I)PERYLENE }\end{array}$ & 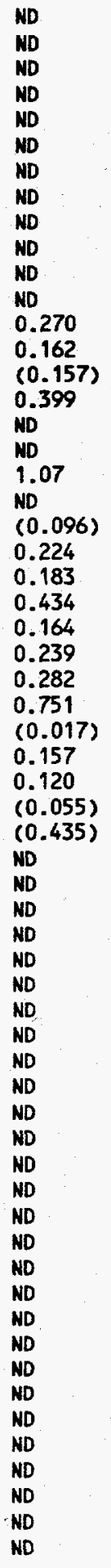 & $\begin{array}{l}1.0 \\
1.00 \\
1.00 \\
1.00 \\
1.0 \\
1.00 \\
1.00 \\
1.00 \\
1.0 \\
1.00 \\
1.00 \\
1.00 \\
0.111 \\
0.109 \\
0.158 \\
0.156 \\
0.469 \\
0.469 \\
0.469 \\
0.469 \\
0.104 \\
0.103 \\
0.112 \\
0.168 \\
0.080 \\
0.048 \\
0.082 \\
0.147 \\
0.087 \\
0.095 \\
0.090 \\
0.147 \\
0.677 \\
0.677 \\
0.677 \\
0.677 \\
5.0 \\
18 \\
18 \\
18.0 \\
5.0 \\
23 \\
23 \\
23.0 \\
5.0 \\
6.6 \\
6.6 \\
6.60 \\
5.0 \\
0.13 \\
0.13 \\
0.13 \\
5.0 \\
0.23 \\
0.23 \\
0.23 \\
5.0 \\
0.18 \\
0.18 \\
0.18 \\
5.0 \\
\end{array}$ & $\begin{array}{l}U G / L \\
U G / L \\
U G / L \\
U G / L \\
U G / L \\
U G / L \\
U G / L \\
U G / L \\
U G / L \\
U G / L \\
U G / L \\
U G / L \\
P C I / L \\
P C I / L \\
P C I / L \\
P C I / G \\
P C I / L \\
P C I / L \\
P C I / L \\
P C I / G \\
P C I / L \\
P C I / L \\
P C I / L \\
P C I / G \\
P C I / L \\
P C I / L \\
P C I / L \\
P C I / G \\
P C I / L \\
P C I / L \\
P C I / L \\
P C I / G \\
P C I / L \\
P C I / L \\
P C I / L \\
P C I / G \\
U G / L \\
U G / L \\
U G / L \\
U G / L \\
U G / L \\
U G / L \\
U G / L \\
U G / L \\
U G / L \\
U G / L \\
U G / L \\
U G / L \\
U G / L \\
U G / L \\
U G / L \\
U G / L \\
U G / L \\
U G / L \\
U G / L \\
U G / L \\
U G / L \\
U G / L \\
U G / L \\
U G / L \\
U G / L \\
\end{array}$ & $\begin{array}{l}\text { EPA } 8082 \\
\text { EPA } 8081 \\
\text { EPA } 8081 \\
\text { EPA } 8081 \\
\text { EPA } 8082 \\
\text { EPA } 8081 \\
\text { EPA } 8081 \\
\text { EPA } 8081 \\
\text { EPA } 8082 \\
\text { EPA } 8081 \\
\text { EPA } 8081 \\
\text { EPA } 8081 \\
\text { SM-705M } \\
\text { SM-705M } \\
\text { SM-705M } \\
\text { SH-705M } \\
\text { EPA } 9320 \\
\text { EPA } 9320 \\
\text { EPA } 9320 \\
\text { EPA } 9320 \\
\text { LANL ER200 } \\
\text { LANL ER200 } \\
\text { LANL ER200 } \\
\text { LANL ER200 } \\
\text { LANL ER200 } \\
\text { LANL ER200 } \\
\text { LANL ER200 } \\
\text { LANL ER200 } \\
\text { LANL ER200 } \\
\text { LANL ER200 } \\
\text { LANL ER200 } \\
\text { LANL ER200 } \\
\text { ASTM } 5174-91 \\
\text { ASTM } 5174-91 \\
\text { ASTM } 5174-91 \\
\text { ASTM } 5174-91 \\
\text { EPA } 8310 \\
\text { EPA } 8310 \\
\text { EPA } 8310 \\
\text { EPA } 8310 \\
\text { EPA } 8310 \\
\text { EPA } 8310 \\
\text { EPA } 8310 \\
\text { EPA } 8310 \\
\text { EPA } 8310 \\
\text { EPA } 8310 \\
\text { EPA } 8310 \\
\text { EPA } 8310 \\
\text { EPA } 8310 \\
\text { EPA } 8310 \\
\text { EPA } 8310 \\
\text { EPA } 8310 \\
\text { EPA } 8310 \\
\text { EPA } 8310 \\
\text { EPA } 8310 \\
\text { EPA } 8310 \\
\text { EPA } 8310 \\
\text { EPA } 8310 \\
\text { EPA } 8310 \\
\text { EPA } 8310 \\
\text { EPA } 8310 \\
\end{array}$ \\
\hline
\end{tabular}




\begin{tabular}{|c|c|c|c|c|c|c|}
\hline USSRAP_ID & DATE_SAM & PARAMETER & CONC & $D L$ & UNITS & METHOD \\
\hline 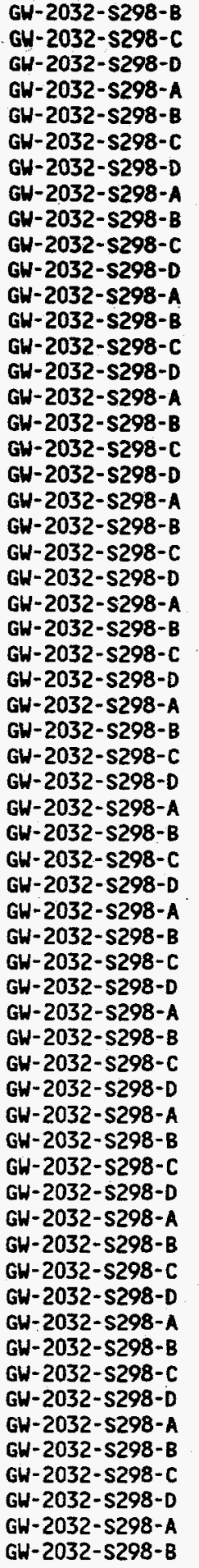 & $\begin{array}{l}12 / 08 / 98 \\
12 / 09 / 98 \\
12 / 10 / 98 \\
12 / 07 / 98 \\
12 / 08 / 98 \\
12 / 09 / 98 \\
12 / 10 / 98 \\
12 / 07 / 98 \\
12 / 08 / 98 \\
12 / 09 / 98 \\
12 / 10 / 98 \\
12 / 07 / 98 \\
12 / 08 / 98 \\
12 / 09 / 98 \\
12 / 10 / 98 \\
12 / 07 / 98 \\
12 / 08 / 98 \\
12 / 09 / 98 \\
12 / 10 / 98 \\
12 / 07 / 98 \\
12 / 08 / 98 \\
12 / 09 / 98 \\
12 / 10 / 98 \\
12 / 07 / 98 \\
12 / 08 / 98 \\
12 / 09 / 98 \\
12 / 10 / 98 \\
12 / 07 / 98 \\
12 / 08 / 98 \\
12 / 09 / 98 \\
12 / 10 / 98 \\
12 / 07 / 98 \\
12 / 08 / 98 \\
12 / 09 / 98 \\
12 / 10 / 98 \\
12 / 07 / 98 \\
12 / 08 / 98 \\
12 / 09 / 98 \\
12 / 10 / 98 \\
12 / 07 / 98 \\
12 / 08 / 98 \\
12 / 09 / 98 \\
12 / 10 / 98 \\
12 / 07 / 98 \\
12 / 08 / 98 \\
12 / 09 / 98 \\
12 / 10 / 98 \\
12 / 07 / 98 \\
12 / 08 / 98 \\
12 / 09 / 98 \\
12 / 10 / 98 \\
12 / 07 / 98 \\
12 / 08 / 98 \\
12 / 09 / 98 \\
12 / 10 / 98 \\
12 / 07 / 98 \\
12 / 08 / 98 \\
12 / 09 / 98 \\
12 / 10 / 98 \\
12 / 07 / 98 \\
12 / 08 / 98\end{array}$ & 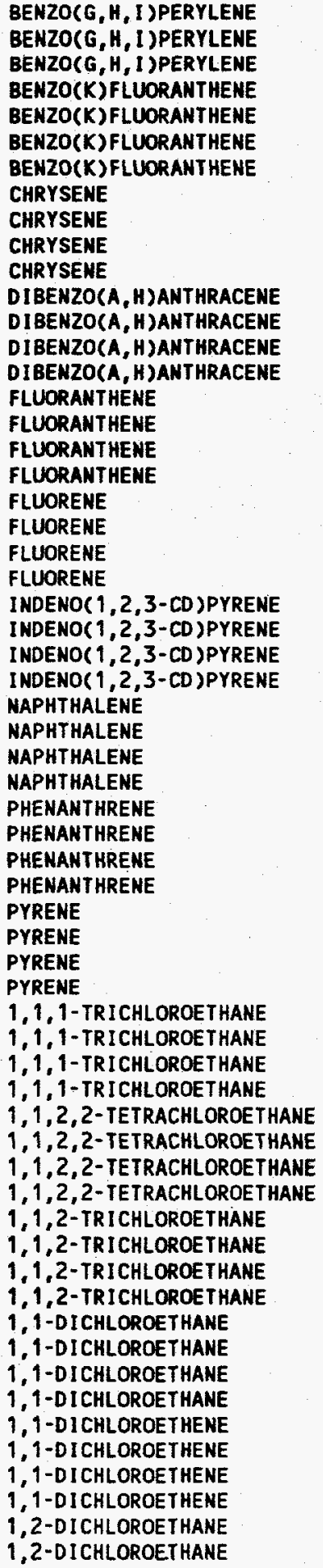 & $\begin{array}{l}\text { ND } \\
\text { ND } \\
\text { ND } \\
\text { ND } \\
\text { ND } \\
\text { ND } \\
\text { ND } \\
\text { ND } \\
\text { ND } \\
\text { ND } \\
\text { ND } \\
\text { ND } \\
\text { ND } \\
\text { ND } \\
\text { ND } \\
\text { ND } \\
\text { ND } \\
\text { ND } \\
\text { ND } \\
\text { ND } \\
\text { ND } \\
\text { ND } \\
\text { ND } \\
\text { ND } \\
\text { ND } \\
\text { ND } \\
\text { ND } \\
\text { ND } \\
\text { ND } \\
\text { ND } \\
\text { ND } \\
\text { ND } \\
\text { ND } \\
\text { ND } \\
\text { ND } \\
\text { ND } \\
\text { ND } \\
\text { ND } \\
\text { ND } \\
\text { ND } \\
\text { ND } \\
\text { ND } \\
\text { ND } \\
\text { ND } \\
\text { ND } \\
\text { ND } \\
\text { ND } \\
\text { ND } \\
\text { ND } \\
\text { ND } \\
\text { ND } \\
\text { ND } \\
\text { ND } \\
\text { ND } \\
\text { ND } \\
\text { ND } \\
\text { ND } \\
\text { ND } \\
\text { ND } \\
\text { ND } \\
\text { ND }\end{array}$ & $\begin{array}{l}0.76 \\
0.76 \\
0.76 \\
5.0 \\
0.17 \\
0.17 \\
0.17 \\
5.0 \\
1.5 \\
1.5 \\
1.50 \\
5.0 \\
0.30 \\
0.30 \\
0.30 \\
5.0 \\
2.1 \\
2.1 \\
2.10 \\
5.0 \\
2.1 \\
2.1 \\
2.10 \\
5.0 \\
0.43 \\
0.43 \\
0.43 \\
5.0 \\
18 \\
18 \\
18.0 \\
5.0 \\
6.4 \\
6.4 \\
6.40 \\
5.0 \\
2.7 \\
2.7 \\
2.70 \\
10 \\
10 \\
10 \\
10 \\
10 \\
10 \\
10 \\
10 \\
10 \\
10 \\
10 \\
10 \\
10 \\
10 \\
10 \\
10 \\
10 \\
10 \\
10 \\
10 \\
10 \\
10 \\
\end{array}$ & $\begin{array}{l}U G / L \\
U G / L \\
U G / L \\
U G / L \\
U G / L \\
U G / L \\
U G / L \\
U G / L \\
U G / L \\
U G / L \\
U G / L \\
U G / L \\
U G / L \\
U G / L \\
U G / L \\
U G / L \\
U G / L \\
U G / L \\
U G / L \\
U G / L \\
U G / L \\
U G / L \\
U G / L \\
U G / L \\
U G / L \\
U G / L \\
U G / L \\
U G / L \\
U G / L \\
U G / L \\
U G / L \\
U G / L \\
U G / L \\
U G / L \\
U G / L \\
U G / L \\
U G / L \\
U G / L \\
U G / L \\
U G / L \\
U G / L \\
U G / L \\
U G / L \\
U G / L \\
U G / L \\
U G / L \\
U G / L \\
U G / L \\
U G / L \\
U G / L \\
U G / L \\
U G / L \\
U G / L \\
U G / L \\
U G / L \\
U G / L \\
U G / L \\
U G / L \\
U G / L \\
U G \\
U\end{array}$ & 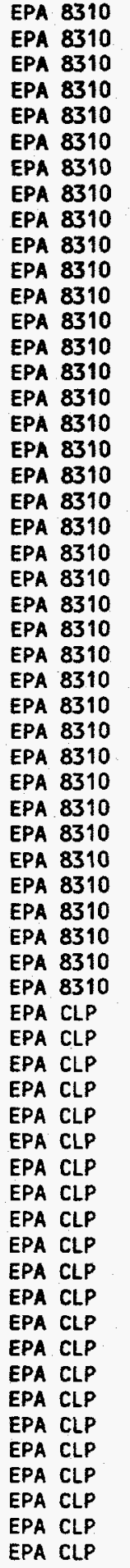 \\
\hline
\end{tabular}




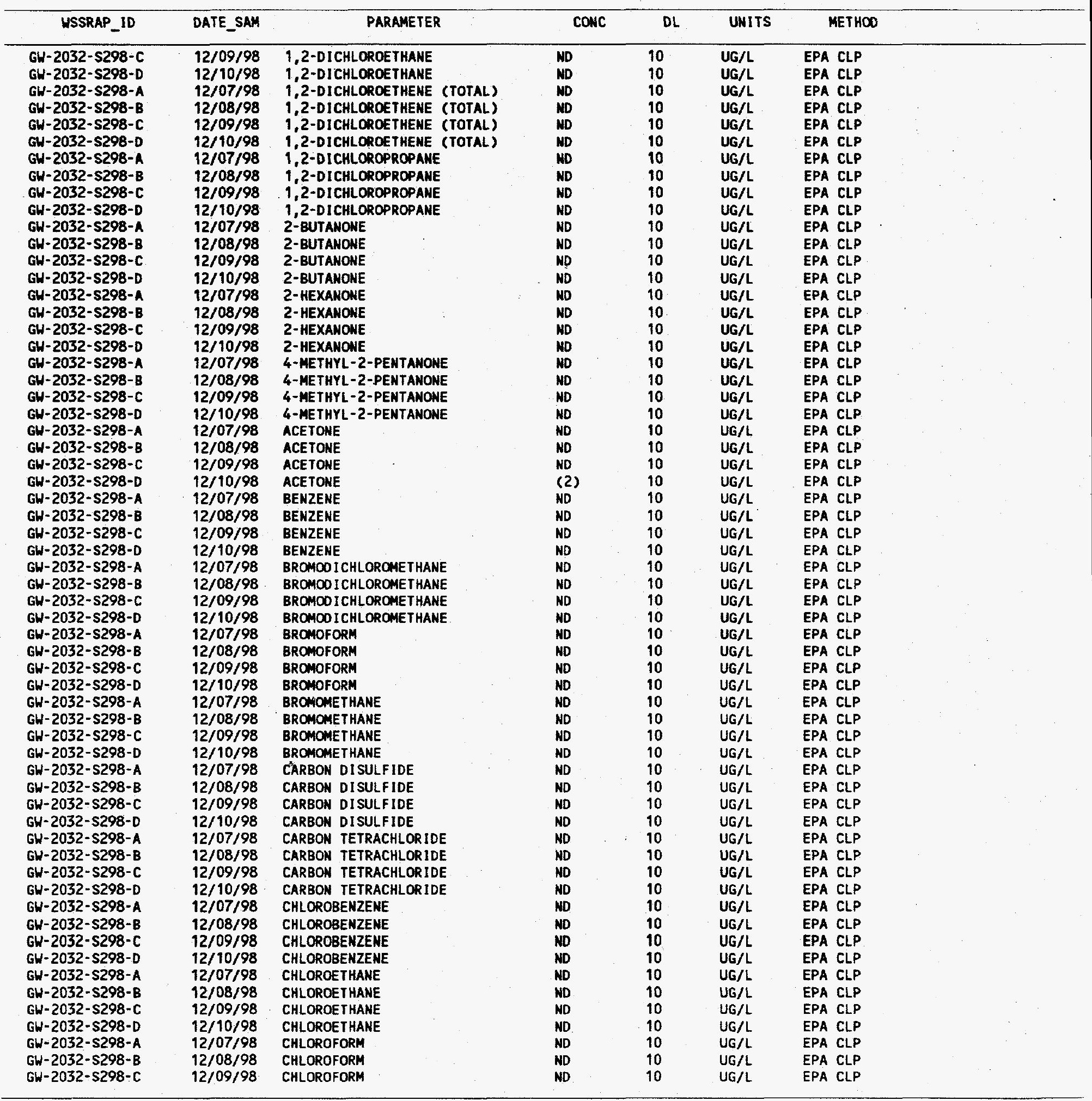




\begin{tabular}{|c|c|c|c|c|c|c|c|}
\hline WSSRAP_ID & DATE_SAM & PARAMETER & CONC & DL & UNITS & METHOD & \\
\hline $\begin{array}{l}G W-2032-S 298-D \\
G H-2032-S 298-A \\
G W-2032-S 298-B \\
G W-2032-S 298-C \\
G W-2032-S 298-D \\
G W-2032-S 298-A \\
G W-2032-S 298-B \\
G W-2032-S 298-C \\
G W-2032-S 298-D \\
G W-2032-S 298-A \\
G W-2032-S 298-B \\
G W-2032-S 298-C \\
G W-2032-S 298-D \\
G W-2032-S 298-A \\
G W-2032-S 298-B \\
G W-2032-S 298-C \\
G W-2032-S 298-D \\
G W-2032-S 298-A \\
G W-2032-S 298-B \\
G W-2032-S 298-C \\
G W-2032-S 298-D \\
G W-2032-S 298-C \\
G W-2032-S 298-A \\
G W-2032-S 298-B \\
G W-2032-S 298-C \\
G W-2032-S 298-D \\
G W-2032-S 298-A \\
G W-2032-S 298-B \\
G W-2032-S 298-C \\
G W-2032-S 298-D \\
G W-2032-S 298-A \\
G W-2032-S 298-B \\
G W-2032-S 298-C \\
G W-2032-S 298-D \\
G W-2032-S 298-A \\
G W-2032-S 298-B \\
G W-2032-S 298-C \\
G W-2032-S 298-D \\
G W-2032-S 298-A \\
G W-2032-S 298-B \\
G W-2032-S 298-C \\
G W-2032-S 298-D \\
G W-2032-S 298-A \\
G W-2032-S 298-A \\
G W-2032-S 298-B \\
G W-2032-S 298-C \\
G W-2032-S 298-D \\
G W-2032-S 298-A \\
G W-2032-S 298-B \\
G W-2032-S 298-C \\
G W-2032-S 298-D \\
G W-2037-B 698 \\
G W-2037-B 698 \\
G W-2037-B 698 \\
G W-2037-B 698 \\
G W-2037-B 698 \\
G W-2037-B 698-F \\
G W-2037-B 698 \\
G W-2037-B 698-F \\
G W-2037-B 698 \\
G W-2037-B 698-F\end{array}$ & $\begin{array}{l}12 / 10 / 98 \\
12 / 07 / 98 \\
12 / 08 / 98 \\
12 / 09 / 98 \\
12 / 10 / 98 \\
12 / 07 / 98 \\
12 / 08 / 98 \\
12 / 09 / 98 \\
12 / 10 / 98 \\
12 / 07 / 98 \\
12 / 08 / 98 \\
12 / 09 / 98 \\
12 / 10 / 98 \\
12 / 07 / 98 \\
12 / 08 / 98 \\
12 / 09 / 98 \\
12 / 10 / 98 \\
12 / 07 / 98 \\
12 / 08 / 98 \\
12 / 09 / 98 \\
12 / 10 / 98 \\
12 / 09 / 98 \\
12 / 07 / 98 \\
12 / 08 / 98 \\
12 / 09 / 98 \\
12 / 10 / 98 \\
12 / 07 / 98 \\
12 / 08 / 98 \\
12 / 09 / 98 \\
12 / 10 / 98 \\
12 / 07 / 98 \\
12 / 08 / 98 \\
12 / 09 / 98 \\
12 / 10 / 98 \\
12 / 07 / 98 \\
12 / 08 / 98 \\
12 / 09 / 98 \\
12 / 10 / 98 \\
12 / 07 / 98 \\
12 / 08 / 98 \\
12 / 09 / 98 \\
12 / 10 / 98 \\
12 / 07 / 98 \\
12 / 07 / 98 \\
12 / 08 / 98 \\
12 / 09 / 98 \\
12 / 10 / 98 \\
12 / 07 / 98 \\
12 / 08 / 98 \\
12 / 09 / 98 \\
12 / 10 / 98 \\
12 / 15 / 98 \\
12 / 15 / 98 \\
12 / 15 / 98 \\
12 / 15 / 98 \\
12 / 15 / 98 \\
12 / 15 / 98 \\
12 / 15 / 98 \\
12 / 15 / 98 \\
12 / 15 / 98 \\
12 / 15 / 98\end{array}$ & 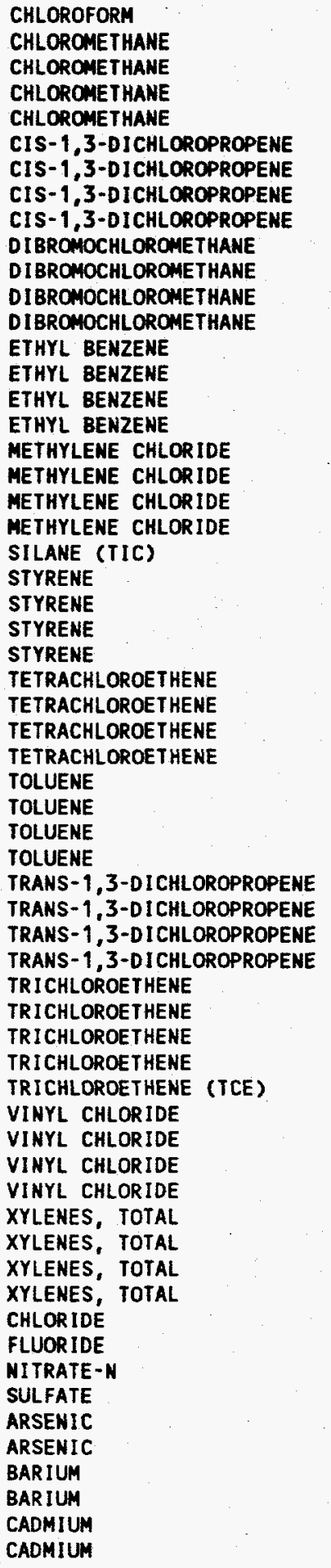 & 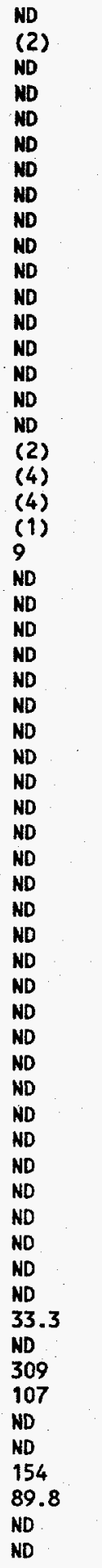 & 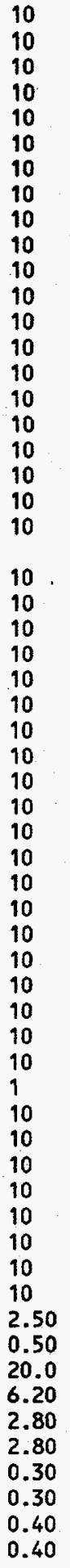 & 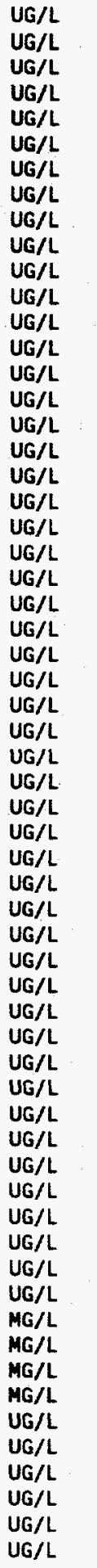 & 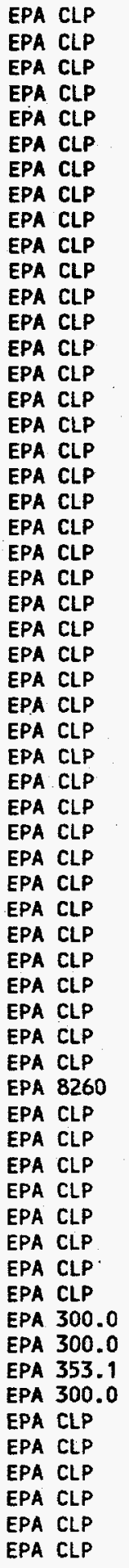 & ' \\
\hline
\end{tabular}




\begin{tabular}{|c|c|c|c|c|c|c|}
\hline WSSRAP_ID & DAIE_SAM & PARAMETER & CONC & DL & UNITS & METHOD \\
\hline 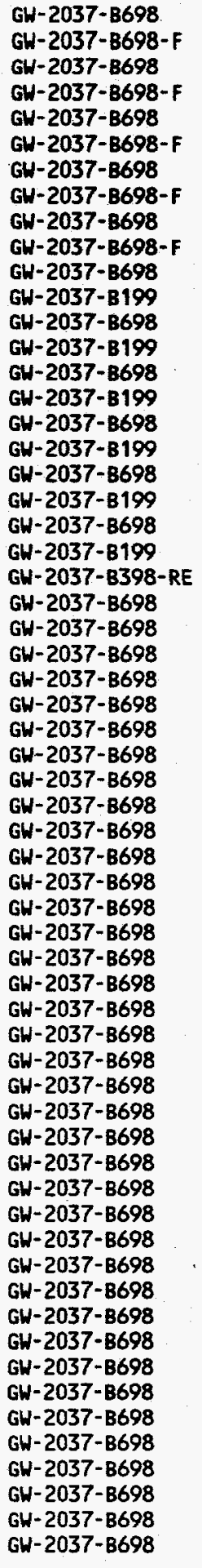 & $\begin{array}{l}12 / 15 / 98 \\
12 / 15 / 98 \\
12 / 15 / 98 \\
12 / 15 / 98 \\
12 / 15 / 98 \\
12 / 15 / 98 \\
12 / 15 / 98 \\
12 / 15 / 98 \\
12 / 15 / 98 \\
12 / 15 / 98 \\
12 / 15 / 98 \\
01 / 20 / 99 \\
12 / 15 / 98 \\
01 / 20 / 99 \\
12 / 15 / 98 \\
01 / 20 / 99 \\
12 / 15 / 98 \\
01 / 20 / 99 \\
12 / 15 / 98 \\
01 / 20 / 99 \\
12 / 15 / 98 \\
01 / 20 / 99 \\
06 / 02 / 98 \\
12 / 15 / 98 \\
12 / 15 / 98 \\
12 / 15 / 98 \\
12 / 15 / 98 \\
12 / 15 / 98 \\
12 / 15 / 98 \\
12 / 15 / 98 \\
12 / 15 / 98 \\
12 / 15 / 98 \\
12 / 15 / 98 \\
12 / 15 / 98 \\
12 / 15 / 98 \\
12 / 15 / 98 \\
12 / 15 / 98 \\
12 / 15 / 98 \\
12 / 15 / 98 \\
12 / 15 / 98 \\
12 / 15 / 98 \\
12 / 15 / 98 \\
12 / 15 / 98 \\
12 / 15 / 98 \\
12 / 15 / 98 \\
12 / 15 / 98 \\
12 / 15 / 98 \\
12 / 15 / 98 \\
12 / 15 / 98 \\
12 / 15 / 98 \\
12 / 15 / 98 \\
12 / 15 / 98 \\
12 / 15 / 98 \\
12 / 15 / 98 \\
12 / 15 / 98 \\
12 / 15 / 98 \\
12 / 15 / 98 \\
12 / 15 / 98 \\
12 / 15 / 98 \\
12 / 15 / 98 \\
12 / 15 / 98\end{array}$ & 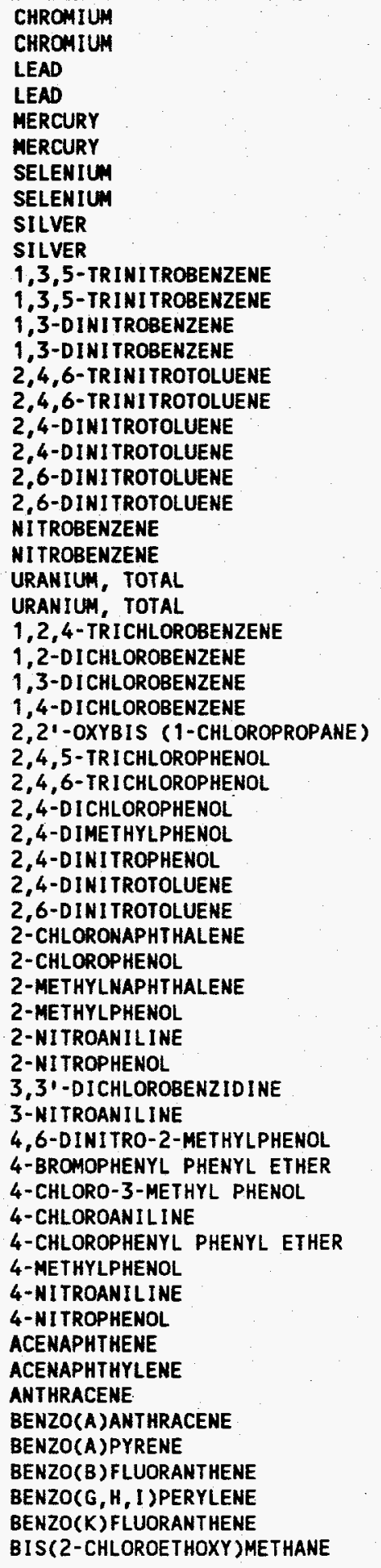 & $\begin{array}{l}7.0 \\
\text { ND } \\
\text { ND } \\
\text { ND } \\
\text { ND } \\
\text { ND } \\
6.8 \\
5.2 \\
1.5 \\
\text { ND } \\
\text { ND } \\
\text { ND } \\
\text { ND } \\
\text { ND } \\
\text { ND } \\
\text { ND } \\
\text { O.17 } \\
\text { O.16 } \\
\text { O.O29 } \\
\text { O.025 } \\
\text { ND } \\
\text { ND } \\
4.22 \\
1.14 \\
\text { ND } \\
\text { ND } \\
\text { ND } \\
\text { ND } \\
\text { ND } \\
\text { ND } \\
\text { ND } \\
\text { ND } \\
\text { ND } \\
\text { ND } \\
\text { ND } \\
\text { ND } \\
\text { ND } \\
\text { ND } \\
\text { ND } \\
\text { ND } \\
\text { ND } \\
\text { ND } \\
\text { ND } \\
\text { ND } \\
\text { ND } \\
\text { ND } \\
\text { ND } \\
\text { ND } \\
\text { ND } \\
\text { ND } \\
\text { ND } \\
\text { ND } \\
\text { ND } \\
\text { ND } \\
\text { ND } \\
\text { ND } \\
\text { ND } \\
\text { ND } \\
\text { ND } \\
\text { ND } \\
\text { ND } \\
\end{array}$ & $\begin{array}{l}0.60 \\
0.60 \\
1.70 \\
1.70 \\
0.10 \\
0.10 \\
3.60 \\
3.60 \\
0.50 \\
0.50 \\
0.030 \\
0.030 \\
0.090 \\
0.090 \\
0.030 \\
0.030 \\
0.030 \\
0.030 \\
0.010 \\
0.010 \\
0.030 \\
0.030 \\
0.6 \\
0.064 \\
10 \\
10 \\
10 \\
10 \\
10 \\
25 \\
10 \\
10 \\
10 \\
25 \\
10 \\
10 \\
10 \\
10 \\
10 \\
10 \\
25 \\
10 \\
10 \\
25 \\
25 \\
10 \\
10 \\
10 \\
10 \\
10 \\
25 \\
25 \\
10 \\
10 \\
10 \\
10 \\
10 \\
10 \\
10 \\
10 \\
10 \\
0\end{array}$ & 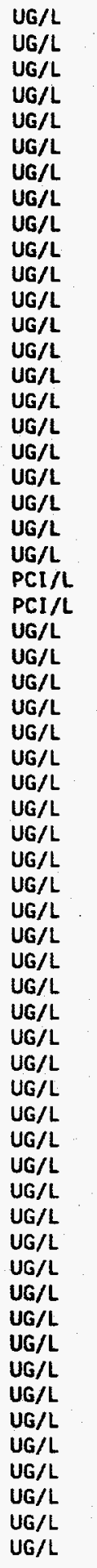 & $\begin{array}{l}\text { EPA CLP } \\
\text { EPA CLP } \\
\text { EPA CLP } \\
\text { EPA CLP } \\
\text { EPA CLP } \\
\text { EPA CLP } \\
\text { EPA CLP } \\
\text { EPA CLP } \\
\text { EPA CLP } \\
\text { EPA CLP } \\
\text { USATHAMA } \\
\text { USATHAMA } \\
\text { USATHAMA } \\
\text { USATHAMA } \\
\text { USATHAMA } \\
\text { USATHAMA } \\
\text { USATHAMA } \\
\text { USATHAMA } \\
\text { USATHAMA } \\
\text { USATHAMA } \\
\text { USATHAMA } \\
\text { USATHAMA } \\
\text { ASTM D2907 } \\
\text { ASTM } 5174-91 \\
\text { EPA CLP } \\
\text { EPA CLP } \\
\text { EPA CLP } \\
\text { EPA CLP } \\
\text { EPA CLP } \\
\text { EPA CLP } \\
\text { EPA CLP } \\
\text { EPA CLP } \\
\text { EPA CLP } \\
\text { EPA CLP } \\
\text { EPA CLP } \\
\text { EPA CLP } \\
\text { EPA CLP } \\
\text { EPA CLP } \\
\text { EPA CLP } \\
\text { EPA CLP } \\
\text { EPA CLP } \\
\text { EPA CLP } \\
\text { EPA CLP } \\
\text { EPA CLP } \\
\text { EPA CLP } \\
\text { EPA CLP } \\
\text { EPA CLP } \\
\text { EPA CLP } \\
\text { EPA CLP } \\
\text { EPA CLP } \\
\text { EPA CLP } \\
\text { EPA CLP } \\
\text { EPA CLP } \\
\text { EPA CLP } \\
\text { EPA CLP } \\
\text { EPA CLP } \\
\text { EPA CLP } \\
\text { EPA CLP } \\
\text { EPA CLP } \\
\text { EPA CLP } \\
\text { EPA CLP } \\
\text { EPA }\end{array}$ \\
\hline
\end{tabular}




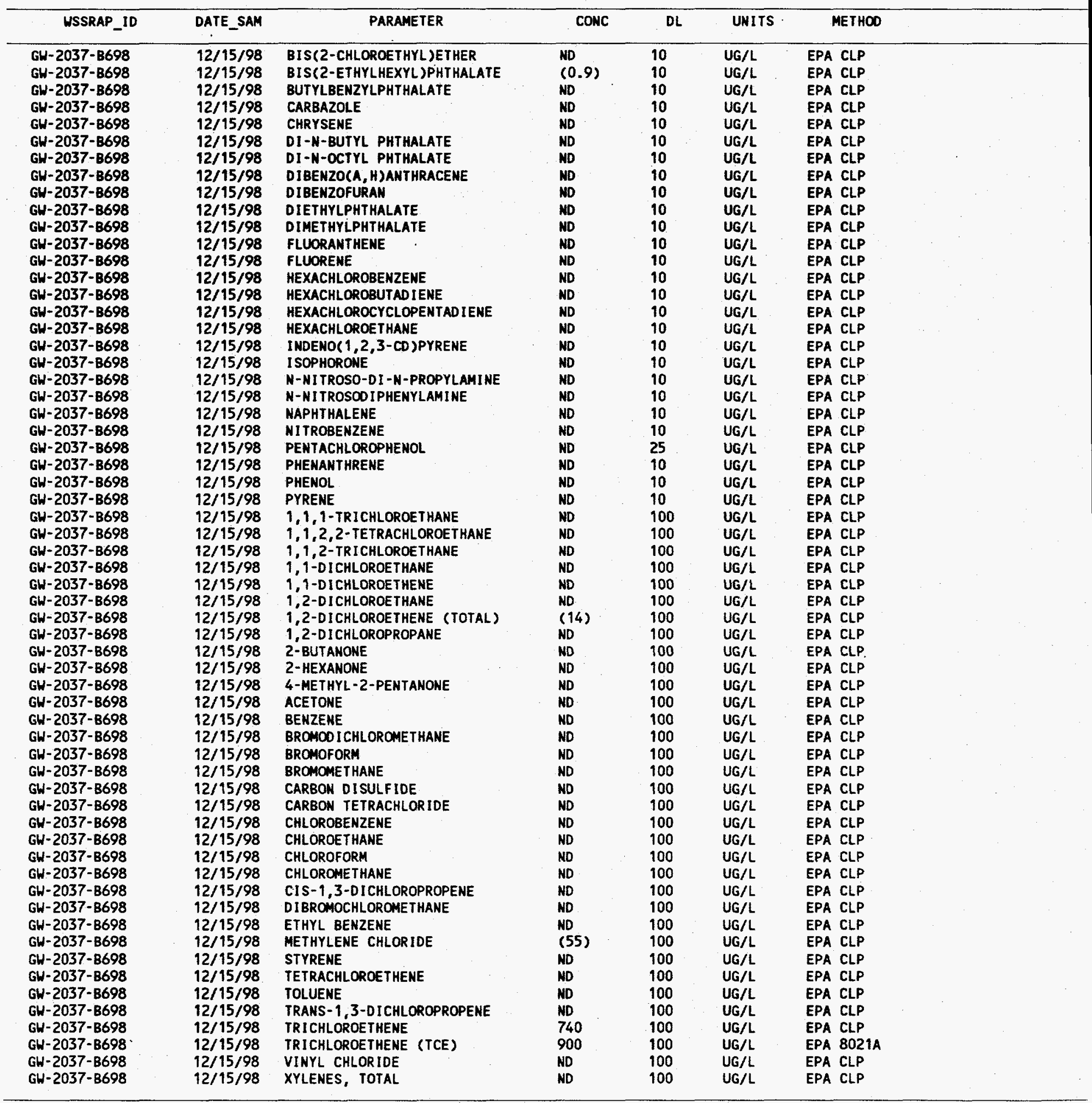




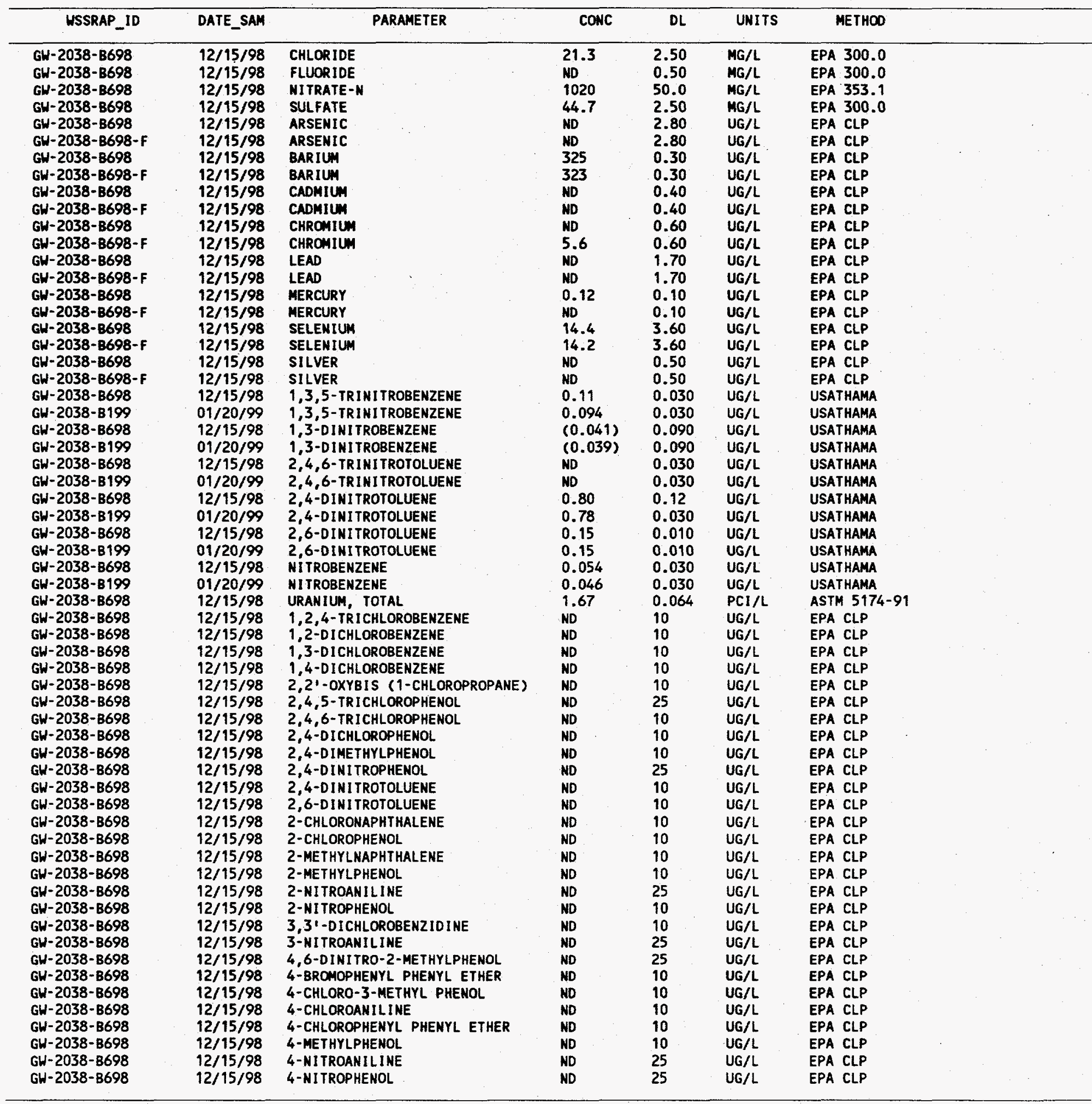




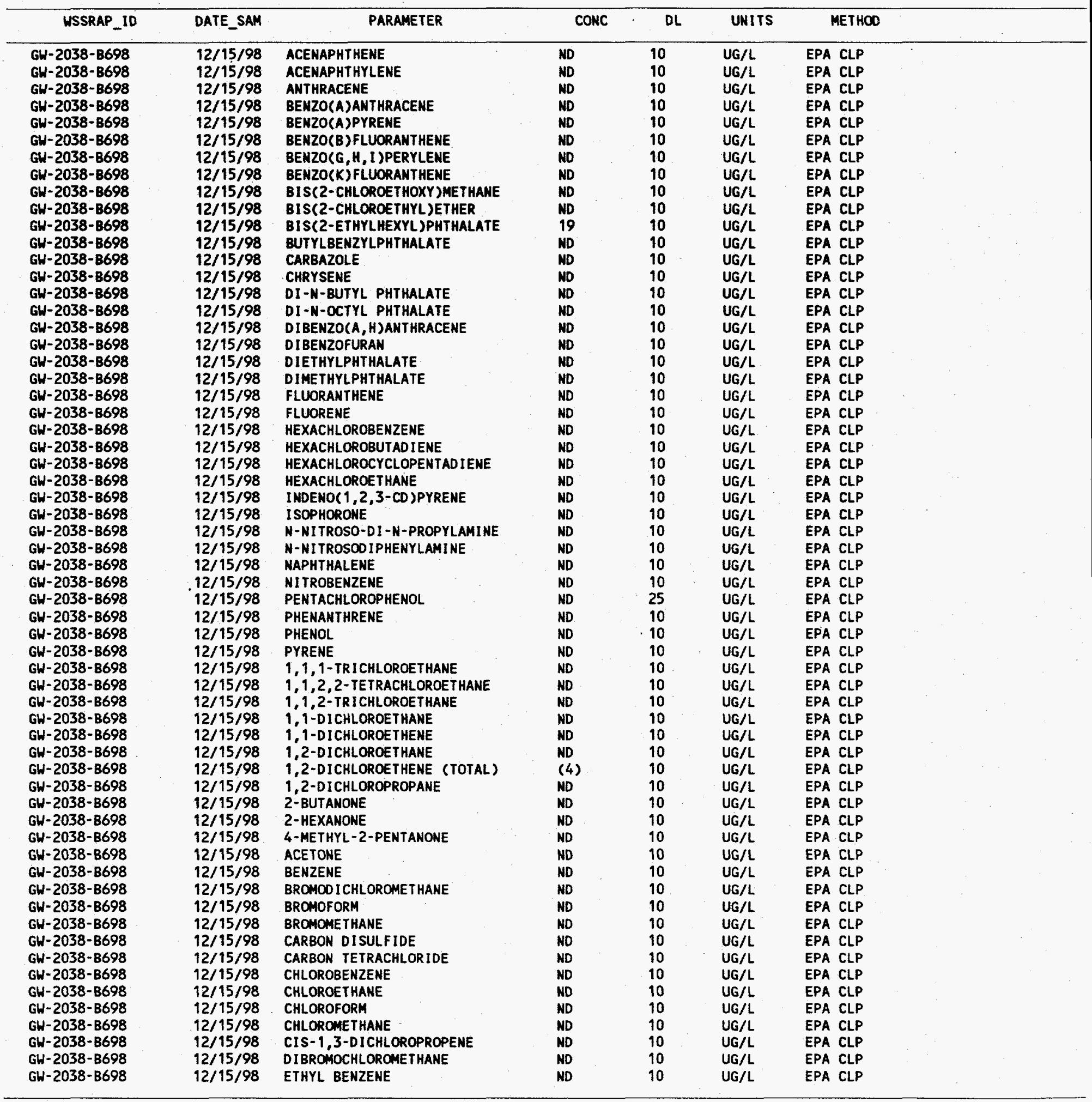




\begin{tabular}{|c|c|c|c|c|c|c|}
\hline USSRAP_ID & DATE_SAM & PARAMETER & CONC & DL & UNITS & METHOO \\
\hline 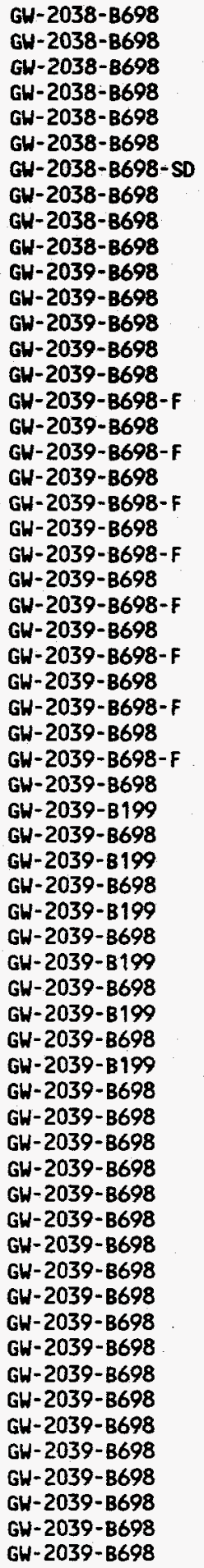 & $\begin{array}{l}12 / 15 / 98 \\
12 / 15 / 98 \\
12 / 15 / 98 \\
12 / 15 / 98 \\
12 / 15 / 98 \\
12 / 15 / 98 \\
12 / 15 / 98 \\
12 / 15 / 98 \\
12 / 15 / 98 \\
12 / 15 / 98 \\
12 / 14 / 98 \\
12 / 14 / 98 \\
12 / 14 / 98 \\
12 / 14 / 98 \\
12 / 14 / 98 \\
12 / 14 / 98 \\
12 / 14 / 98 \\
12 / 14 / 98 \\
12 / 14 / 98 \\
12 / 14 / 98 \\
12 / 14 / 98 \\
12 / 14 / 98 \\
12 / 14 / 98 \\
12 / 14 / 98 \\
12 / 14 / 98 \\
12 / 14 / 98 \\
12 / 14 / 98 \\
12 / 14 / 98 \\
12 / 14 / 98 \\
12 / 14 / 98 \\
12 / 14 / 98 \\
01 / 20 / 99 \\
12 / 14 / 98 \\
01 / 20 / 99 \\
12 / 14 / 98 \\
01 / 20 / 99 \\
12 / 14 / 98 \\
01 / 20 / 99 \\
12 / 14 / 98 \\
01 / 20 / 99 \\
12 / 14 / 98 \\
01 / 20 / 99 \\
12 / 14 / 98 \\
12 / 14 / 98 \\
12 / 14 / 98 \\
12 / 14 / 98 \\
12 / 14 / 98 \\
12 / 14 / 98 \\
12 / 14 / 98 \\
12 / 14 / 98 \\
12 / 14 / 98 \\
12 / 14 / 98 \\
12 / 14 / 98 \\
12 / 14 / 98 \\
12 / 14 / 98 \\
12 / 14 / 98 \\
12 / 14 / 98 \\
12 / 14 / 98 \\
12 / 14 / 98 \\
12 / 14 / 98 \\
12 / 14 / 98\end{array}$ & 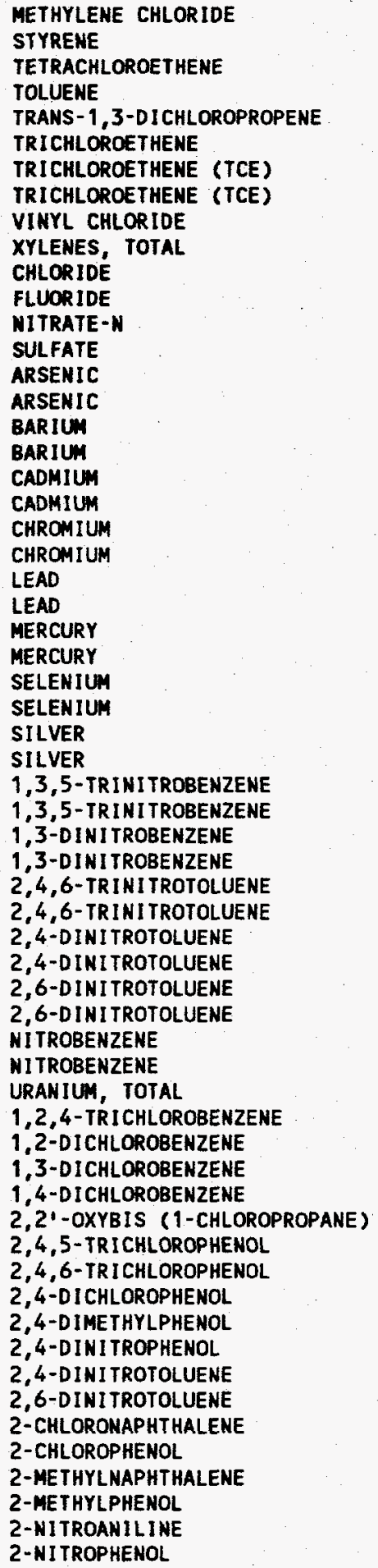 & 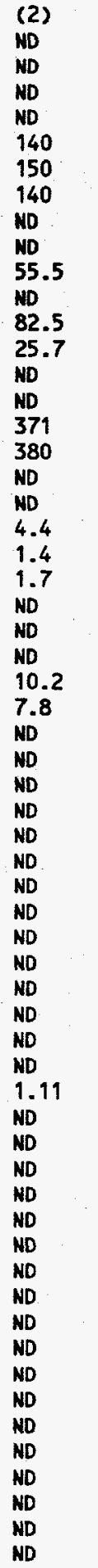 & $\begin{array}{l}10 \\
10 \\
10 \\
10 \\
10 \\
10 \\
20 \\
5.00 \\
10 \\
10 \\
2.50 \\
0.50 \\
5.00 \\
1.20 \\
2.80 \\
2.80 \\
0.30 \\
0.30 \\
0.40 \\
0.40 \\
0.60 \\
0.60 \\
1.70 \\
1.70 \\
0.10 \\
0.10 \\
3.60 \\
3.60 \\
0.50 \\
0.50 \\
0.030 \\
0.030 \\
0.090 \\
0.090 \\
0.030 \\
0.030 \\
0.030 \\
0.030 \\
0.010 \\
0.010 \\
0.030 \\
0.030 \\
0.677 \\
10 \\
10 \\
10 \\
10 \\
10 \\
25 \\
10 \\
10 \\
10 \\
25 \\
10 \\
10 \\
10 \\
10 \\
10 \\
10 \\
25 \\
10 \\
\\
10 \\
\\
\\
\\
\end{array}$ & $\begin{array}{l}U G / L \\
U G / L \\
U G / L \\
U G / L \\
U G / L \\
U G / L \\
U G / L \\
U G / L \\
U G / L \\
U G / L \\
M G / L \\
U G / L \\
U G / L \\
U G / L \\
U G / L \\
U G / L \\
U G / L \\
U G / L \\
U G / L \\
U G / L \\
U G / L \\
U G / L \\
U G / L \\
U G / L \\
U G / L \\
U G / L \\
U G / L \\
U G / L \\
U G / L \\
U G / L \\
U G / L \\
U G / L \\
U G / L \\
U G / L \\
U G / L \\
U G / L \\
U G / L \\
U G / L \\
U G / L \\
U G / L \\
U G / L \\
U G / L \\
U G / L \\
U G / L \\
U G / L \\
U G / L \\
U G / L \\
U G / L \\
U G / L \\
U G / L \\
U G / L \\
U G / L \\
U G / L \\
U G / L \\
U G \\
U G \\
U G \\
U G\end{array}$ & $\begin{array}{l}\text { EPA CLP } \\
\text { EPA CLP } \\
\text { EPA CLP } \\
\text { EPA CLP } \\
\text { EPA CLP } \\
\text { EPA CLP } \\
\text { EPA } 8260 \\
\text { EPA } 8021 A \\
\text { EPA CLP } \\
\text { EPA CLP } \\
\text { EPA } 300.0 \\
\text { EPA } 300.0 \\
\text { EPA } 353.1 \\
\text { EPA } 300.0 \\
\text { EPA CLP } \\
\text { EPA CLP } \\
\text { EPA CLP } \\
\text { EPA CLP } \\
\text { EPA CLP } \\
\text { EPA CLP } \\
\text { EPA CLP } \\
\text { EPA CLP } \\
\text { EPA CLP } \\
\text { EPA CLP } \\
\text { EPA CLP } \\
\text { EPA CLP } \\
\text { EPA CLP } \\
\text { EPA CLP } \\
\text { EPA CLP } \\
\text { EPA CLP } \\
\text { USATHAMA } \\
\text { USATHAMA } \\
\text { USATHAMA } \\
\text { USATHAMA } \\
\text { USATHAMA } \\
\text { USATHAMA } \\
\text { USATHAMA } \\
\text { USATHAMA } \\
\text { USATHAMA } \\
\text { USATHAMA } \\
\text { USATHAMA } \\
\text { USATHAMA } \\
\text { ASTM5 174M } \\
\text { EPA CLP } \\
\text { EPA CLP } \\
\text { EPA CLP } \\
\text { EPA CLP } \\
\text { EPA CLP } \\
\text { EPA CLP } \\
\text { EPA CLP } \\
\text { EPA CLP } \\
\text { EPA CLPP } \\
\text { EPA CLP } \\
\text { EPA CLP } \\
\text { EPA CLP } \\
\text { EPA CLPP } \\
\text { EPA CLP } \\
\text { EPA CLP } \\
\text { EPA CLP } \\
\text { EPA CLP } \\
\text { EPA CLP }\end{array}$ \\
\hline
\end{tabular}




\begin{tabular}{|c|c|c|c|c|c|c|}
\hline WSSRAP_ID & DATE_SAM & PARAMETER & CONC & $\mathrm{DL}$ & UNITS & METHOD \\
\hline 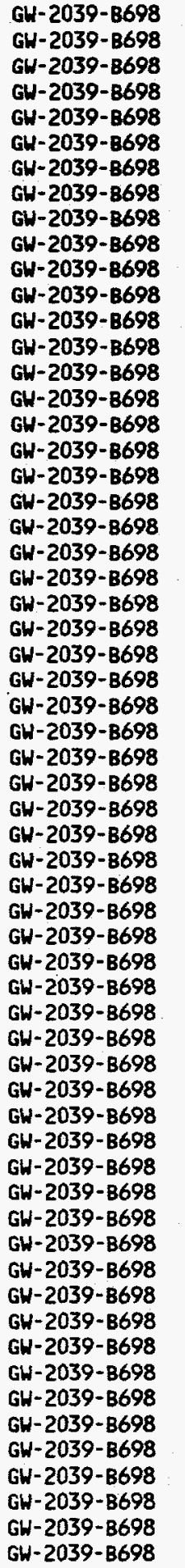 & $\begin{array}{l}12 / 14 / 98 \\
12 / 14 / 98 \\
12 / 14 / 98 \\
12 / 14 / 98 \\
12 / 14 / 98 \\
12 / 14 / 98 \\
12 / 14 / 98 \\
12 / 14 / 98 \\
12 / 14 / 98 \\
12 / 14 / 98 \\
12 / 14 / 98 \\
12 / 14 / 98 \\
12 / 14 / 98 \\
12 / 14 / 98 \\
12 / 14 / 98 \\
12 / 14 / 98 \\
12 / 14 / 98 \\
12 / 14 / 98 \\
12 / 14 / 98 \\
12 / 14 / 98 \\
12 / 14 / 98 \\
12 / 14 / 98 \\
12 / 14 / 98 \\
12 / 14 / 98 \\
12 / 14 / 98 \\
12 / 14 / 98 \\
12 / 14 / 98 \\
12 / 14 / 98 \\
12 / 14 / 98 \\
12 / 14 / 98 \\
12 / 14 / 98 \\
12 / 14 / 98 \\
12 / 14 / 98 \\
12 / 14 / 98 \\
12 / 14 / 98 \\
12 / 14 / 98 \\
12 / 14 / 98 \\
12 / 14 / 98 \\
12 / 14 / 98 \\
12 / 14 / 98 \\
12 / 14 / 98 \\
12 / 14 / 98 \\
12 / 14 / 98 \\
12 / 14 / 98 \\
12 / 14 / 98 \\
12 / 14 / 98 \\
12 / 14 / 98 \\
12 / 14 / 98 \\
12 / 14 / 98 \\
12 / 14 / 98 \\
12 / 14 / 98 \\
12 / 14 / 98 \\
12 / 14 / 98 \\
12 / 14 / 98 \\
12 / 14 / 98 \\
12 / 14 / 98 \\
12 / 14 / 98 \\
12 / 14 / 98 \\
12 / 14 / 98 \\
12 / 14 / 98 \\
12 / 14 / 98\end{array}$ & 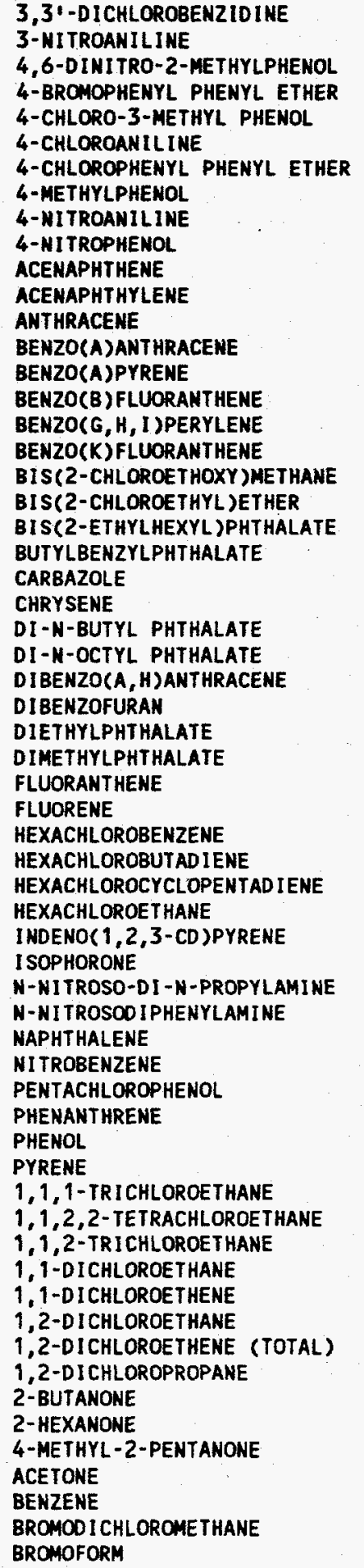 & $\begin{array}{l}\text { ND } \\
\text { ND } \\
\text { ND } \\
\text { ND } \\
\text { ND } \\
\text { ND } \\
\text { ND } \\
\text { ND } \\
\text { ND } \\
\text { ND } \\
\text { HD } \\
\text { ND } \\
\text { ND } \\
\text { ND } \\
\text { ND } \\
\text { ND } \\
\text { ND } \\
\text { ND } \\
\text { ND } \\
\text { ND } \\
\text { ND } \\
\text { ND } \\
\text { ND } \\
\text { ND } \\
\text { ND } \\
\text { ND } \\
\text { ND } \\
\text { ND } \\
\text { ND } \\
\text { ND } \\
\text { ND } \\
\text { ND } \\
\text { ND } \\
\text { ND } \\
\text { ND } \\
\text { ND } \\
\text { ND } \\
\text { ND } \\
\text { ND } \\
\text { ND } \\
\text { ND } \\
\text { ND } \\
\text { ND } \\
\text { ND } \\
\text { ND } \\
\text { ND } \\
\text { ND } \\
\text { ND } \\
\text { ND } \\
\text { ND } \\
\text { ND } \\
\text { ND } \\
\text { ND } \\
\text { ND } \\
\text { ND } \\
\text { ND } \\
\text { ND } \\
\text { ND } \\
\text { ND } \\
\text { ND } \\
\text { ND }\end{array}$ & $\begin{array}{l}10 \\
25 \\
25 \\
10 \\
10 \\
10 \\
10 \\
10 \\
25 \\
25 \\
10 \\
10 \\
10 \\
10 \\
10 \\
10 \\
10 \\
10 \\
10 \\
10 \\
10 \\
10 \\
10 \\
10 \\
10 \\
10 \\
10 \\
10 \\
10 \\
10 \\
10 \\
10 \\
10 \\
10 \\
10 \\
10 \\
10 \\
10 \\
10 \\
10 \\
10 \\
10 \\
25 \\
10 \\
10 \\
10 \\
10 \\
10 \\
10 \\
10 \\
10 \\
10 \\
10 \\
10 \\
10 \\
10 \\
10 \\
10 \\
10 \\
10 \\
10\end{array}$ & $\begin{array}{l}\text { UG/L } \\
U G / L \\
U G / L \\
U G / L \\
U G / L \\
U G / L \\
U G / L \\
U G / L \\
U G / L \\
U G / L \\
U G / L \\
U G / L \\
U G / L \\
U G / L \\
U G / L \\
U G / L \\
U G / L \\
U G / L \\
U G / L \\
U G / L \\
U G / L \\
U G / L \\
U G / L \\
U G / L \\
U G / L \\
U G / L \\
U G / L \\
U G / L \\
U G / L \\
U G / L \\
U G / L \\
U G / L \\
U G / L \\
U G / L \\
U G / L \\
U G / L \\
U G / L \\
U G / L \\
U G / L \\
U G / L \\
U G / L \\
U G / L \\
U G / L \\
U G / L \\
U G / L \\
U G / L \\
U G / L \\
U G / L \\
U G / L \\
U G / L \\
U G / L \\
U G / L \\
U G / L \\
U G / L \\
U G / L \\
U G / L \\
U G / L \\
U G / L \\
U\end{array}$ & $\begin{array}{l}\text { EPA } C L P \\
\text { EPA CLP } \\
\text { EPA CLP } \\
\text { EPA CLP } \\
\text { EPA CLP } \\
\text { EPA CLP } \\
\text { EPA CLP } \\
\text { EPA CLP } \\
\text { EPA CLP } \\
\text { EPA CLP } \\
\text { EPA CLP } \\
\text { EPA CLP } \\
\text { EPA CLP } \\
\text { EPA CLP } \\
\text { EPA CLP } \\
\text { EPA CLP } \\
\text { EPA CLP } \\
\text { EPA CLP } \\
\text { EPA CLP } \\
\text { EPA CLP } \\
\text { EPA CLP } \\
\text { EPA CLP } \\
\text { EPA CLP } \\
\text { EPA CLP } \\
\text { EPA CLP } \\
\text { EPA CLP } \\
\text { EPA CLP } \\
\text { EPA CLP } \\
\text { EPA CLP } \\
\text { EPA CLP } \\
\text { EPA CLP } \\
\text { EPA CLP } \\
\text { EPA CLP } \\
\text { EPA CLP } \\
\text { EPA CLP } \\
\text { EPA CLP } \\
\text { EPA CLP } \\
\text { EPA CLP } \\
\text { EPA CLP } \\
\text { EPA CLP } \\
\text { EPA CLP } \\
\text { EPA CLP } \\
\text { EPA CLP } \\
\text { EPA CLP } \\
\text { EPA CLP } \\
\text { EPA CLP } \\
\text { EPA CLP } \\
\text { EPA CLP } \\
\text { EPA CLP } \\
\text { EPA CLP } \\
\text { EPA CLP } \\
\text { EPA CLP } \\
\text { EPA CLP } \\
\text { EPA CLP } \\
\text { EPA CLP } \\
\text { EPA CLP } \\
\text { EPA CLP } \\
\text { EPA CLP } \\
\text { EPA CLP } \\
\text { EPA CLP } \\
\text { EPA CLP }\end{array}$ \\
\hline
\end{tabular}




\begin{tabular}{|c|c|c|c|c|c|c|}
\hline WSSRAP_ID & DATE_SAM & PARAMETER & CONC & DL & UNITS & METHOO \\
\hline 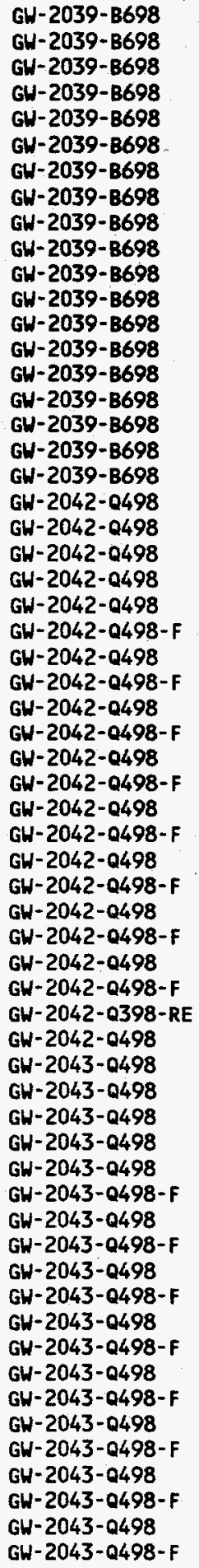 & $\begin{array}{l}12 / 14 / 98 \\
12 / 14 / 98 \\
12 / 14 / 98 \\
12 / 14 / 98 \\
12 / 14 / 98 \\
12 / 14 / 98 \\
12 / 14 / 98 \\
12 / 14 / 98 \\
12 / 14 / 98 \\
12 / 14 / 98 \\
12 / 14 / 98 \\
12 / 14 / 98 \\
12 / 14 / 98 \\
12 / 14 / 98 \\
12 / 14 / 98 \\
12 / 14 / 98 \\
12 / 14 / 98 \\
12 / 14 / 98 \\
12 / 14 / 98 \\
11 / 05 / 98 \\
11 / 05 / 98 \\
11 / 05 / 98 \\
11 / 05 / 98 \\
11 / 05 / 98 \\
11 / 05 / 98 \\
11 / 05 / 98 \\
11 / 05 / 98 \\
11105 / 98 \\
111 / 05 / 98 \\
11 / 05 / 98 \\
11 / 05 / 98 \\
11 / 05 / 98 \\
11 / 05 / 98 \\
111 / 05 / 98 \\
11 / 05 / 98 \\
11 / 05 / 98 \\
11 / 05 / 98 \\
11 / 05 / 98 \\
11 / 05 / 98 \\
08 / 06 / 98 \\
11 / 05 / 98 \\
11 / 10 / 98 \\
11 / 10 / 98 \\
11 / 10 / 98 \\
11 / 10 / 98 \\
11 / 10 / 98 \\
11 / 10 / 98 \\
11110 / 98 \\
11 / 10 / 98 \\
11 / 10 / 98 \\
11 / 10 / 98 \\
11110 / 98 \\
11110 / 98 \\
11 / 10 / 98 \\
11 / 10 / 98 \\
11 / 10 / 98 \\
11 / 10 / 98 \\
11 / 10 / 98 \\
11 / 10 / 98 \\
11 / 10 / 98 \\
11 / 10 / 98\end{array}$ & 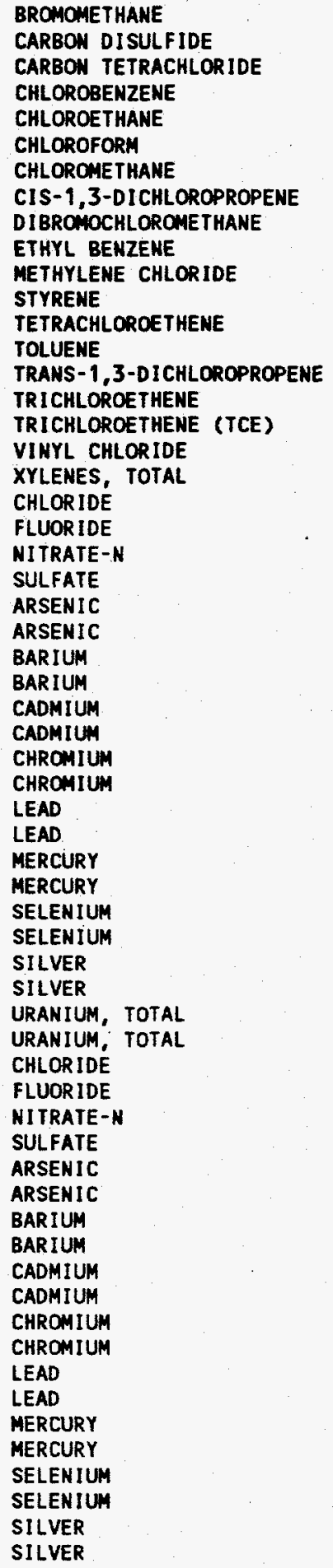 & $\begin{array}{l}\text { ND } \\
\text { ND } \\
\text { ND } \\
\text { ND } \\
\text { ND } \\
\text { ND } \\
\text { ND } \\
\text { ND } \\
\text { ND } \\
\text { ND } \\
(2) \\
\text { ND } \\
\text { ND } \\
\text { ND } \\
\text { ND } \\
\text { ND } \\
\text { ND } \\
\text { ND } \\
\text { ND } \\
42.9 \\
0.34 \\
5.96 \\
37.6 \\
2.0 \\
\text { ND } \\
414 \\
412 \\
\text { ND } \\
\text { ND } \\
7.8 \\
4.2 \\
1.2 \\
0.93 \\
\text { ND } \\
\text { ND } \\
\text { ND } \\
\text { ND } \\
\text { ND } \\
\text { ND } \\
12.4 \\
5.25 \\
5.92 \\
0.22 \\
8.05 \\
15.4 \\
\text { ND } \\
\text { ND } \\
351 \\
325 \\
\text { ND } \\
\text { ND } \\
8.3 \\
3.5 \\
6.2 \\
\text { ND } \\
\text { ND } \\
\text { ND } \\
2.7 \\
\text { ND } \\
1.5 \\
\text { ND }\end{array}$ & $\begin{array}{l}10 \\
10 \\
10 \\
10 \\
10 \\
10 \\
10 \\
10 \\
10 \\
10 \\
10 \\
10 \\
10 \\
10 \\
10 \\
10 \\
1.00 \\
10 \\
10 \\
2.00 \\
0.20 \\
0.50 \\
1.00 \\
1.8 \\
1.8 \\
0.40 \\
0.40 \\
0.40 \\
0.40 \\
0.80 \\
0.80 \\
0.90 \\
0.90 \\
0.10 \\
0.10 \\
2.2 \\
2.2 \\
1.3 \\
1.3 \\
6.77 \\
0.677 \\
0.40 \\
0.10 \\
1.25 \\
0.50 \\
1.8 \\
1.8 \\
0.40 \\
0.40 \\
0.40 \\
0.40 \\
0.80 \\
0.80 \\
0.90 \\
0.90 \\
0.10 \\
0.10 \\
2.2 \\
2.2 \\
1.3 \\
1.3 \\
\end{array}$ & 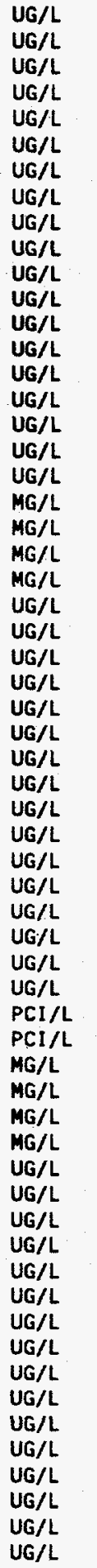 & $\begin{array}{l}\text { EPA CLP } \\
\text { EPA CLP } \\
\text { EPA CLP } \\
\text { EPA CLP } \\
\text { EPA CLP } \\
\text { EPA CLP } \\
\text { EPA CLP } \\
\text { EPA CLP } \\
\text { EPA CLP } \\
\text { EPA CLP } \\
\text { EPA CLP } \\
\text { EPA CLP } \\
\text { EPA CLP } \\
\text { EPA CLP } \\
\text { EPA CLP } \\
\text { EPA CLP } \\
\text { EPA } 8021 A \\
\text { EPA CLP } \\
\text { EPA CLP } \\
\text { EPA } 300.0 \\
\text { EPA } 300.0 \\
\text { EPA } 353.1 \\
\text { EPA } 300.0 \\
\text { EPA CLP } \\
\text { EPA CLP } \\
\text { EPA CLP } \\
\text { EPA CLP } \\
\text { EPA CLP } \\
\text { EPA CLP } \\
\text { EPA CLP } \\
\text { EPA CLP } \\
\text { EPA CLP } \\
\text { EPA CLP } \\
\text { EPA CLP } \\
\text { EPA CLP } \\
\text { EPA CLP } \\
\text { EPA CLP } \\
\text { EPA CLP } \\
\text { EPA CLP } \\
\text { ASTM } 5174-91 \\
\text { ASTM } 5174-91 \\
\text { EPA } 300.0 \\
\text { EPA } 300.0 \\
\text { EPA } 353.1 \\
\text { EPA } 300.0 \\
\text { EPA CLP } \\
\text { EPA CLP } \\
\text { EPA CLP } \\
\text { EPA CLP } \\
\text { EPA CLP } \\
\text { EPA CLP } \\
\text { EPA CLP } \\
\text { EPA CLP } \\
\text { EPA CLP } \\
\text { EPA CLP } \\
\text { EPA CLP } \\
\text { EPA CLP } \\
\text { EPA CLP } \\
\text { EPA CLP } \\
\text { EPA CLP } \\
\text { EPA CLP }\end{array}$ \\
\hline
\end{tabular}




\begin{tabular}{|c|c|c|c|c|c|c|}
\hline WSSRAP_ID & DATE_SAM & PARAMETER & CONC & DL & UNITS & METHOO \\
\hline 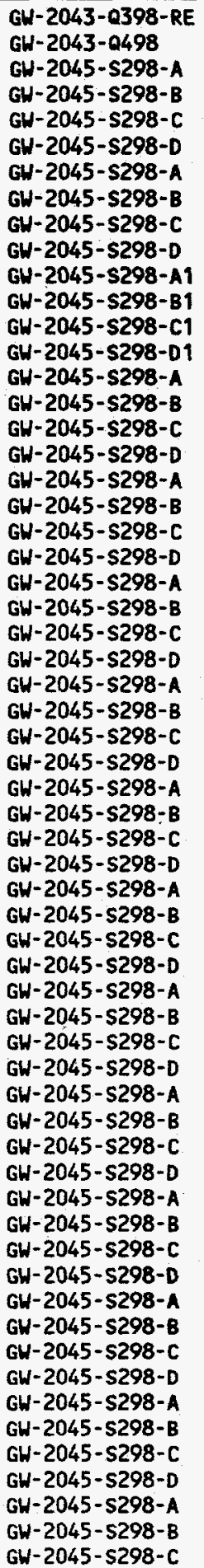 & $\begin{array}{l}08 / 06 / 98 \\
11 / 10 / 98 \\
12 / 07 / 98 \\
12 / 09 / 98 \\
12 / 11 / 98 \\
12 / 14 / 98 \\
12 / 07 / 98 \\
12 / 09 / 98 \\
12 / 11 / 98 \\
12 / 14 / 98 \\
12 / 08 / 98 \\
12 / 10 / 98 \\
12 / 12 / 98 \\
12 / 15 / 98 \\
12 / 07 / 98 \\
12 / 09 / 98 \\
12 / 11 / 98 \\
12 / 14 / 98 \\
12 / 07 / 98 \\
12 / 09 / 98 \\
12 / 11 / 98 \\
12 / 14 / 98 \\
12 / 07 / 98 \\
12 / 09 / 98 \\
12 / 11 / 98 \\
12 / 14 / 98 \\
12 / 07 / 98 \\
12 / 09 / 98 \\
12 / 11 / 98 \\
12 / 14 / 98 \\
12 / 07 / 98 \\
12 / 09 / 98 \\
12 / 11 / 98 \\
12 / 14 / 98 \\
12 / 07 / 98 \\
12 / 09 / 98 \\
12 / 11 / 98 \\
12 / 14 / 98 \\
12 / 07 / 98 \\
12 / 09 / 98 \\
12 / 11 / 98 \\
12 / 14 / 98 \\
12 / 07 / 98 \\
12 / 09 / 98 \\
12 / 11 / 98 \\
12 / 14 / 98 \\
12 / 07 / 98 \\
12 / 09 / 98 \\
12 / 11 / 98 \\
12 / 14 / 98 \\
12 / 07 / 98 \\
12 / 09 / 98 \\
12 / 11 / 98 \\
12 / 14 / 98 \\
12 / 07 / 98 \\
12 / 09 / 98 \\
12 / 11 / 98 \\
12 / 14 / 98 \\
12 / 07 / 98 \\
12 / 09 / 98 \\
12 / 11 / 98\end{array}$ & $\begin{array}{l}\text { URANIUH, TOTAL } \\
\text { URANIUH, TOTAL } \\
\text { CHLORIDE } \\
\text { CHLORIDE } \\
\text { CHLORIDE } \\
\text { CHLORIDE } \\
\text { FLUORIDE } \\
\text { FLUORIDE } \\
\text { FLUORIDE } \\
\text { FLUORIDE } \\
\text { NITRATE-N } \\
\text { NITRATE-N } \\
\text { NITRATE-N } \\
\text { HITRATE-N } \\
\text { SULFATE } \\
\text { SULFATE } \\
\text { SULFATE } \\
\text { SULFATE } \\
\text { ALUMINUM } \\
\text { ALUMINUM } \\
\text { ALUMINUM } \\
\text { ALUMINUM } \\
\text { ANTIMONY } \\
\text { ANTIMONY } \\
\text { ANTIMONY } \\
\text { ANTIMONY } \\
\text { ARSENIC } \\
\text { ARSENIC } \\
\text { ARSENIC } \\
\text { ARSENIC } \\
\text { BARIUM } \\
\text { BARIUM } \\
\text { BARIUH } \\
\text { BARIUM } \\
\text { BERYLIUM } \\
\text { BERYLLIUM } \\
\text { BERYLLIUM } \\
\text { BERYLLIUM } \\
\text { CADMIUM } \\
\text { CADMIUM } \\
\text { CADMIUM } \\
\text { CADMIUM } \\
\text { CALCIUM } \\
\text { CALCIUM } \\
\text { CALCIUM } \\
\text { CALCIIUM } \\
\text { CHROMIUM } \\
\text { CHROMIUM } \\
\text { CHROMIUM } \\
\text { CHROMIUH } \\
\text { COBALT } \\
\text { COBALT } \\
\text { COBALT } \\
\text { COBALT } \\
\text { COPPER } \\
\text { COPPERR } \\
\text { COPPER } \\
\text { COPPER } \\
\text { IRON } \\
\text { IRON } \\
\text { IRON } \\
\end{array}$ & 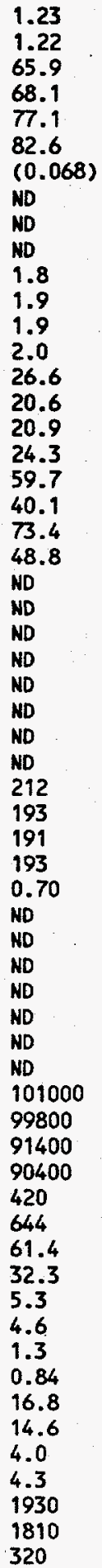 & $\begin{array}{l}0.677 \\
0.677 \\
4.00 \\
2.50 \\
2.50 \\
2.50 \\
0.10 \\
0.50 \\
0.50 \\
0.50 \\
0.10 \\
0.10 \\
0.10 \\
0.20 \\
1.00 \\
1.20 \\
1.20 \\
1.20 \\
18.7 \\
14.2 \\
14.2 \\
14.2 \\
45.9 \\
3.5 \\
3.50 \\
3.50 \\
2.5 \\
2.8 \\
2.80 \\
2.80 \\
0.70 \\
0.30 \\
0.30 \\
0.30 \\
0.50 \\
0.10 \\
0.10 \\
0.10 \\
4.2 \\
0.40 \\
0.40 \\
0.40 \\
9.9 \\
4.7 \\
4.70 \\
4.70 \\
2.8 \\
0.60 \\
0.60 \\
0.60 \\
3.4 \\
0.70 \\
0.70 \\
0.70 \\
3.0 \\
0.80 \\
0.80 \\
0.80 \\
4.6 \\
13.9 \\
13.9 \\
\\
\\
\\
\end{array}$ & $\begin{array}{l}P C I / L \\
P C I / L \\
M G / L \\
M G / L \\
M G / L \\
M G / L \\
M G / L \\
M G / L \\
M G / L \\
M G / L \\
M G / L \\
M G / L \\
M G / L \\
M G / L \\
M G / L \\
M G / L \\
M G / L \\
M G / L \\
U G / L \\
U G / L \\
U G / L \\
U G / L \\
U G / L \\
U G / L \\
U G / L \\
U G / L \\
U G / L \\
U G / L \\
U G / L \\
U G / L \\
U G / L \\
U G / L \\
U G / L \\
U G / L \\
U G / L \\
U G / L \\
U G / L \\
U G / L \\
U G / L \\
U G / L \\
U G / L \\
U G / L \\
U G / L \\
U G / L \\
U G / L \\
U G / L \\
U G / L \\
U G / L \\
U G / L \\
U G / L \\
U G / L \\
U G / L \\
U G / L \\
U G / L \\
U G / L \\
U G / L \\
U G / L \\
U G / L \\
U G / L \\
U G / L \\
U G / L\end{array}$ & $\begin{array}{l}\text { ASTM } 5174-91 \\
\text { ASTM } 5174-91 \\
\text { EPA } 300.0 \\
\text { EPA } 300.0 \\
\text { EPA } 300.0 \\
\text { EPA } 300.0 \\
\text { EPA } 300.0 \\
\text { EPA } 300.0 \\
\text { EPA } 300.0 \\
\text { EPA } 300.0 \\
\text { EPA } 353.2 \\
\text { EPA } 353.2 \\
\text { EPA } 353.1 \\
\text { EPA } 353.1 \\
\text { EPA } 300.0 \\
\text { EPA } 300.0 \\
\text { EPA } 300.0 \\
\text { EPA } 300.0 \\
\text { EPA CLP } \\
\text { EPA CLP } \\
\text { EPA CLP } \\
\text { EPA CLP } \\
\text { EPA CLP } \\
\text { EPA CLP } \\
\text { EPA CLP } \\
\text { EPA CLP } \\
\text { EPA CLP } \\
\text { EPA CLP } \\
\text { EPA CLP } \\
\text { EPA CLP } \\
\text { EPA CLP } \\
\text { EPA CLP } \\
\text { EPA CLP } \\
\text { EPA CLP } \\
\text { EPA CLP } \\
\text { EPA CLP } \\
\text { EPA CLP } \\
\text { EPA CLP } \\
\text { EPA CLP } \\
\text { EPA CLP } \\
\text { EPA CLP } \\
\text { EPA CLP } \\
\text { EPA CLP } \\
\text { EPA CLP } \\
\text { EPA CLP } \\
\text { EPA CLP } \\
\text { EPA CLP } \\
\text { EPA CLP } \\
\text { EPA CLP } \\
\text { EPA CLP } \\
\text { EPA CLP } \\
\text { EPA CLP } \\
\text { EPA CLP } \\
\text { EPA CLP } \\
\text { EPA CLP } \\
\text { EPA CLP } \\
\text { EPA CLP } \\
\text { EPA CLP } \\
\text { EPA CLP } \\
\text { EPA CLP } \\
\text { EPA CLP } \\
\end{array}$ \\
\hline
\end{tabular}




\begin{tabular}{|c|c|c|c|c|c|c|}
\hline USSRAP_ID & DATE_SAM & PARAMETER & CONC & $\mathrm{DL}$ & UNITS & METHOD \\
\hline 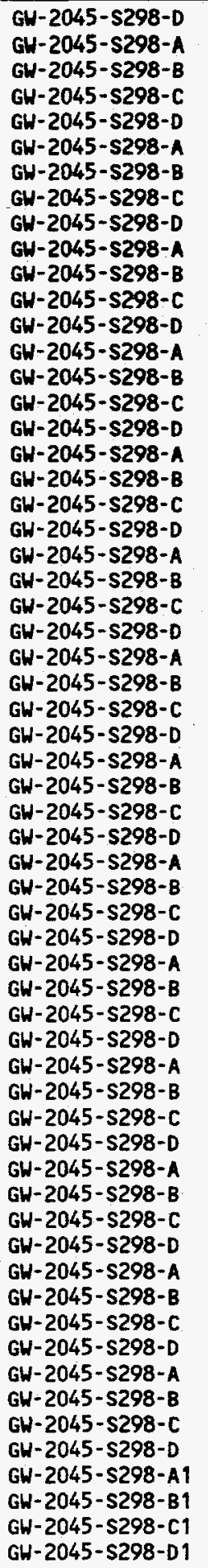 & $\begin{array}{l}12 / 14 / 98 \\
12 / 07 / 98 \\
12 / 09 / 98 \\
12 / 11 / 98 \\
12 / 14 / 98 \\
12 / 07 / 98 \\
12 / 09 / 98 \\
12 / 11 / 98 \\
12 / 14 / 98 \\
12 / 07 / 98 \\
12 / 09 / 98 \\
12 / 11 / 98 \\
12 / 14 / 98 \\
12 / 07 / 98 \\
12 / 09 / 98 \\
12 / 11 / 98 \\
12 / 14 / 98 \\
12 / 07 / 98 \\
12 / 09 / 98 \\
12 / 11 / 98 \\
12 / 14 / 98 \\
12 / 07 / 98 \\
12 / 09 / 98 \\
12 / 11 / 98 \\
12 / 14 / 98 \\
12 / 07 / 98 \\
12 / 09 / 98 \\
12 / 11 / 98 \\
12 / 14 / 98 \\
12 / 07 / 98 \\
12 / 09 / 98 \\
12 / 11 / 98 \\
12 / 14 / 98 \\
12 / 07 / 98 \\
12 / 09 / 98 \\
12 / 11 / 98 \\
12 / 14 / 98 \\
12 / 07 / 98 \\
12 / 09 / 98 \\
12 / 11 / 98 \\
12 / 14 / 98 \\
12 / 07 / 98 \\
12 / 09 / 98 \\
12 / 11 / 98 \\
12 / 14 / 98 \\
12 / 07 / 98 \\
12 / 09 / 98 \\
12 / 11 / 98 \\
12 / 14 / 98 \\
12 / 07 / 98 \\
12 / 09 / 98 \\
12 / 11 / 98 \\
12 / 14 / 98 \\
12 / 07 / 98 \\
12 / 09 / 98 \\
12 / 111 / 98 \\
12 / 14 / 98 \\
12 / 08 / 98 \\
12 / 10 / 98 \\
12 / 12 / 98 \\
12 / 15 / 98\end{array}$ & $\begin{array}{l}\text { IRON } \\
\text { LEAD } \\
\text { LEAD } \\
\text { LEAD } \\
\text { LEAD } \\
\text { LITHIUM } \\
\text { LITHIUM } \\
\text { LITHIUM } \\
\text { LITHIUM } \\
\text { MAGNESIUM } \\
\text { MAGNESIUM } \\
\text { MAGNESIUM } \\
\text { MAGNESIUM } \\
\text { MANGANESE } \\
\text { MANGANESE } \\
\text { MANGANESE } \\
\text { MANGANESE } \\
\text { MERCURY } \\
\text { MERCURY } \\
\text { MERCURY } \\
\text { MERCURY } \\
\text { MOLYBDENUM } \\
\text { MOLYBDENUM } \\
\text { MOLYBDENUM } \\
\text { MOLYBDENUM } \\
\text { NICKEL } \\
\text { NICKEL } \\
\text { NICKEL } \\
\text { NICKEL } \\
\text { POTASSIUM } \\
\text { POIASSIUM } \\
\text { POTASSIUM } \\
\text { POTASSIUM } \\
\text { SELENIUM } \\
\text { SELENIUM } \\
\text { SELENIUM } \\
\text { SELENIUM } \\
\text { SILVER } \\
\text { SILVER } \\
\text { SILVER } \\
\text { SILVER } \\
\text { SOOIUM } \\
\text { SODIUM } \\
\text { SODIUM } \\
\text { SOOIUM } \\
\text { THALLIUM } \\
\text { THALLIUM } \\
\text { THALLIUM } \\
\text { THALLIUM } \\
\text { VANADIUM } \\
\text { VANADIUM } \\
\text { VANADIUM } \\
\text { VANADIUM } \\
\text { ZINC } \\
\text { ZINC } \\
\text { ZINC } \\
\text { ZINC } \\
\text { CHEMICAL OXYGEN DEMAND } \\
\text { CHEMICAL OXYGEN DEMAND } \\
\text { CHEMICAL OXYGEN DEMAND } \\
\text { CHEMICAL OXYGEN DEMAND }\end{array}$ & $\begin{array}{l}145 \\
\text { ND } \\
\text { ND } \\
\text { ND } \\
\text { ND } \\
18.6 \\
3.4 \\
3.2 \\
3.3 \\
48100 \\
47400 \\
44600 \\
44500 \\
62.6 \\
55.0 \\
20.2 \\
9.8 \\
\text { ND } \\
\text { ND } \\
\text { ND } \\
\text { ND } \\
48.3 \\
73.2 \\
12.2 \\
9.1 \\
829 \\
627 \\
276 \\
238 \\
2240 \\
2340 \\
2340 \\
2350 \\
\text { ND } \\
\text { ND } \\
4.5 \\
\text { ND } \\
\text { ND } \\
\text { ND } \\
\text { ND } \\
\text { ND } \\
14600 \\
14300 \\
15400 \\
15000 \\
4.6 \\
\text { ND } \\
\text { ND } \\
3.7 \\
20.2 \\
4.7 \\
\text { ND } \\
\text { ND } \\
13.6 \\
23.4 \\
8.8 \\
3.9 \\
\text { ND } \\
\text { ND } \\
\end{array}$ & $\begin{array}{l}13.9 \\
1.6 \\
1.7 \\
1.70 \\
1.70 \\
7.8 \\
0.20 \\
0.20 \\
0.20 \\
62.0 \\
3.9 \\
3.90 \\
3.90 \\
0.80 \\
0.10 \\
0.10 \\
0.10 \\
0.10 \\
0.10 \\
0.10 \\
0.10 \\
11.3 \\
0.90 \\
0.90 \\
0.90 \\
15.8 \\
1.5 \\
1.50 \\
1.50 \\
2200 \\
18.2 \\
18.2 \\
18.2 \\
2.9 \\
3.6 \\
3.60 \\
3.60 \\
5.5 \\
0.50 \\
0.50 \\
0.50 \\
44.5 \\
1.4 \\
1.40 \\
1.40 \\
2.7 \\
2.8 \\
2.80 \\
2.80 \\
3.3 \\
0.60 \\
0.60 \\
0.60 \\
2.3 \\
0.60 \\
0.60 \\
0.60 \\
5.00 \\
5.00 \\
5.00 \\
5.00\end{array}$ & 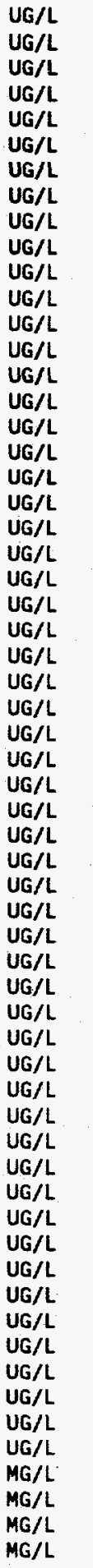 & $\begin{array}{l}\text { EPA CLP } \\
\text { EPA CLP } \\
\text { EPA CLP } \\
\text { EPA CLP } \\
\text { EPA CLP } \\
\text { EPA CLP } \\
\text { EPA CLP } \\
\text { EPA CLP } \\
\text { EPA CLP } \\
\text { EPA CLP } \\
\text { EPA CLP } \\
\text { EPA CLP } \\
\text { EPA CLP } \\
\text { EPA CLP } \\
\text { EPA CLP } \\
\text { EPA CLP } \\
\text { EPA CLP } \\
\text { EPA CLP } \\
\text { EPA CLP } \\
\text { EPA CLLP } \\
\text { EPA CLP } \\
\text { EPA CLP } \\
\text { EPA CLP } \\
\text { EPA CLP } \\
\text { EPA CLP } \\
\text { EPA CLP } \\
\text { EPA CLP } \\
\text { EPA CLP } \\
\text { EPA CLP } \\
\text { EPA CLP } \\
\text { EPA CLP } \\
\text { EPA CLP } \\
\text { EPA CLP } \\
\text { EPA CLP } \\
\text { EPA CLP } \\
\text { EPA CLP } \\
\text { EPA CLP } \\
\text { EPA CLP } \\
\text { EPA CLP } \\
\text { EPA CLP } \\
\text { EPA CLP } \\
\text { EPA CLP } \\
\text { EPA CLP } \\
\text { EPA CLP } \\
\text { EPA CLP } \\
\text { EPA CLP } \\
\text { EPA CLP } \\
\text { EPA CLP } \\
\text { EPA CLP } \\
\text { EPA CLP } \\
\text { EPA CLP } \\
\text { EPA CLP } \\
\text { EPA CLP } \\
\text { EPA CLP } \\
\text { EPA CLP } \\
\text { EPA CLP } \\
\text { EPA CLP } \\
\text { EPA } 410.0 \\
\text { EPA } 410.0 \\
\text { EPA } 410.0 \\
\text { EPA } 410.0\end{array}$ \\
\hline
\end{tabular}




\begin{tabular}{|c|c|c|c|c|c|c|c|}
\hline WSSRAP_ID & DATE_SAM & PARAMETER & conc & $\mathrm{DL}$ & UNITS & METHOD & \\
\hline $\begin{array}{l}G W-2045-S 298-A \\
G W-2045-S 298-B \\
G W-2045-S 298-C \\
G W-2045-S 298-D \\
G W-2045-S 298-A 1 \\
G W-2045-S 298-B 1 \\
G W-2045-S 298-C 1 \\
G W-2045-S 298-D 1 \\
G W-2045-S 298-A 1 \\
G W-2045-S 298-B 1 \\
G W-2045-S 298-C 1 \\
G W-2045-S 298-D 1 \\
G W-2045-S 298-A 1 \\
G W-2045-S 298-B 1 \\
G W-2045-S 298-C 1 \\
G W-2045-S 298-D 1 \\
G W-2045-S 298-A 1 \\
G W-2045-S 298-B 1 \\
G W-2045-S 298-C 1 \\
G W-2045-S 298-D 1 \\
G W-2045-S 298-A 1 \\
G W-2045-S 298-B 1 \\
G W-2045-S 298-C 1 \\
G W-2045-S 298-D 1 \\
G W-2045-S 298-A 1 \\
G W-2045-S 298-B 1 \\
G W-2045-S 298-C 1 \\
G W-2045-S 298-D 1 \\
G W-2045-S 298-A 1 \\
G W-2045-S 298-B 1 \\
G W-2045-S 298-C 1 \\
G W-2045-S 298-D 1 \\
G W-2045-S 298-A 1 \\
G W-2045-S 298-B 1 \\
G W-2045-S 298-C 1 \\
G W-2045-S 298-D 1 \\
G W-2045-S 298-A 1 \\
G W-2045-S 298-B 1 \\
G W-2045-S 298-C 1 \\
G W-2045-S 298-D 1 \\
G W-2045-S 298-A 1 \\
G W-2045-S 298-B 1 \\
G W-2045-S 298-C 1 \\
G W-2045-S 298-D 1 \\
G W-2045-S 298-A 1 \\
G W-2045-S 298-B 1 \\
G W-2045-S 298-C 1 \\
G W-2045-S 298-D 1 \\
G W-2045-S 298-A 1 \\
G W-2045-\$ 298-B 1 \\
G W-2045-S 298-C 1 \\
G W-2045-S 298-D 1 \\
G W-2045-S 298-A 1 \\
G W-2045-S 298-B 1 \\
G W-2045-S 298-C 1 \\
G W-2045-S 298-D 1 \\
G W-2045-S 298-A 1 \\
G W-2045-S 298-B 1 \\
G W-2045-S 298-C 1 \\
G W-2045-S 298-D 1 \\
G W-2045-S 298-A 1\end{array}$ & $\begin{array}{l}12 / 07 / 98 \\
12 / 09 / 98 \\
12 / 11 / 98 \\
12 / 14 / 98 \\
12 / 08 / 98 \\
12 / 10 / 98 \\
12 / 12 / 98 \\
12 / 15 / 98 \\
12 / 08 / 98 \\
12 / 10 / 98 \\
12 / 12 / 98 \\
12 / 15 / 98 \\
12 / 08 / 98 \\
12 / 10 / 98 \\
12 / 12 / 98 \\
12 / 15 / 98 \\
12 / 08 / 98 \\
12 / 10 / 98 \\
12 / 12 / 98 \\
12 / 15 / 98 \\
12 / 08 / 98 \\
12 / 10 / 98 \\
12 / 12 / 98 \\
12 / 15 / 98 \\
12 / 08 / 98 \\
12 / 10 / 98 \\
12 / 12 / 98 \\
12 / 15 / 98 \\
12 / 08 / 98 \\
12 / 10 / 98 \\
12 / 12 / 98 \\
12 / 15 / 98 \\
12 / 08 / 98 \\
12 / 10 / 98 \\
12 / 12 / 98 \\
12 / 15 / 98 \\
12 / 08 / 98 \\
12 / 10 / 98 \\
12 / 12 / 98 \\
12 / 15 / 98 \\
12 / 08 / 98 \\
12 / 10 / 98 \\
12 / 12 / 98 \\
12 / 15 / 98 \\
12 / 08 / 98 \\
12 / 10 / 98 \\
12 / 12 / 98 \\
12 / 15 / 98 \\
12 / 08 / 98 \\
12 / 10 / 98 \\
12 / 12 / 98 \\
12 / 15 / 98 \\
12 / 08 / 98 \\
12 / 10 / 98 \\
12 / 12 / 98 \\
12 / 15 / 98 \\
12 / 08 / 98 \\
12 / 10 / 98 \\
12 / 12 / 98 \\
12 / 15 / 98 \\
12 / 08 / 98\end{array}$ & 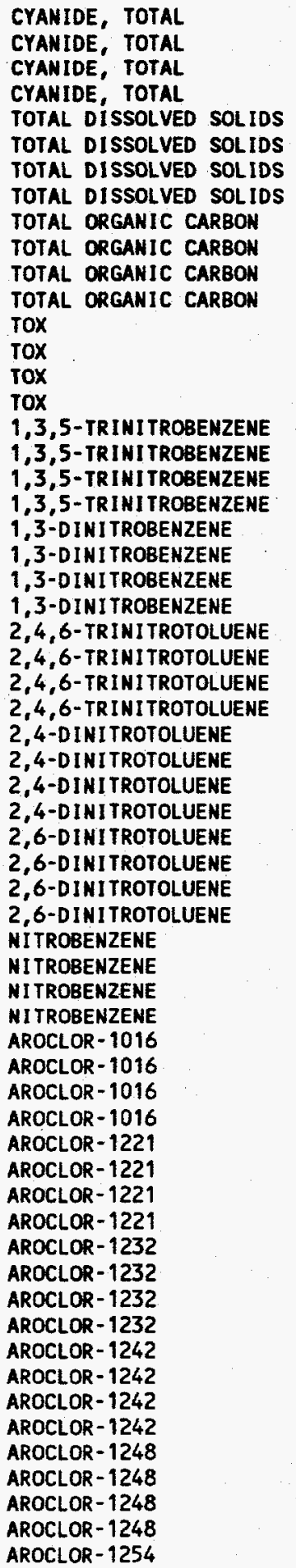 & 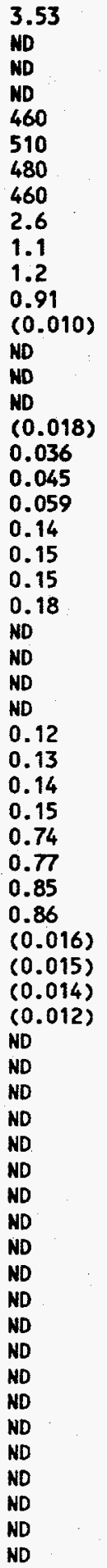 & $\begin{array}{l}1.30 \\
5.00 \\
5.00 \\
5.00 \\
5.00 \\
5.00 \\
5.00 \\
5.00 \\
0.50 \\
0.50 \\
0.50 \\
0.50 \\
0.012 \\
0.012 \\
0.012 \\
0.012 \\
0.030 \\
0.030 \\
0.030 \\
0.030 \\
0.090 \\
0.090 \\
0.090 \\
0.090 \\
0.030 \\
0.030 \\
0.030 \\
0.030 \\
0.030 \\
0.030 \\
0.030 \\
0.030 \\
0.010 \\
0.010 \\
0.010 \\
0.010 \\
0.030 \\
0.030 \\
0.030 \\
0.030 \\
1.00 \\
1.00 \\
1.00 \\
1.00 \\
2.00 \\
2.00 \\
2.00 \\
2.00 \\
1.00 \\
1.00 \\
1.00 \\
1.00 \\
1.00 \\
1.00 \\
1.00 \\
1.00 \\
1.00 \\
1.00 \\
1.00 \\
1.00 \\
1.00\end{array}$ & 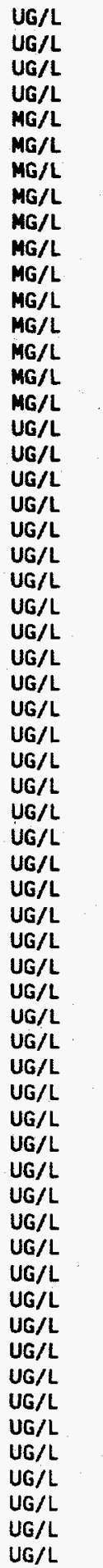 & $\begin{array}{l}\text { EPA CLP } \\
\text { EPA CLP } \\
\text { EPA CLP } \\
\text { EPA CLP } \\
\text { EPA } 160.1 \\
\text { EPA } 160.1 \\
\text { EPA } 160.1 \\
\text { EPA } 160.1 \\
\text { EPA } 415.1 \\
\text { EPA } 415.1 \\
\text { EPA } 9060 \\
\text { EPA } 415 \\
\text { EPA } 9020 \\
\text { EPA } 9020 \\
\text { EPA } 9020 \\
\text { EPA } 9020 \\
\text { USATHAMA } \\
\text { USATHAMA } \\
\text { USATHAMA } \\
\text { USATHAMA } \\
\text { USATHAMA } \\
\text { USATHAMA } \\
\text { USATHAMA } \\
\text { USATHAMA } \\
\text { USATHAMA } \\
\text { USATHAMA } \\
\text { USATHAMA } \\
\text { USATHAMA } \\
\text { USATHAMA } \\
\text { USATHAMA } \\
\text { USATHAMA } \\
\text { USATHAMA } \\
\text { USATHAMA } \\
\text { USATHAMA } \\
\text { USATHAMA } \\
\text { USATHAMA } \\
\text { USATHAMA } \\
\text { USATHAMA } \\
\text { USATHAMA } \\
\text { USATHAMA } \\
\text { EPA } 8081 \\
\text { EPA } 8081 \\
\text { EPA } 8081 \\
\text { EPA } 8081 \\
\text { EPA } 8081 \\
\text { EPA } 8081 \\
\text { EPA } 8081 \\
\text { EPA } 8081 \\
\text { EPA } 8081 \\
\text { EPA } 8081 \\
\text { EPA } 8081 \\
\text { EPA } 8081 \\
\text { EPA } 8081 \\
\text { EPA } 8081 \\
\text { EPA } 8081 \\
\text { EPA } 8081 \\
\text { EPA } 8081 \\
\text { EPA } 8081 \\
\text { EPA } 8081 \\
\text { EPA } 8081 \\
\end{array}$ & 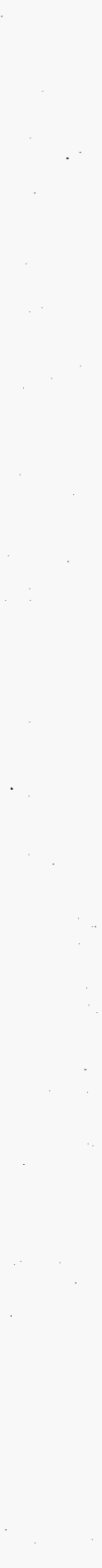 \\
\hline
\end{tabular}




\begin{tabular}{|c|c|c|c|c|c|c|}
\hline WSSRAP_ID & DATE_SAM & PARAMETER & CONC & DL & UNITS & METHOD \\
\hline 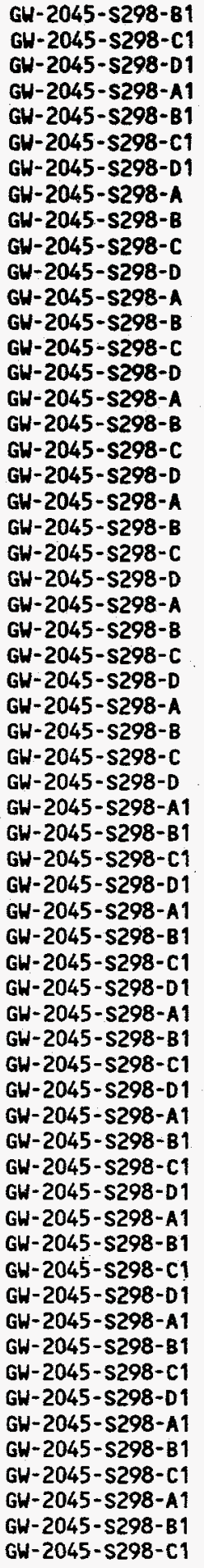 & $\begin{array}{l}12 / 10 / 98 \\
12 / 12 / 98 \\
12 / 15 / 98 \\
12 / 08 / 98 \\
12 / 10 / 98 \\
12 / 12 / 98 \\
12 / 15 / 98 \\
12 / 07 / 98 \\
12 / 09 / 98 \\
12 / 11 / 98 \\
12 / 14 / 98 \\
12 / 07 / 98 \\
12 / 09 / 98 \\
12 / 11 / 98 \\
12 / 14 / 98 \\
12 / 07 / 98 \\
12 / 09 / 98 \\
12 / 11 / 98 \\
12 / 14 / 98 \\
12 / 07 / 98 \\
12 / 09 / 98 \\
12 / 11 / 98 \\
12 / 14 / 98 \\
12 / 07 / 98 \\
12 / 09 / 98 \\
12 / 11 / 98 \\
12 / 14 / 98 \\
12 / 07 / 98 \\
12 / 09 / 98 \\
12 / 11 / 98 \\
12 / 14 / 98 \\
12 / 08 / 98 \\
12 / 10 / 98 \\
12 / 12 / 98 \\
12 / 15 / 98 \\
12 / 08 / 98 \\
12 / 10 / 98 \\
12 / 12 / 98 \\
12 / 15 / 98 \\
12 / 08 / 98 \\
12 / 10 / 98 \\
12 / 12 / 98 \\
12 / 15 / 98 \\
12 / 08 / 98 \\
12 / 10 / 98 \\
12 / 12 / 98 \\
12 / 15 / 98 \\
12 / 08 / 98 \\
12 / 10 / 98 \\
12 / 12 / 98 \\
12 / 15 / 98 \\
12 / 08 / 98 \\
12 / 10 / 98 \\
12 / 12 / 98 \\
12 / 15 / 98 \\
12 / 08 / 98 \\
12 / 10 / 98 \\
12 / 12 / 98 \\
12 / 08 / 98 \\
12 / 10 / 98 \\
12 / 12 / 98\end{array}$ & $\begin{array}{l}\text { AROCLOR-1254 } \\
\text { AROCLOR-1254 } \\
\text { AROCLOR-1254 } \\
\text { AROCLOR-1260 } \\
\text { AROCLOR-1260 } \\
\text { AROCLOR-1260 } \\
\text { AROCLOR-1260 } \\
\text { RADIUN-226 } \\
\text { RADIUH-226 } \\
\text { RADIUH-226 } \\
\text { RADIUM-226 } \\
\text { RADIUM-228 } \\
\text { RADIUM-228 } \\
\text { RADIUM-228 } \\
\text { RADIUM-228 } \\
\text { THORIUM-228 } \\
\text { THORIUM-228 } \\
\text { THORIUH-228 } \\
\text { THORIUH-228 } \\
\text { THORIUH-230 } \\
\text { THORIUM-230 } \\
\text { THORIUM-230 } \\
\text { THORIUM-230 } \\
\text { THORIUH-232 } \\
\text { THORIUM-232 } \\
\text { THORIUH-232 } \\
\text { THORIUM-232 } \\
\text { URANIUM, TOTAL } \\
\text { URANIUM, TOTAL } \\
\text { URANIUM, TOTAL } \\
\text { URANIUM, TOTAL } \\
\text { ACENAPHTHENE } \\
\text { ACENAPHTHENE } \\
\text { ACENAPHTHENE } \\
\text { ACENAPHTHENE } \\
\text { ACENAPHTHYLENE } \\
\text { ACENAPHTHYLNE } \\
\text { ACENAPHTHYLENE } \\
\text { ACENAPHTHYLENE } \\
\text { ANTHRACENE } \\
\text { ANTHRACENE } \\
\text { ANTHRACENE } \\
\text { ANTHRACENE } \\
\text { BENZO(A)ANTHRACENE } \\
\text { BENZO(A)ANTHRACENE } \\
\text { BENZO(A)ANTHRACENE } \\
\text { BENZO(A)ANTHRACENE } \\
\text { BENZO(A)PYRENE } \\
\text { BENZO(A)PYRENE } \\
\text { BENZO(A)PYRENE } \\
\text { BENZO(A)PYRENE } \\
\text { BENZO(B)FLUORANTHENE } \\
\text { BENZO(B)FLUORANTHENE } \\
\text { BENZO(B)FLUORANTHENE } \\
\text { BENZO(B)FLUORANTHENE } \\
\text { BENZO(G,H, I)PERYLENE } \\
\text { BENZO(G,H, I)PERYLENE } \\
\text { BENZO(G,H, I)PERYLENE } \\
\text { BENZO(K)FIUORANTHENE } \\
\text { BENZO(K)FLUORANTHENE } \\
\text { BENZO(K)FLUORANTHENE }\end{array}$ & 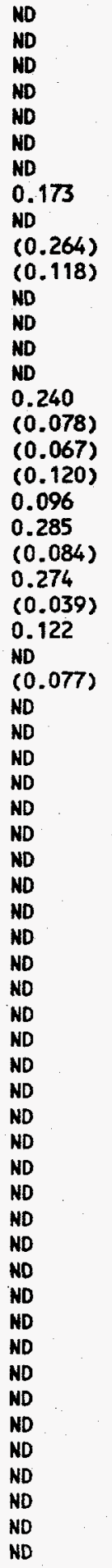 & $\begin{array}{l}1.00 \\
1.00 \\
1.00 \\
1.00 \\
1.00 \\
1.00 \\
1.00 \\
0.115 \\
0.358 \\
0.402 \\
0.326 \\
0.469 \\
0.469 \\
0.469 \\
0.469 \\
0.123 \\
0.114 \\
0.093 \\
0.144 \\
0.050 \\
0.057 \\
0.268 \\
0.119 \\
0.065 \\
0.057 \\
0.219 \\
0.175 \\
0.677 \\
0.677 \\
0.677 \\
0.677 \\
18 \\
18.0 \\
18.0 \\
18 \\
23 \\
23.0 \\
23.0 \\
23 \\
6.6 \\
6.60 \\
6.60 \\
6.60 \\
0.13 \\
0.13 \\
0.13 \\
0.13 \\
0.23 \\
0.23 \\
0.23 \\
0.23 \\
0.18 \\
0.18 \\
0.18 \\
0.18 \\
0.76 \\
0.76 \\
0.76 \\
0.17 \\
0.17 \\
0.17\end{array}$ & $\begin{array}{l}U G / L \\
U G / L \\
U G / L \\
U G / L \\
U G / L \\
U G / L \\
U G / L \\
P C I / L \\
P C I / L \\
P C I / L \\
P C I / L \\
P C I / L \\
P C I / L \\
P C I / L \\
P C I / L \\
P C I / L \\
P C I / L \\
P C I / L \\
P C I / L \\
P C I / L \\
P C I / L \\
P C I / L \\
P C I / L \\
P C I / L \\
P C I / L \\
P C I / L \\
P C I / L \\
P C I / L \\
P C I / L \\
P C I / L \\
P C I / L \\
U G / L \\
U G / L \\
U G / L \\
U G / L \\
U G / L \\
U G / L \\
U G / L \\
U G / L \\
U G / L \\
U G / L \\
U G / L \\
U G / L \\
U G / L \\
U G / L \\
U G / L \\
U G / L \\
U G / L \\
U G / L \\
U G / L \\
U G / L \\
U G / L \\
U G / L \\
U G / L \\
U G / L \\
U G / L \\
U G / L \\
U G / L \\
U G / L \\
U G / L \\
U G / L \\
\end{array}$ & $\begin{array}{l}\text { EPA } 8081 \\
\text { EPA } 8081 \\
\text { EPA } 8081 \\
\text { EPA } 8081 \\
\text { EPA } 8081 \\
\text { EPA } 8081 \\
\text { EPA } 8081 \\
\text { SM-705M } \\
\text { SM-705M } \\
\text { SM-705M } \\
\text { SM-705M } \\
\text { EPA } 9320 \\
\text { EPA } 9320 \\
\text { EPA } 9320 \\
\text { EPA } 9320 \\
\text { LANL ER200 } \\
\text { LANL ER200 } \\
\text { LANL ER200 } \\
\text { LANL ER200 } \\
\text { LANL ER200 } \\
\text { LANL ER200 } \\
\text { LANL ER200 } \\
\text { LANL ER200 } \\
\text { LANL ER200 } \\
\text { LANL ER200 } \\
\text { LANL ER200 } \\
\text { LANL ER200 } \\
\text { ASTM } 5174-91 \\
\text { ASTM } 5174-91 \\
\text { ASTM5174M } \\
\text { ASTM5174M } \\
\text { EPA } 8310 \\
\text { EPA } 8310 \\
\text { EPA } 8310 \\
\text { EPA } 8310 \\
\text { EPA } 8310 \\
\text { EPA } 8310 \\
\text { EPA } 8310 \\
\text { EPA } 8310 \\
\text { EPA } 8310 \\
\text { EPA } 8310 \\
\text { EPA } 8310 \\
\text { EPA } 8310 \\
\text { EPA } 8310 \\
\text { EPA } 8310 \\
\text { EPA } 8310 \\
\text { EPA } 8310 \\
\text { EPA } 8310 \\
\text { EPA } 8310 \\
\text { EPA } 8310 \\
\text { EPA } 8310 \\
\text { EPA } 8310 \\
\text { EPA } 8310 \\
\text { EPA } 8310 \\
\text { EPA } 8310 \\
\text { EPA } 8310 \\
\text { EPA } 8310 \\
\text { EPA } 8310 \\
\text { EPA } 8310 \\
\text { EPA } 8310 \\
\text { EPA } 8310 \\
\end{array}$ \\
\hline
\end{tabular}




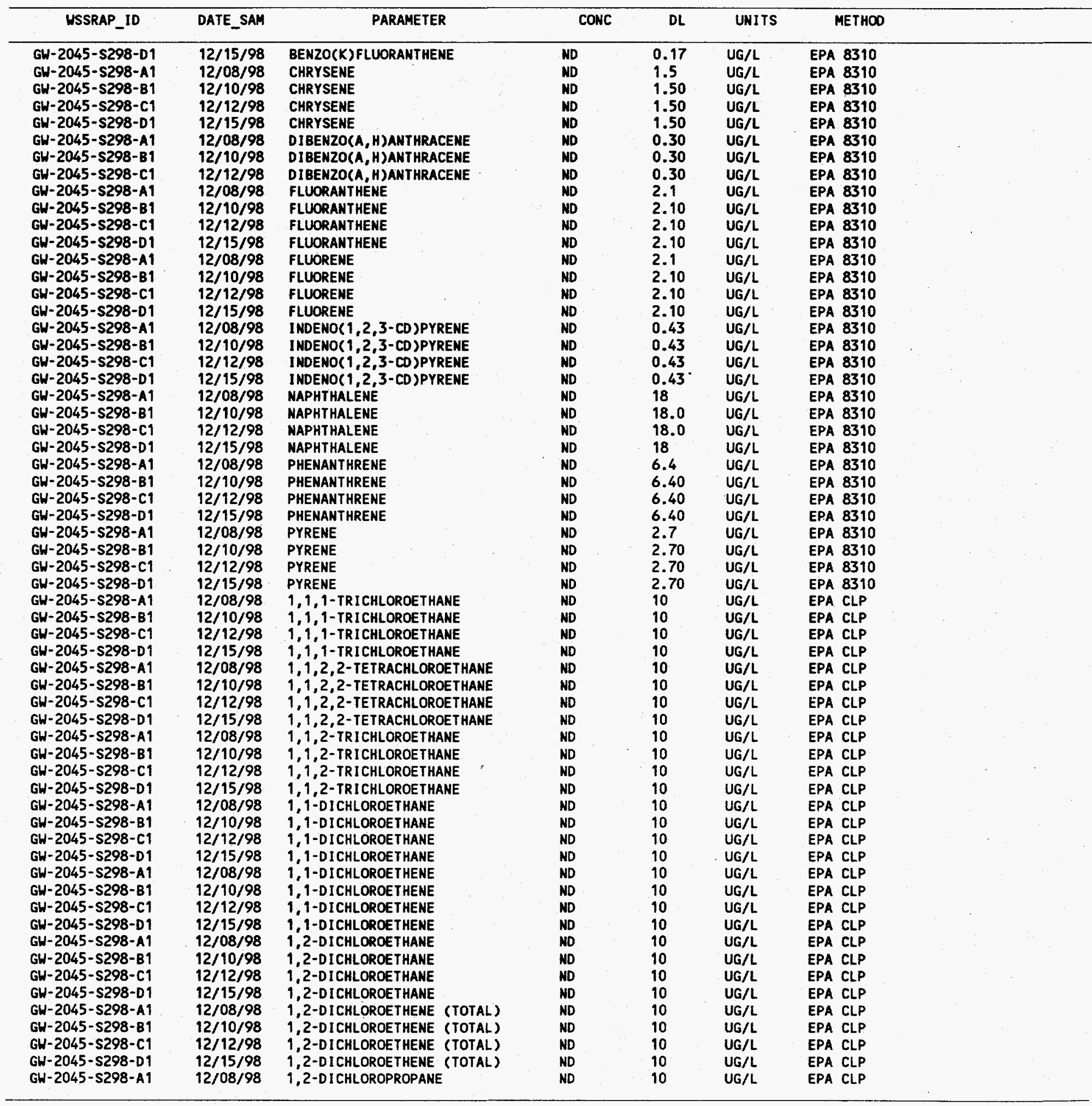




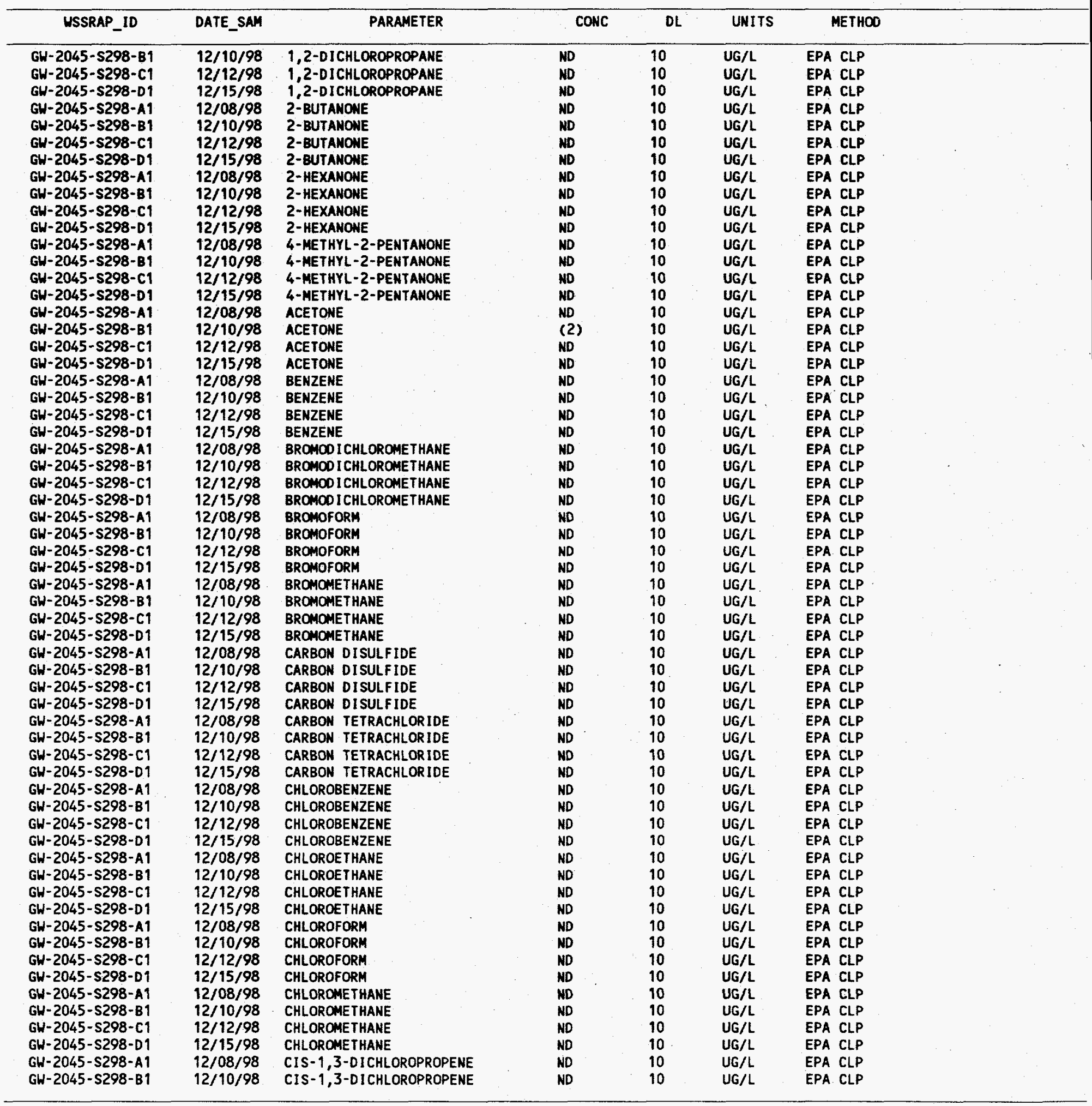




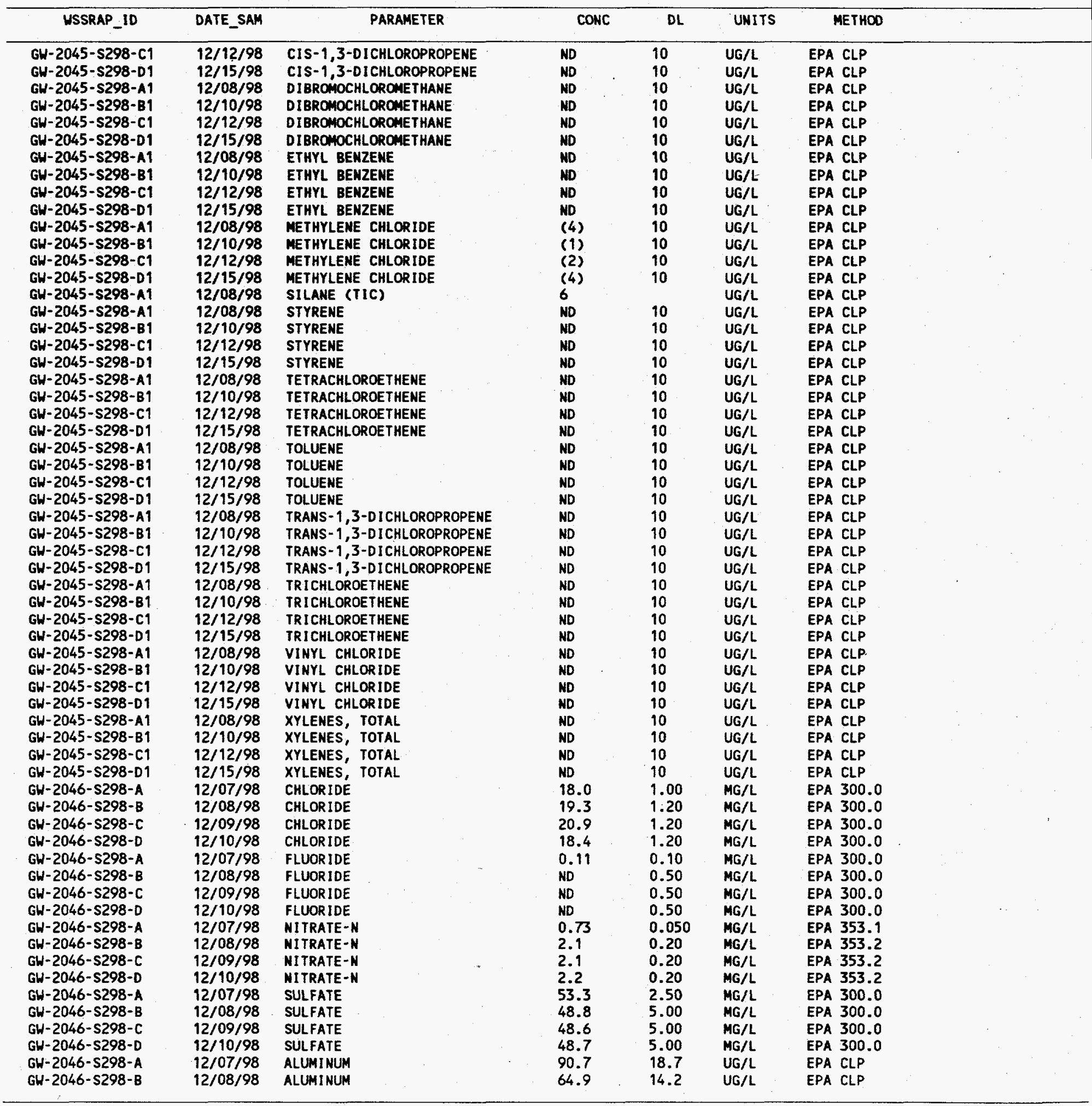




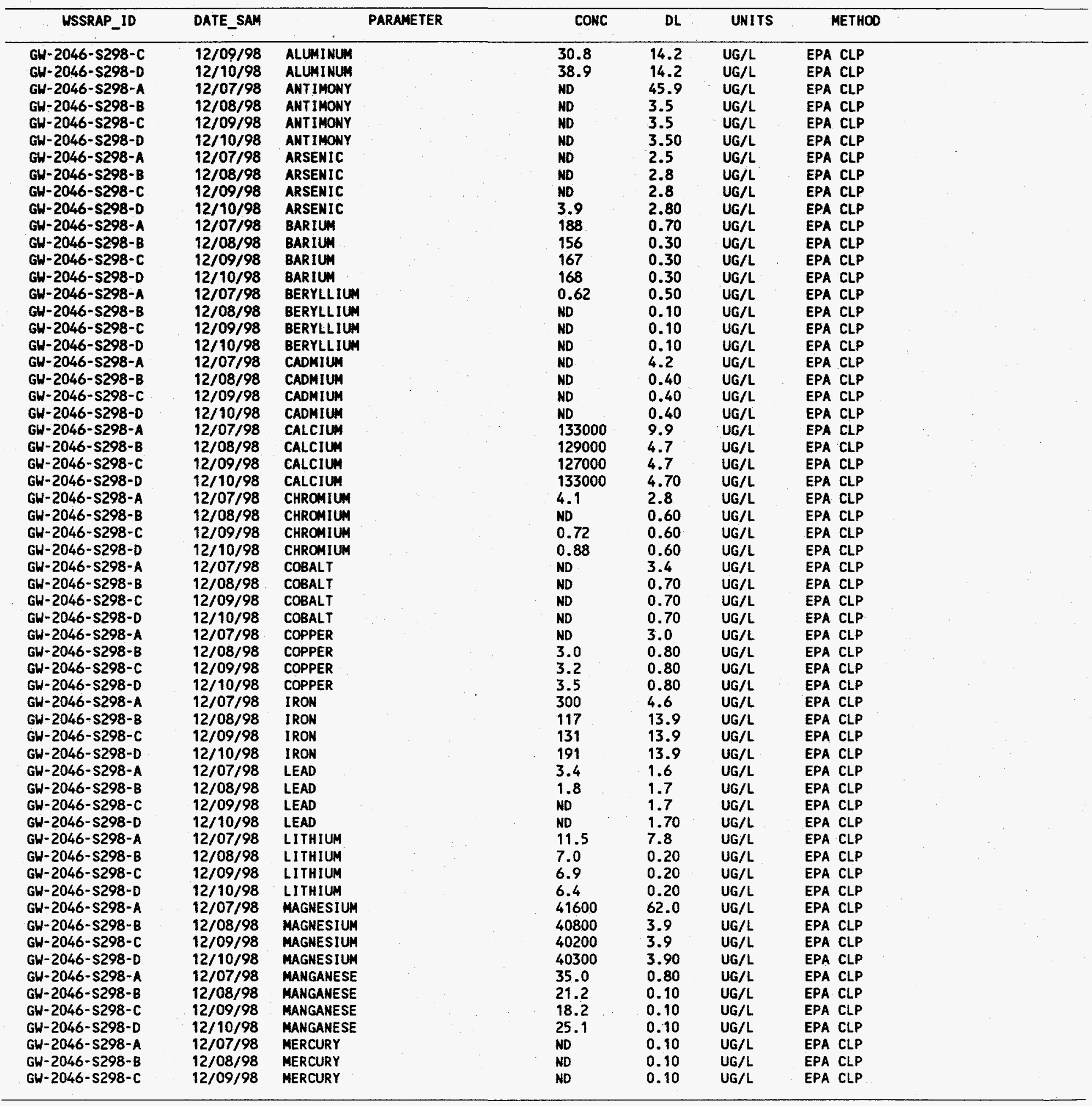




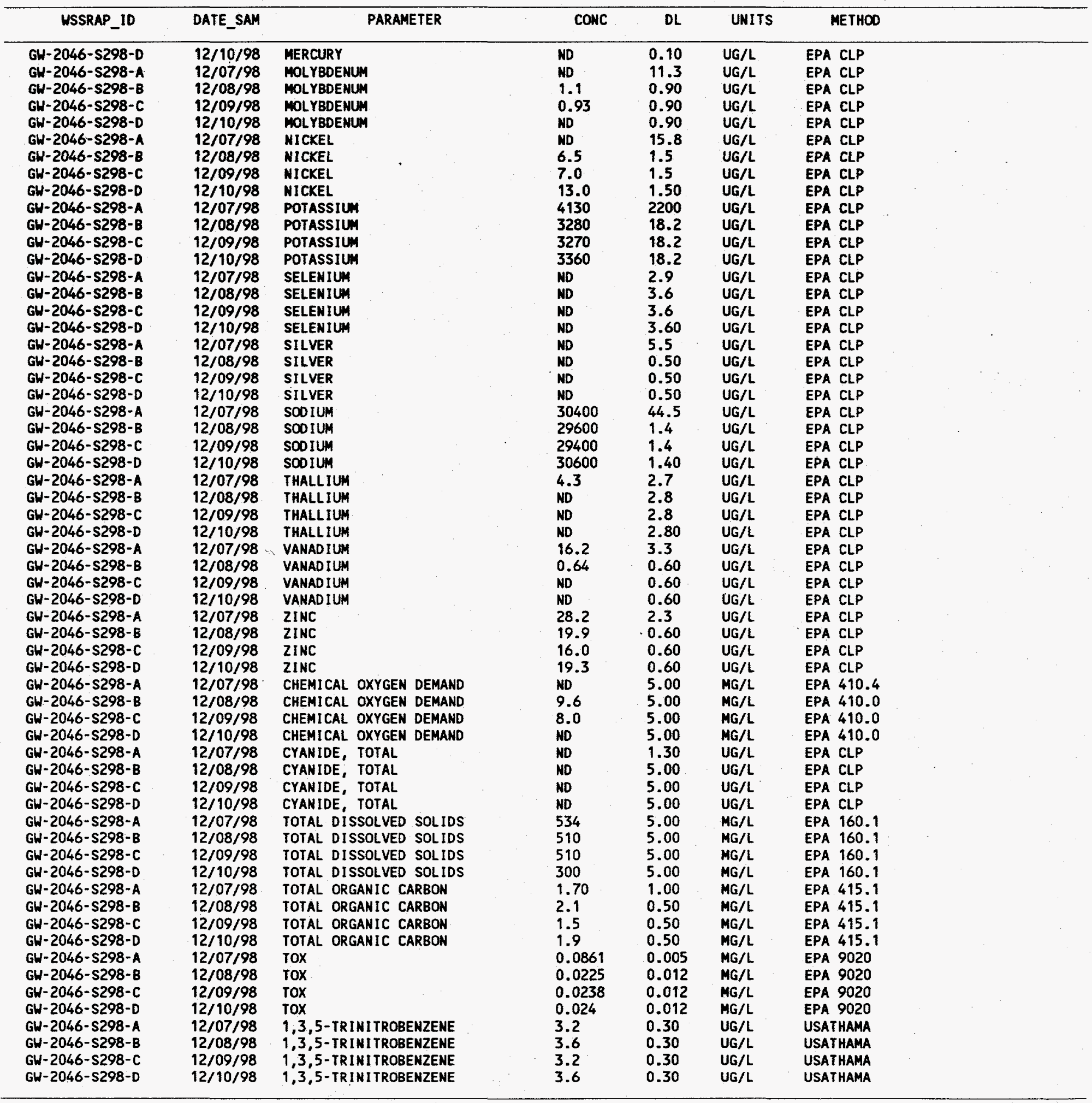




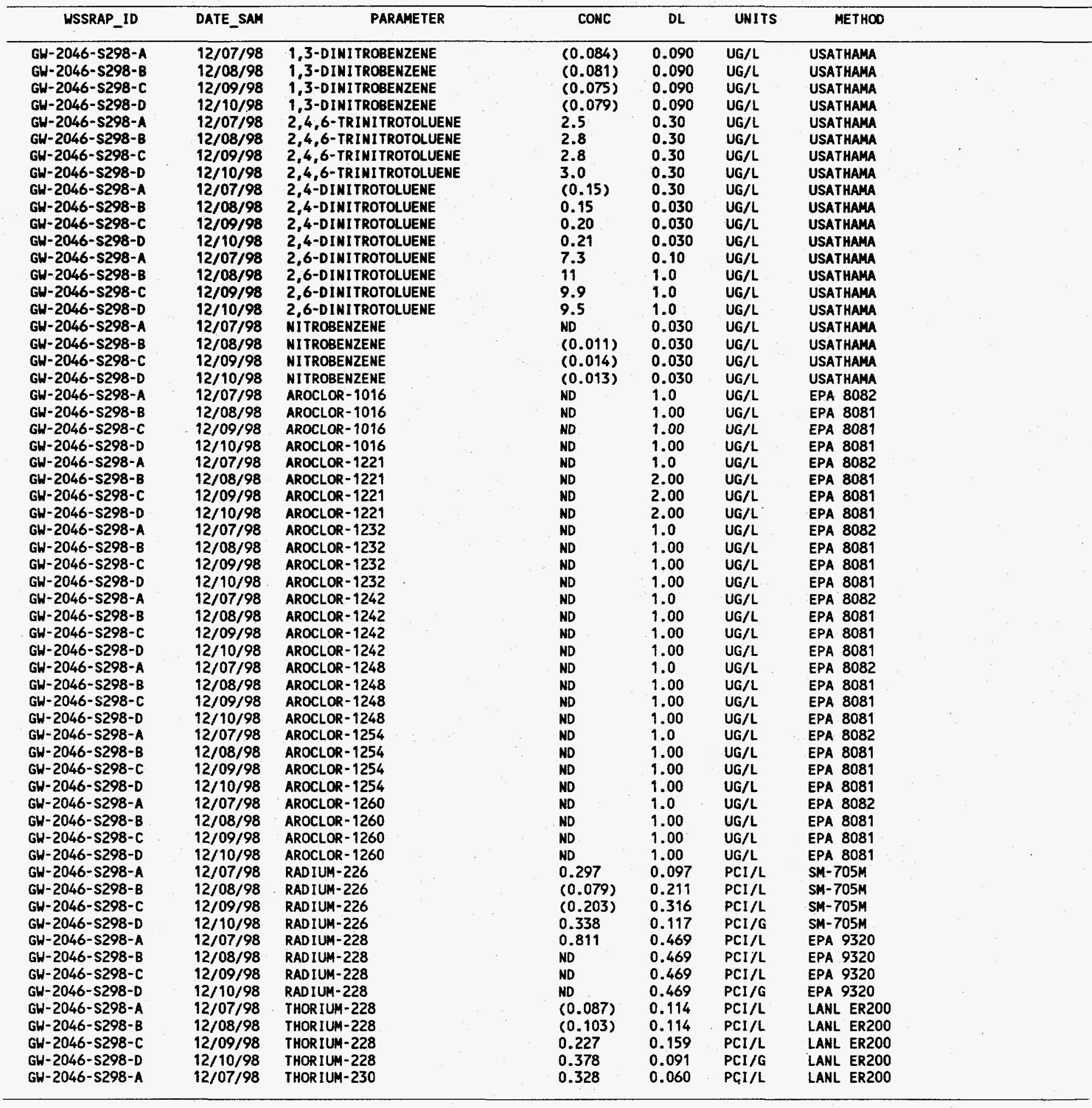




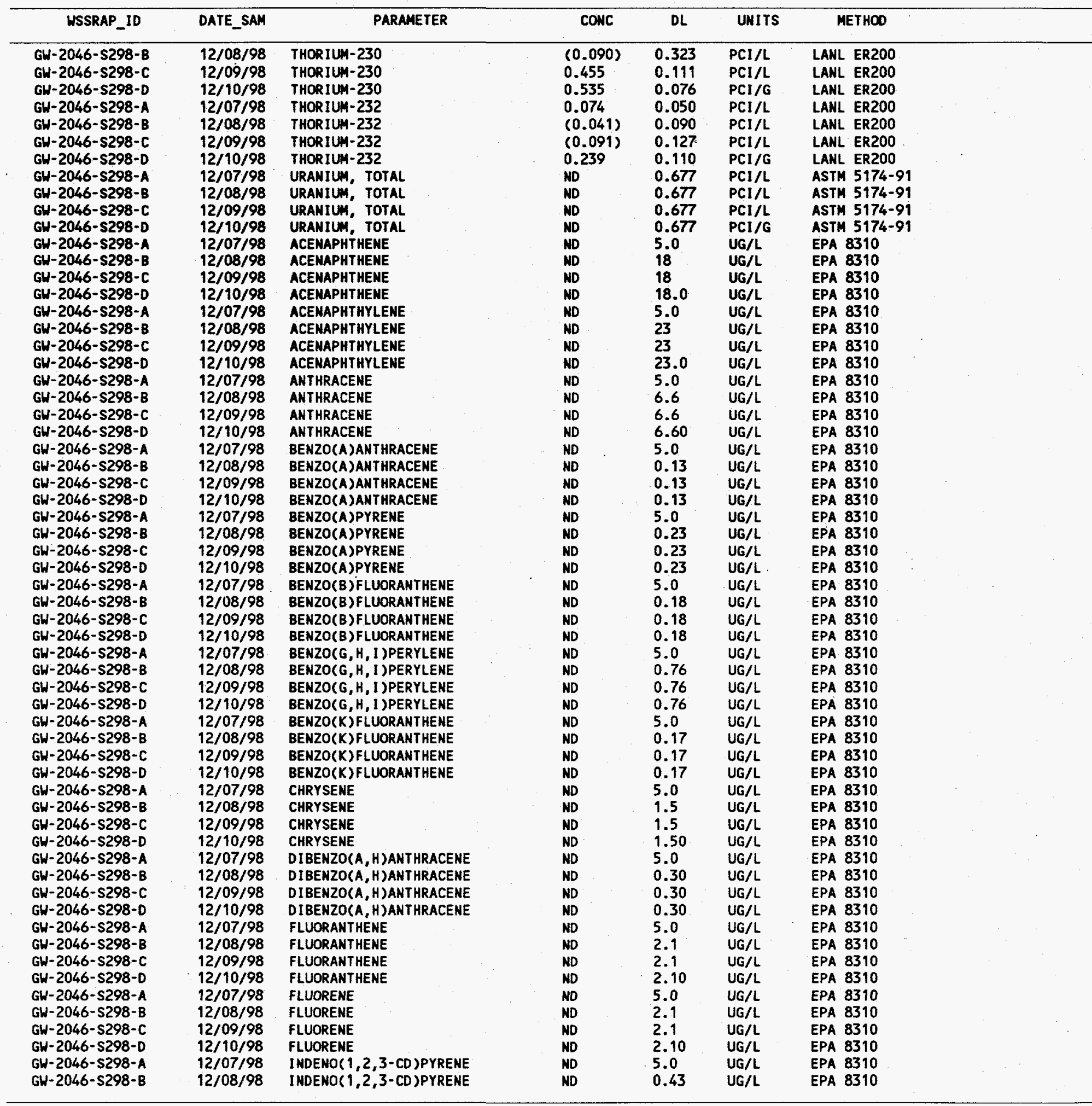




\begin{tabular}{|c|c|c|c|c|c|c|}
\hline WSSRAP_ID & DATE_SAM & PARAMETER & CONC & DL & UNITS & METHOD \\
\hline 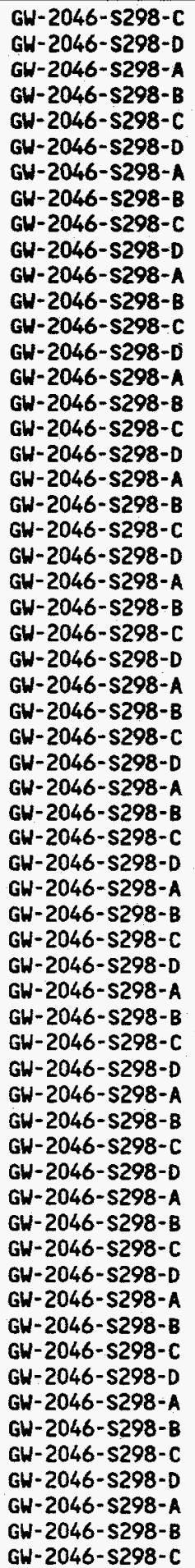 & $\begin{array}{l}12 / 09 / 98 \\
12 / 10 / 98 \\
12 / 07 / 98 \\
12 / 08 / 98 \\
12 / 09 / 98 \\
12 / 10 / 98 \\
12 / 07 / 98 \\
12 / 08 / 98 \\
12 / 09 / 98 \\
12 / 10 / 98 \\
12 / 07 / 98 \\
12 / 08 / 98 \\
12 / 09 / 98 \\
12 / 10 / 98 \\
12 / 07 / 98 \\
12 / 08 / 98 \\
12 / 09 / 98 \\
12 / 10 / 98 \\
12 / 07 / 98 \\
12 / 08 / 98 \\
12 / 09 / 98 \\
12 / 10 / 98 \\
12 / 07 / 98 \\
12 / 08 / 98 \\
12 / 09 / 98 \\
12 / 10 / 98 \\
12 / 07 / 98 \\
12 / 08 / 98 \\
12 / 09 / 98 \\
12 / 10 / 98 \\
12 / 07 / 98 \\
12 / 08 / 98 \\
12 / 09 / 98 \\
12 / 10 / 98 \\
12 / 07 / 98 \\
12 / 08 / 98 \\
12 / 09 / 98 \\
12 / 10 / 98 \\
12 / 07 / 98 \\
12 / 08 / 98 \\
12 / 09 / 98 \\
12 / 10 / 98 \\
12 / 07 / 98 \\
12 / 08 / 98 \\
12 / 09 / 98 \\
12 / 10 / 98 \\
12 / 07 / 98 \\
12 / 08 / 98 \\
12 / 09 / 98 \\
12 / 10 / 98 \\
12 / 07 / 98 \\
12 / 08 / 98 \\
12 / 09 / 98 \\
12 / 10 / 98 \\
12 / 07 / 98 \\
12 / 08 / 98 \\
12 / 09 / 98 \\
12 / 10 / 98 \\
12 / 07 / 98 \\
12 / 08 / 98 \\
12 / 09 / 98\end{array}$ & 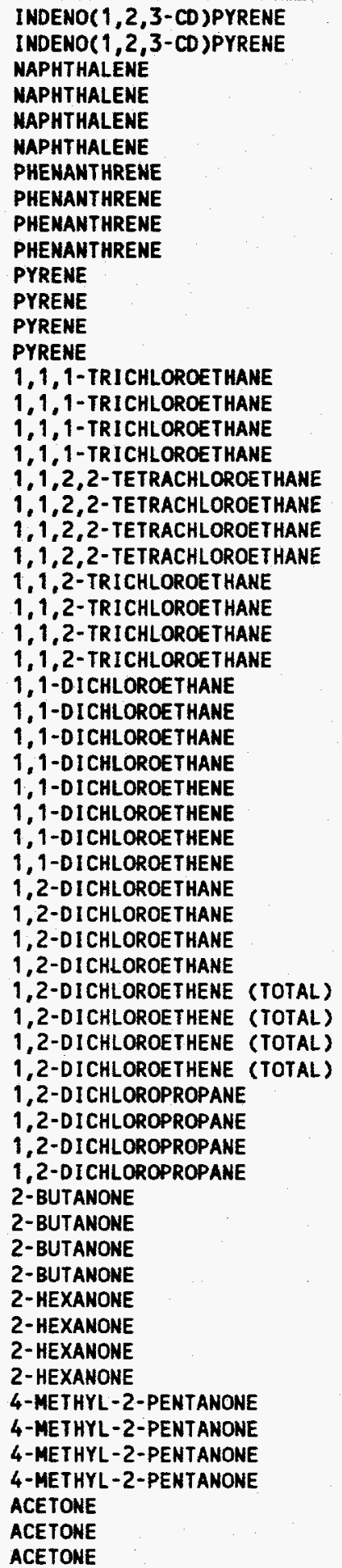 & 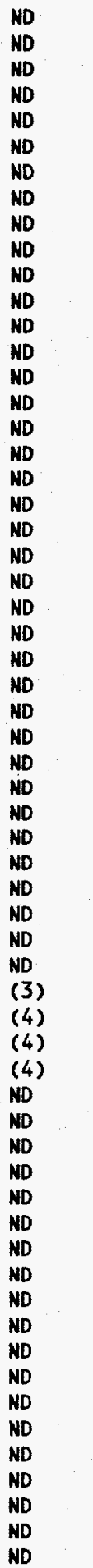 & $\begin{array}{l}0.43 \\
0.43 \\
5.0 \\
18 \\
18 \\
18.0 \\
5.0 \\
6.4 \\
6.4 \\
6.40 \\
5.0 \\
2.7 \\
2.7 \\
2.70 \\
10 \\
10 \\
10 \\
10 \\
10 \\
10 \\
10 \\
10 \\
10 \\
10 \\
10 \\
10 \\
10 \\
10 \\
10 \\
10 \\
10 \\
10 \\
10 \\
10 \\
10 \\
10 \\
10 \\
10 \\
10 \\
10 \\
10 \\
10 \\
10 \\
10 \\
10 \\
10 \\
10 \\
10 \\
10 \\
10 \\
10\end{array}$ & $\begin{array}{l}U G / L \\
U G / L \\
U G / L \\
U G / L \\
U G / L \\
U G / L \\
U G / L \\
U G / L \\
U G / L \\
U G / L \\
U G / L \\
U G / L \\
U G / L \\
U G / L \\
U G / L \\
U G / L \\
U G / L \\
U G / L \\
U G / L \\
U G / L \\
U G / L \\
U G / L \\
U G / L \\
U G / L \\
U G / L \\
U G / L \\
U G / L \\
U G / L \\
U G / L \\
U G / L \\
U G / L \\
U G / L \\
U G / L \\
U G / L \\
U G / L \\
U G / L \\
U G / L \\
U G / L \\
U G / L \\
U G / L \\
U G / L \\
U G / L \\
U G / L \\
U G / L \\
U G / L \\
U G / L \\
U G / L \\
U G / L \\
U G / L \\
U G / L \\
U G / L \\
U G / L \\
U G / L \\
U G / L \\
U G / L \\
U G / L \\
U G / L \\
U G / L \\
U G / L \\
U G / L \\
U G / L \\
\text { U. } \\
U\end{array}$ & $\begin{array}{l}\text { EPA } 8310 \\
\text { EPA } 8310 \\
\text { EPA } 8310 \\
\text { EPA } 8310 \\
\text { EPA } 8310 \\
\text { EPA } 8310 \\
\text { EPA } 8310 \\
\text { EPA } 8310 \\
\text { EPA } 8310 \\
\text { EPA } 8310 \\
\text { EPA } 8310 \\
\text { EPA } 8310 \\
\text { EPA } 8310 \\
\text { EPA } 8310 \\
\text { EPA CLP } \\
\text { EPA CLP } \\
\text { EPA CLP } \\
\text { EPA CLP } \\
\text { EPA CLP } \\
\text { EPA CLP } \\
\text { EPA CLP } \\
\text { EPA CLP } \\
\text { EPA CLP } \\
\text { EPA CLP } \\
\text { EPA CLP } \\
\text { EPA CLP } \\
\text { EPA CLP } \\
\text { EPA CLP } \\
\text { EPA CLP } \\
\text { EPA CLP } \\
\text { EPA CLP } \\
\text { EPA CLP } \\
\text { EPA CLP } \\
\text { EPA CLP } \\
\text { EPA CLP } \\
\text { EPA CLP } \\
\text { EPA CLP } \\
\text { EPA CLA CLP } \\
\text { EPA CLP } \\
\text { EPA CLP } \\
\text { EPA CLP } \\
\text { EPA CLP }\end{array}$ \\
\hline
\end{tabular}




\begin{tabular}{|c|c|c|c|c|c|c|}
\hline USSRAP_ID & DATE_SAM & PARAMETER & conc & DL & UNITS & METHOD \\
\hline 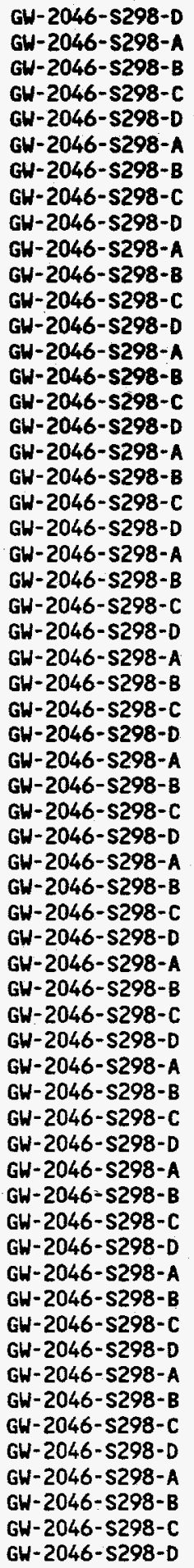 & $\begin{array}{l}12 / 10 / 98 \\
12 / 07 / 98 \\
12 / 08 / 98 \\
12 / 09 / 98 \\
12 / 10 / 98 \\
12 / 07 / 98 \\
12 / 08 / 98 \\
12 / 09 / 98 \\
12 / 10 / 98 \\
12 / 07 / 98 \\
12 / 08 / 98 \\
12 / 09 / 98 \\
12 / 10 / 98 \\
12 / 07 / 98 \\
12 / 08 / 98 \\
12 / 09 / 98 \\
12 / 10 / 98 \\
12 / 07 / 98 \\
12 / 08 / 98 \\
12 / 09 / 98 \\
12 / 10 / 98 \\
12 / 07 / 98 \\
12 / 08 / 98 \\
12 / 09 / 98 \\
12 / 10 / 98 \\
12 / 07 / 98 \\
12 / 08 / 98 \\
12 / 09 / 98 \\
12 / 10 / 98 \\
12 / 07 / 98 \\
12 / 08 / 98 \\
12 / 09 / 98 \\
12 / 10 / 98 \\
12 / 07 / 98 \\
12 / 08 / 98 \\
12 / 09 / 98 \\
12 / 10 / 98 \\
12 / 07 / 98 \\
12 / 08 / 98 \\
12 / 09 / 98 \\
12 / 10 / 98 \\
12 / 07 / 98 \\
12 / 08 / 98 \\
12 / 09 / 98 \\
12 / 10 / 98 \\
12 / 07 / 98 \\
12 / 08 / 98 \\
12 / 09 / 98 \\
12 / 10 / 98 \\
12 / 07 / 98 \\
12 / 08 / 98 \\
12 / 09 / 98 \\
12 / 10 / 98 \\
12 / 07 / 98 \\
12 / 08 / 98 \\
12 / 09 / 98 \\
12 / 10 / 98 \\
12 / 07 / 98 \\
12 / 08 / 98 \\
12 / 09 / 98 \\
12 / 10 / 98\end{array}$ & $\begin{array}{l}\text { ACETONE } \\
\text { BENZENE } \\
\text { BENZENE } \\
\text { BENZENE } \\
\text { BENZENE } \\
\text { BROMODICHLOROMETHANE } \\
\text { BROMOOICHLOROMETHANE } \\
\text { BROMOOICHLOROMETHANE } \\
\text { BROMOOICHLOROMETHANE } \\
\text { BROMOFORM } \\
\text { BROMOFORM } \\
\text { BROMOFORM } \\
\text { BROMOFORM } \\
\text { BROMOMETHANE } \\
\text { BROMOMETHANE } \\
\text { BROMOMETHANE } \\
\text { BROMOMETHANE } \\
\text { CARBON DISULFIDE } \\
\text { CARBON DISULFIDE } \\
\text { CARBON DISULFIDE } \\
\text { CARBON DISULFIDE } \\
\text { CARBON TETRACHLOR IDE } \\
\text { CARBON TETRACHLORIDE } \\
\text { CARBON TETRACHLORIDE } \\
\text { CARBON TETRACHLORIDE } \\
\text { CHLOROBENZENE } \\
\text { CHLOROBENZENE } \\
\text { CHLOROBENZENE } \\
\text { CHLOROBENZENE } \\
\text { CHLOROETHANE } \\
\text { CHLOROETHANE } \\
\text { CHLOROETHANE } \\
\text { CHLOROETHANE } \\
\text { CHLOROFORM } \\
\text { CHLOROFORM } \\
\text { CHLOROFORM } \\
\text { CHLOROFORM } \\
\text { CHLOROMETHANE } \\
\text { CHLOROMETHANE } \\
\text { CHLOROMETHANE } \\
\text { CHLOROMETHANE } \\
\text { CIS-1,3-DICHLOROPROPENE } \\
\text { CIS-1,3-DICHLOROPROPENE } \\
\text { CIS-1,3-DICHLOROPROPENE } \\
\text { CIS-1,3-DI CHLOROPROPENE } \\
\text { DIBROMOCHLOROMETHANE } \\
\text { DIBROMOCHLOROMETHANE } \\
\text { DIBROMOCHLOROMETHANE } \\
\text { DIBROMOCHLOROMETHANE } \\
\text { ETHYL BENZENE } \\
\text { ETHYL BENZENE } \\
\text { ETHYL BENZENE } \\
\text { ETHYL BENZENE } \\
\text { METHYLENE CHLORIDE } \\
\text { METHYLENE CHLORIDE } \\
\text { METHYLENE CHLORIDE } \\
\text { METHYLENE CHLORIDE } \\
\text { STYRENE } \\
\text { STYRENE } \\
\text { STYRENE } \\
\text { STYRENE }\end{array}$ & $\begin{array}{l}\text { (4) } \\
\text { ND } \\
\text { ND } \\
\text { ND } \\
\text { ND } \\
\text { ND } \\
\text { ND } \\
\text { ND } \\
\text { ND } \\
\text { ND } \\
\text { ND } \\
\text { ND } \\
\text { ND } \\
\text { ND } \\
\text { ND } \\
\text { ND } \\
\text { ND } \\
\text { ND } \\
\text { ND } \\
\text { ND } \\
\text { ND } \\
\text { ND } \\
\text { ND } \\
\text { ND } \\
\text { ND } \\
\text { ND } \\
\text { ND } \\
\text { ND } \\
\text { ND } \\
\text { ND } \\
\text { ND } \\
\text { ND } \\
\text { ND } \\
\text { ND } \\
\text { (3) } \\
\text { ND } \\
\text { ND } \\
\text { ND } \\
\text { ND } \\
\text { ND } \\
\text { ND } \\
\text { ND } \\
\text { ND } \\
\text { ND } \\
\text { ND } \\
\text { ND } \\
\text { ND } \\
\text { ND } \\
\text { ND } \\
\text { ND } \\
\text { C4 } \\
\text { N) }\end{array}$ & $\begin{array}{l}10 \\
10 \\
10 \\
10 \\
10 \\
10 \\
10 \\
10 \\
10 \\
10 \\
10 \\
10 \\
10 \\
10 \\
10 \\
10 \\
10 \\
10 \\
10 \\
10 \\
10 \\
10 \\
10 \\
10 \\
10 \\
10 \\
10 \\
10 \\
10 \\
10 \\
10 \\
10 \\
10 \\
10 \\
10 \\
10 \\
10 \\
10 \\
10 \\
10 \\
10 \\
10 \\
10 \\
10 \\
10 \\
10 \\
10 \\
10 \\
10 \\
10 \\
10 \\
10 \\
10 \\
10 \\
10 \\
10 \\
10 \\
10 \\
10 \\
10 \\
10\end{array}$ & 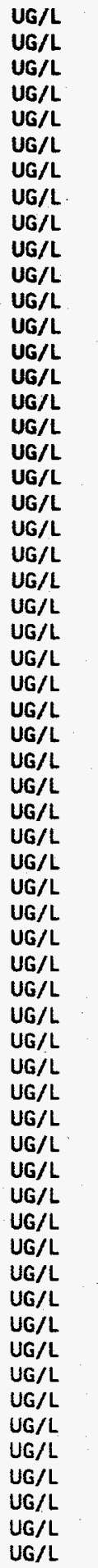 & 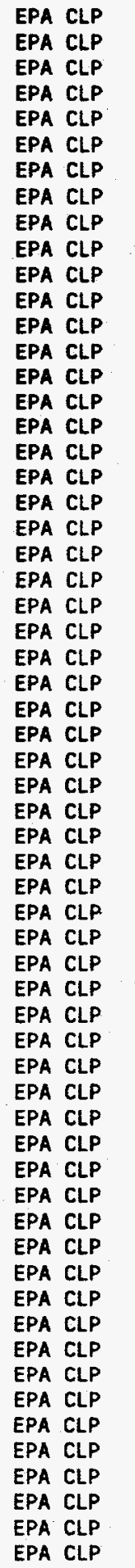 \\
\hline
\end{tabular}




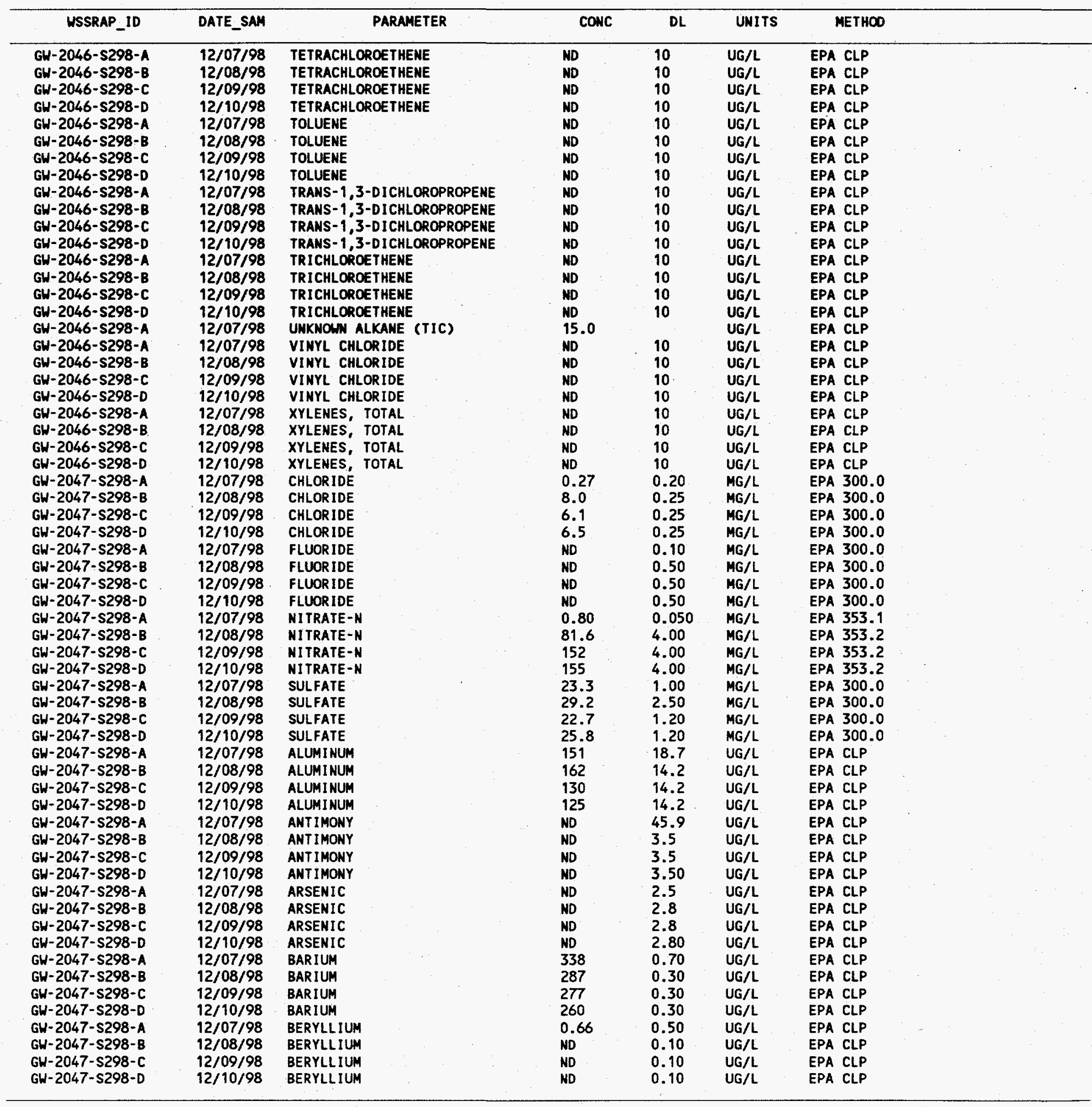




\begin{tabular}{|c|c|c|c|c|c|c|c|}
\hline WSSRAP_ID & DATE_SAM & PARAMETER & CONC & $D L$ & UNITS & METHOD & \\
\hline $\begin{array}{l}G W-2047-S 298-A \\
G W-2047-S 298-B \\
G W-2047-S 298-C \\
G W-2047-S 298-D \\
G W-2047-S 298-A \\
G W-2047-S 298-B \\
G W-2047-S 298-C \\
G W-2047-S 298-D \\
G W-2047-S 298-A \\
G W-2047-S 298-B \\
G W-2047-S 298-C \\
G W-2047-S 298-D \\
G W-2047-S 298-A \\
G W-2047-S 298-B \\
G W-2047-S 298-C \\
G W-2047-S 298-D \\
G W-2047-S 298-A \\
G W-2047-S 298-B \\
G W-2047-S 298-C \\
G W-2047-S 298-D \\
G W-2047-S 298-A \\
G W-2047-S 298-B \\
G W-2047-S 298-C \\
G W-2047-S 298-D \\
G W-2047-S 298-A \\
G W-2047-S 298-B \\
G W-2047-S 298-C \\
G W-2047-S 298-D \\
G H-2047-S 298-A \\
G W-2047-S 298-B \\
G W-2047-S 298-C \\
G W-2047-S 298-D \\
G W-2047-S 298-A \\
G W-2047-S 298-B \\
G W-2047-S 298-C \\
G W-2047-S 298-D \\
G W-2047-S 298-A \\
G W-2047-S 298-B \\
G W-2047-S 298-C \\
G W-2047-S 298-D \\
G W-2047-S 298-A \\
G W-2047-S 298-B \\
G W-2047-S 298-C \\
G W-2047-S 298-D \\
G W-2047-S 298-A \\
G W-2047-S 298-B \\
G W-2047-S 298-C \\
G W-2047-S 298-D \\
G H-2047-S 298-A \\
G W-2047-S 298-B \\
G W-2047-S 298-C \\
G W-2047-S 298-D \\
G W-2047-S 298-A \\
G W-2047-S 298-B \\
G W-2047-S 298-C \\
G W-2047-S 298-D \\
G W-2047-S 298-A \\
G W-2047-S 298-B \\
G W-2047-S 298-C \\
G W-2047-S 298-D \\
G W-2047-S 298-A\end{array}$ & $\begin{array}{l}12 / 07 / 98 \\
12 / 08 / 98 \\
12 / 09 / 98 \\
12 / 10 / 98 \\
12 / 07 / 98 \\
12 / 08 / 98 \\
12 / 09 / 98 \\
12 / 10 / 98 \\
12 / 07 / 98 \\
12 / 08 / 98 \\
12 / 09 / 98 \\
12 / 10 / 98 \\
12 / 07 / 98 \\
12 / 08 / 98 \\
12 / 09 / 98 \\
12 / 10 / 98 \\
12 / 07 / 98 \\
12 / 08 / 98 \\
12 / 09 / 98 \\
12 / 10 / 98 \\
12 / 07 / 98 \\
12 / 08 / 98 \\
12 / 09 / 98 \\
12 / 10 / 98 \\
12 / 07 / 98 \\
12 / 08 / 98 \\
12 / 09 / 98 \\
12 / 10 / 98 \\
12 / 07 / 98 \\
12 / 08 / 98 \\
12 / 09 / 98 \\
12 / 10 / 98 \\
12 / 07 / 98 \\
12 / 08 / 98 \\
12 / 09 / 98 \\
12 / 10 / 98 \\
12 / 07 / 98 \\
12 / 08 / 98 \\
12 / 09 / 98 \\
12 / 10 / 98 \\
12 / 07 / 98 \\
12 / 08 / 98 \\
12 / 09 / 98 \\
12 / 10 / 98 \\
12 / 07 / 98 \\
12 / 08 / 98 \\
12 / 09 / 98 \\
12 / 10 / 98 \\
12 / 07 / 98 \\
12 / 08 / 98 \\
12 / 09 / 98 \\
12 / 10 / 98 \\
12 / 07 / 98 \\
12 / 08 / 98 \\
12 / 09 / 98 \\
12 / 10 / 98 \\
12 / 07 / 98 \\
12 / 08 / 98 \\
12 / 09 / 98 \\
12 / 10 / 98 \\
12 / 07 / 98\end{array}$ & 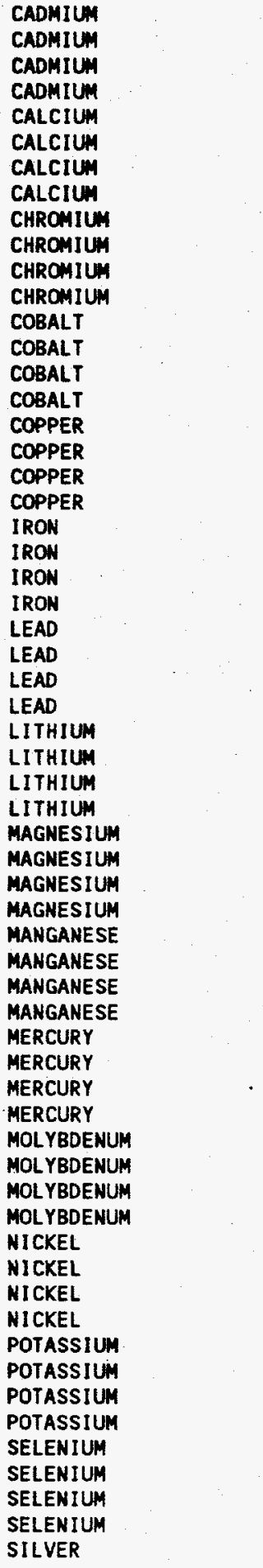 & 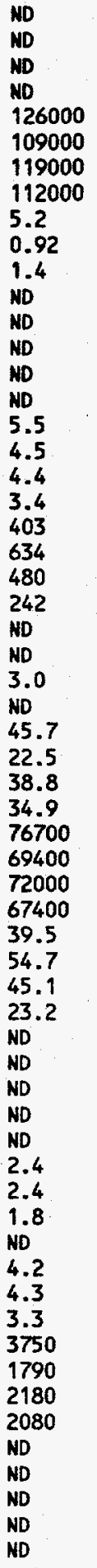 & $\begin{array}{l}4.2 \\
0.40 \\
0.40 \\
0.40 \\
9.9 \\
4.7 \\
4.7 \\
4.70 \\
2.8 \\
0.60 \\
0.60 \\
0.60 \\
3.4 \\
0.70 \\
0.70 \\
0.70 \\
3.0 \\
0.80 \\
0.80 \\
0.80 \\
4.6 \\
13.9 \\
13.9 \\
13.9 \\
1.6 \\
1.7 \\
1.7 \\
1.70 \\
7.8 \\
0.20 \\
0.20 \\
0.20 \\
62.0 \\
3.9 \\
3.9 \\
3.90 \\
0.80 \\
0.10 \\
0.10 \\
0.10 \\
0.10 \\
0.10 \\
0.10 \\
0.10 \\
11.3 \\
0.90 \\
0.90 \\
0.90 \\
15.8 \\
1.5 \\
1.5 \\
1.50 \\
2200 \\
18.2 \\
18.2 \\
18.2 \\
2.9 \\
3.6 \\
3.6 \\
3.60 \\
5.5 \\
\end{array}$ & 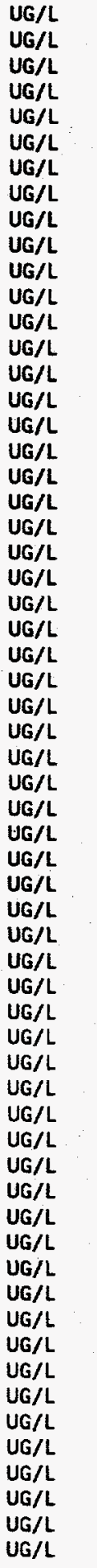 & 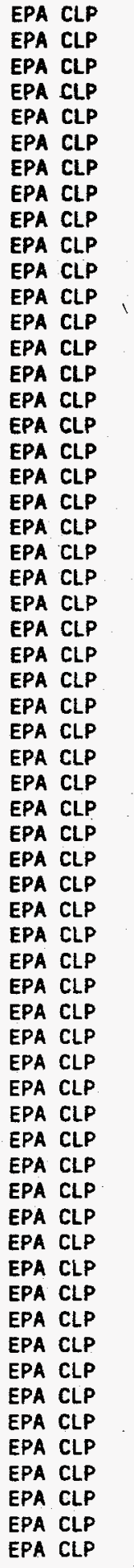 & . \\
\hline
\end{tabular}




\begin{tabular}{|c|c|c|c|c|c|c|}
\hline USSRAP_ID & DATE_SAM & PARAMETER & CONC & DL & \multicolumn{2}{|c|}{ UNITS METHOD } \\
\hline 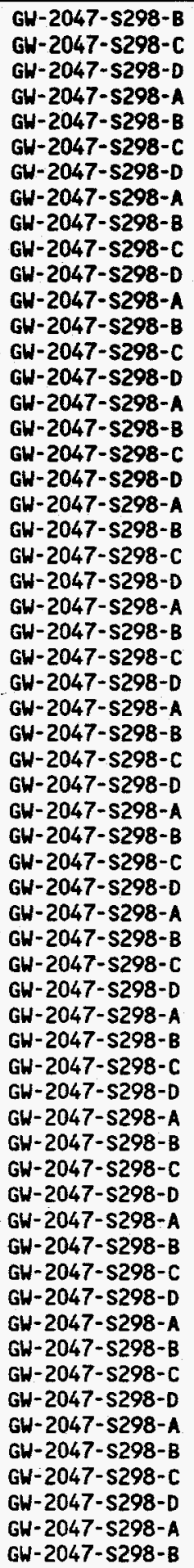 & $\begin{array}{l}12 / 08 / 98 \\
12 / 09 / 98 \\
12 / 10 / 98 \\
12 / 07 / 98 \\
12 / 08 / 98 \\
12 / 09 / 98 \\
12 / 10 / 98 \\
12 / 07 / 98 \\
12 / 08 / 98 \\
12 / 09 / 98 \\
12 / 10 / 98 \\
12 / 07 / 98 \\
12 / 08 / 98 \\
12 / 09 / 98 \\
12 / 10 / 98 \\
12 / 07 / 98 \\
12 / 08 / 98 \\
12 / 09 / 98 \\
12 / 10 / 98 \\
12 / 07 / 98 \\
12 / 08 / 98 \\
12 / 09 / 98 \\
12 / 10 / 98 \\
12 / 07 / 98 \\
12 / 08 / 98 \\
12 / 09 / 98 \\
12 / 10 / 98 \\
12 / 07 / 98 \\
12 / 08 / 98 \\
12 / 09 / 98 \\
12 / 10 / 98 \\
12 / 07 / 98 \\
12 / 08 / 98 \\
12 / 09 / 98 \\
12 / 10 / 98 \\
12 / 07 / 98 \\
12 / 08 / 98 \\
12 / 09 / 98 \\
12 / 10 / 98 \\
12 / 07 / 98 \\
12 / 08 / 98 \\
12 / 09 / 98 \\
12 / 10 / 98 \\
12 / 07 / 98 \\
12 / 08 / 98 \\
12 / 09 / 98 \\
12 / 10 / 98 \\
12 / 07 / 98 \\
12 / 08 / 98 \\
12 / 09 / 98 \\
12 / 10 / 98 \\
12 / 07 / 98 \\
12 / 08 / 98 \\
12 / 09 / 98 \\
12 / 10 / 98 \\
12 / 07 / 98 \\
12 / 08 / 98 \\
12 / 09 / 98 \\
12 / 10 / 98 \\
12 / 07 / 98 \\
12 / 08 / 98\end{array}$ & 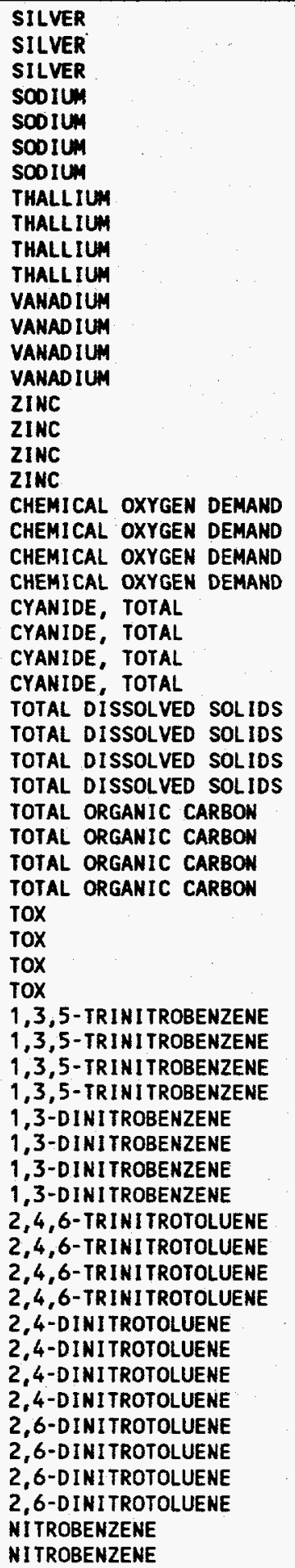 & 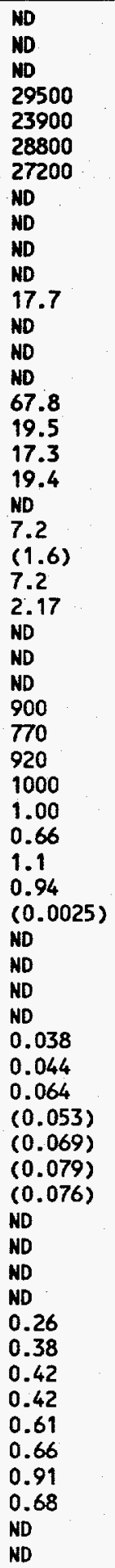 & $\begin{array}{l}0.50 \\
0.50 \\
0.50 \\
44.5 \\
1.4 \\
1.4 \\
1.40 \\
2.7 \\
2.8 \\
2.8 \\
2.80 \\
3.3 \\
0.60 \\
0.60 \\
0.60 \\
2.3 \\
0.60 \\
0.60 \\
0.60 \\
5.00 \\
5.00 \\
5.00 \\
5.00 \\
1.30 \\
5.0 \\
5.00 \\
5.00 \\
5.00 \\
5.00 \\
5.00 \\
5.00 \\
1.00 \\
0.50 \\
0.50 \\
0.50 \\
0.005 \\
0.012 \\
0.012 \\
0.012 \\
0.030 \\
0.030 \\
0.030 \\
0.030 \\
0.090 \\
0.090 \\
0.090 \\
0.090 \\
0.030 \\
0.030 \\
0.030 \\
0.030 \\
0.030 \\
0.030 \\
0.030 \\
0.030 \\
0.010 \\
0.010 \\
0.010 \\
0.010 \\
0.030 \\
0.030\end{array}$ & 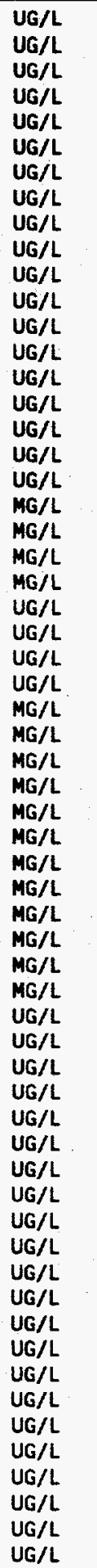 & $\begin{array}{l}\text { EPA CLP } \\
\text { EPA CLP } \\
\text { EPA CLP } \\
\text { EPA CLP } \\
\text { EPA CLP } \\
\text { EPA CLP } \\
\text { EPA CLP } \\
\text { EPA CLP } \\
\text { EPA CLP } \\
\text { EPA CLP } \\
\text { EPA CLP } \\
\text { EPA CLP } \\
\text { EPA CLP } \\
\text { EPA CLP } \\
\text { EPA CLP } \\
\text { EPA CLP } \\
\text { EPA CLP } \\
\text { EPA CLP } \\
\text { EPA CLP } \\
\text { EPA } 410.4 \\
\text { EPA } 410.0 \\
\text { EPA } 410.0 \\
\text { EPA } 410.0 \\
\text { EPA CLP } \\
\text { EPA CLP } \\
\text { EPA CLP } \\
\text { EPA CLP } \\
\text { EPA } 160.1 \\
\text { EPA } 160.1 \\
\text { EPA } 160.1 \\
\text { EPA } 160.1 \\
\text { EPA } 415.1 \\
\text { EPA } 415.1 \\
\text { EPA } 415.1 \\
\text { EPA } 415.1 \\
\text { EPA } 9020 \\
\text { EPA } 9020 \\
\text { EPA } 9020 \\
\text { EPA } 9020 \\
\text { USATHAMA } \\
\text { USATHAMA } \\
\text { USATHAMA } \\
\text { USATHAMA } \\
\text { USATHAMA } \\
\text { USATHAMA } \\
\text { USATHAMA } \\
\text { USATHAMA } \\
\text { USATHAMA } \\
\text { USATHAMA } \\
\text { USATHAMA } \\
\text { USATHAMA } \\
\text { USATHAMA } \\
\text { USATHAMA } \\
\text { USATHAMA } \\
\text { USATHAMA } \\
\text { USATHAMA } \\
\text { USATHAMA } \\
\text { USATHAMA } \\
\text { USATHAMA } \\
\text { USATHAMA } \\
\text { USATHAMA }\end{array}$ \\
\hline
\end{tabular}




\begin{tabular}{|c|c|c|c|c|c|c|}
\hline WSSRAP_ID & DATE_SAM & PARAMETER & CONC & $\mathrm{DL}$ & UNITS & METHOD \\
\hline 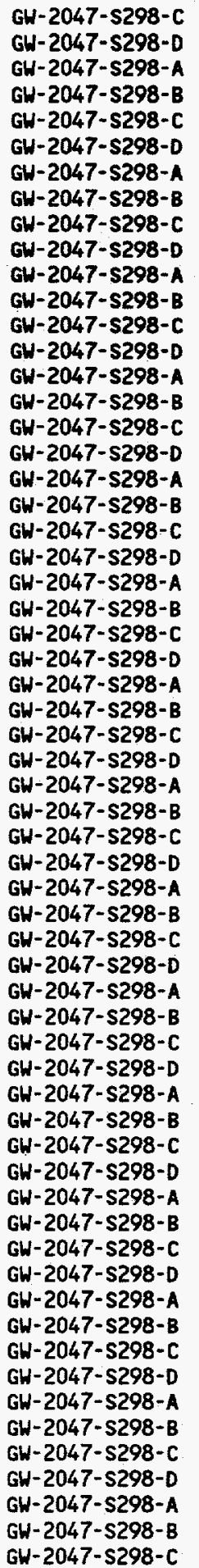 & $\begin{array}{l}12 / 09 / 98 \\
12 / 10 / 98 \\
12 / 07 / 98 \\
12 / 08 / 98 \\
12 / 09 / 98 \\
12 / 10 / 98 \\
12 / 07 / 98 \\
12 / 08 / 98 \\
12 / 09 / 98 \\
12 / 10 / 98 \\
12 / 07 / 98 \\
12 / 08 / 98 \\
12 / 09 / 98 \\
12 / 10 / 98 \\
12 / 07 / 98 \\
12 / 08 / 98 \\
12 / 09 / 98 \\
12 / 10 / 98 \\
12 / 07 / 98 \\
12 / 08 / 98 \\
12 / 09 / 98 \\
12 / 10 / 98 \\
12 / 07 / 98 \\
12 / 08 / 98 \\
12 / 09 / 98 \\
12 / 10 / 98 \\
12 / 07 / 98 \\
12 / 08 / 98 \\
12 / 09 / 98 \\
12 / 10 / 98 \\
12 / 07 / 98 \\
12 / 08 / 98 \\
12 / 09 / 98 \\
12 / 10 / 98 \\
12 / 07 / 98 \\
12 / 08 / 98 \\
12 / 09 / 98 \\
12 / 10 / 98 \\
12 / 07 / 98 \\
12 / 08 / 98 \\
12 / 09 / 98 \\
12 / 10 / 98 \\
12 / 07 / 98 \\
12 / 08 / 98 \\
12 / 09 / 98 \\
12 / 10 / 98 \\
12 / 07 / 98 \\
12 / 08 / 98 \\
12 / 09 / 98 \\
12 / 10 / 98 \\
12 / 07 / 98 \\
12 / 08 / 98 \\
12 / 09 / 98 \\
12 / 10 / 98 \\
12 / 07 / 98 \\
12 / 08 / 98 \\
12 / 09 / 98 \\
12 / 10 / 98 \\
12 / 07 / 98 \\
12 / 08 / 98 \\
12 / 09 / 98\end{array}$ & $\begin{array}{l}\text { NITROBENZENE } \\
\text { NI TROBENZENE } \\
\text { AROCLOR-1016 } \\
\text { AROCLOR-1016 } \\
\text { AROCLOR-1016 } \\
\text { AROCLOR-1016 } \\
\text { AROCLOR-1221 } \\
\text { AROCLOR-1221 } \\
\text { AROCLOR-1221 } \\
\text { AROCLOR-1221 } \\
\text { AROCLOR-1232 } \\
\text { AROCLOR-1232 } \\
\text { AROCLOR-1232 } \\
\text { AROCLOR-1232 } \\
\text { AROCLOR-1242 } \\
\text { AROCLOR-1242 } \\
\text { AROCLOR-1242 } \\
\text { AROCLOR-1242 } \\
\text { AROCLOR-1248 } \\
\text { AROCLOR-1248 } \\
\text { AROCLOR-1248 } \\
\text { AROCLOR-1248 } \\
\text { AROCLOR-1254 } \\
\text { AROCLOR-1254 } \\
\text { AROCLOR-1254 } \\
\text { AROCLOR-1254 } \\
\text { AROCLOR-1260 } \\
\text { AROCLOR-1260 } \\
\text { AROCLOR-1260 } \\
\text { AROCLOR-1260 } \\
\text { RADIUM-226 } \\
\text { RADIUM-226 } \\
\text { RADIUM-226 } \\
\text { RADIUM-226 } \\
\text { RADIUM-228 } \\
\text { RADIUM-228 } \\
\text { RADIUM-228 } \\
\text { RADIUM-228 } \\
\text { THORIUM-228 } \\
\text { THORIUM-228 } \\
\text { THORIUM-228 } \\
\text { THORIUM-228 } \\
\text { THORIUM-230 } \\
\text { THORIUM-230 } \\
\text { THORIUM- } 230 \\
\text { THORIUM-230 } \\
\text { THORIUM-232 } \\
\text { THORIUM-232 } \\
\text { THORIUM-232 } \\
\text { THORIUM-232 } \\
\text { URANIUM, TOTAL } \\
\text { URANIUM, TOTAL } \\
\text { URANIUM, TOTAL } \\
\text { URANIUM, TOTAL } \\
\text { ACENAPHTHENE } \\
\text { ACENAPHTHENE } \\
\text { ACENAPHTHENE } \\
\text { ACENAPHTHENE } \\
\text { ACENAPHTHYLENE } \\
\text { ACENAPHTHYLENE } \\
\text { ACENAPHTHYLENE }\end{array}$ & $\begin{array}{l}\text { ND } \\
\text { ND } \\
\text { ND } \\
\text { ND } \\
\text { ND } \\
\text { ND } \\
\text { ND } \\
\text { ND } \\
\text { ND } \\
\text { ND } \\
\text { ND } \\
\text { ND } \\
\text { ND } \\
\text { ND } \\
\text { ND } \\
\text { ND } \\
\text { ND } \\
\text { ND } \\
\text { ND } \\
\text { ND } \\
\text { ND } \\
\text { ND } \\
\text { ND } \\
\text { ND } \\
\text { ND } \\
\text { ND } \\
\text { ND } \\
\text { ND } \\
\text { ND } \\
\text { ND } \\
\text { C..342) } \\
0.534 \\
1.45 \\
0.839 \\
\text { ND } \\
\text { ND } \\
\text { ND } \\
\text { ND } \\
\text { C.031) } \\
0.268 \\
0.200 \\
0.421 \\
0.082 \\
0.291 \\
0.389 \\
(0.094) \\
\text { (0.015) } \\
\text { C.179) } \\
\text { C.032) } \\
\text { ND } \\
\text { ND } \\
\text { ND } \\
\text { ND } \\
\text { ND } \\
\text { ND } \\
\text { ND } \\
\text { ND } \\
\text { ND } \\
\text { ND } \\
\text { ND } \\
\text { ND } \\
\text { ND } \\
\text { ND } \\
\text { ND }\end{array}$ & 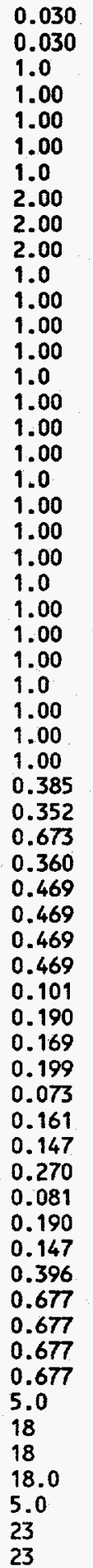 & $\begin{array}{l}U G / L \\
U G / L \\
U G / L \\
U G / L \\
U G / L \\
U G / L \\
U G / L \\
U G / L \\
U G / L \\
U G / L \\
U G / L \\
U G / L \\
U G / L \\
U G / L \\
U G / L \\
U G / L \\
U G / L \\
U G / L \\
U G / L \\
U G / L \\
U G / L \\
U G / L \\
U G / L \\
U G / L \\
U G / L \\
U G / L \\
U G / L \\
U G / L \\
U G / L \\
U G / L \\
P C I / L \\
P C I / L \\
P C I / L \\
P C I / G \\
P C I / L \\
P C I / L \\
P C I / L \\
P C I / G \\
P C I / L \\
P C I / L \\
P C I / L \\
P C I / G \\
P C I / L \\
P C I / L \\
P C I / L \\
P C I / G \\
P C I / L \\
P C I / L \\
P C I / L \\
P C I / G \\
P C I / L \\
P C I / L \\
P C I / L \\
P C I / G \\
U G / L \\
U G / L \\
U G / L \\
U G / L \\
U G / L \\
U G / L \\
U G / L\end{array}$ & $\begin{array}{l}\text { USATHAMA } \\
\text { USATHAMA } \\
\text { EPA } 8082 \\
\text { EPA } 8081 \\
\text { EPA } 8081 \\
\text { EPA } 8081 \\
\text { EPA } 8082 \\
\text { EPA } 8081 \\
\text { EPA } 8081 \\
\text { EPA } 8081 \\
\text { EPA } 8082 \\
\text { EPA } 8081 \\
\text { EPA } 8081 \\
\text { EPA } 8081 \\
\text { EPA } 8082 \\
\text { EPA } 8081 \\
\text { EPA } 8081 \\
\text { EPA } 8081 \\
\text { EPA } 8082 \\
\text { EPA } 8081 \\
\text { EPA } 8081 \\
\text { EPA } 8081 \\
\text { EPA } 8082 \\
\text { EPA } 8081 \\
\text { EPA } 8081 \\
\text { EPA } 8081 \\
\text { EPA } 8082 \\
\text { EPA } 8081 \\
\text { EPA } 8081 \\
\text { EPA } 8081 \\
\text { SM-705M } \\
\text { SM-705M } \\
\text { SM-705M } \\
\text { SM-705M } \\
\text { EPA } 9320 \\
\text { EPA } 9320 \\
\text { EPA } 9320 \\
\text { EPA } 9320 \\
\text { LANL ER200 } \\
\text { LANL ER200 } \\
\text { LANL ER200 } \\
\text { LANL ER200 } \\
\text { LANL ER200 } \\
\text { LANL ER200 } \\
\text { LANL ER200 } \\
\text { LANL ER200 } \\
\text { LANL ER200 } \\
\text { LANL ER200 } \\
\text { LANL ER200 } \\
\text { LANL ER200 } \\
\text { ASTM } 5174-91 \\
\text { ASTM } 5174-91 \\
\text { ASTM } 5174-91 \\
\text { ASTM } 5174-91 \\
\text { EPA } 8310 \\
\text { EPA } 8310 \\
\text { EPA } 8310 \\
\text { EPA } 8310 \\
\text { EPA } 8310 \\
\text { EPA } 8310 \\
\text { EPA } 8310 \\
\end{array}$ \\
\hline
\end{tabular}




\begin{tabular}{|c|c|c|c|c|c|c|}
\hline USSRAP_ID & DATE_SAM & PARAMETER & CONC & $\mathrm{DL}$ & UNITS & METHOO \\
\hline 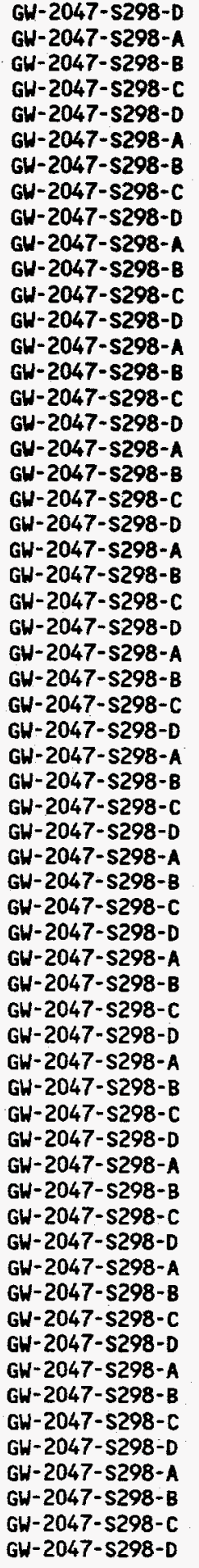 & $\begin{array}{l}12 / 10 / 98 \\
12 / 07 / 98 \\
12 / 08 / 98 \\
12 / 09 / 98 \\
12 / 10 / 98 \\
12 / 07 / 98 \\
12 / 08 / 98 \\
12 / 09 / 98 \\
12 / 10 / 98 \\
12 / 07 / 98 \\
12 / 08 / 98 \\
12 / 09 / 98 \\
12 / 10 / 98 \\
12 / 07 / 98 \\
12 / 08 / 98 \\
12 / 09 / 98 \\
12 / 10 / 98 \\
12 / 07 / 98 \\
12 / 08 / 98 \\
12 / 09 / 98 \\
12 / 10 / 98 \\
12 / 07 / 98 \\
12 / 08 / 98 \\
12 / 09 / 98 \\
12 / 10 / 98 \\
12 / 07 / 98 \\
12 / 08 / 98 \\
12 / 09 / 98 \\
12 / 10 / 98 \\
12 / 07 / 98 \\
12 / 08 / 98 \\
12 / 09 / 98 \\
12 / 10 / 98 \\
12 / 07 / 98 \\
12 / 08 / 98 \\
12 / 09 / 98 \\
12 / 10 / 98 \\
12 / 07 / 98 \\
12 / 08 / 98 \\
12 / 09 / 98 \\
12 / 10 / 98 \\
12 / 07 / 98 \\
12 / 08 / 98 \\
12 / 09 / 98 \\
12 / 10 / 98 \\
12 / 07 / 98 \\
12 / 08 / 98 \\
12 / 09 / 98 \\
12 / 10 / 98 \\
12 / 07 / 98 \\
12 / 08 / 98 \\
12 / 09 / 98 \\
12 / 10 / 98 \\
12 / 07 / 98 \\
12 / 08 / 98 \\
12 / 09 / 98 \\
12 / 10 / 98 \\
12 / 07 / 98 \\
12 / 08 / 98 \\
12 / 09 / 98 \\
12 / 10 / 98\end{array}$ & 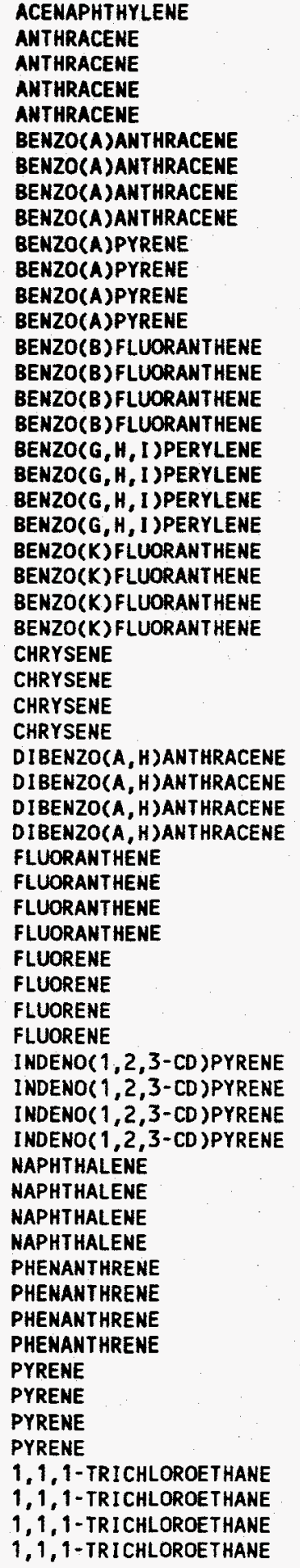 & $\begin{array}{l}\text { ND } \\
\text { ND } \\
\text { ND } \\
\text { ND } \\
\text { ND } \\
\text { ND } \\
\text { ND } \\
\text { ND } \\
\text { ND } \\
\text { ND } \\
\text { ND } \\
\text { ND } \\
\text { ND } \\
\text { ND } \\
\text { ND } \\
\text { ND } \\
\text { ND } \\
\text { ND } \\
\text { ND } \\
\text { ND } \\
\text { ND } \\
\text { ND } \\
\text { ND } \\
\text { ND } \\
\text { ND } \\
\text { ND } \\
\text { ND } \\
\text { ND } \\
\text { ND } \\
\text { ND } \\
\text { ND } \\
\text { ND } \\
\text { ND } \\
\text { ND } \\
\text { ND } \\
\text { ND } \\
\text { ND } \\
\text { ND } \\
\text { ND } \\
\text { ND } \\
\text { ND } \\
\text { ND } \\
\text { ND } \\
\text { ND } \\
\text { ND } \\
\text { ND } \\
\text { ND } \\
\text { ND } \\
\text { ND } \\
\text { ND } \\
\text { ND } \\
\text { ND } \\
\text { ND } \\
\text { ND } \\
\text { ND } \\
\text { ND } \\
\text { ND }\end{array}$ & $\begin{array}{l}23.0 \\
5.0 \\
6.6 \\
6.6 \\
6.60 \\
5.0 \\
0.13 \\
0.13 \\
0.13 \\
5.0 \\
0.23 \\
0.23 \\
0.23 \\
5.0 \\
0.18 \\
0.18 \\
0.18 \\
5.0 \\
0.76 \\
0.76 \\
0.76 \\
5.0 \\
0.17 \\
0.17 \\
0.17 \\
5.0 \\
1.5 \\
1.5 \\
1.50 \\
5.0 \\
0.30 \\
0.30 \\
0.30 \\
5.0 \\
2.1 \\
2.1 \\
2.10 \\
5.0 \\
2.1 \\
2.1 \\
2.10 \\
5.0 \\
0.43 \\
0.43 \\
0.43 \\
5.0 \\
18 \\
18 \\
18.0 \\
5.0 \\
6.4 \\
6.4 \\
6.40 \\
5.0 \\
2.7 \\
2.7 \\
2.70 \\
10 \\
10 \\
10 \\
10 \\
\end{array}$ & $\begin{array}{l}\text { UG/L } \\
U G / L \\
U G / L \\
U G / L \\
U G / L \\
U G / L \\
U G / L \\
U G / L \\
U G / L \\
U G / L \\
U G / L \\
U G / L \\
U G / L \\
U G / L \\
U G / L \\
U G / L \\
U G / L \\
U G / L \\
U G / L \\
U G / L \\
U G / L \\
U G / L \\
U G / L \\
U G / L \\
U G / L \\
U G / L \\
U G / L \\
U G / L \\
U G / L \\
U G / L \\
U G / L \\
U G / L \\
U G / L \\
U G / L \\
U G / L \\
U G / L \\
U G / L \\
U G / L \\
U G / L \\
U G / L \\
U G / L \\
U G / L \\
U G / L \\
U G / L \\
U G / L \\
U G / L \\
U G / L \\
U G / L \\
U G / L \\
U G / L \\
U G / L \\
U G / L \\
U G / L \\
U G / L \\
U G / L \\
U G / L \\
U G / L \\
U G / L \\
U G / L \\
U G / L \\
U G / L \\
\text { US }\end{array}$ & 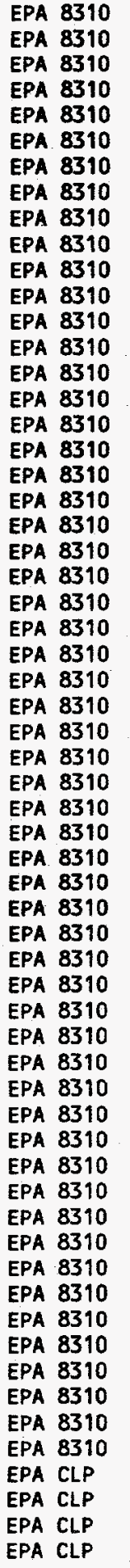 \\
\hline
\end{tabular}




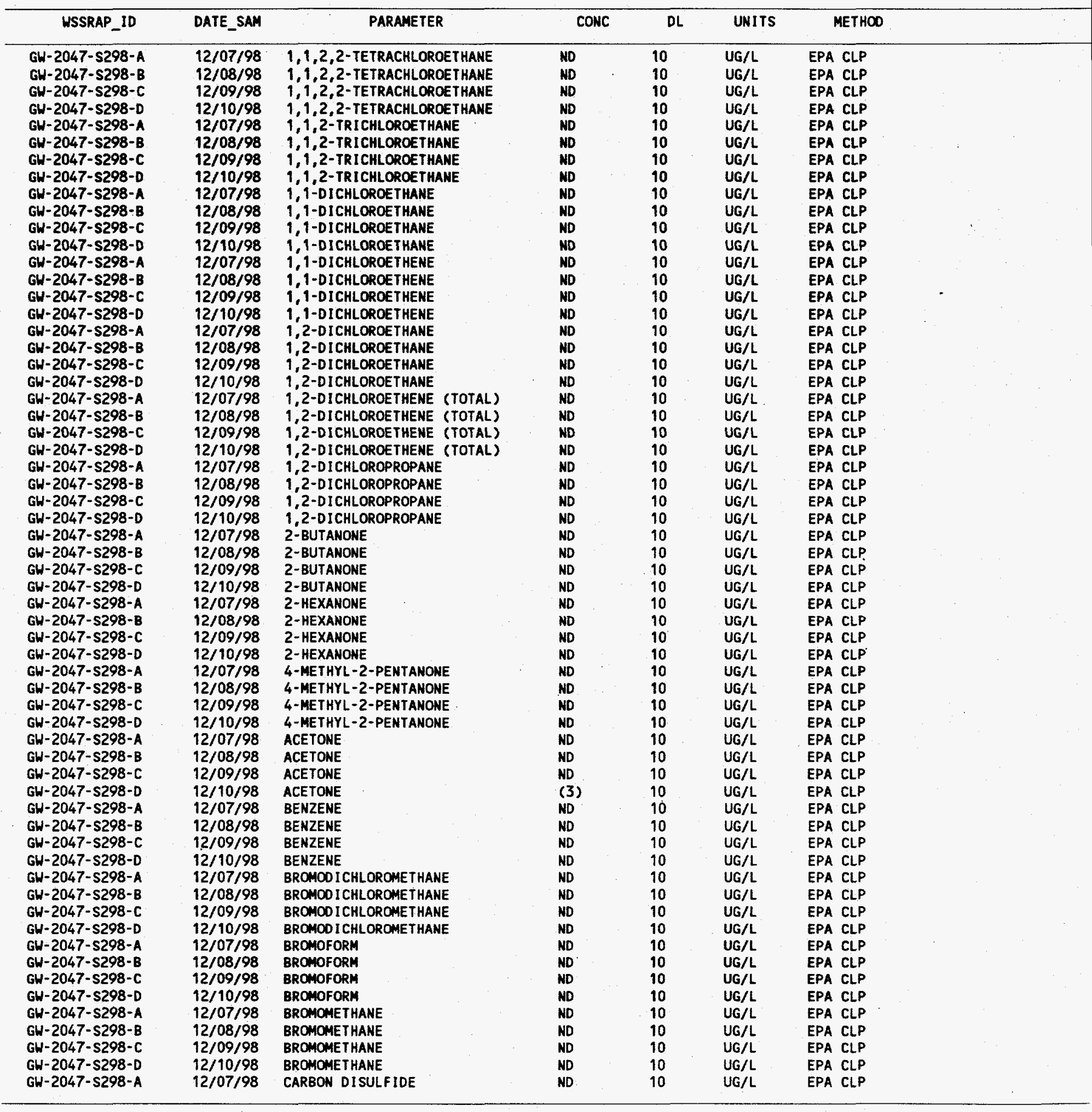




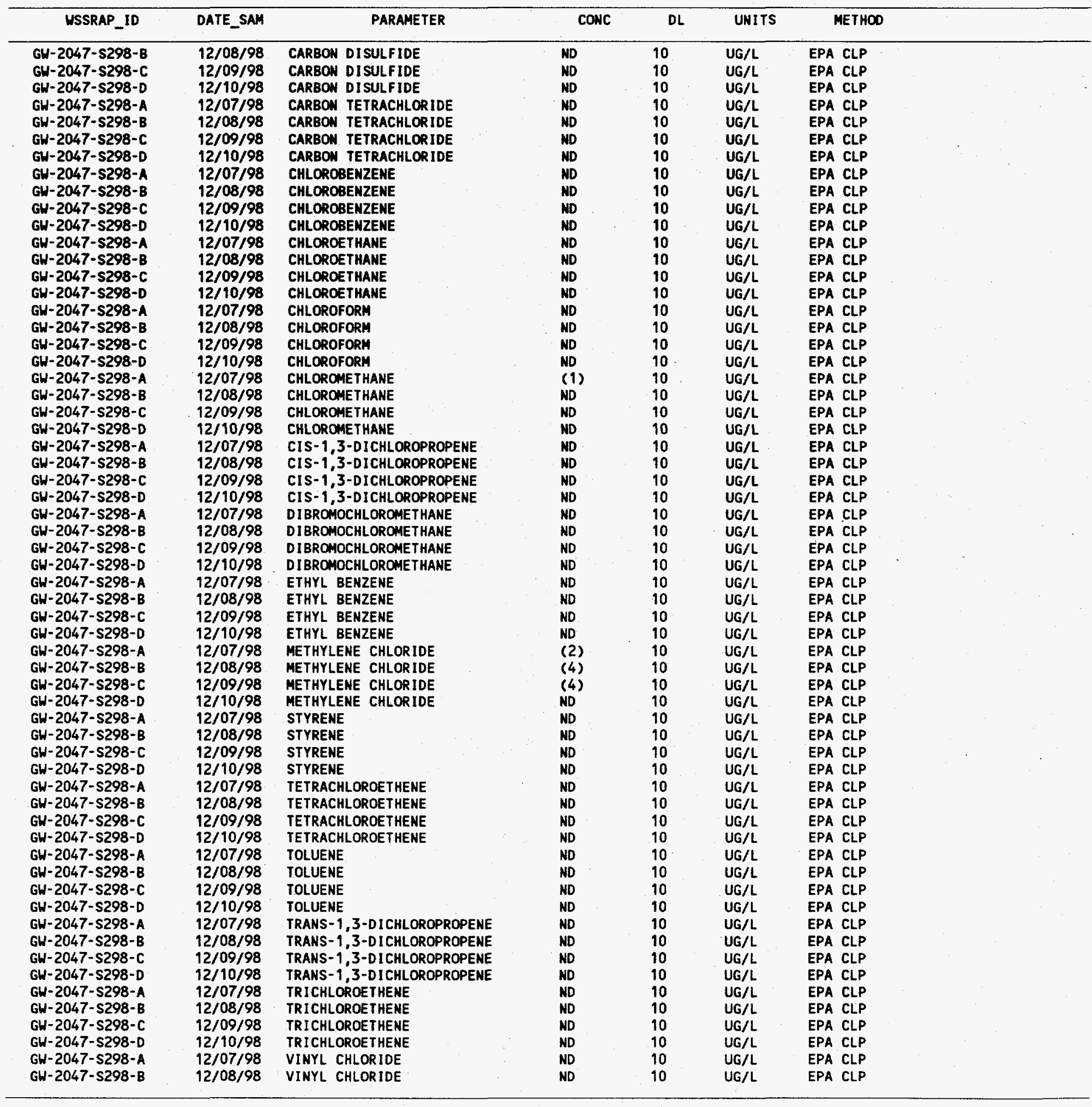




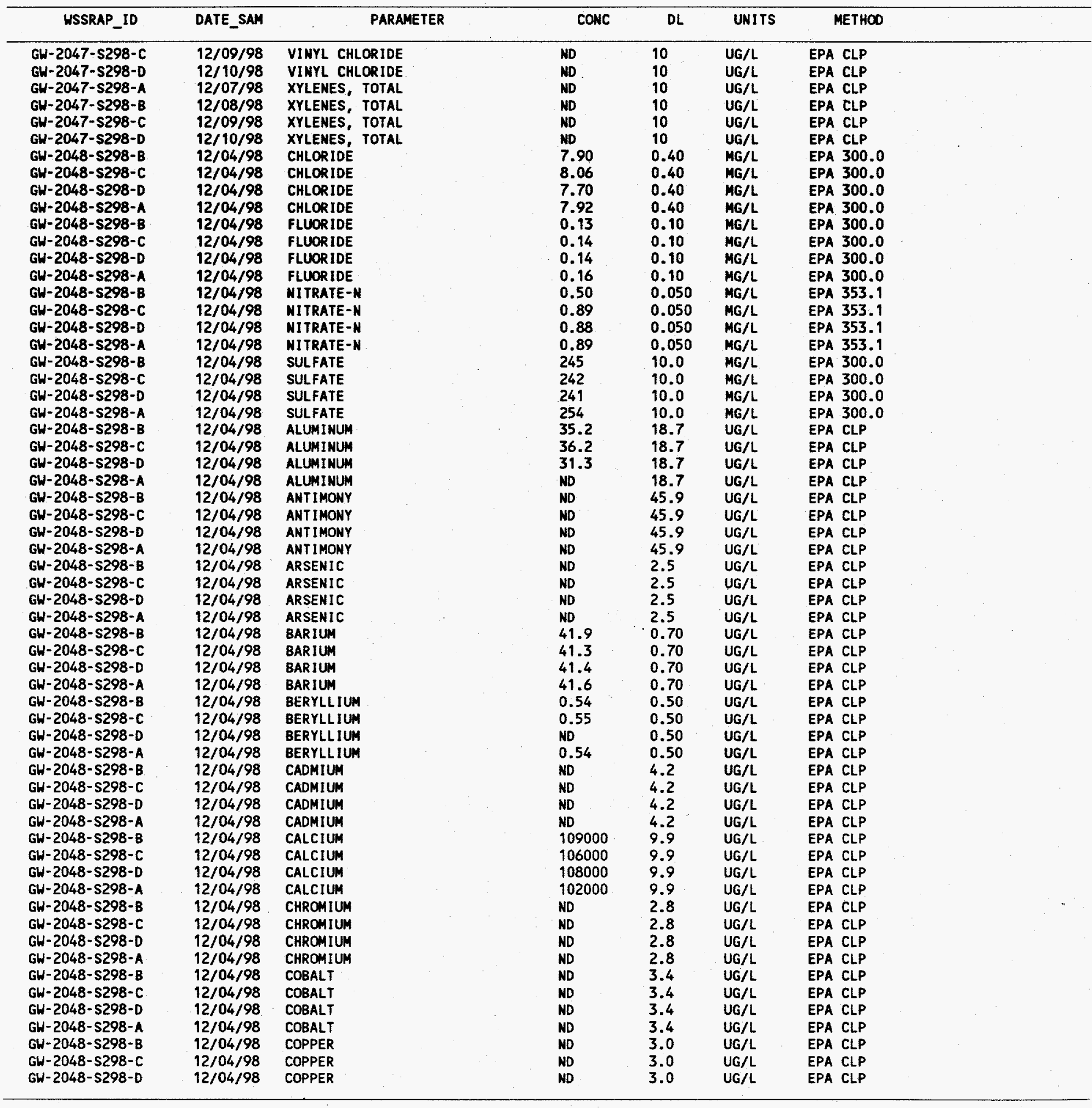




\begin{tabular}{|c|c|c|c|c|c|c|}
\hline USSRAP_ID & DATE_SAM & PARAMETER & CONC & $D L$ & UNITS & METHOD \\
\hline 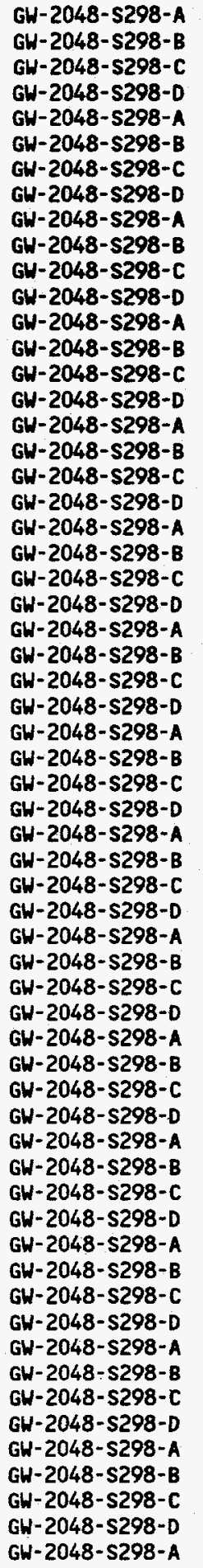 & $\begin{array}{l}12 / 04 / 98 \\
12 / 04 / 98 \\
12 / 04 / 98 \\
12 / 04 / 98 \\
12 / 04 / 98 \\
12 / 04 / 98 \\
12 / 04 / 98 \\
12 / 04 / 98 \\
12 / 04 / 98 \\
12 / 04 / 98 \\
12 / 04 / 98 \\
12 / 04 / 98 \\
12 / 04 / 98 \\
12 / 04 / 98 \\
12 / 04 / 98 \\
12 / 04 / 98 \\
12 / 04 / 98 \\
12 / 04 / 98 \\
12 / 04 / 98 \\
12 / 04 / 98 \\
12 / 04 / 98 \\
12 / 04 / 98 \\
12 / 04 / 98 \\
12 / 04 / 98 \\
12 / 04 / 98 \\
12 / 04 / 98 \\
12 / 04 / 98 \\
12 / 04 / 98 \\
12 / 04 / 98 \\
12 / 04 / 98 \\
12 / 04 / 98 \\
12 / 04 / 98 \\
12 / 04 / 98 \\
12 / 04 / 98 \\
12 / 04 / 98 \\
12 / 04 / 98 \\
12 / 04 / 98 \\
12 / 04 / 98 \\
12 / 04 / 98 \\
12 / 04 / 98 \\
12 / 04 / 98 \\
12 / 04 / 98 \\
12 / 04 / 98 \\
12 / 04 / 98 \\
12 / 04 / 98 \\
12 / 04 / 98 \\
12 / 04 / 98 \\
12 / 04 / 98 \\
12 / 04 / 98 \\
12 / 04 / 98 \\
12 / 04 / 98 \\
12 / 04 / 98 \\
12 / 04 / 98 \\
12 / 04 / 98 \\
12 / 04 / 98 \\
12 / 04 / 98 \\
12 / 04 / 98 \\
12 / 04 / 98 \\
12 / 04 / 98 \\
12 / 04 / 98 \\
12 / 04 / 98\end{array}$ & $\begin{array}{l}\text { COPPER } \\
\text { IRON } \\
\text { IRON } \\
\text { IRON } \\
\text { IRON } \\
\text { LEAD } \\
\text { LEAD } \\
\text { LEAD } \\
\text { LEAD } \\
\text { LITHIUM } \\
\text { LIITIUM } \\
\text { LITHIUM } \\
\text { LITHIUM } \\
\text { MAGNESIUM } \\
\text { MMGNESIUM } \\
\text { MAGNESIUM } \\
\text { MAGNESIUM } \\
\text { MANGANESE } \\
\text { MANGANESE } \\
\text { MANGANESE } \\
\text { MANGANESE } \\
\text { MERCURY } \\
\text { MERCURY } \\
\text { MERCURY } \\
\text { MERCURY } \\
\text { MOLYBDENUM } \\
\text { MOLYBDENUM } \\
\text { MOLYBDENUM } \\
\text { MOLYBDENUM } \\
\text { NICKEL } \\
\text { NICKEL } \\
\text { NICKEL } \\
\text { NICKEL } \\
\text { POTASSIUM } \\
\text { POTASSIUM } \\
\text { POTASSIUM } \\
\text { POTASSIUM } \\
\text { SELENIUM } \\
\text { SELENIUM } \\
\text { SELENIUM } \\
\text { SELENIUM } \\
\text { SILVER } \\
\text { SILVER } \\
\text { SILVER } \\
\text { SILVER } \\
\text { SODIUM } \\
\text { SODIUM } \\
\text { SODIUM } \\
\text { SOOIUM } \\
\text { THALLIUM } \\
\text { THALLIUM } \\
\text { THALLIUM } \\
\text { THALLIUM } \\
\text { VANADIUM } \\
\text { VANADIUM } \\
\text { VANADIUM } \\
\text { VANADIUM } \\
\text { ZINC } \\
\text { ZINC } \\
\text { ZINC } \\
\text { ZINC }\end{array}$ & $\begin{array}{l}\text { ND } \\
45.6 \\
38.2 \\
41.4 \\
76.9 \\
\text { ND } \\
\text { ND } \\
2.6 \\
\text { ND } \\
14.2 \\
17.1 \\
15.6 \\
18.0 \\
43500 \\
41800 \\
42600 \\
40400 \\
4.8 \\
4.6 \\
4.7 \\
6.0 \\
\text { ND } \\
\text { ND } \\
\text { ND } \\
\text { ND } \\
\text { ND } \\
\text { ND } \\
\text { ND } \\
\text { ND } \\
\text { ND } \\
\text { ND } \\
\text { ND } \\
\text { ND } \\
3060 \\
4680 \\
2480 \\
\text { ND } \\
12.1 \\
12.2 \\
10.3 \\
11.8 \\
\text { ND } \\
\text { ND } \\
\text { ND } \\
\text { ND } \\
63200 \\
60500 \\
62000 \\
57500 \\
\text { ND } \\
4.7 \\
4.7 \\
5.8 \\
15.4 \\
15.9 \\
15.4 \\
15.8 \\
11.9 \\
11.2 \\
11.3 \\
11.2\end{array}$ & $\begin{array}{l}3.0 \\
4.6 \\
4.6 \\
4.6 \\
4.6 \\
1.6 \\
1.6 \\
1.6 \\
1.6 \\
7.8 \\
7.8 \\
7.8 \\
7.8 \\
62.0 \\
62.0 \\
62.0 \\
62.0 \\
0.80 \\
0.80 \\
0.80 \\
0.80 \\
0.10 \\
0.10 \\
0.10 \\
0.10 \\
11.3 \\
11.3 \\
11.3 \\
11.3 \\
15.8 \\
15.8 \\
15.8 \\
15.8 \\
2200 \\
2200 \\
2200 \\
2200 \\
2.9 \\
2.9 \\
2.9 \\
2.9 \\
5.5 \\
5.5 \\
5.5 \\
5.5 \\
44.5 \\
44.5 \\
44.5 \\
44.5 \\
2.7 \\
2.7 \\
2.7 \\
2.7 \\
3.3 \\
3.3 \\
3.3 \\
3.3 \\
2.3 \\
2.3 \\
2.3 \\
2.3\end{array}$ & 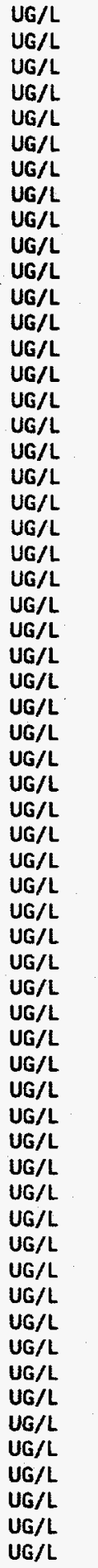 & 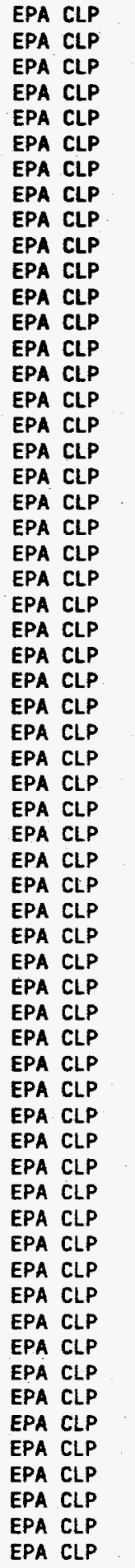 \\
\hline
\end{tabular}




\begin{tabular}{|c|c|c|c|c|c|c|}
\hline USSRAP_ID & DATE_SAM & PARAMETER & CONC & DL & UNITS & METHOD \\
\hline 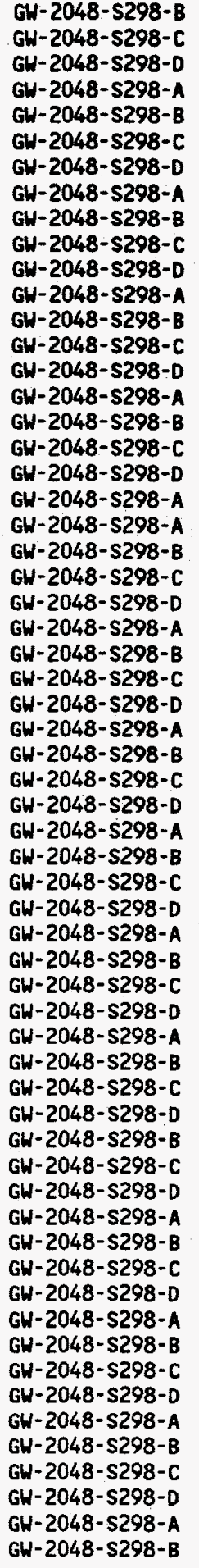 & $\begin{array}{l}12 / 04 / 98 \\
12 / 04 / 98 \\
12 / 04 / 98 \\
12 / 04 / 98 \\
12 / 04 / 98 \\
12 / 04 / 98 \\
12 / 04 / 98 \\
12 / 04 / 98 \\
12 / 04 / 98 \\
12 / 04 / 98 \\
12 / 04 / 98 \\
12 / 04 / 98 \\
12 / 04 / 98 \\
12 / 04 / 98 \\
12 / 04 / 98 \\
12 / 04 / 98 \\
12 / 04 / 98 \\
12 / 04 / 98 \\
12 / 04 / 98 \\
12 / 04 / 98 \\
12 / 04 / 98 \\
12 / 04 / 98 \\
12 / 04 / 98 \\
12 / 04 / 98 \\
12 / 04 / 98 \\
12 / 04 / 98 \\
12 / 04 / 98 \\
12 / 04 / 98 \\
12 / 04 / 98 \\
12 / 04 / 98 \\
12 / 04 / 98 \\
12 / 04 / 98 \\
12 / 04 / 98 \\
12 / 04 / 98 \\
12 / 04 / 98 \\
12 / 04 / 98 \\
12 / 04 / 98 \\
12 / 04 / 98 \\
12 / 04 / 98 \\
12 / 04 / 98 \\
12 / 04 / 98 \\
12 / 04 / 98 \\
12 / 04 / 98 \\
12 / 04 / 98 \\
12 / 04 / 98 \\
12 / 04 / 98 \\
12 / 04 / 98 \\
12 / 04 / 98 \\
12 / 04 / 98 \\
12 / 04 / 98 \\
12 / 04 / 98 \\
12 / 04 / 98 \\
12 / 04 / 98 \\
12 / 04 / 98 \\
12 / 04 / 98 \\
12 / 04 / 98 \\
12 / 04 / 98 \\
12 / 04 / 98 \\
12 / 04 / 98 \\
12 / 04 / 98 \\
12 / 04 / 98\end{array}$ & 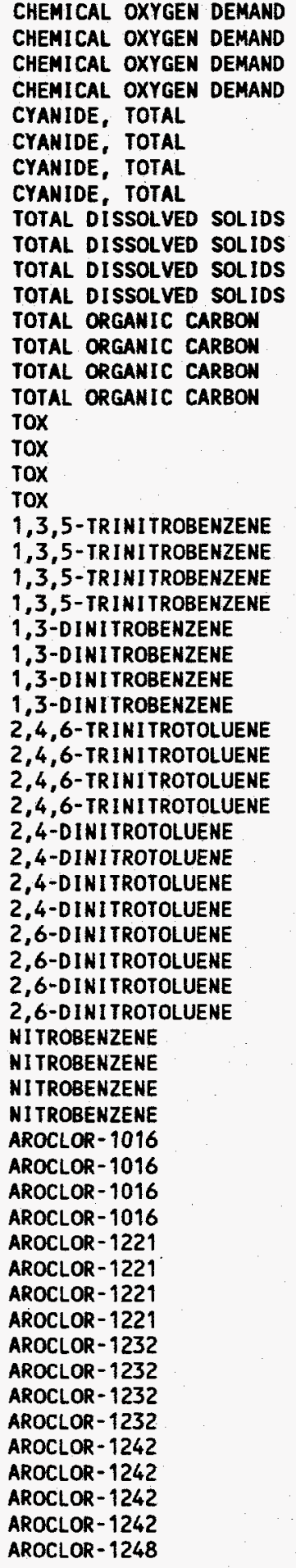 & 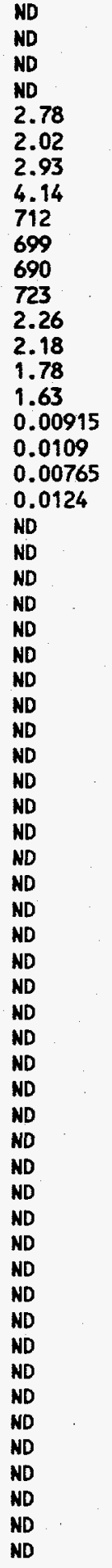 & $\begin{array}{l}5.00 \\
5.00 \\
5.00 \\
5.00 \\
1.30 \\
1.30 \\
1.30 \\
1.30 \\
5.00 \\
5.00 \\
5.00 \\
5.00 \\
1.00 \\
1.00 \\
1.00 \\
1.00 \\
0.005 \\
0.005 \\
0.005 \\
0.005 \\
0.030 \\
0.030 \\
0.030 \\
0.030 \\
0.090 \\
0.090 \\
0.090 \\
0.090 \\
0.030 \\
0.030 \\
0.030 \\
0.030 \\
0.030 \\
0.030 \\
0.030 \\
0.030 \\
0.010 \\
0.010 \\
0.010 \\
0.010 \\
0.030 \\
0.030 \\
0.030 \\
0.030 \\
1.0 \\
1.0 \\
1.0 \\
1.0 \\
1.0 \\
1.0 \\
1.0 \\
1.0 \\
1.0 \\
1.0 \\
1.0 \\
1.0 \\
1.0 \\
1.0 \\
1.0 \\
1.0 \\
1.0 \\
\end{array}$ & $\begin{array}{l}M G / L \\
M G / L \\
M G / L \\
M G / L \\
U G / L \\
U G / L \\
U G / L \\
U G / L \\
M G / L \\
M G / L \\
M G / L \\
M G / L \\
M G / L \\
M G / L \\
M G / L \\
U G / L \\
M G / L \\
M G / L \\
M G / L \\
M G / L \\
U G / L \\
U G / L \\
U G / L \\
U G / L \\
U G / L \\
U G / L \\
U G / L \\
U G / L \\
U G / L \\
U G / L \\
U G / L \\
U G / L \\
U G / L \\
U G / L \\
U G / L \\
U G / L \\
U G / L \\
U G / L \\
U G / L \\
U G / L \\
U G / L \\
U G / L \\
U G / L \\
U G / L \\
U G / L \\
U G / L \\
U G / L \\
U G / L \\
U G / L \\
U G / L \\
U G / L \\
U G / L \\
U G / L \\
U G / L \\
U G / L \\
U G / L \\
U G / L \\
U G / L \\
U G / L \\
U G / L \\
U G / L \\
\text { UG } \\
U\end{array}$ & $\begin{array}{l}\text { EPA } 410.4 \\
\text { EPA } 410.4 \\
\text { EPA } 410.4 \\
\text { EPA } 410.4 \\
\text { EPA CLP } \\
\text { EPA CLP } \\
\text { EPA CLP } \\
\text { EPA CLP } \\
\text { EPA } 160.1 \\
\text { EPA } 160.1 \\
\text { EPA } 160.1 \\
\text { EPA } 160.1 \\
\text { EPA } 415.1 \\
\text { EPA } 415.1 \\
\text { EPA } 415.1 \\
\text { EPA } 415.1 \\
\text { EPA } 9020 \\
\text { EPA } 9020 \\
\text { EPA } 9020 \\
\text { EPA } 9020 \\
\text { USATHAMA } \\
\text { USATHAMA } \\
\text { USATHAMA } \\
\text { USATHAMA } \\
\text { USATHAMA } \\
\text { USATHAMA } \\
\text { USATHAMA } \\
\text { USATHAMA } \\
\text { USATHAMA } \\
\text { USATHAMA } \\
\text { USATHAMA } \\
\text { USATHAMA } \\
\text { USATHAMA } \\
\text { USATHAMA } \\
\text { USATHAMA } \\
\text { USATHAMA } \\
\text { USATHAMA } \\
\text { USATHAMA } \\
\text { USATHAMA } \\
\text { USATHAMA } \\
\text { USATHAMA } \\
\text { USATHAMA } \\
\text { USATHAMA } \\
\text { USATHAMA } \\
\text { EPA } 8082 \\
\text { EPA } 8082 \\
\text { EPA } 8082 \\
\text { EPA } 8082 \\
\text { EPA } 8082 \\
\text { EPA } 8082 \\
\text { EPA } 8082 \\
\text { EPA } 8082 \\
\text { EPA } 8082 \\
\text { EPA } 8082 \\
\text { EPA } 8082 \\
\text { EPA } 8082 \\
\text { EPA } 8082 \\
\text { EPA } 8082 \\
\text { EPA } 8082 \\
\text { EPA } 8082 \\
\text { EPA } 8082\end{array}$ \\
\hline
\end{tabular}




\begin{tabular}{|c|c|c|c|c|c|c|}
\hline WSSRAP_ID & DATE_SAM & PARAMETER & CONC & DL & UNITS & METHOD \\
\hline 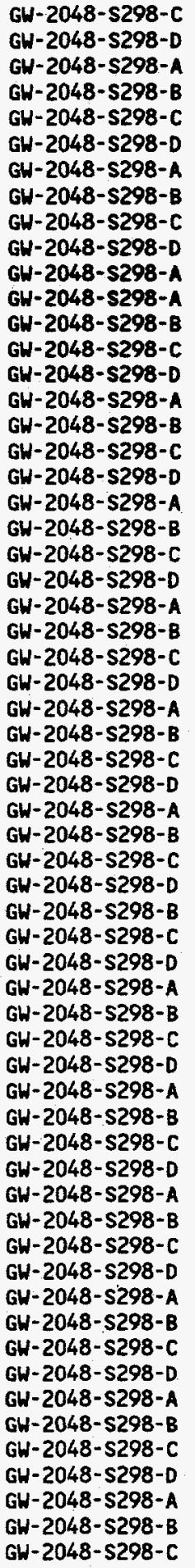 & $\begin{array}{l}12 / 04 / 98 \\
12 / 04 / 98 \\
12 / 04 / 98 \\
12 / 04 / 98 \\
12 / 04 / 98 \\
12 / 04 / 98 \\
12 / 04 / 98 \\
12 / 04 / 98 \\
12 / 04 / 98 \\
12 / 04 / 98 \\
12 / 04 / 98 \\
12 / 04 / 98 \\
12 / 04 / 98 \\
12 / 04 / 98 \\
12 / 04 / 98 \\
12 / 04 / 98 \\
12 / 04 / 98 \\
12 / 04 / 98 \\
12 / 04 / 98 \\
12 / 04 / 98 \\
12 / 04 / 98 \\
12 / 04 / 98 \\
12 / 04 / 98 \\
12 / 04 / 98 \\
12 / 04 / 98 \\
12 / 04 / 98 \\
12 / 04 / 98 \\
12 / 04 / 98 \\
12 / 04 / 98 \\
12 / 04 / 98 \\
12 / 04 / 98 \\
12 / 04 / 98 \\
12 / 04 / 98 \\
12 / 04 / 98 \\
12 / 04 / 98 \\
12 / 04 / 98 \\
12 / 04 / 98 \\
12 / 04 / 98 \\
12 / 04 / 98 \\
12 / 04 / 98 \\
12 / 04 / 98 \\
12 / 04 / 98 \\
12 / 04 / 98 \\
12 / 04 / 98 \\
12 / 04 / 98 \\
12 / 04 / 98 \\
12 / 04 / 98 \\
12 / 04 / 98 \\
12 / 04 / 98 \\
12 / 04 / 98 \\
12 / 04 / 98 \\
12 / 04 / 98 \\
12 / 04 / 98 \\
12 / 04 / 98 \\
12 / 04 / 98 \\
12 / 04 / 98 \\
12 / 04 / 98 \\
12 / 04 / 98 \\
12 / 04 / 98 \\
12 / 04 / 98 \\
12 / 04 / 98\end{array}$ & $\begin{array}{l}\text { AROCLOR-1248 } \\
\text { AROCLOR-1248 } \\
\text { AROCLOR-1248 } \\
\text { AROCLOR-1254 } \\
\text { AROCLOR-1254 } \\
\text { AROCLOR-1254 } \\
\text { AROCLOR-1254 } \\
\text { AROCLOR-1260 } \\
\text { AROCLOR-1260 } \\
\text { AROCLOR-1260 } \\
\text { AROCLOR-1260 } \\
\text { RADIUM-226 } \\
\text { RADIUH-226 } \\
\text { RADIUH-226 } \\
\text { RADIUM-226 } \\
\text { RADIUM-228 } \\
\text { RADIUM-228 } \\
\text { RADIUM-228 } \\
\text { RADIUM-228 } \\
\text { THORIUM-228 } \\
\text { THORIUM-228 } \\
\text { THORIUM-228 } \\
\text { THORIUM-228 } \\
\text { THORIUM-230 } \\
\text { THORIUM-230 } \\
\text { THORIUM-230 } \\
\text { THORIUM-230 } \\
\text { THORIUM-232 } \\
\text { THORIUM-232 } \\
\text { THORIUM-232 } \\
\text { THORIUM-232 } \\
\text { URANIUM, TOTAL } \\
\text { URANIUM, TOTAL } \\
\text { URANIUM, TOTAL } \\
\text { URANIUM, TOTAL } \\
\text { ACENAPHTHENE } \\
\text { ACENAPHTHENE } \\
\text { ACENAPHTHENE } \\
\text { ACENAPHTHENE } \\
\text { ACENAPHTHYLENE } \\
\text { ACENAPHTHYLENE } \\
\text { ACENAPHTHYLENE } \\
\text { ACENAPHTHYLENE } \\
\text { ANTHRACENE } \\
\text { ANTHRACENE } \\
\text { ANTHRACENE } \\
\text { ANTHRACENE } \\
\text { BENZO(A)ANTHRACENE } \\
\text { BENZO(A)ANTHRACENE } \\
\text { BENZO(A)ANTHRACENE } \\
\text { BENZO(A)ANTHRACENE } \\
\text { BENZO(A)PYRENE } \\
\text { BENZO(A)PYRENE } \\
\text { BENZO(A)PYRENE } \\
\text { BENZO(A)PYRENE } \\
\text { BENZO(B)FLUORANTHENE } \\
\text { BENZO(B) FLLUORANTHENE } \\
\text { BENZO(B)FLUORANTHENE } \\
\text { BENZO(B)FLUORANTHENE } \\
\text { BENZO(G, H,I) I)ERYLENE } \\
\text { BENZO(G,H,I)PERYLENE }\end{array}$ & 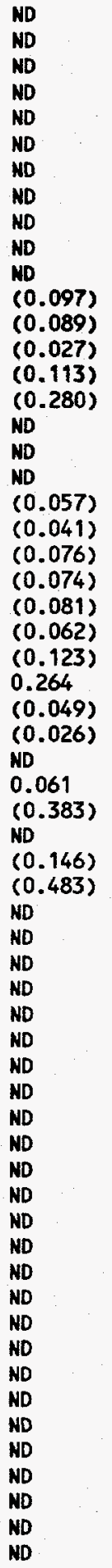 & $\begin{array}{l}1.0 \\
1.0 \\
1.0 \\
1.0 \\
1.0 \\
1.0 \\
1.0 \\
1.0 \\
1.0 \\
1.0 \\
1.0 \\
0.147 \\
0.199 \\
0.196 \\
0.209 \\
0.469 \\
0.469 \\
0.469 \\
0.469 \\
0.091 \\
0.089 \\
0.105 \\
0.171 \\
0.261 \\
0.40 \\
0.142 \\
0.050 \\
0.072 \\
0.082 \\
0.208 \\
0.050 \\
0.677 \\
0.677 \\
0.677 \\
0.677 \\
5.0 \\
5.0 \\
5.0 \\
5.0 \\
5.0 \\
5.0 \\
5.0 \\
5.0 \\
5.0 \\
5.0 \\
5.0 \\
5.0 \\
5.0 \\
5.0 \\
5.0 \\
5.0 \\
5.0 \\
5.0 \\
5.0 \\
5.0 \\
5.0 \\
5.0 \\
5.0 \\
5.0 \\
5.0 \\
5.0 \\
\end{array}$ & $\begin{array}{l}U G / L \\
U G / L \\
U G / L \\
U G / L \\
U G / L \\
U G / L \\
U G / L \\
U G / L \\
U G / L \\
U G / L \\
U G / L \\
P C I / L \\
P C I / L \\
P C I / L \\
P C I / L \\
P C I / L \\
P C I / L \\
P C I / L \\
P C I / L \\
P C I / L \\
P C I / L \\
P C I / L \\
P C I / L \\
P C I / L \\
P C I / L \\
P C I / L \\
P C I / L \\
P C I / L \\
P C I / L \\
P C I / L \\
P C I / L \\
P C I / L \\
P C I / L \\
P C I / L \\
P C I / L \\
U G / L \\
U G / L \\
U G / L \\
U G / L \\
U G / L \\
U G / L \\
U G / L \\
U G / L \\
U G / L \\
U G / L \\
U G / L \\
U G / L \\
U G / L \\
U G / L \\
U G / L \\
U G / L \\
U G / L \\
U G / L \\
U G / L \\
U G / L \\
U G / L \\
U G / L \\
U G / L \\
\text { UG/L }\end{array}$ & $\begin{array}{l}\text { EPA } 8082 \\
\text { EPA } 8082 \\
\text { EPA } 8082 \\
\text { EPA } 8082 \\
\text { EPA } 8082 \\
\text { EPA } 8082 \\
\text { EPA } 8082 \\
\text { EPA } 8082 \\
\text { EPA } 8082 \\
\text { EPA } 8082 \\
\text { EPA } 8082 \\
\text { SM-705M } \\
\text { SM-705M } \\
\text { SM-705M } \\
\text { SM-705M } \\
\text { EPA } 9320 \\
\text { EPA } 9320 \\
\text { EPA } 9320 \\
\text { EPA } 9320 \\
\text { LANL ER200 } \\
\text { LANL ER200 } \\
\text { LANL ER200 } \\
\text { LANL ER200 } \\
\text { LANL ER200 } \\
\text { LANL ER200 } \\
\text { LANL ER200 } \\
\text { LANL ER200 } \\
\text { LANL ER200 } \\
\text { LANL ER200 } \\
\text { LANL ER200 } \\
\text { LANL ER200 } \\
\text { ASTM } 5174-91 \\
\text { ASTM } 5174-91 \\
\text { ASTM } 5174-91 \\
\text { ASTM } 5174-91 \\
\text { EPA } 8310 \\
\text { EPA } 8310 \\
\text { EPA } 8310 \\
\text { EPA } 8310 \\
\text { EPA } 8310 \\
\text { EPA } 8310 \\
\text { EPA } 8310 \\
\text { EPA } 8310 \\
\text { EPA } 8310 \\
\text { EPA } 8310 \\
\text { EPA } 8310 \\
\text { EPA } 8310 \\
\text { EPA } 8310 \\
\text { EPA } 8310 \\
\text { EPA } 8310 \\
\text { EPA } 8310 \\
\text { EPA } 8310 \\
\text { EPA } 8310 \\
\text { EPA } 8310 \\
\text { EPA } 8310 \\
\text { EPA } 8310 \\
\text { EPA } 8310 \\
\text { EPA } 8310 \\
\text { EPA } 8310 \\
\text { EPA } 8310 \\
\text { EPA } 8310 \\
\end{array}$ \\
\hline
\end{tabular}




\begin{tabular}{|c|c|c|c|c|c|c|}
\hline USSRAP_ID & DATE_SAM & PARAMETER & CONC & $\mathrm{DL}$ & UNITS & METHOD \\
\hline 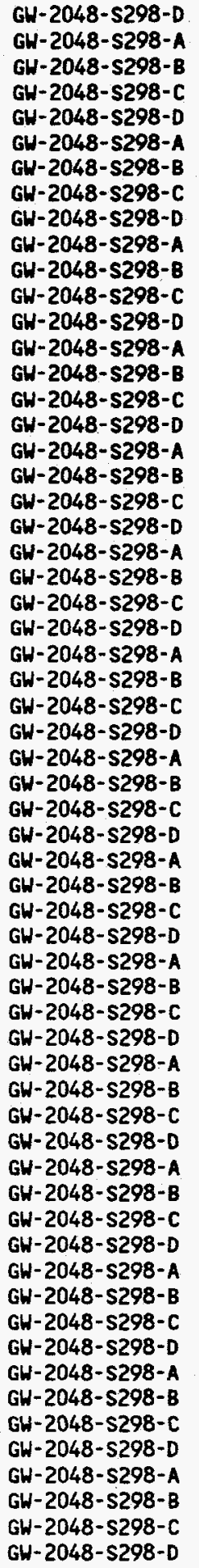 & $\begin{array}{l}12 / 04 / 98 \\
12 / 04 / 98 \\
12 / 04 / 98 \\
12 / 04 / 98 \\
12 / 04 / 98 \\
12 / 04 / 98 \\
12 / 04 / 98 \\
12 / 04 / 98 \\
12 / 04 / 98 \\
12 / 04 / 98 \\
12 / 04 / 98 \\
12 / 04 / 98 \\
12 / 04 / 98 \\
12 / 04 / 98 \\
12 / 04 / 98 \\
12 / 04 / 98 \\
12 / 04 / 98 \\
12 / 04 / 98 \\
12 / 04 / 98 \\
12 / 04 / 98 \\
12 / 04 / 98 \\
12 / 04 / 98 \\
12 / 04 / 98 \\
12 / 04 / 98 \\
12 / 04 / 98 \\
12 / 04 / 98 \\
12 / 04 / 98 \\
12 / 04 / 98 \\
12 / 04 / 98 \\
12 / 04 / 98 \\
12 / 04 / 98 \\
12 / 04 / 98 \\
12 / 04 / 98 \\
12 / 04 / 98 \\
12 / 04 / 98 \\
12 / 04 / 98 \\
12 / 04 / 98 \\
12 / 04 / 98 \\
12 / 04 / 98 \\
12 / 04 / 98 \\
12 / 04 / 98 \\
12 / 04 / 98 \\
12 / 04 / 98 \\
12 / 04 / 98 \\
12 / 04 / 98 \\
12 / 04 / 98 \\
12 / 04 / 98 \\
12 / 04 / 98 \\
12 / 04 / 98 \\
12 / 04 / 98 \\
12 / 04 / 98 \\
12 / 04 / 98 \\
12 / 04 / 98 \\
12 / 04 / 98 \\
12 / 04 / 98 \\
12 / 04 / 98 \\
12 / 04 / 98 \\
12 / 04 / 98 \\
12 / 04 / 98 \\
12 / 04 / 98 \\
12 / 04 / 98\end{array}$ & 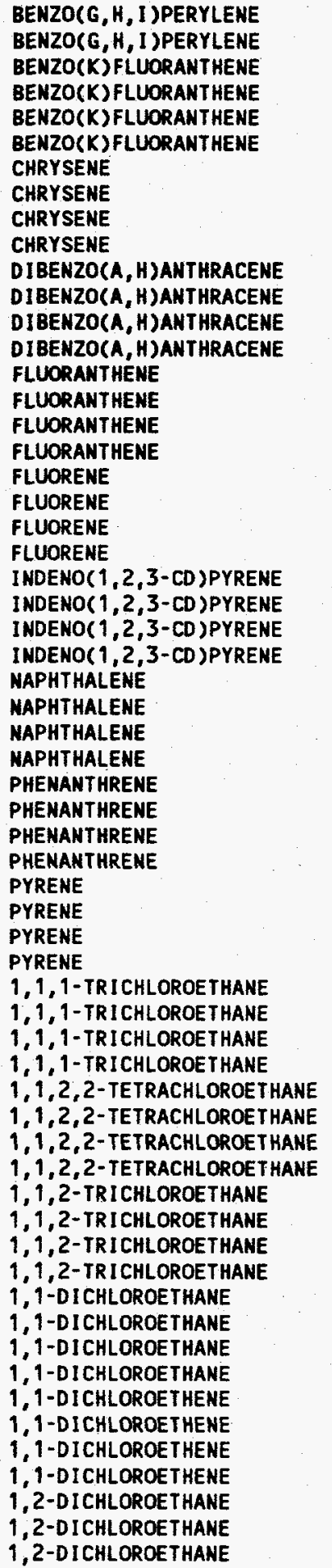 & $\begin{array}{l}\text { ND } \\
\text { ND } \\
\text { ND } \\
\text { ND } \\
\text { ND } \\
\text { ND } \\
\text { ND } \\
\text { ND } \\
\text { ND } \\
\text { ND } \\
\text { ND } \\
\text { ND } \\
\text { ND } \\
\text { ND } \\
\text { ND } \\
\text { ND } \\
\text { ND } \\
\text { ND } \\
\text { ND } \\
\text { ND } \\
\text { ND } \\
\text { ND } \\
\text { ND } \\
\text { ND } \\
\text { ND } \\
\text { ND } \\
\text { ND } \\
\text { ND } \\
\text { ND } \\
\text { ND } \\
\text { ND } \\
\text { ND } \\
\text { ND } \\
\text { ND } \\
\text { ND } \\
\text { ND } \\
\text { ND } \\
\text { ND } \\
\text { ND } \\
\text { ND } \\
\text { ND } \\
\text { ND } \\
\text { ND } \\
\text { ND } \\
\text { ND } \\
\text { ND } \\
\text { ND } \\
\text { ND } \\
\text { ND } \\
\text { ND } \\
\text { ND } \\
\text { ND } \\
\text { ND } \\
\text { ND } \\
\text { ND } \\
\text { ND } \\
\text { ND } \\
\text { ND } \\
\text { ND } \\
\text { ND } \\
\text { ND }\end{array}$ & $\begin{array}{l}5.0 \\
5.0 \\
5.0 \\
5.0 \\
5.0 \\
5.0 \\
5.0 \\
5.0 \\
5.0 \\
5.0 \\
5.0 \\
5.0 \\
5.0 \\
5.0 \\
5.0 \\
5.0 \\
5.0 \\
5.0 \\
5.0 \\
5.0 \\
5.0 \\
5.0 \\
5.0 \\
5.0 \\
5.0 \\
5.0 \\
5.0 \\
5.0 \\
5.0 \\
5.0 \\
5.0 \\
5.0 \\
5.0 \\
5.0 \\
5.0 \\
5.0 \\
5.0 \\
5.0 \\
10 \\
10 \\
10 \\
10 \\
10 \\
10 \\
10 \\
10 \\
10 \\
10 \\
10 \\
10 \\
10 \\
10 \\
10 \\
10 \\
10 \\
10 \\
10 \\
10 \\
10 \\
10 \\
10\end{array}$ & $\begin{array}{l}\text { UG/L } \\
U G / L \\
U G / L \\
U G / L \\
U G / L \\
U G / L \\
U G / L \\
U G / L \\
U G / L \\
U G / L \\
U G / L \\
U G / L \\
U G / L \\
U G / L \\
U G / L \\
U G / L \\
U G / L \\
U G / L \\
U G / L \\
U G / L \\
U G / L \\
U G / L \\
U G / L \\
U G / L \\
U G / L \\
U G / L \\
U G / L \\
U G / L \\
U G / L \\
U G / L \\
U G / L \\
U G / L \\
U G / L \\
U G / L \\
U G / L \\
U G / L \\
U G / L \\
U G / L \\
U G / L \\
U G / L \\
U G / L \\
U G / L \\
U G / L \\
U G / L \\
U G / L \\
U G / L \\
U G / L \\
U G / L \\
U G / L \\
U G / L \\
U G / L \\
U G / L \\
U G / L \\
U G / L \\
U G / L \\
U G / L \\
U G / L \\
U G / L \\
U G / L \\
U G / L \\
U G / L \\
\text { U. }\end{array}$ & $\begin{array}{l}\text { EPA } 8310 \\
\text { EPA } 8310 \\
\text { EPA } 8310 \\
\text { EPA } 8310 \\
\text { EPA } 8310 \\
\text { EPA } 8310 \\
\text { EPA } 8310 \\
\text { EPA } 8310 \\
\text { EPA } 8310 \\
\text { EPA } 8310 \\
\text { EPA } 8310 \\
\text { EPA } 8310 \\
\text { EPA } 8310 \\
\text { EPA } 8310 \\
\text { EPA } 8310 \\
\text { EPA } 8310 \\
\text { EPA } 8310 \\
\text { EPA } 8310 \\
\text { EPA } 8310 \\
\text { EPA } 8310 \\
\text { EPA } 8310 \\
\text { EPA } 8310 \\
\text { EPA } 8310 \\
\text { EPA } 8310 \\
\text { EPA } 8310 \\
\text { EPA } 8310 \\
\text { EPA } 8310 \\
\text { EPA } 8310 \\
\text { EPA } 8310 \\
\text { EPA } 8310 \\
\text { EPA } 8310 \\
\text { EPA } 8310 \\
\text { EPA } 8310 \\
\text { EPA } 8310 \\
\text { EPA } 8310 \\
\text { EPA } 8310 \\
\text { EPA } 8310 \\
\text { EPA } 8310 \\
\text { EPA CLP } \\
\text { EPA CLP } \\
\text { EPA CLP } \\
\text { EPA CLP } \\
\text { EPA CLP } \\
\text { EPA CLP } \\
\text { EPA CLP } \\
\text { EPA CLP } \\
\text { EPA CLP } \\
\text { EPA } C L P \\
\text { EPA CLP } \\
\text { EPA } C L P \\
\text { EPA CLP } \\
\text { EPA CLP } \\
\text { EPA CLP } \\
\text { EPA CLP } \\
\text { EPA CLP } \\
\text { EPA CLP } \\
\text { EPA CLP } \\
\text { EPA CLP } \\
\text { EPA } C L P \\
\text { EPA CLP } \\
\text { EPA CLP }\end{array}$ \\
\hline
\end{tabular}




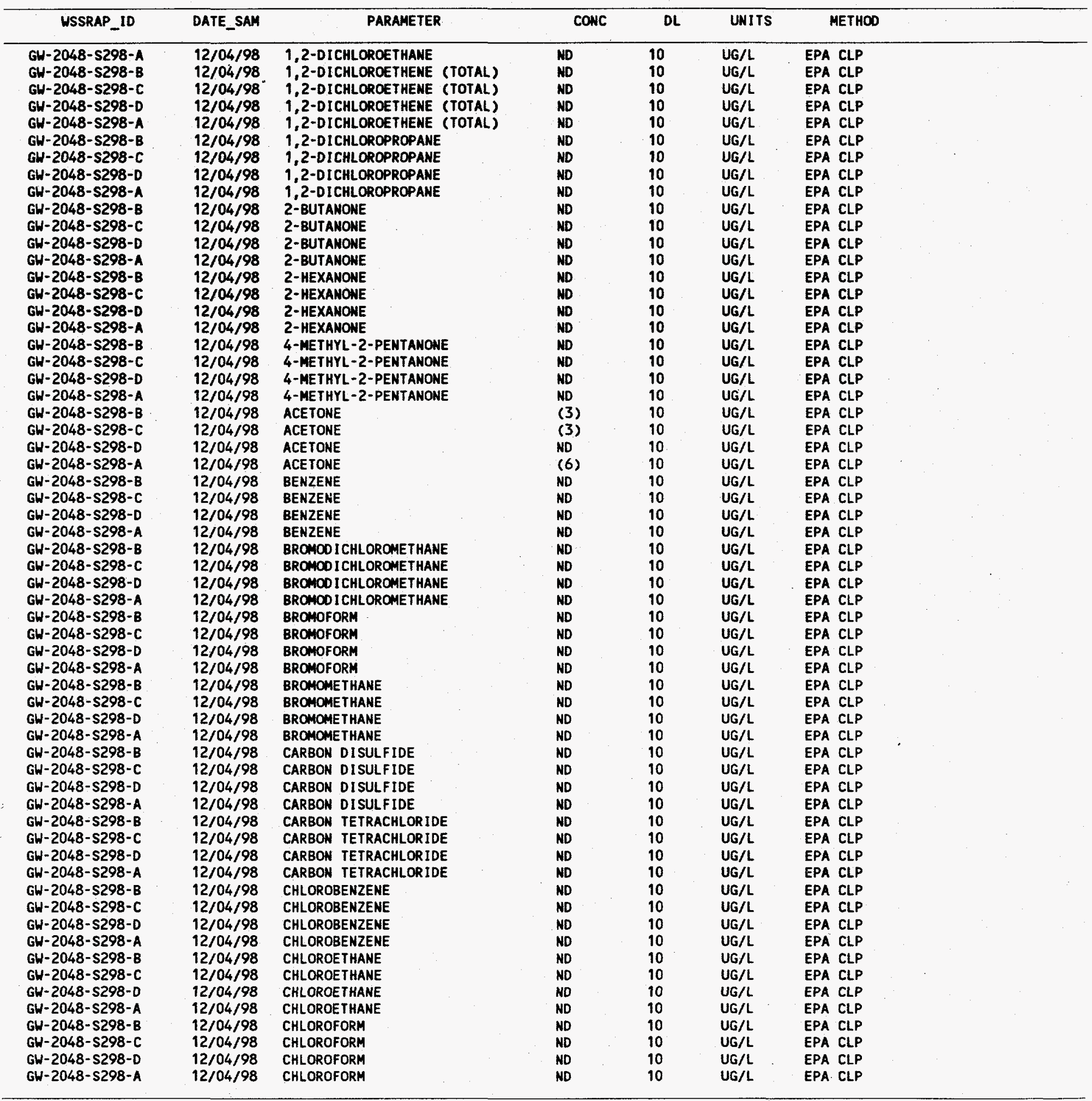




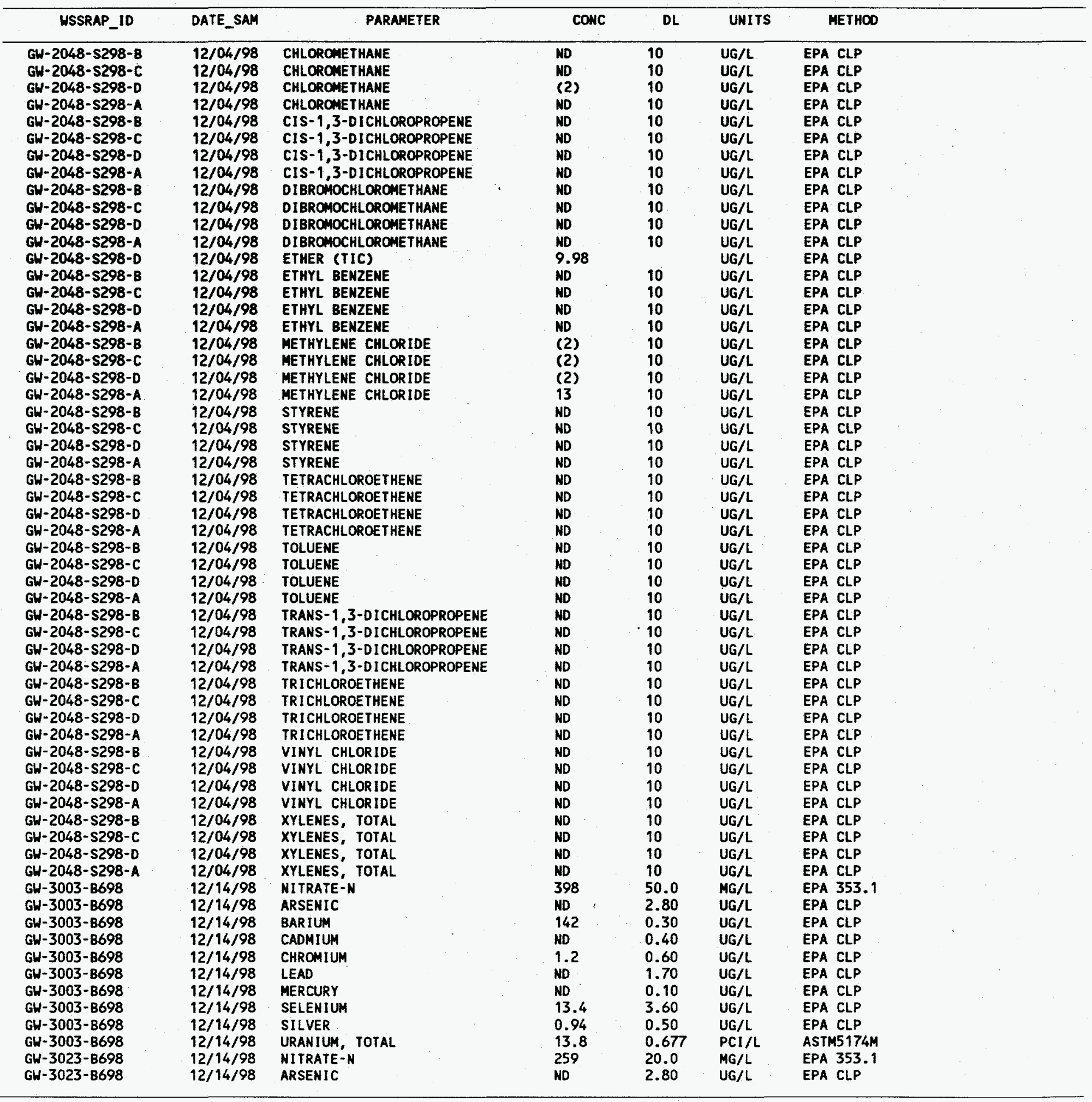




\begin{tabular}{|c|c|c|c|c|c|c|c|}
\hline USSRAP_ID & DATE_SAM & PARAMETER & CONC & DL & UNITS & METHOD & \\
\hline 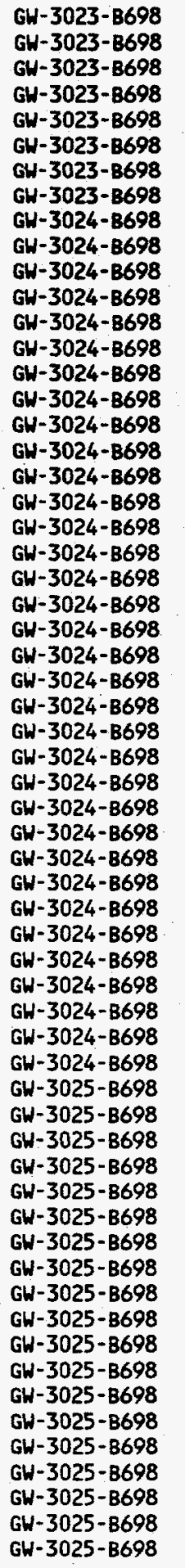 & $\begin{array}{l}12 / 14 / 98 \\
12 / 14 / 98 \\
12 / 14 / 98 \\
12 / 14 / 98 \\
12 / 14 / 98 \\
12 / 14 / 98 \\
12 / 14 / 98 \\
12 / 14 / 98 \\
12 / 15 / 98 \\
12 / 15 / 98 \\
12 / 15 / 98 \\
12 / 15 / 98 \\
12 / 15 / 98 \\
12 / 15 / 98 \\
12 / 15 / 98 \\
12 / 15 / 98 \\
12 / 15 / 98 \\
12 / 15 / 98 \\
12 / 15 / 98 \\
12 / 15 / 98 \\
12 / 15 / 98 \\
12 / 15 / 98 \\
12 / 15 / 98 \\
12 / 15 / 98 \\
12 / 15 / 98 \\
12 / 15 / 98 \\
12 / 15 / 98 \\
12 / 15 / 98 \\
12 / 15 / 98 \\
12 / 15 / 98 \\
12 / 15 / 98 \\
12 / 15 / 98 \\
12 / 15 / 98 \\
12 / 15 / 98 \\
12 / 15 / 98 \\
12 / 15 / 98 \\
12 / 15 / 98 \\
12 / 15 / 98 \\
12 / 15 / 98 \\
12 / 15 / 98 \\
12 / 15 / 98 \\
12 / 15 / 98 \\
12 / 15 / 98 \\
12 / 15 / 98 \\
12 / 15 / 98 \\
12 / 15 / 98 \\
12 / 15 / 98 \\
12 / 15 / 98 \\
12 / 15 / 98 \\
12 / 15 / 98 \\
12 / 15 / 98 \\
12 / 15 / 98 \\
12 / 15 / 98 \\
12 / 15 / 98 \\
12 / 15 / 98 \\
12 / 15 / 98 \\
12 / 15 / 98 \\
12 / 15 / 98 \\
12 / 15 / 98 \\
12 / 15 / 98 \\
12 / 15 / 98\end{array}$ & 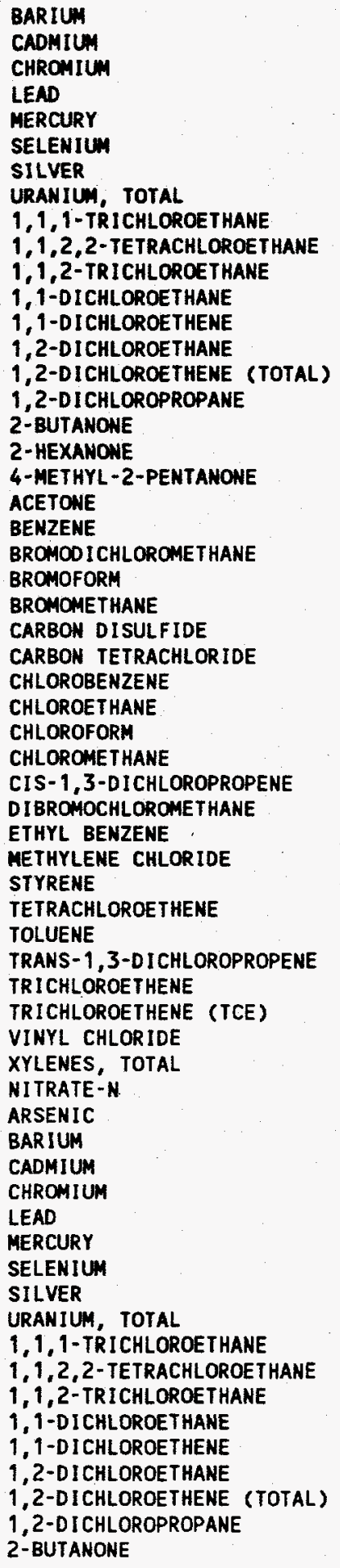 & $\begin{array}{l}45.1 \\
\text { ND } \\
4.7 \\
4.4 \\
0.42 \\
11.1 \\
\text { ND } \\
5.90 \\
\text { ND } \\
\text { ND } \\
\text { ND } \\
\text { ND } \\
\text { ND } \\
\text { ND } \\
\text { ND } \\
\text { ND } \\
\text { ND } \\
\text { ND } \\
\text { ND } \\
\text { ND } \\
\text { (2) } \\
\text { ND } \\
\text { ND } \\
\text { ND } \\
\text { ND } \\
\text { ND } \\
\text { ND } \\
\text { ND } \\
\text { ND } \\
\text { ND } \\
\text { ND } \\
\text { ND } \\
\text { ND } \\
\text { (2) } \\
\text { ND } \\
\text { ND } \\
\text { (5) } \\
\text { ND } \\
\text { ND } \\
\text { ND } \\
\text { ND } \\
\text { (6) } \\
380 \\
\text { ND } \\
243 \\
\text { ND } \\
1.6 \\
\text { ND } \\
\text { ND } \\
10.2 \\
\text { ND } \\
3.15 \\
\text { ND } \\
\text { ND } \\
\text { ND } \\
\text { ND } \\
\text { ND } \\
\text { ND } \\
\text { ND } \\
\text { ND } \\
\text { ND }\end{array}$ & $\begin{array}{l}0.30 \\
0.40 \\
0.60 \\
1.70 \\
0.10 \\
3.60 \\
0.50 \\
0.677 \\
10 \\
10 \\
10 \\
10 \\
10 \\
10 \\
10 \\
10 \\
10 \\
10 \\
10 \\
10 \\
10 \\
10 \\
10 \\
10 \\
10 \\
10 \\
10 \\
10 \\
10 \\
10 \\
10 \\
10 \\
10 \\
10 \\
10 \\
10 \\
10 \\
10 \\
10 \\
1.00 \\
10 \\
10 \\
50.0 \\
2.80 \\
0.30 \\
0.40 \\
0.60 \\
1.70 \\
0.10 \\
3.60 \\
0.50 \\
0.064 \\
10 \\
10 \\
10 \\
10 \\
10 \\
10 \\
10 \\
10 \\
10 \\
10 \\
10 \\
10 \\
0\end{array}$ & $\begin{array}{l}\text { UG/L } \\
U G / L \\
\text { UG/L } \\
U G / L \\
U G / L \\
U G / L \\
U G / L \\
P C I / L \\
U G / L \\
U G / L \\
U G / L \\
U G / L \\
U G / L \\
U G / L \\
U G / L \\
U G / L \\
U G / L \\
U G / L \\
U G / L \\
U G / L \\
U G / L \\
U G / L \\
U G / L \\
U G / L \\
U G / L \\
U G / L \\
U G / L \\
U G / L \\
U G / L \\
U G / L \\
U G / L \\
U G / L \\
U G / L \\
U G / L \\
U G / L \\
U G / L \\
U G / L \\
U G / L \\
U G / L \\
U G / L \\
U G / L \\
U G / L \\
M G / L \\
U G / L \\
U G / L \\
U G / L \\
U G / L \\
U G / L \\
U G / L \\
U G / L \\
U G / L \\
P C I / L \\
U G / L \\
U G / L \\
U G / L \\
U G / L \\
U G / L \\
U G / L \\
U G / L \\
U G / L \\
U G / L \\
\text { US }\end{array}$ & $\begin{array}{l}\text { EPA CLP } \\
\text { EPA CLP } \\
\text { EPA CLP } \\
\text { EPA CLP } \\
\text { EPA CLP } \\
\text { EPA CLP } \\
\text { EPA CLP } \\
\text { ASTM5174M } \\
\text { EPA CLP } \\
\text { EPA CLP } \\
\text { EPA CLP } \\
\text { EPA CLP } \\
\text { EPA CLP } \\
\text { EPA CLP } \\
\text { EPA CLP } \\
\text { EPA CLP } \\
\text { EPA CLP } \\
\text { EPA CLP } \\
\text { EPA CLP } \\
\text { EPA CLP } \\
\text { EPA CLP } \\
\text { EPA CLP } \\
\text { EPA CLP } \\
\text { EPA CLP } \\
\text { EPA CLP } \\
\text { EPA CLP } \\
\text { EPA CLP } \\
\text { EPA CLP } \\
\text { EPA CLP } \\
\text { EPA CLP } \\
\text { EPA CLP } \\
\text { EPA CLP } \\
\text { EPA CLP } \\
\text { EPA CLP } \\
\text { EPA CLP } \\
\text { EPA CLP } \\
\text { EPA CLP } \\
\text { EPA CLP } \\
\text { EPA CLP } \\
\text { EPA } 8021 A \\
\text { EPA CLP } \\
\text { EPA CLP } \\
\text { EPA } 353.1 \\
\text { EPA CLP } \\
\text { EPA CLP } \\
\text { EPA CLP } \\
\text { EPA CLP } \\
\text { EPA CLP } \\
\text { EPA CLP } \\
\text { EPA CLP } \\
\text { EPA CLP } \\
\text { ASTM } 5174-91 \\
\text { EPA CLP } \\
\text { EPA CLP } \\
\text { EPA CLP } \\
\text { EPA CLP } \\
\text { EPA CLP } \\
\text { EPA CLP } \\
\text { EPA CLP } \\
\text { EPA CLP } \\
\text { EPA CLP } \\
\end{array}$ & - \\
\hline
\end{tabular}




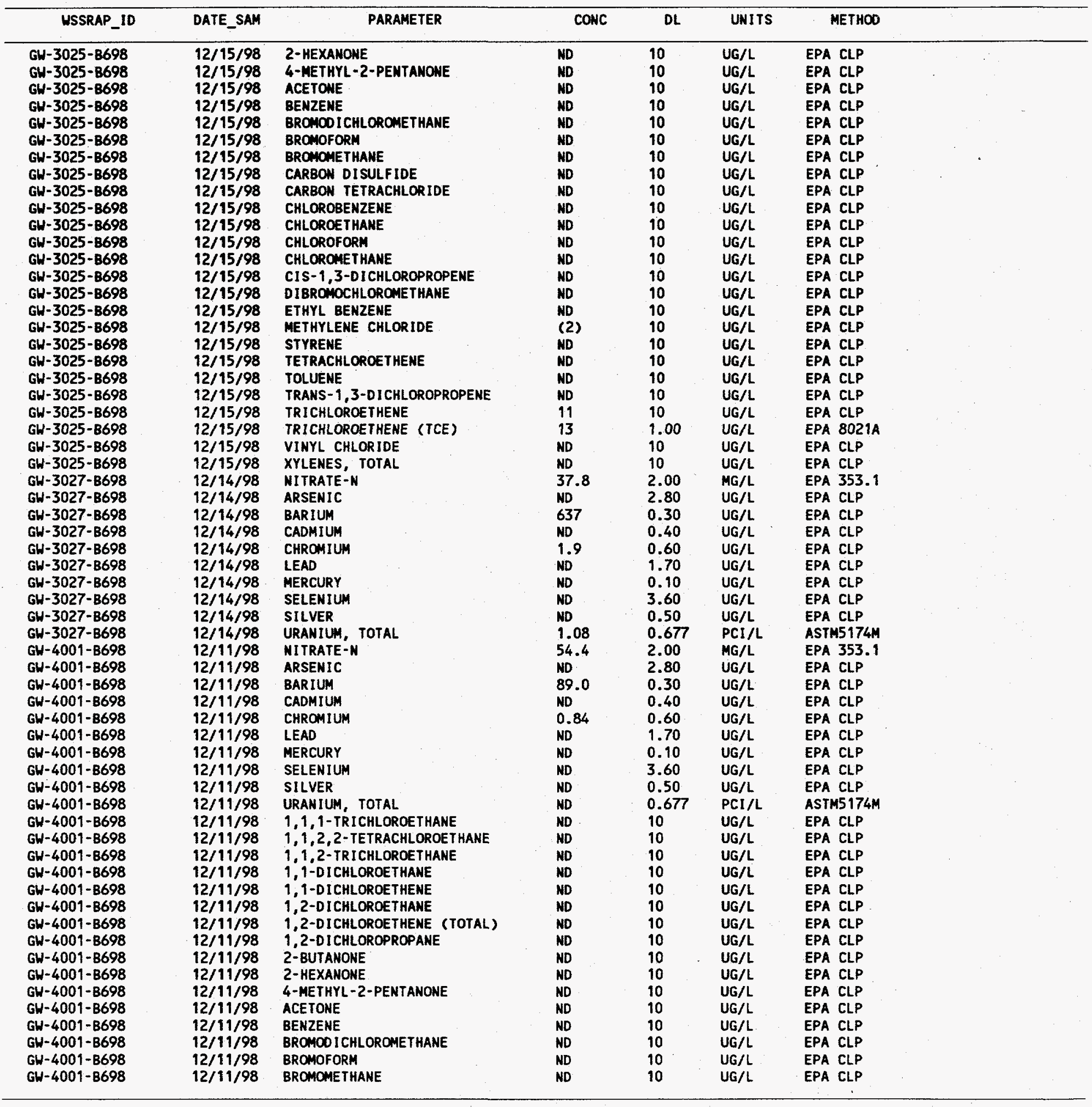




\begin{tabular}{|c|c|c|c|c|c|c|}
\hline USSRAP_10 & DATE_SAM & PARAMETER & CONC & DL & UNITS & METHOD \\
\hline 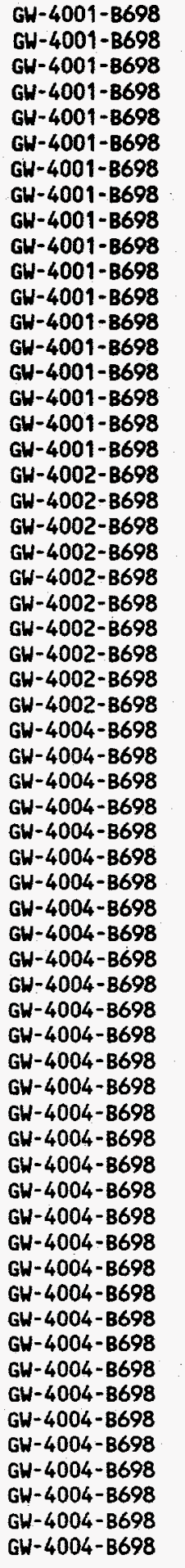 & $\begin{array}{l}12 / 11 / 98 \\
12 / 11 / 98 \\
12 / 11 / 98 \\
12 / 11 / 98 \\
12 / 11 / 98 \\
12 / 11 / 98 \\
12 / 11 / 98 \\
12 / 11 / 98 \\
12 / 11 / 98 \\
12 / 11 / 98 \\
12 / 11 / 98 \\
12 / 11 / 98 \\
12 / 11 / 98 \\
12 / 11 / 98 \\
12 / 11 / 98 \\
12 / 11 / 98 \\
12 / 11 / 98 \\
12 / 11 / 98 \\
12 / 11 / 98 \\
12 / 11 / 98 \\
12 / 11 / 98 \\
12 / 11 / 98 \\
12 / 11 / 98 \\
12 / 11 / 98 \\
12 / 11 / 98 \\
12 / 11 / 98 \\
12 / 11 / 98 \\
12 / 11 / 98 \\
12 / 11 / 98 \\
12 / 11 / 98 \\
12 / 11 / 98 \\
12 / 11 / 98 \\
12 / 11 / 98 \\
12 / 11 / 98 \\
12 / 11 / 98 \\
12 / 11 / 98 \\
12 / 11 / 98 \\
12 / 11 / 98 \\
12 / 11 / 98 \\
12 / 11 / 98 \\
12 / 11 / 98 \\
12 / 11 / 98 \\
12 / 11 / 98 \\
12 / 11 / 98 \\
12 / 11 / 98 \\
12 / 11 / 98 \\
12 / 11 / 98 \\
12 / 11 / 98 \\
12 / 11 / 98 \\
12 / 11 / 98 \\
12 / 11 / 98 \\
12 / 11 / 98 \\
12 / 11 / 98 \\
12 / 11 / 98 \\
12 / 11 / 98 \\
12 / 111 / 98 \\
12 / 111 / 98 \\
12 / 11 / 98 \\
12 / 11 / 98 \\
12 / 11 / 98 \\
12 / 111 / 98\end{array}$ & 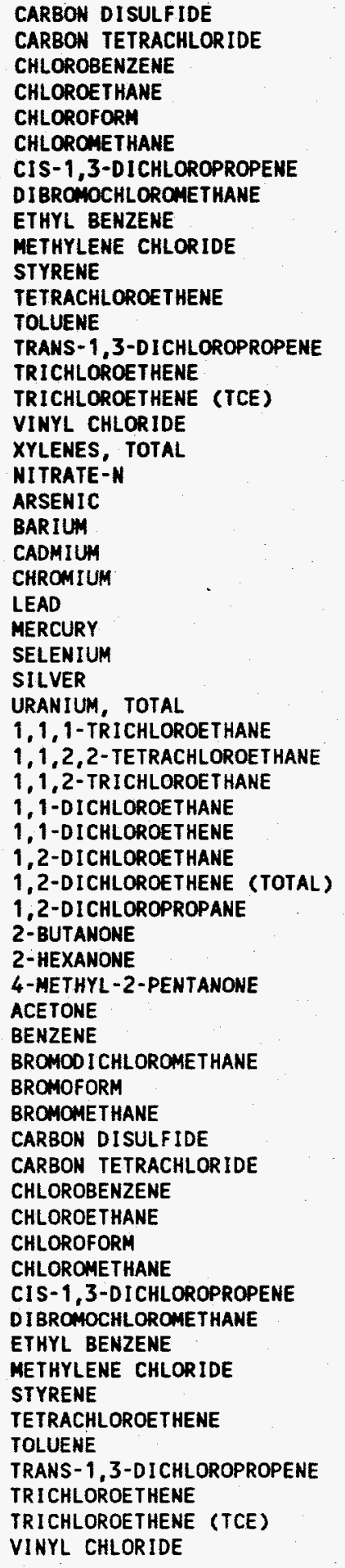 & $\begin{array}{l}\text { ND } \\
\text { ND } \\
\text { ND } \\
\text { ND } \\
\text { ND } \\
\text { ND } \\
\text { ND } \\
\text { ND } \\
\text { ND } \\
\text { (2) } \\
\text { ND } \\
\text { ND } \\
\text { ND } \\
\text { ND } \\
\text { (5) } \\
4.8 \\
\text { ND } \\
\text { ND } \\
\text { O.85 } \\
\text { ND } \\
\text { 154 } \\
\text { ND } \\
\text { 20.6 } \\
25.0 \\
\text { ND } \\
4.9 \\
\text { ND } \\
\text { ND } \\
\text { ND } \\
\text { ND } \\
\text { ND } \\
\text { ND } \\
\text { ND } \\
\text { ND } \\
\text { ND } \\
\text { ND } \\
\text { ND } \\
\text { ND } \\
\text { ND } \\
\text { ND } \\
\text { ND } \\
\text { ND } \\
\text { ND } \\
\text { ND } \\
\text { ND } \\
\text { ND } \\
\text { ND } \\
\text { ND } \\
\text { ND } \\
\text { ND } \\
\text { ND } \\
\text { ND } \\
\text { ND } \\
\text { (2) } \\
\text { ND } \\
\text { ND } \\
\text { ND } \\
\text { ND } \\
\text { ND } \\
\text { ND } \\
\text { ND }\end{array}$ & $\begin{array}{l}10 \\
10 \\
10 \\
10 \\
10 \\
10 \\
10 \\
10 \\
10 \\
10 \\
10 \\
10 \\
10 \\
10 \\
10 \\
1.00 \\
10 \\
10 \\
0.02 \\
2.80 \\
0.30 \\
0.40 \\
0.60 \\
1.70 \\
0.10 \\
3.60 \\
0.50 \\
0.677 \\
10 \\
10 \\
10 \\
10 \\
10 \\
10 \\
10 \\
10 \\
10 \\
10 \\
10 \\
10 \\
10 \\
10 \\
10 \\
10 \\
10 \\
10 \\
10 \\
10 \\
10 \\
10 \\
10 \\
10 \\
10 \\
10 \\
10 \\
10 \\
10 \\
10 \\
10 \\
1.00 \\
10 \\
10 \\
10 \\
10 \\
10 \\
10 \\
10 \\
10 \\
10 \\
0\end{array}$ & 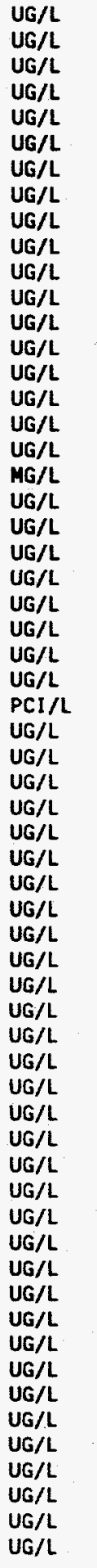 & 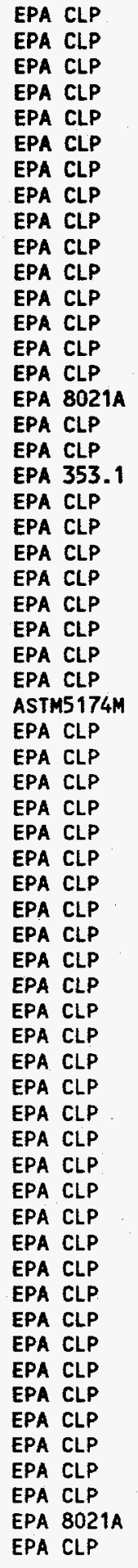 \\
\hline
\end{tabular}




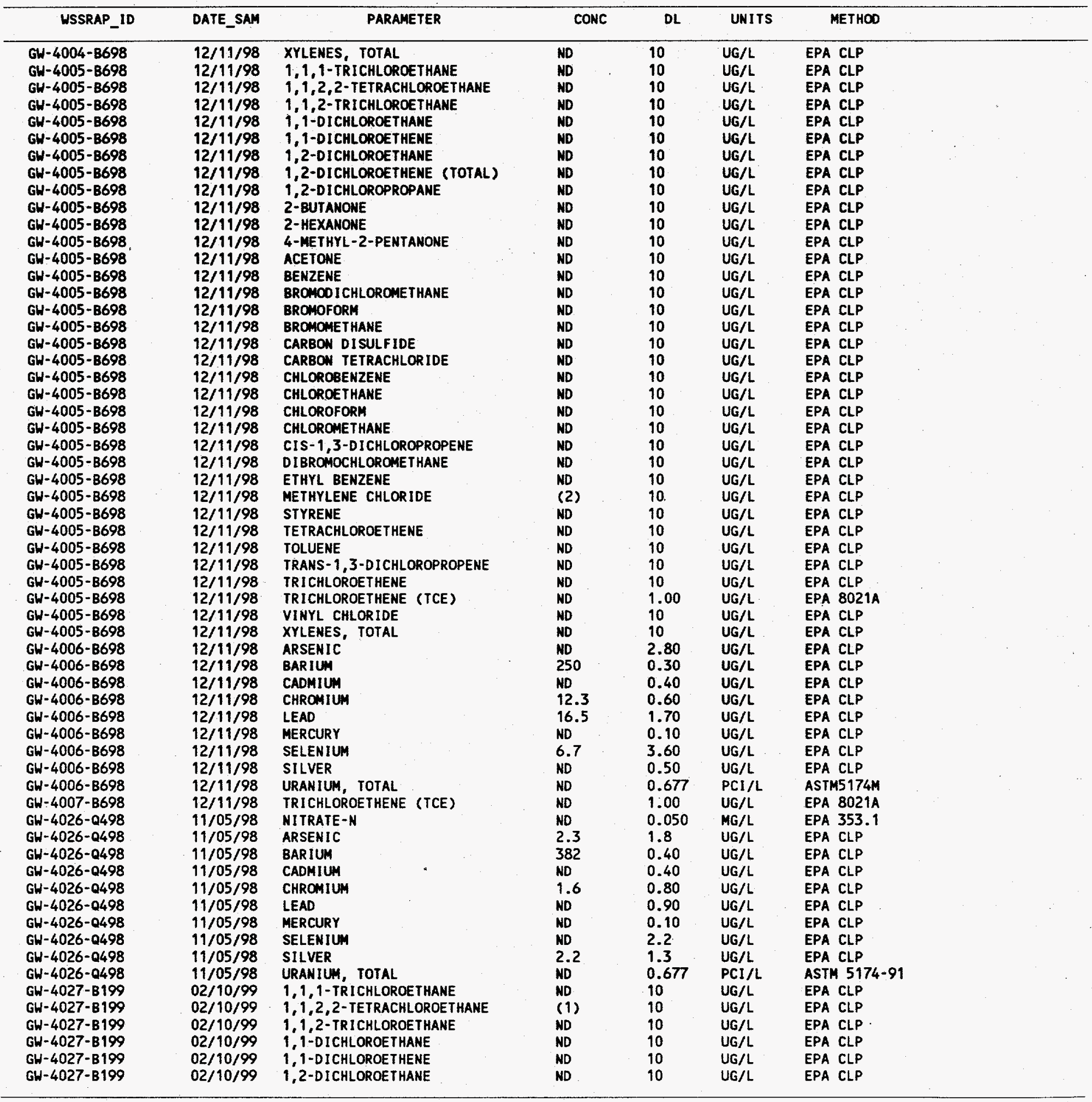




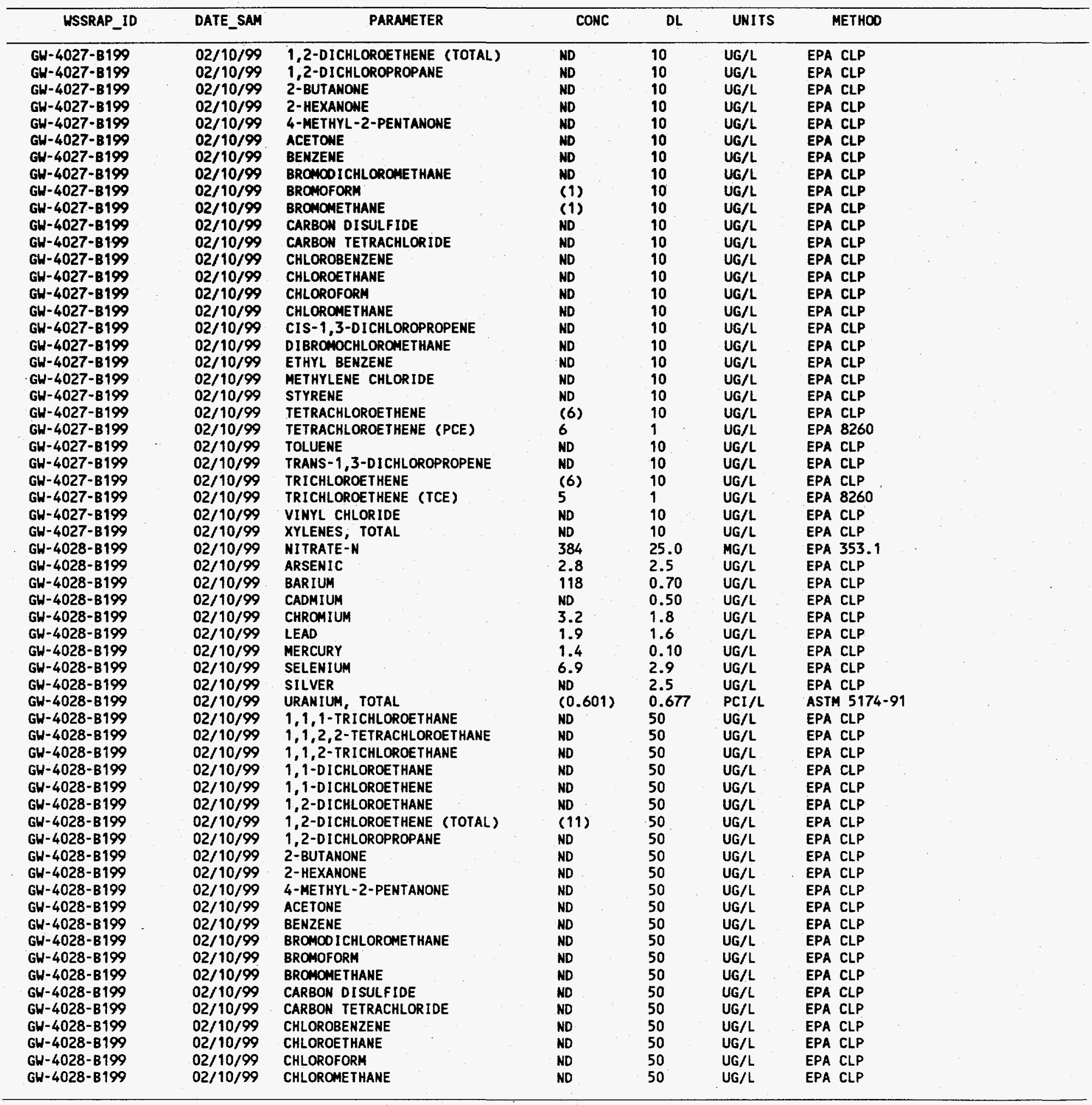




\begin{tabular}{|c|c|c|c|c|c|c|c|}
\hline USSRAP_ID & DATE_SAM & PARAMETER & CONC & $\mathrm{DL}$ & UNITS & METHOD & \\
\hline 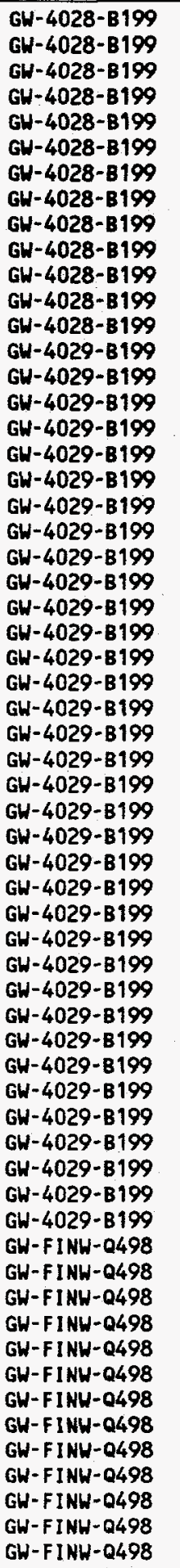 & $\begin{array}{l}02 / 10 / 99 \\
02 / 10 / 99 \\
02 / 10 / 99 \\
02 / 10 / 99 \\
02 / 10 / 99 \\
02 / 10 / 99 \\
02 / 10 / 99 \\
02 / 10 / 99 \\
02 / 10 / 99 \\
02 / 10 / 99 \\
02 / 10 / 99 \\
02 / 10 / 99 \\
02 / 10 / 99 \\
02 / 10 / 99 \\
02 / 10 / 99 \\
02 / 10 / 99 \\
02 / 10 / 99 \\
02 / 10 / 99 \\
02 / 10 / 99 \\
02 / 10 / 99 \\
02 / 10 / 99 \\
02 / 10 / 99 \\
02 / 10 / 99 \\
02 / 10 / 99 \\
02 / 10 / 99 \\
02 / 10 / 99 \\
02 / 10 / 99 \\
02 / 10 / 99 \\
02 / 10 / 99 \\
02 / 10 / 99 \\
02 / 10 / 99 \\
02 / 10 / 99 \\
02 / 10 / 99 \\
02 / 10 / 99 \\
02 / 10 / 99 \\
02 / 10 / 99 \\
02 / 10 / 99 \\
02 / 10 / 99 \\
02 / 10 / 99 \\
02 / 10 / 99 \\
02 / 10 / 99 \\
02 / 10 / 99 \\
02 / 10 / 99 \\
02 / 10 / 99 \\
02 / 10 / 99 \\
02 / 10 / 99 \\
02 / 10 / 99 \\
02 / 10 / 99 \\
12 / 17 / 98 \\
12 / 17 / 98 \\
12 / 17 / 98 \\
12 / 17 / 98 \\
12 / 17 / 98 \\
12 / 17 / 98 \\
12 / 17 / 98 \\
12 / 17 / 98 \\
12 / 117 / 98 \\
12 / 17 / 98 \\
12 / 17 / 98 \\
12 / 17 / 98 \\
12 / 17 / 98\end{array}$ & 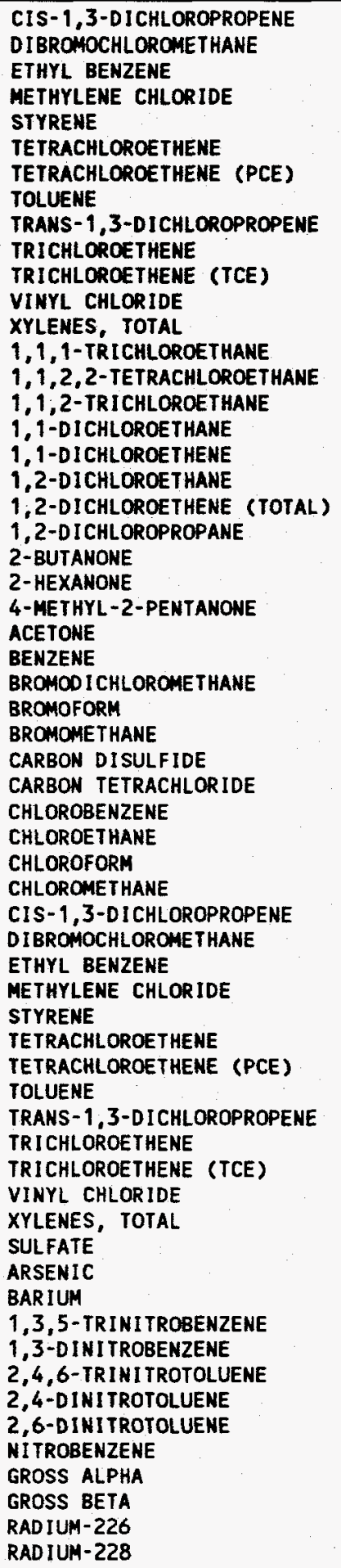 & 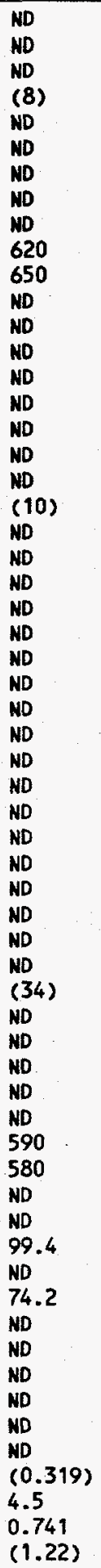 & $\begin{array}{l}50 \\
50 \\
50 \\
50 \\
50 \\
50 \\
25 \\
50 \\
50 \\
50 \\
25 \\
50 \\
50 \\
50 \\
50 \\
50 \\
50 \\
50 \\
50 \\
50 \\
50 \\
50 \\
50 \\
50 \\
50 \\
50 \\
50 \\
50 \\
50 \\
50 \\
50 \\
50 \\
50 \\
50 \\
50 \\
50 \\
50 \\
50 \\
50 \\
50 \\
50 \\
25 \\
50 \\
50 \\
50 \\
25 \\
50 \\
50 \\
0.076 \\
2.4 \\
0.1 \\
0.030 \\
0.090 \\
0.030 \\
0.030 \\
0.010 \\
0.030 \\
0.557 \\
0.737 \\
0.309 \\
1.28 \\
\end{array}$ & $\begin{array}{l}U G / L \\
U G / L \\
U G / L \\
U G / L \\
U G / L \\
U G / L \\
U G / L \\
U G / L \\
U G / L \\
U G / L \\
U G / L \\
U G / L \\
U G / L \\
U G / L \\
U G / L \\
U G / L \\
U G / L \\
U G / L \\
U G / L \\
U G / L \\
U G / L \\
U G / L \\
U G / L \\
U G / L \\
U G / L \\
U G / L \\
U G / L \\
U G / L \\
U G / L \\
U G / L \\
U G / L \\
U G / L \\
U G / L \\
U G / L \\
U G / L \\
U G / L \\
U G / L \\
U G / L \\
U G / L \\
U G / L \\
U G / L \\
U G / L \\
U G / L \\
U G / L \\
U G / L \\
U G / L \\
U G / L \\
U G / L \\
M G / L \\
U G / L \\
U G / L \\
U G / L \\
U G / L \\
U G / L \\
U G / L \\
U G / L \\
U G / L \\
P C I / L \\
P C I / L \\
P C I / L \\
\text { UCI/L }\end{array}$ & $\begin{array}{l}\text { EPA CLP } \\
\text { EPA CLP } \\
\text { EPA CLP } \\
\text { EPA CLP } \\
\text { EPA CLP } \\
\text { EPA CLP } \\
\text { EPA } 8260 \\
\text { EPA CLP } \\
\text { EPA CLP } \\
\text { EPA CLP } \\
\text { EPA } 8260 \\
\text { EPA CLP } \\
\text { EPA CLP } \\
\text { EPA CLP } \\
\text { EPA CLP } \\
\text { EPA CLP } \\
\text { EPA CLP } \\
\text { EPA CLP } \\
\text { EPA CLP } \\
\text { EPA CLP } \\
\text { EPA CLP } \\
\text { EPA CLP } \\
\text { EPA CLP } \\
\text { EPA CLP } \\
\text { EPA CLP } \\
\text { EPA CLP } \\
\text { EPA CLP } \\
\text { EPA CLP } \\
\text { EPA CLP } \\
\text { EPA CLP } \\
\text { EPA CLP } \\
\text { EPA CLP } \\
\text { EPA CLP } \\
\text { EPA CLP } \\
\text { EPA CLP } \\
\text { EPA CLP } \\
\text { EPA CLP } \\
\text { EPA CLP } \\
\text { EPA CLP } \\
\text { EPA CLP } \\
\text { EPA CLP } \\
\text { EPA } 8260 \\
\text { EPA CLP } \\
\text { EPA CLP } \\
\text { EPA CLP } \\
\text { EPA } 8260 \\
\text { EPA CLP } \\
\text { EPA CLP } \\
\text { EPA } 300.0 \\
\text { EPA CLP } \\
\text { EPA CLP } \\
\text { USATHAMA } \\
\text { USATHAMA } \\
\text { USATHAMA } \\
\text { USATHAMA } \\
\text { USATHAMA } \\
\text { USATHAMA } \\
\text { EPA } 900.0 \\
\text { EPA } 900.0 \\
\text { EPA } 903.1 \\
\text { EPA } 904.0\end{array}$ & . \\
\hline
\end{tabular}




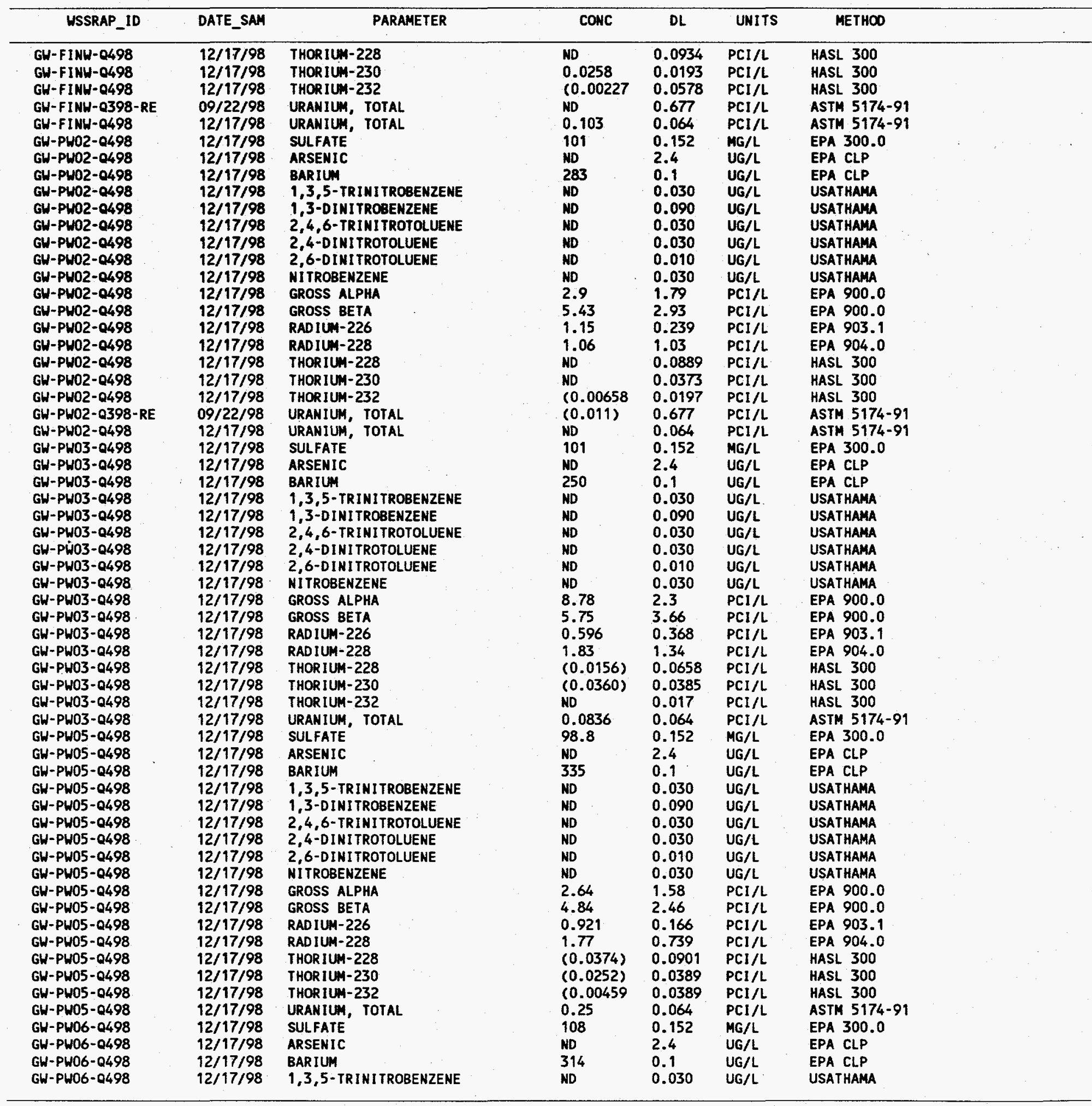




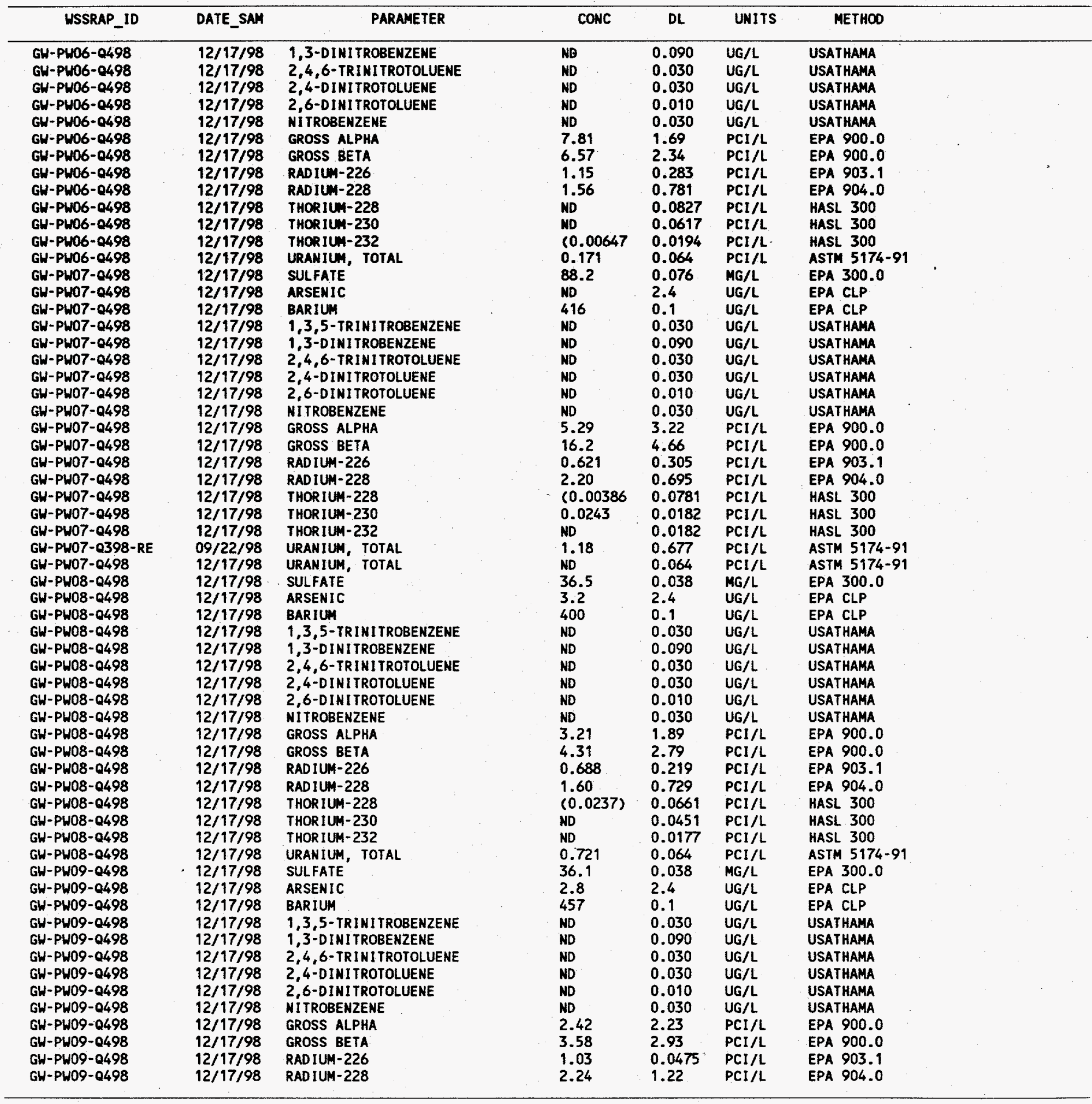




\begin{tabular}{|c|c|c|c|c|c|c|}
\hline WSSRAP_ID & DATE_SAM & PARAMETER & CONC & $\mathrm{DL}$ & UNITS & METHOD \\
\hline 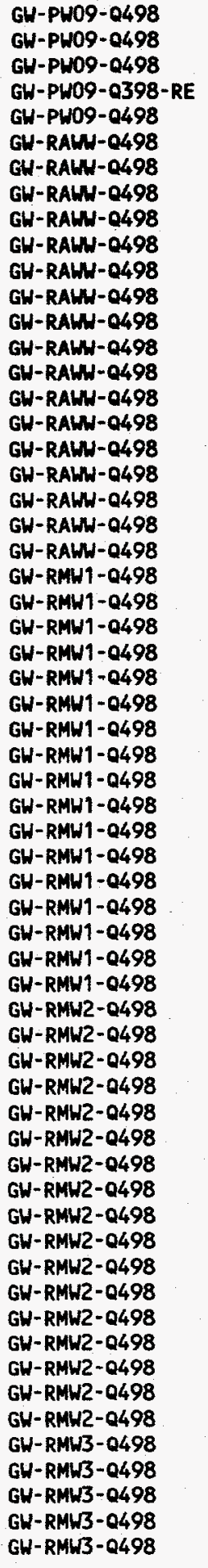 & $\begin{array}{l}12 / 17 / 98 \\
12 / 17 / 98 \\
12 / 17 / 98 \\
09 / 22 / 98 \\
12 / 17 / 98 \\
12 / 17 / 98 \\
12 / 17 / 98 \\
12 / 17 / 98 \\
12 / 17 / 98 \\
12 / 17 / 98 \\
12 / 17 / 98 \\
12 / 17 / 98 \\
12 / 17 / 98 \\
12 / 17 / 98 \\
12 / 17 / 98 \\
12 / 17 / 98 \\
12 / 17 / 98 \\
12 / 17 / 98 \\
12 / 17 / 98 \\
12 / 17 / 98 \\
12 / 17 / 98 \\
12 / 17 / 98 \\
12 / 16 / 98 \\
12 / 16 / 98 \\
12 / 16 / 98 \\
12 / 16 / 98 \\
12 / 16 / 98 \\
12 / 16 / 98 \\
12 / 16 / 98 \\
12 / 16 / 98 \\
12 / 16 / 98 \\
12 / 16 / 98 \\
12 / 16 / 98 \\
12 / 16 / 98 \\
12 / 16 / 98 \\
12 / 16 / 98 \\
12 / 16 / 98 \\
12 / 16 / 98 \\
12 / 16 / 98 \\
12 / 17 / 98 \\
12 / 17 / 98 \\
12 / 17 / 98 \\
12 / 17 / 98 \\
12 / 17 / 98 \\
12 / 17 / 98 \\
12 / 17 / 98 \\
12 / 17 / 98 \\
12 / 17 / 98 \\
12 / 17 / 98 \\
12 / 17 / 98 \\
12 / 17 / 98 \\
12 / 17 / 98 \\
12 / 17 / 98 \\
12 / 17 / 98 \\
12 / 17 / 98 \\
12 / 17 / 98 \\
12 / 16 / 98 \\
12 / 16 / 98 \\
12 / 16 / 98 \\
12 / 16 / 98 \\
12 / 16 / 98\end{array}$ & 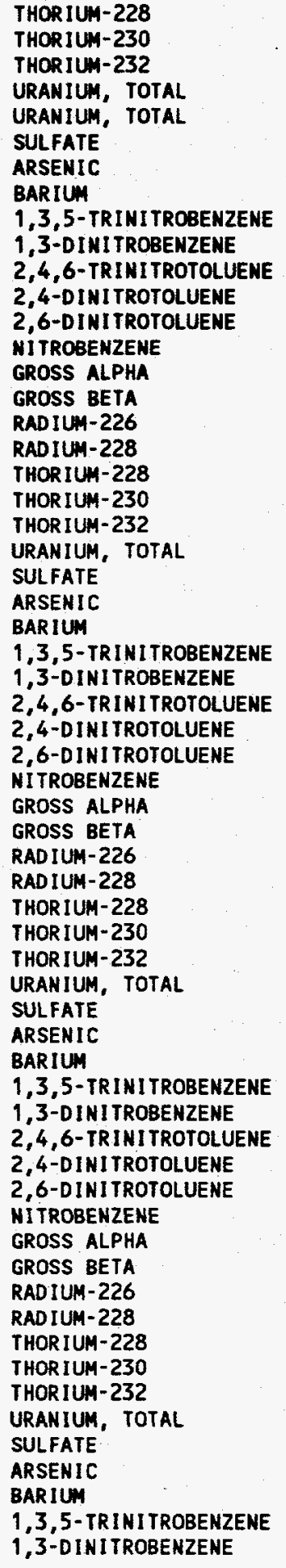 & 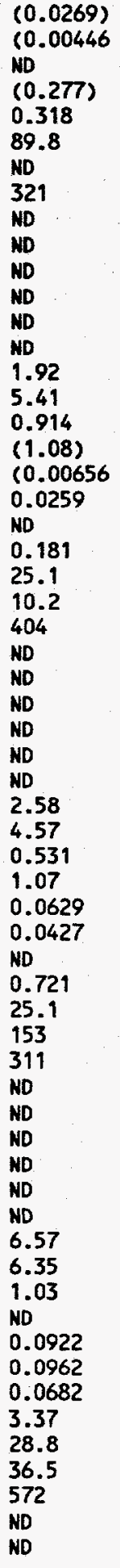 & $\begin{array}{l}0.0839 \\
0.0383 \\
0.0458 \\
0.677 \\
0.064 \\
0.152 \\
2.4 \\
0.1 \\
0.030 \\
0.090 \\
0.030 \\
0.030 \\
0.010 \\
0.030 \\
1 \\
1.71 \\
2.66 \\
0.305 \\
1 . .32 \\
0.019 \\
0.019 \\
0.03 \\
0\end{array}$ & $\begin{array}{l}P C I / L \\
P C I / L \\
P C I / L \\
P C I / L \\
P C I / L \\
M G / L \\
U G / L \\
U G / L \\
U G / L \\
U G / L \\
U G / L \\
U G / L \\
U G / L \\
U G / L \\
P C I / L \\
P C I / L \\
P C I / L \\
P C I / L \\
P C I / L \\
P C I / L \\
P C I / L \\
P C I / L \\
M G / L \\
U G / L \\
U G / L \\
U G / L \\
U G / L \\
U G / L \\
U G / L \\
U G / L \\
U G / L \\
P C I / L \\
P C I / L \\
P C I / L \\
P C I / L \\
P C I / L \\
P C I / L \\
P C I / L \\
P C I / L \\
M G / L \\
U G / L \\
U G / L \\
U G / L \\
U G / L \\
U G / L \\
U G / L \\
U G / L \\
U G / L \\
P C I / L \\
P C I / L \\
P C I / L \\
P C I / L \\
P C I / L \\
P C I / L \\
P C I / L \\
P C I / L \\
M G / L \\
U G / L \\
U G / L \\
U G / L \\
U G / L \\
\end{array}$ & $\begin{array}{l}\text { HASL } 300 \\
\text { HASL } 300 \\
\text { HASL } 300 \\
\text { ASTM } 5174-91 \\
\text { ASTM } 5174-91 \\
\text { EPA } 300.0 \\
\text { EPA CLP } \\
\text { EPA CLP } \\
\text { USATHAMA } \\
\text { USATHAMA } \\
\text { USATHAMA } \\
\text { USATHAMA } \\
\text { USATHAMA } \\
\text { USATHAMA } \\
\text { EPA } 900.0 \\
\text { EPA } 900.0 \\
\text { EPA } 903.1 \\
\text { EPA } 904.0 \\
\text { HASL } 300 \\
\text { HASL } 300 \\
\text { HASL } 300 \\
\text { ASTM } 5174-91 \\
\text { EPA } 300.0 \\
\text { EPA CLP } \\
\text { EPA CLP } \\
\text { USATHAMA } \\
\text { USATHAMA } \\
\text { USATHAMA } \\
\text { USATHAMA } \\
\text { USATHAMA } \\
\text { USATHAMA } \\
\text { EPA } 900.0 \\
\text { EPA } 900.0 \\
\text { EPA } 903.1 \\
\text { EPA } 904.0 \\
\text { HASL } 300 \\
\text { HASL } 300 \\
\text { HASL } 300 \\
\text { ASTM } 5174-91 \\
\text { EPA } 300.0 \\
\text { EPA CLP } \\
\text { EPA CLP } \\
\text { USATHAMA } \\
\text { USATHAMA } \\
\text { USATHAMA } \\
\text { USATHAMA } \\
\text { USATHAMA } \\
\text { USATHAMA } \\
\text { EPA } 900.0 \\
\text { EPA } 900.0 \\
\text { EPA } 903.1 \\
\text { EPA } 904.0 \\
\text { HASL } 300 \\
\text { HASL } 300 \\
\text { HASL } 300 \\
\text { ASTM } 5174-91 \\
\text { EPA } 300.0 \\
\text { EPA CLP } \\
\text { EPA CLP } \\
\text { USATHAMA } \\
\text { USATHAMA } \\
\text { USA }\end{array}$ \\
\hline
\end{tabular}




\begin{tabular}{|c|c|c|c|c|c|c|}
\hline WSSRAP_ID & DATE_SAM & PARAMETER & CONC & DL & UNITS & METHOD \\
\hline 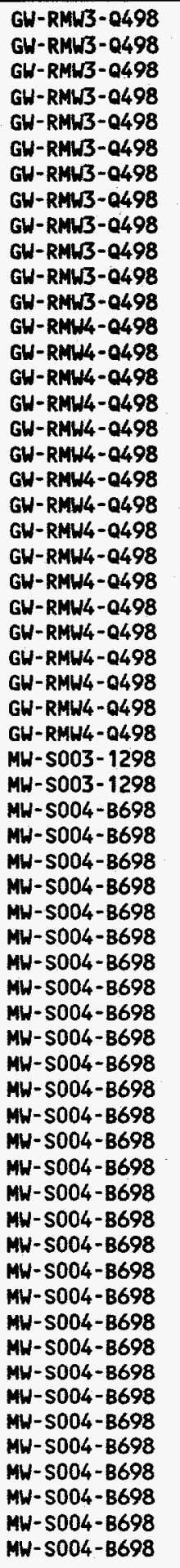 & $\begin{array}{l}12 / 16 / 98 \\
12 / 16 / 98 \\
12 / 16 / 98 \\
12 / 16 / 98 \\
12 / 16 / 98 \\
12 / 16 / 98 \\
12 / 16 / 98 \\
12 / 16 / 98 \\
12 / 16 / 98 \\
12 / 16 / 98 \\
12 / 16 / 98 \\
12 / 16 / 98 \\
12 / 16 / 98 \\
12 / 16 / 98 \\
12 / 16 / 98 \\
12 / 16 / 98 \\
12 / 16 / 98 \\
12 / 16 / 98 \\
12 / 16 / 98 \\
12 / 16 / 98 \\
12 / 16 / 98 \\
12 / 16 / 98 \\
12 / 16 / 98 \\
12 / 16 / 98 \\
12 / 16 / 98 \\
12 / 16 / 98 \\
12 / 16 / 98 \\
12 / 16 / 98 \\
12 / 16 / 98 \\
12 / 16 / 98 \\
12 / 16 / 98 \\
12 / 16 / 98 \\
12 / 16 / 98 \\
12 / 16 / 98 \\
12 / 16 / 98 \\
12 / 16 / 98 \\
12 / 16 / 98 \\
12 / 16 / 98 \\
12 / 16 / 98 \\
12 / 16 / 98 \\
12 / 16 / 98 \\
12 / 16 / 98 \\
12 / 16 / 98 \\
12 / 16 / 98 \\
12 / 16 / 98 \\
12 / 16 / 98 \\
12 / 16 / 98 \\
12 / 16 / 98 \\
12 / 16 / 98 \\
12 / 16 / 98 \\
12 / 16 / 98 \\
12 / 16 / 98 \\
12 / 16 / 98 \\
12 / 16 / 98 \\
12 / 16 / 98 \\
12 / 16 / 98 \\
12 / 16 / 98 \\
12 / 16 / 98 \\
12 / 16 / 98 \\
12 / 16 / 98 \\
12 / 16 / 98\end{array}$ & 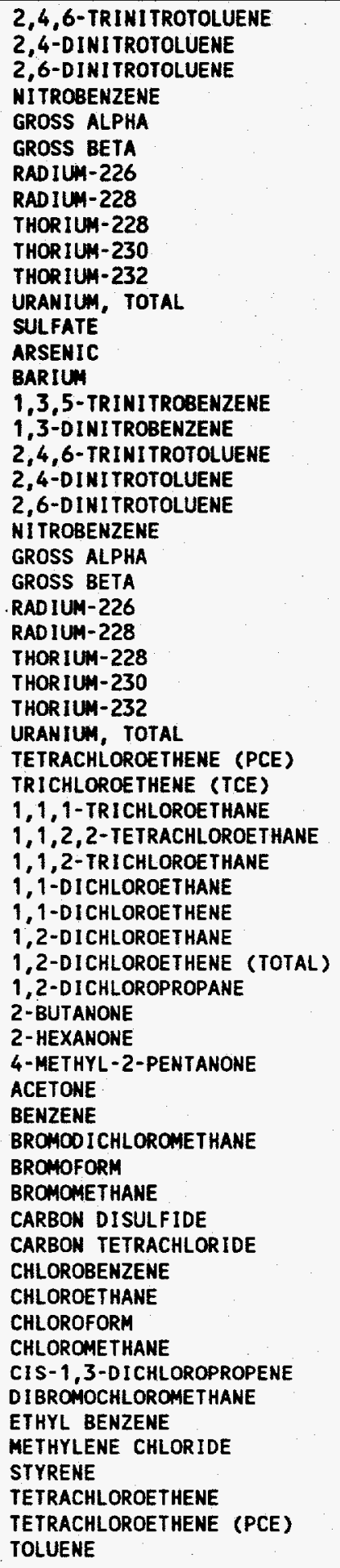 & 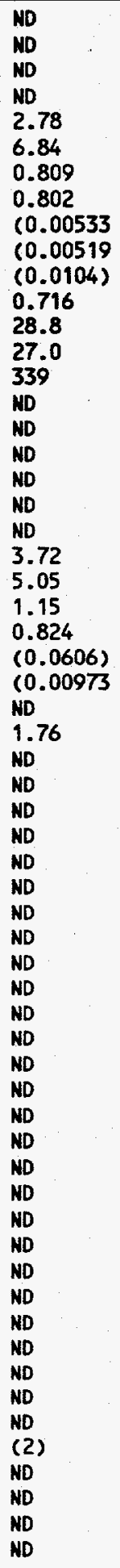 & 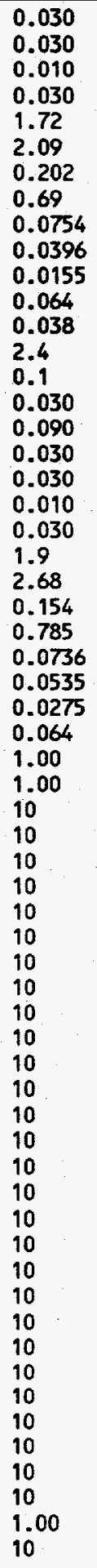 & $\begin{array}{l}U G / L \\
U G / L \\
U G / L \\
U G / L \\
P C I / L \\
P C I / L \\
P C I / L \\
P C I / L \\
P C I / L \\
P C I / L \\
P C I / L \\
P C I / L \\
U G / L \\
U G / L \\
U G / L \\
U G / L \\
U G / L \\
U G / L \\
U G / L \\
U G / L \\
U G / L \\
P C I / L \\
P C I / L \\
P C I / L \\
P C I / L \\
P C I / L \\
P C I / L \\
P C I / L \\
P C I / L \\
U G / L \\
U G / L \\
U G / L \\
U G / L \\
U G / L \\
U G / L \\
U G / L \\
U G / L \\
U G / L \\
U G / L \\
U G / L \\
U G / L \\
U G / L \\
U G / L \\
U G / L \\
U G / L \\
U G / L \\
U G / L \\
U G / L \\
U G / L \\
U G / L \\
U G / L \\
U G / L \\
U G / L \\
U G / L \\
U G / L \\
U G / L \\
U G / L \\
U G / L \\
U G / L \\
U G / L \\
U G / L \\
\end{array}$ & $\begin{array}{l}\text { USATHAMA } \\
\text { USATHAMA } \\
\text { USATHAMA } \\
\text { USATHAMA } \\
\text { EPA } 900.0 \\
\text { EPA } 900.0 \\
\text { EPA } 903.1 \\
\text { EPA } 904.0 \\
\text { HASL } 300 \\
\text { HASL } 300 \\
\text { HASL } 300 \\
\text { ASTM } 5174-91 \\
\text { EPA } 300.0 \\
\text { EPA CLP } \\
\text { EPA CLP } \\
\text { USATHAMA } \\
\text { USATHAMA } \\
\text { USATHAMA } \\
\text { USATHAMA } \\
\text { USATHAMA } \\
\text { USATHAMA } \\
\text { EPA } 900.0 \\
\text { EPA 900.0 } \\
\text { EPA } 903.1 \\
\text { EPA } 904.0 \\
\text { HASL } 300 \\
\text { HASL } 300 \\
\text { HASL } 300 \\
\text { ASTM } 5174-91 \\
\text { EPA } 8021 A \\
\text { EPA } 8021 A \\
\text { EPA CLP } \\
\text { EPA CLP } \\
\text { EPA CLP } \\
\text { EPA CLP } \\
\text { EPA CLP } \\
\text { EPA CLP } \\
\text { EPA CLP } \\
\text { EPA CLP } \\
\text { EPA CLP } \\
\text { EPA CLP } \\
\text { EPA CLP } \\
\text { EPA CLP } \\
\text { EPA CLP } \\
\text { EPA CLP } \\
\text { EPA CLP } \\
\text { EPA CLP } \\
\text { EPA CLP } \\
\text { EPA CLP } \\
\text { EPA CLP } \\
\text { EPA CLP } \\
\text { EPA CLP } \\
\text { EPA CLP } \\
\text { EPA CLP } \\
\text { EPA CLP } \\
\text { EPA CLP } \\
\text { EPA CLP } \\
\text { EPA CLP } \\
\text { EPA CLP } \\
\text { EPA } 8021 A \\
\text { EPA CLP } \\
\text { EPA }\end{array}$ \\
\hline
\end{tabular}




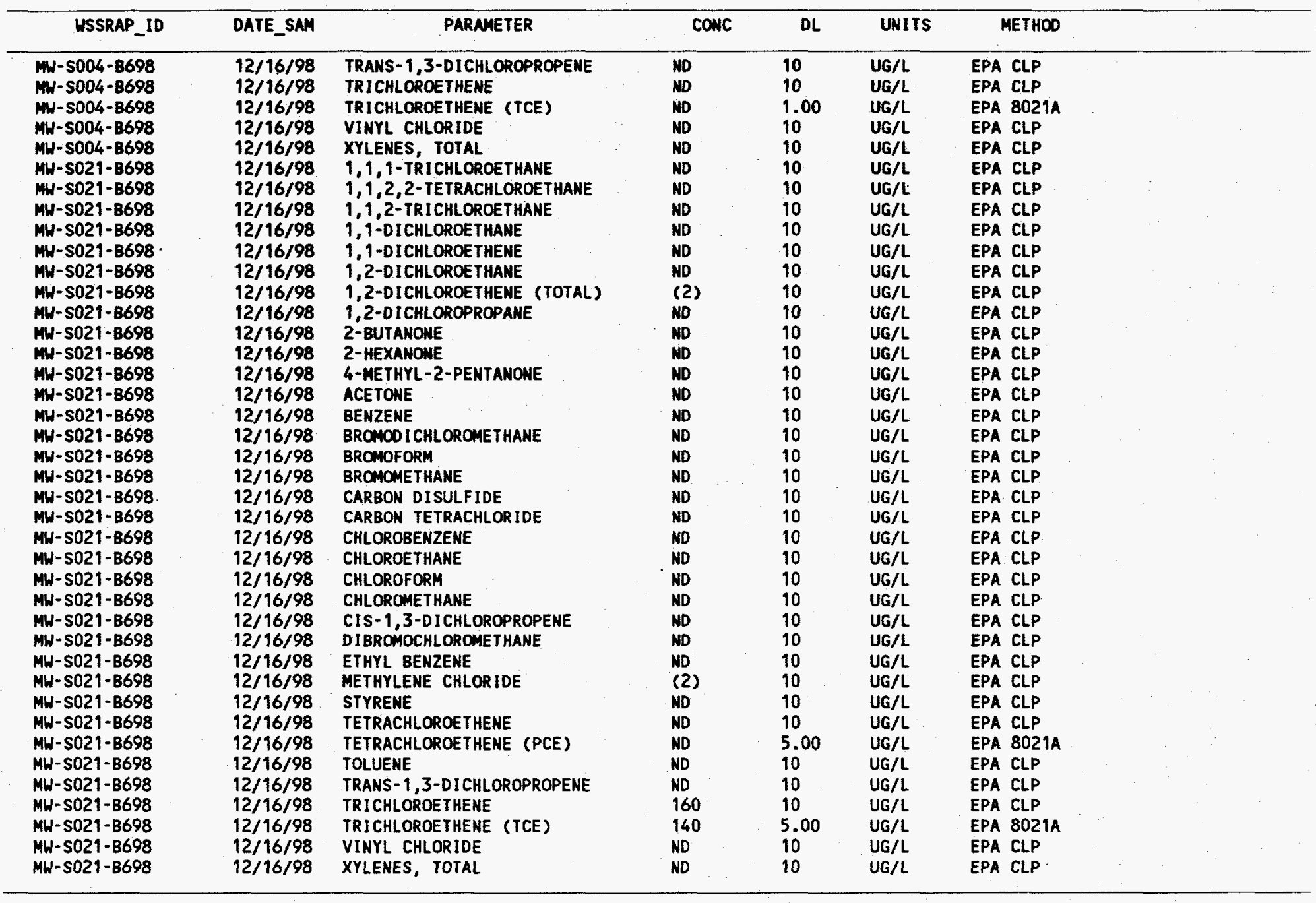


TOTAL URANIUM ON-SITE KPA ANALYSES, 1ST QUARTER, 1999

\begin{tabular}{|c|c|c|c|c|c|c|c|}
\hline SAMPLE ID & LOC. & DATE & PARAMETER & CONCENTRATION & ERROR & D.L. & UNITS \\
\hline GW-1002-Q199 & 1002 & $1 / 27 / 99$ & URANIUM,TOTAL & 4.43 & 0.07 & 0.7 & $\mathrm{PCl} / \mathrm{L}$ \\
\hline GW-1004-Q199 & 1004 & $1 / 27 / 99$ & URANIUM,TOTAL & 1870 & 65.18 & 0.7 & $\mathrm{PCI} / \mathrm{L}$ \\
\hline GW-1005-Q199 & 1005 & $1 / 27 / 99$ & URANIUM,TOTAL & 1250 & 43.9 & 0.7 & $\mathrm{PCl} / \mathrm{L}$ \\
\hline GW-1006-Q199 & 1006 & $2 / 1 / 99$ & URANIUM,TOTAL & 1910 & 67.4 & 0.7 & $\mathrm{PCI} / \mathrm{L}$ \\
\hline GW-1007-Q199 & 1007 & $2 / 1 / 99$ & URANIUM,TOTAL & 5.92 & 0.15 & 0.7 & $\mathrm{PCI} / \mathrm{L}$ \\
\hline GW-1008-Q199 & 1008 & $2 / 1 / 99$ & URANIUM,TOTAL & 679.84 & 23.8 & 0.7 & $P C I / L$ \\
\hline GW-1009-Q199 & 1009 & $2 / 1 / 99$ & URANIUM,TOTAL & 0.51 & 0.03 & 0.7 & $\mathrm{PCI} / \mathrm{L}$ \\
\hline GW-1013-Q199 & 1013 & $2 / 1 / 99$ & URANIUM,TOTAL & 599.52 & 21.36 & 0.7 & $\mathrm{PCI} / \mathrm{L}$ \\
\hline GW-1014-Q199 & 1014 & $2 / 1 / 99$ & URANIUM,TOTAL & 860.1 & 30.18 & 0.7 & $\overline{P C I / L}$ \\
\hline GW-1015-Q199 & 1015 & $2 / 1 / 99$ & URANIUM,TOTAL & 183.8 & 6.47 & 0.7 & $\mathrm{PCI} / \mathrm{L}$ \\
\hline GW-1016-Q199 & 1016 & $2 / 1 / 99$ & URANIUM,TOTAL & 118.84 & 4.17 & 0.7 & $\mathrm{PCI} / \mathrm{L}$ \\
\hline GW-1017-Q199 & 1017 & $3 / 12 / 99$ & URANIUM,TOTAL & 10.17 & 2.53 & 0.7 & $\overline{P C I / L}$ \\
\hline GW-1018-Q199 & 1018 & $3 / 12 / 99$ & URANIUM,TOTAL & ND & & 1.4 & $\mathrm{PCI} / \mathrm{L}$ \\
\hline GW-1019-Q199 & 1019 & $3 / 12 / 99$ & URANIUM,TOTAL & 1.36 & 0.48 & 0.7 & $\mathrm{PCI} / \mathrm{L}$ \\
\hline GW-1020-Q199 & 1020 & $3 / 12 / 99$ & URANIUM,TOTAL & 0.82 & 0.12 & 0.7 & $\mathrm{PCI} / \mathrm{L}$ \\
\hline GW-1021-Q199 & 1021 & $3 / 8 / 99$ & URANIUM,TOTAL & ND & & 1.4 & $\mathrm{PCI} / \mathrm{L}$ \\
\hline GW-1022-Q199 & 1022 & $3 / 8 / 99$ & URANIUM,TOTAL & ND & & 1.4 & $\mathrm{PCI} / \mathrm{L}$ \\
\hline GW-1023-Q199 & 1023 & $3 / 12 / 99$ & URANIUM,TOTAL & ND & & 1.4 & $\mathrm{PCI} / \mathrm{L}$ \\
\hline GW-1024-Q199 & 1024 & $3 / 16 / 99$ & URANIUM,TOTAL & 2.97 & 0.55 & 0.7 & $\mathrm{PCl} / \mathrm{L}$ \\
\hline GW-1026-Q199 & 1026 & $1 / 12 / 99$ & URANIUM,TOTAL & 0.43 & 0.01 & 0.7 & $\mathrm{PCI} / \mathrm{L}$ \\
\hline GW-1027-Q199 & 1027 & $1 / 15 / 99$ & URANIUM,TOTAL & 194.64 & 6.91 & 0.7 & $\overline{P C I / L}$ \\
\hline GW-1029-Q199 & 1029 & $1 / 27 / 99$ & URANIUM,TOTAL & 2.57 & 0.05 & 0.7 & $\mathrm{PCI} / \mathrm{L}$ \\
\hline GW-1030-Q199 & 1030 & $1 / 27 / 99$ & URANIUM,TOTAL & 15.09 & 0.67 & 0.7 & $\mathrm{PCI} / \mathrm{L}$ \\
\hline GW-1031-Q199 & 1031 & $2 / 1 / 99$ & URANIUM,TOTAL & 91.6 & 3.28 & 0.7 & PCI/L \\
\hline GW-1032-Q199 & 1032 & $2 / 1 / 99$ & URANIUM,TOTAL & 1020 & 35.95 & 0.7 & $\mathrm{PCI} / \mathrm{L}$ \\
\hline GW-1033-Q199 & 1033 & $3 / 8 / 99$ & URANIUM,TOTAL & 1.1 & 0.04 & 0.7 & $\mathrm{PCI} / \mathrm{L}$ \\
\hline GW-1034-Q199 & 1034 & $1 / 12 / 99$ & URANIUM,TOTAL & 3.03 & 0.04 & 0.7 & $\mathrm{PCl} / \mathrm{L}$ \\
\hline GW-1035-Q199 & 1035 & $1 / 15 / 99$ & URANIUM,TOTAL & 0.03 & 0.01 & 0.7 & $\mathrm{PCI} / \mathrm{L}$ \\
\hline GW-1036-Q199 & 1036 & $1 / 19 / 99$ & URANIUM,TOTAL & 5.94 & 0.1 & 0.7 & $\mathrm{PCI} / \mathrm{L}$ \\
\hline GW-1037-Q199 & 1037 & $1 / 19 / 99$ & URANIUM,TOTAL & 0.47 & 0.01 & 0.7 & $P C I / L$ \\
\hline GW-1040-Q199 & 1040 & $1 / 15 / 99$ & URANIUM,TOTAL & 9.23 & 0.16 & 0.7 & PCI/L \\
\hline GW-1041-Q199 & 1041 & $1 / 15 / 99$ & URANIUM,TOTAL & 6.51 & 0.26 & 0.7 & $P C \mid / L$ \\
\hline GW-1042-Q199 & 1042 & $1 / 12 / 99$ & URANIUM,TOTAL & 1.27 & 0.02 & 0.7 & $\mathrm{PCI} / \mathrm{L}$ \\
\hline GW-1043-Q199 & 1043 & $1 / 12 / 99$ & URANIUM,TOTAL & 1.16 & 0.02 & 0.7 & $\mathrm{PCI} / \mathrm{L}$ \\
\hline GW-1044-Q199 & 1044 & $3 / 12 / 99$ & URANIUM,TOTAL & ND & & 1.4 & $\mathrm{PCI} / \mathrm{L}$ \\
\hline GW-1046-Q199 & 1046 & $2 / 2 / 99$ & URANIUM,TOTAL & 3.79 & 0.05 & 0.7 & $P C I / L$ \\
\hline GW-1047-Q199 & 1047 & $2 / 2 / 99$ & URANIUM,TOTAL & 0.83 & 0.04 & 0.7 & $\mathrm{PCl} / \mathrm{L}$ \\
\hline GW-1048-Q199 & 1048 & $2 / 2 / 99$ & URANIUM,TOTAL & 408.39 & 14.31 & 0.7 & $\mathrm{PCl} / \mathrm{L}$ \\
\hline GW-1049-Q199 & 1049 & $2 / 2 / 99$ & URANIUM,TOTAL & 7.36 & 0.18 & 0.7 & $\mathrm{PCl} / \mathrm{L}$ \\
\hline GW-2035-Q199 & 2035 & $2 / 9 / 99$ & URANIUM, TOTAL & 0.16 & 0.01 & 0.7 & $\mathrm{PCI} / \mathrm{L}$ \\
\hline GW-2036-Q199 & 2036 & $2 / 9 / 99$ & URANIUM,TOTAL & 1.3 & 0.02 & 0.7 & $\mathrm{PCI} / \mathrm{L}$ \\
\hline GW-2037-B199 & 2037 & $1 / 20 / 99$ & URANIUM,TOTAL & 1.47 & 0.26 & 0.7 & $\mathrm{PCI} / \mathrm{L}$ \\
\hline GW-2037-B299 & 2037 & $3 / 17 / 99$ & URANIUM,TOTAL & ND & & 0.7 & $\mathrm{PCl} / \mathrm{L}$ \\
\hline GW-2038-B199 & 2038 & $1 / 20 / 99$ & URANIUM,TOTAL & 2.21 & 0.16 & 0.7 & $\mathrm{PCl} / \mathrm{L}$ \\
\hline GW-2038-B299 & 2038 & $3 / 17 / 99$ & URANIUM,TOTAL & 2.59 & 0.09 & 0.7 & $\mathrm{PCI} / \mathrm{L}$ \\
\hline GW-2039-B199 & 2039 & $1 / 20 / 99$ & URANIUM,TOTAL & 3.04 & 0.04 & 0.7 & $\mathrm{PCl} / \mathrm{L}$ \\
\hline GW-2039-B299 & 2039 & $3 / 17 / 99$ & URANIUM,TOTAL & 3.13 & 0.1 & 0.7 & $\mathrm{PCI} / \mathrm{L}$ \\
\hline GW-2040-Q199 & 2040 & $2 / 4 / 99$ & URANIUM,TOTAL & 1.7 & 0.02 & 0.7 & $\mathrm{PCI} / \mathrm{L}$ \\
\hline GW-2041-Q199 & 2041 & $2 / 4 / 99$ & URANIUM,TOTAL & 4.61 & 0.06 & 0.7 & $\mathrm{PCl} / \mathrm{L}$ \\
\hline GW-2042-Q199 & 2042 & $2 / 4 / 99$ & URANIUM,TOTAL & 2.32 & 0.03 & 0.7 & $\mathrm{PCI} / \mathrm{L}$ \\
\hline GW-2043-Q199 & 2043 & $2 / 4 / 99$ & URANIUM,TOTAL & 1.36 & 0.03 & 0.7 & $\mathrm{PCl} / \mathrm{L}$ \\
\hline
\end{tabular}


TOTAL URANIUM ON-SITE KPA ANALYSES, 1ST QUARTER, 1999

\begin{tabular}{|c|c|c|c|c|c|c|c|}
\hline SAMPLE ID & LOC. & DATE & PARAMETER & CONCENTRATION & ERROR & D.L. & UNITS \\
\hline GW-3003-B199 & 3003 & $2 / 3 / 99$ & URANIUM,TOTAL & 14.39 & 0.19 & 0.7 & $\mathrm{PCl} / \mathrm{L}$ \\
\hline GW-3003-B299 & 3003 & $4 / 8 / 99$ & URANIUM,TOTAL & 18.54 & 1.09 & 1.4 & PCI/L \\
\hline GW-3023-B199 & 3023 & $2 / 3 / 99$ & URANIUM,TOTAL & 8.1 & 0.12 & 0.7 & $\mathrm{PCI} / \mathrm{L}$ \\
\hline GW-3023-B299 & 3023 & $4 / 8 / 99$ & URANIUM,TOTAL & 9.92 & 0.21 & 1.4 & $\mathrm{PCI} / \mathrm{L}$ \\
\hline GW-3024-B199 & 3024 & $1 / 21 / 99$ & URANIUM,TOTAL & 55.78 & 2.13 & 0.7 & $\mathrm{PCl} / \mathrm{L}$ \\
\hline GW-3024-B299 & 3024 & $3 / 22 / 99$ & URANIUM,TOTAL & 63.47 & 3.69 & 1.4 & $\mathrm{PCl} / \mathrm{L}$ \\
\hline GW-3025-B199 & 3025 & $1 / 21 / 99$ & URANIUM,TOTAL & 2.31 & 0.03 & 0.7 & $\mathrm{PCI} / \mathrm{L}$ \\
\hline GW-3025-B299 & 3025 & $3 / 22 / 99$ & URANIUM,TOTAL & 3.01 & 0.1 & 1.4 & $\overline{\mathrm{PCl} / \mathrm{L}}$ \\
\hline GW-3027-B199 & 3027 & $1 / 19 / 99$ & URANIUM,TOTAL & 0.89 & 0.01 & 0.7 & $\mathrm{PCl} / \mathrm{L}$ \\
\hline GW-3027-B299 & 3027 & $3 / 22 / 99$ & URANIUM,TOTAL & 1.14 & 0.04 & 1.4 & $\mathrm{PCl} / \mathrm{L}$ \\
\hline GW-4001-B199 & 4001 & $2 / 8 / 99$ & URANIUM,TOTAL & 0.08 & 0.01 & 0.7 & $\overline{P C l} / \mathrm{L}$ \\
\hline GW-4001-B299 & 4001 & $4 / 7 / 99$ & URANIUM,TOTAL & 0.47 & 0.02 & 1.4 & $\mathrm{PCI} / \mathrm{L}$ \\
\hline GW-4002-B199 & 4002 & $2 / 3 / 99$ & URANIUM,TOTAL & 0.4 & 0.01 & 0.7 & $\mathrm{PCI} / \mathrm{L}$ \\
\hline GW-4002-B299 & 4002 & $4 / 8 / 99$ & URANIUM,TOTAL & 0.45 & 0.02 & 1.4 & $\mathrm{PCl} / \mathrm{L}$ \\
\hline GW-4006-B199 & 4006 & $2 / 3 / 99$ & URANIUM,TOTAL & 0.19 & 0.01 & 0.7 & $\mathrm{PCI} / \mathrm{L}$ \\
\hline GW-4006-B299 & 4006 & $4 / 7 / 99$ & URANIUM,TOTAL & 0.67 & 0.04 & 1.4 & $\mathrm{PCl} / \mathrm{L}$ \\
\hline GW-2048-B199 & 4028 & $2 / 10 / 99$ & URANIUM,TOTAL & 0.63 & 0.01 & 0.7 & $\mathrm{PCI} / \mathrm{L}$ \\
\hline GW-FINW-Q199 & FINW & $3 / 18 / 99$ & URANIUM,TOTAL & ND & & 1.4 & $\mathrm{PCl} / \mathrm{L}$ \\
\hline GW-PW02-Q199 & PW02 & $3 / 18 / 99$ & URANIUM,TOTAL & ND & & 7.0 & $\mathrm{PCI} / \mathrm{L}$ \\
\hline GW-PW03-Q199 & PW03 & $3 / 18 / 99$ & URANIUM,TOTAL & 1.31 & 0.36 & 1.4 & $\mathrm{PCI} / \mathrm{L}$ \\
\hline GW-PW05-Q199 & PW05 & $3 / 18 / 99$ & URANIUM,TOTAL & ND & & 1.4 & $\overline{P C l} / \mathrm{L}$ \\
\hline GW-PW06-Q199 & PW06 & $3 / 18 / 99$ & URANIUM,TOTAL & 0.81 & 0.16 & 1.4 & $\mathrm{PCI} / \mathrm{L}$ \\
\hline GW-PW07-Q199 & PW07 & $3 / 18 / 99$ & URANIUM,TOTAL & ND & & 7.0 & $\mathrm{PCI} / \mathrm{L}$ \\
\hline GW-PW08-Q199 & PW08 & $3 / 18 / 99$ & URANIUM,TOTAL & 0.63 & 0.06 & 1.4 & $\mathrm{PCl} / \mathrm{L}$ \\
\hline GW-PW09-Q199 & PW09 & $3 / 18 / 99$ & URANIUM,TOTAL & 0.53 & 0.09 & 1.4 & $\mathrm{PCI} / \mathrm{L}$ \\
\hline GW-RAWW-Q199 & RAWW & $3 / 18 / 99$ & URANIUM,TOTAL & ND & & 1.4 & $\mathrm{PCl} / \mathrm{L}$ \\
\hline GW-RMW1-Q199 & RMW1 & $3 / 16 / 99$ & URANIUM,TOTAL & 0.89 & 0.08 & 0.7 & $\mathrm{PCI} / \mathrm{L}$ \\
\hline GW-RMW2-Q199 & RMW2 & $3 / 18 / 99$ & URANIUM,TOTAL & 4.3 & 0.11 & 1.4 & $\mathrm{PCI} / \mathrm{L}$ \\
\hline GW-RMW3-Q199 & RMW3 & $3 / 16 / 99$ & URANIUM,TOTAL & 0.94 & 0.1 & 0.7 & $\mathrm{PCl} / \mathrm{L}$ \\
\hline GW-RMW4-Q199 & RMW4 & $3 / 16 / 99$ & URANIUM,TOTAL & 1.69 & 0.09 & 0.7 & $\mathrm{PCI} / \mathrm{L}$ \\
\hline SP-6301-Q199-L & 6301 & $2 / 15 / 99$ & URANIUM,TOTAL & 25.3 & 0.94 & 1 & $\mathrm{PCl} / \mathrm{L}$ \\
\hline SW-1003-Q199 & 1003 & $2 / 17 / 99$ & URANIUM,TOTAL & 40.09 & 1.44 & 1 & $\mathrm{PCl} / \mathrm{L}$ \\
\hline SW-1004-Q199 & 1004 & $2 / 17 / 99$ & URANIUM,TOTAL & 55.52 & 1.98 & 1 & $\mathrm{PCl} / \mathrm{L}$ \\
\hline SW-1005-Q199 & 1005 & $2 / 17 / 99$ & URANIUM,TOTAL & 28.17 & 1 & 1 & $\mathrm{PCl} / \mathrm{L}$ \\
\hline SW-1007-Q199 & 1007 & $2 / 17 / 99$ & URANIUM,TOTAL & 13.39 & 0.51 & $\overline{1}$ & $\mathrm{PCl} / \mathrm{L}$ \\
\hline SW-1009-Q199 & 1009 & $2 / 17 / 99$ & URANIUM,TOTAL & 10.69 & 0.13 & 1 & $\mathrm{PCI} / \mathrm{L}$ \\
\hline SW-1010-Q199 & 1010 & $2 / 17 / 99$ & URANIUM,TOTAL & 26.82 & 0.96 & 1 & $\overline{P C l} / \mathrm{L}$ \\
\hline SW-2004-Q199 & 2004 & $2 / 17 / 99$ & URANIUM,TOTAL & 6.33 & 0.08 & 1 & $\mathrm{PCI} / \mathrm{L}$ \\
\hline SW-2005-Q199 & 2005 & $2 / 17 / 99$ & URANIUM,TOTAL & 5.96 & 0.08 & 1 & $\mathrm{PCl} / \mathrm{L}$ \\
\hline SW-2012-Q199 & 2012 & $2 / 17 / 99$ & URANIUM,TOTAL & 3.7 & 0.05 & 1 & $\mathrm{PCl} / \mathrm{L}$ \\
\hline SW-2016-Q199 & 2016 & $2 / 17 / 99$ & URANIUM,TOTAL & 0.58 & 0.01 & 1 & $\overline{P C I / L}$ \\
\hline SW-2024-Q199 & 2024 & $2 / 17 / 99$ & URANIUM,TOTAL & 1.15 & 0.02 & 0.7 & $\overline{\mathrm{PCl} / \mathrm{L}}$ \\
\hline
\end{tabular}


SURFACE WATER 
SURFACE WATER

DATA MERGED DURING FIRST QUARTER, 1999

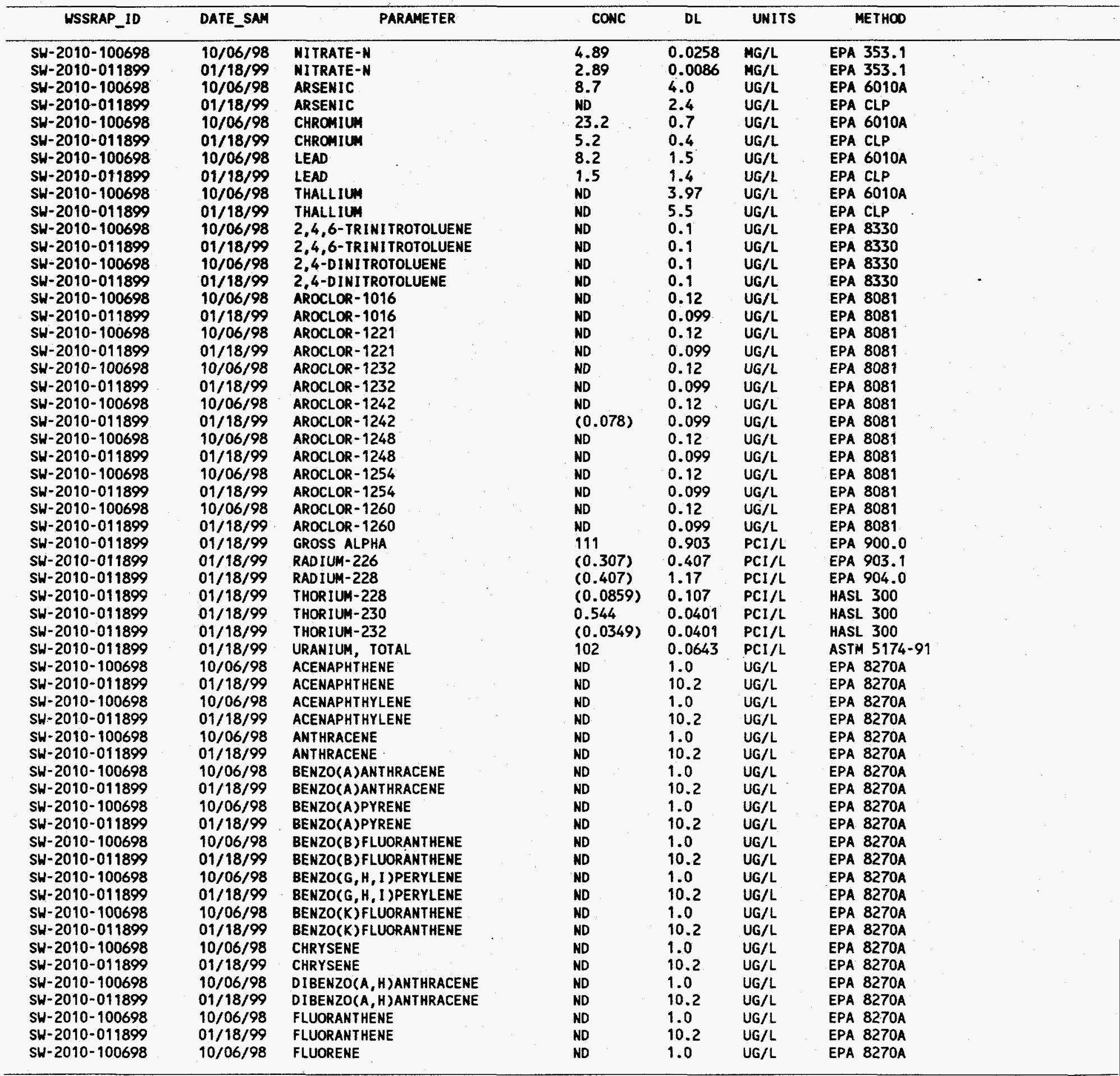




\begin{tabular}{|c|c|c|c|c|c|c|}
\hline USSRAP_ID & DATE_SAM & PARAMETER & CONC & $\mathrm{DL}$ & UNITS & METHOD \\
\hline $\begin{array}{l}\text { SW-2010-011899 } \\
\text { SW-2010-100698 } \\
\text { SW-2010-011899 } \\
\text { SW-2010-100698 } \\
\text { SW-2010-011899 } \\
\text { SW-2010-100698 } \\
\text { SW-2010-011899 } \\
\text { SW-2010-100698 } \\
\text { SW-2010-011899 } \\
\text { SW-2018-102698 } \\
\text { SW-2018-020899 } \\
\text { SW-2018-102698 } \\
\text { SW-2018-020899 } \\
\text { SW-2018-102698 } \\
\text { SW-2018-020899 } \\
\text { SW-2018-102698 } \\
\text { SW-2018-020899 } \\
\text { SW-2018-020899 } \\
\text { SW-2018-020899 } \\
\text { SW-2018-020899 } \\
\text { SW-2018-020899 } \\
\text { SW-2018-020899 } \\
\text { SW-2018-020899 } \\
\text { SW-2018-020899 } \\
\text { SW-2018-020899 } \\
\text { SW-2018-020899 } \\
\text { SW-2018-020899 } \\
\text { SW-2018-020899 } \\
\text { SW-2018-020899 } \\
\text { SW-2018-020899 } \\
\text { SW-2018-020899 } \\
\text { SW-2018-020899 } \\
\text { SW-2018-020899 }\end{array}$ & $\begin{array}{l}01 / 18 / 99 \\
10 / 06 / 98 \\
01 / 18 / 99 \\
10 / 06 / 98 \\
01 / 18 / 99 \\
10 / 06 / 98 \\
01 / 18 / 99 \\
10 / 06 / 98 \\
01 / 18 / 99 \\
10 / 26 / 98 \\
02 / 08 / 99 \\
10 / 26 / 98 \\
02 / 08 / 99 \\
10 / 26 / 98 \\
02 / 08 / 99 \\
10 / 26 / 98 \\
02 / 08 / 99 \\
02 / 08 / 99 \\
02 / 08 / 99 \\
02 / 08 / 99 \\
02 / 08 / 99 \\
02 / 08 / 99 \\
02 / 08 / 99 \\
02 / 08 / 99 \\
02 / 08 / 99 \\
02 / 08 / 99 \\
02 / 08 / 99 \\
02 / 08 / 99 \\
02 / 08 / 99 \\
02 / 08 / 99 \\
02 / 08 / 99 \\
02 / 08 / 99 \\
02 / 08 / 99\end{array}$ & $\begin{array}{l}\text { FLUORENE } \\
\text { INDENO(1,2,3-CD)PYRENE } \\
\text { INDENO } 1,2,3-\text { CD }) \text { PYRENE } \\
\text { NAPHTHALENE } \\
\text { NAPHTHALENE } \\
\text { PHENANTHRENE } \\
\text { PHENANTHRENE } \\
\text { PYRENE } \\
\text { PYRENE } \\
\text { NITRATE-N } \\
\text { NITRATE-N } \\
\text { COPPER } \\
\text { COPPER } \\
\text { ZINC } \\
\text { ZINC } \\
\text { CHEMICAL OXYGEN DEMAND } \\
\text { CHEMICAL OXYGEN DEMAND } \\
\text { ACENAPHTHENE } \\
\text { ACENAPHTHYLENE } \\
\text { ANTHRACENE } \\
\text { BENZO(A)ANTHRACENE } \\
\text { BENZO(A)PYRENE } \\
\text { BENZO(B)FLUORANTHENE } \\
\text { BENZO(G, H, I)PERYLENE } \\
\text { BENZO(K) FLUORANTHENE } \\
\text { CHRYSENE } \\
\text { DIBENZO(A,H)ANTHRACENE } \\
\text { FLUORANTHENE } \\
\text { FLUORENE } \\
\text { INDENO(1,2,3-CD)PYRENE } \\
\text { NAPHTHALENE } \\
\text { PHENANTHRENE } \\
\text { PYRENE }\end{array}$ & $\begin{array}{l}\text { ND } \\
\text { ND } \\
\text { ND } \\
\text { ND } \\
\text { ND } \\
\text { ND } \\
\text { ND } \\
\text { ND } \\
\text { ND } \\
0.050 \\
2.51 \\
\text { ND } \\
6.4 \\
18.3 \\
17.4 \\
77.9 \\
\text { S1.1 } \\
\text { ND } \\
\text { ND } \\
\text { ND } \\
\text { ND } \\
\text { ND } \\
\text { ND } \\
\text { ND } \\
\text { ND } \\
\text { ND } \\
\text { ND } \\
\text { ND } \\
\text { ND } \\
\text { ND } \\
\text { ND } \\
\text { ND } \\
\text { ND }\end{array}$ & 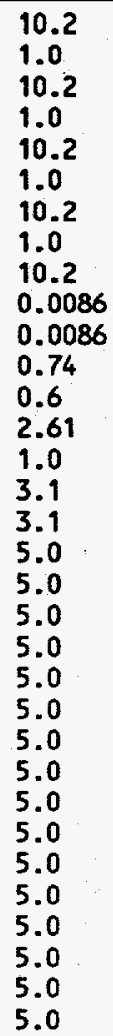 & $\begin{array}{l}U G / L \\
U G / L \\
U G / L \\
U G / L \\
U G / L \\
U G / L \\
U G / L \\
U G / L \\
U G / L \\
\text { UG/L } \\
\text { UG/L } \\
U G / L \\
U G / L \\
U G / L \\
U G / L \\
\text { UG/L } \\
\text { UG/L } \\
U G / L \\
U G / L \\
U G / L \\
U G / L \\
U G / L \\
U G / L \\
U G / L \\
U G / L \\
U G / L \\
U G / L \\
U G / L \\
U G / L \\
U G / L \\
U G / L \\
U G / L \\
U G / L\end{array}$ & 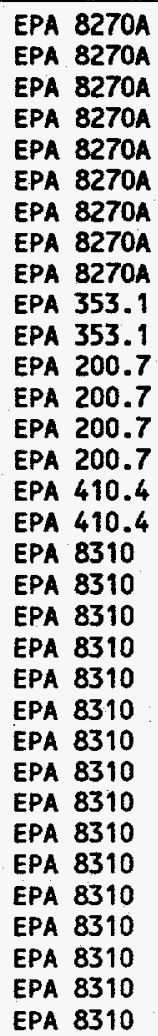 \\
\hline
\end{tabular}


DOWNSTREAM SURFACE WATER URANIUM RESULTS First Quarter 1999

\begin{tabular}{|c|c|c|c|c|}
\hline WSSRAP ID & DATE & PARAMETER & CONCENTRATION & UNITS \\
\hline SW-2004-Q199 & $2 / 17 / 99$ & Uranium, Total & 6.33 & $\mathrm{pCi} / 1$ \\
\hline SW-2005-Q199 & $2 / 17 / 99$ & Uranium, Total & 5.96 & $\mathrm{pCi} /$ \\
\hline SW-2012-Q199 & $2 / 17 / 99$ & Uranium, Total & 3.70 & $\mathrm{pCi} / 1$ \\
\hline SW-2024-Q199 & $2 / 17 / 99$ & Uranium, Total & 1.15 & $\mathrm{pCi} / 1$ \\
\hline SW-1003-Q199 & $2 / 17 / 99$ & Uranium, Total & 40.09 & $\mathrm{pCi} / 1$ \\
\hline SW-1004-Q199 & $2 / 17 / 99$ & Uranium, Total & 55.52 & $\mathrm{pCi} / 1$ \\
\hline SW-1005-Q199 & $2 / 17 / 99$ & Uranium, Total & 28.17 & $\mathrm{pCi} / 1$ \\
\hline SW-1007-Q199 & $2 / 17 / 99$ & Uranium, Total & 13.39 & $\mathrm{pCi} / 1$ \\
\hline SW-1009-Q199 & $2 / 17 / 99$ & Uranium, Total & 10.69 & $\mathrm{pCi} / 1$ \\
\hline SW-1010-Q19 & $2 / 17 / 00$ & Uranium, Total & 26.82 & $\mathrm{pCi} / 1$ \\
\hline
\end{tabular}




\section{SPRINGS}




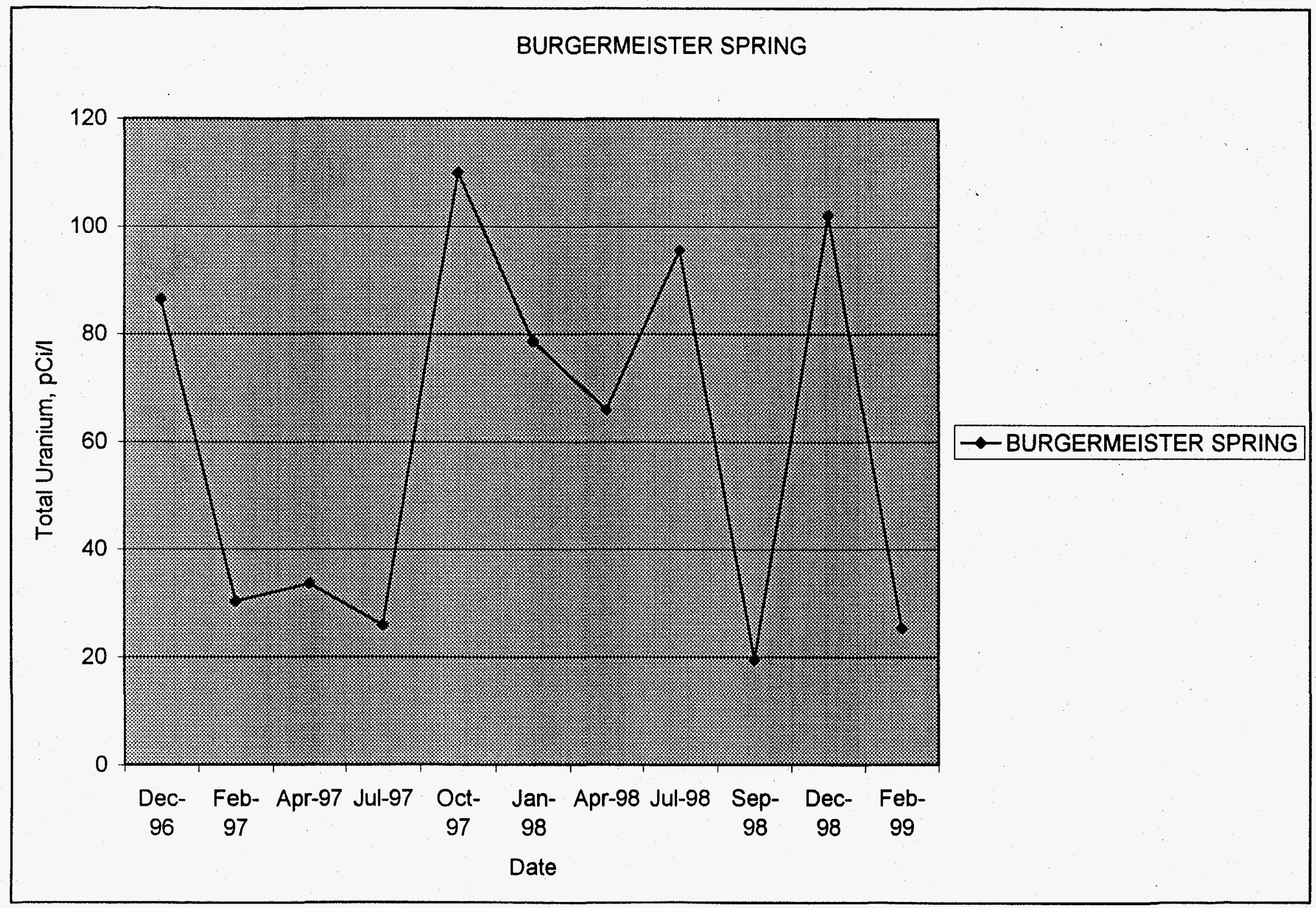


SPRINGS

DATA MERGED DURING FIRST QUARTER, 1999

\begin{tabular}{|c|c|c|c|c|c|c|c|}
\hline WSSRAP_ID & DATE_SAM & PARAMETER & CONC & DL & UNITS & & МETHOD \\
\hline 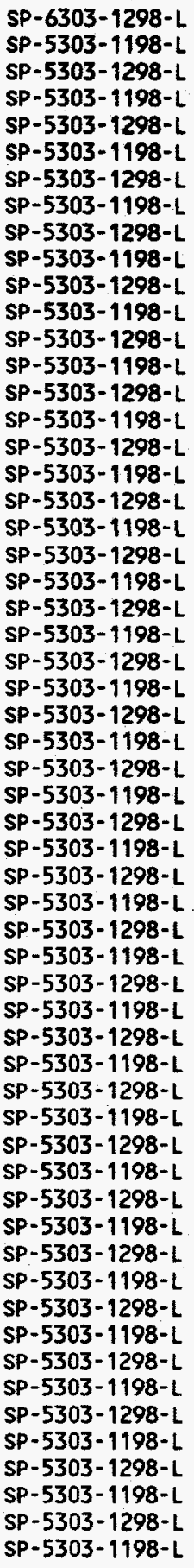 & $\begin{array}{l}12 / 14 / 98 \\
11 / 18 / 98 \\
12 / 16 / 98 \\
11 / 18 / 98 \\
12 / 16 / 98 \\
11 / 18 / 98 \\
12 / 16 / 98 \\
11 / 18 / 98 \\
12 / 16 / 98 \\
11 / 18 / 98 \\
12 / 16 / 98 \\
11 / 18 / 98 \\
12 / 16 / 98 \\
11 / 18 / 98 \\
12 / 16 / 98 \\
11 / 18 / 98 \\
12 / 16 / 98 \\
11 / 18 / 98 \\
12 / 16 / 98 \\
11 / 18 / 98 \\
12 / 16 / 98 \\
11 / 18 / 98 \\
12 / 16 / 98 \\
11 / 18 / 98 \\
12 / 16 / 98 \\
11 / 18 / 98 \\
12 / 16 / 98 \\
11 / 18 / 98 \\
12 / 16 / 98 \\
11 / 18 / 98 \\
12 / 16 / 98 \\
11 / 18 / 98 \\
12 / 16 / 98 \\
11 / 18 / 98 \\
12 / 16 / 98 \\
11 / 18 / 98 \\
12 / 16 / 98 \\
11 / 18 / 98 \\
12 / 16 / 98 \\
11 / 18 / 98 \\
12 / 16 / 98 \\
11 / 18 / 98 \\
12 / 16 / 98 \\
11 / 18 / 98 \\
12 / 16 / 98 \\
11 / 18 / 98 \\
12 / 16 / 98 \\
11 / 18 / 98 \\
12 / 16 / 98 \\
11 / 18 / 98 \\
12 / 16 / 98 \\
11 / 18 / 98 \\
12 / 16 / 98 \\
11 / 18 / 98 \\
12 / 16 / 98 \\
11 / 18 / 98 \\
12 / 16 / 98 \\
11 / 18 / 98\end{array}$ & 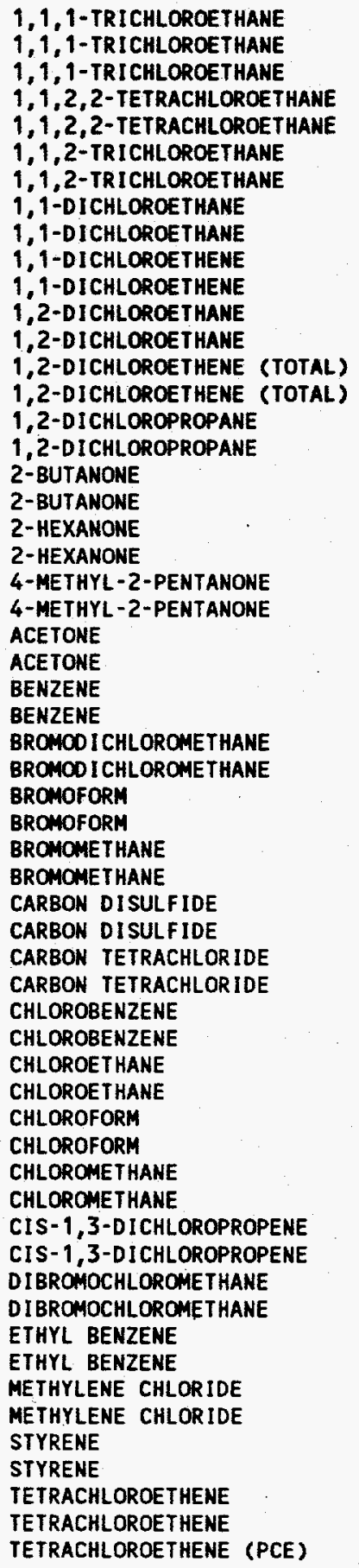 & $\begin{array}{l}\text { ND } \\
\text { ND } \\
\text { ND } \\
\text { ND } \\
\text { ND } \\
\text { ND } \\
\text { ND } \\
\text { ND } \\
\text { ND } \\
\text { ND } \\
\text { ND } \\
\text { ND } \\
\text { ND } \\
\text { ND } \\
\text { ND } \\
\text { ND } \\
\text { ND } \\
\text { ND } \\
\text { ND } \\
\text { ND } \\
\text { ND } \\
\text { ND } \\
\text { ND } \\
\text { ND } \\
\text { ND } \\
\text { ND } \\
\text { ND } \\
\text { ND } \\
\text { ND } \\
\text { ND } \\
\text { ND } \\
\text { ND } \\
\text { ND } \\
\text { ND } \\
\text { ND } \\
\text { ND } \\
\text { ND } \\
\text { ND } \\
\text { ND } \\
\text { ND } \\
\text { ND } \\
\text { ND } \\
\text { ND } \\
\text { ND } \\
\text { ND } \\
\text { ND } \\
\text { ND } \\
\text { ND } \\
\text { ND } \\
\text { ND } \\
\text { ND } \\
\text { (3.9) } \\
\text { (2) } \\
\text { ND } \\
\text { ND } \\
\text { ND } \\
\text { ND } \\
\text { ND }\end{array}$ & $\begin{array}{l}10 \\
10.0 \\
10 \\
10.0 \\
10 \\
10.0 \\
10 \\
10.0 \\
10 \\
10.0 \\
10 \\
10.0 \\
10 \\
10.0 \\
10 \\
10.0 \\
10 \\
10.0 \\
10 \\
10.0 \\
10 \\
10.0 \\
10 \\
10.0 \\
10 \\
10.0 \\
10 \\
10.0 \\
10 \\
10.0 \\
10 \\
10.0 \\
10 \\
10.0 \\
10 \\
10.0 \\
10 \\
10.0 \\
10 \\
10.0 \\
10 \\
10.0 \\
10 \\
10.0 \\
10 \\
10.0 \\
10 \\
10.0 \\
10 \\
10.0 \\
10 \\
10.0 \\
10 \\
10.0 \\
10 \\
1.0 \\
10 \\
1.0\end{array}$ & 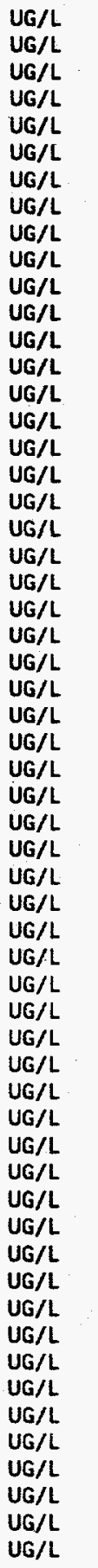 & $\begin{array}{l}E P A \\
E P A \\
E P A \\
E P A \\
E P A \\
E P A \\
E P A \\
E P A \\
E P A \\
E P A \\
E P A \\
E P A \\
E P A \\
E P A \\
E P A \\
E P A \\
E P A \\
E P A \\
E P A \\
E P A \\
E P A \\
E P A \\
E P A \\
E P A \\
E P A \\
E P A \\
E P A \\
E P A \\
E P A \\
E P A \\
E P A \\
E P A \\
E P A \\
E P A \\
E P A \\
E P A \\
E P A \\
E P A \\
E P A \\
E P A \\
E P A \\
E P A \\
E P A \\
E P A \\
E P A \\
E P A \\
E P A \\
E P A \\
E P A \\
E P A \\
E P A \\
E P A \\
E P A \\
\text { EA } \\
\text { EA } \\
\text { EA } \\
\text { EA } \\
\text { EA }\end{array}$ & $\begin{array}{l}\text { CLP } \\
8260 B \\
\text { CLP } \\
8260 B \\
\text { CLP } \\
8260 B \\
\text { CLP } \\
8260 B \\
\text { CLP } \\
8260 B \\
\text { CLP } \\
8260 B \\
\text { CLP } \\
8260 B \\
\text { CLP } \\
8260 B \\
\text { CLP } \\
8260 B \\
\text { CLP } \\
8260 B \\
\text { CLP } \\
8260 B \\
\text { CLP } \\
8260 B \\
\text { CLP } \\
8260 B \\
\text { CLP } \\
8260 B \\
\text { CLP } \\
8260 B \\
\text { CLP } \\
8260 B \\
\text { CLP } \\
8260 B \\
\text { CLP } \\
8260 B \\
\text { CLP } \\
8260 B \\
\text { CLP } \\
8260 B \\
\text { CLP } \\
8260 B \\
C L P \\
8260 B \\
\text { CLP } \\
8260 B \\
\text { CLP } \\
8260 B \\
\text { CLP } \\
8260 B \\
\text { CLP } \\
8260 B \\
\text { CLP } \\
8260 B \\
\text { CLP } \\
8260 B \\
\text { CLP } \\
8260 B\end{array}$ \\
\hline
\end{tabular}




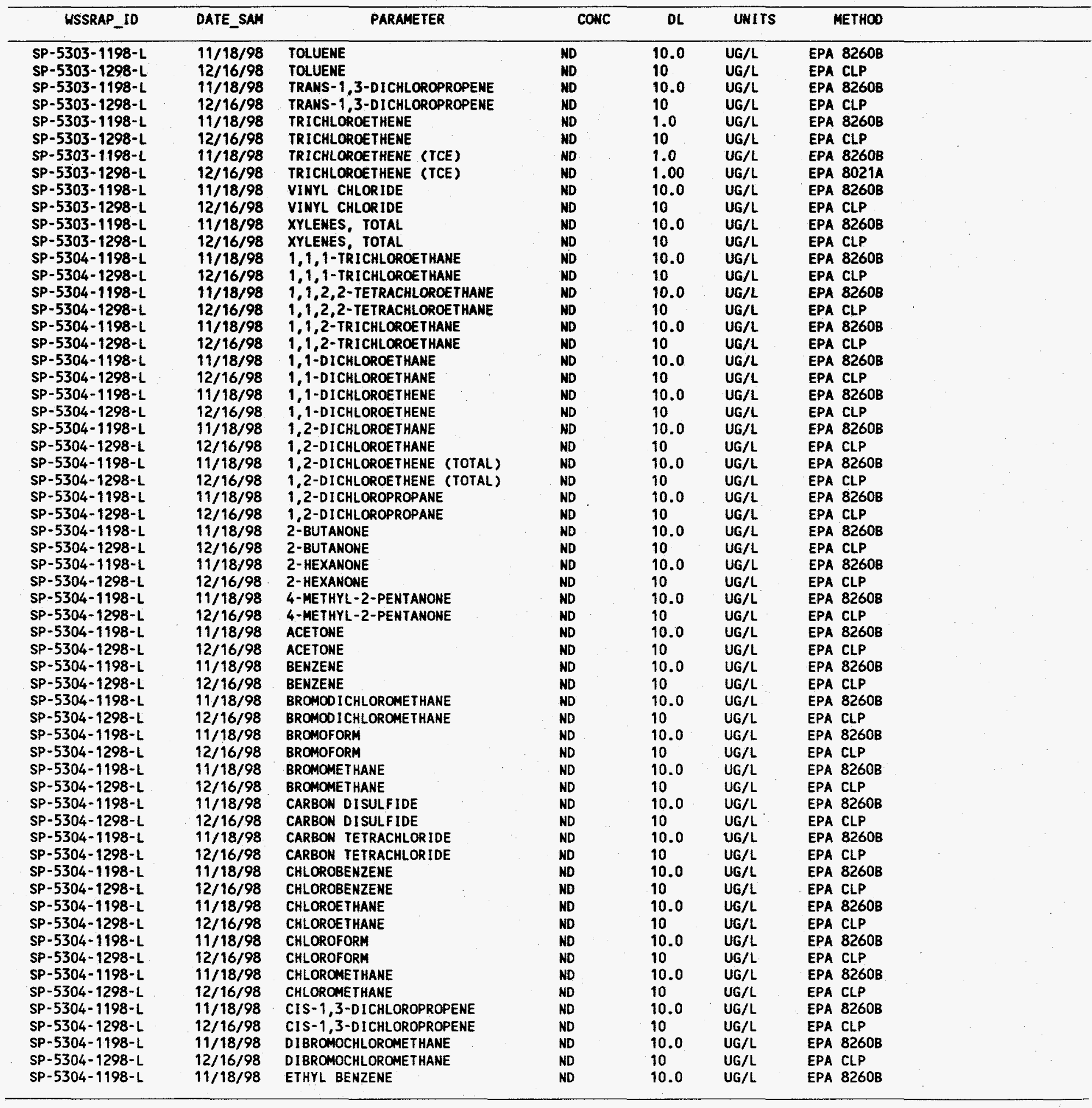




\begin{tabular}{|c|c|c|c|c|c|c|c|}
\hline WSSRAP_ID & DATE_SAM & PARAMETER & CONC & DL & UNITS & METHOO & \\
\hline 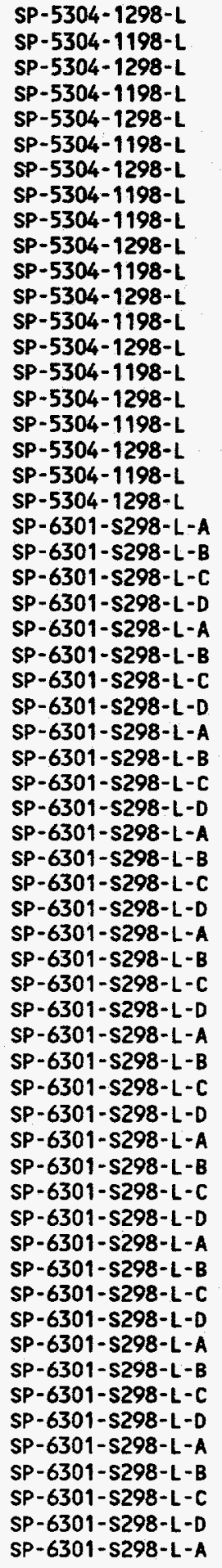 & $\begin{array}{l}12 / 16 / 98 \\
11 / 18 / 98 \\
12 / 16 / 98 \\
11 / 18 / 98 \\
12 / 16 / 98 \\
11 / 18 / 98 \\
12 / 16 / 98 \\
11 / 18 / 98 \\
11 / 18 / 98 \\
12 / 16 / 98 \\
11 / 18 / 98 \\
12 / 16 / 98 \\
11 / 18 / 98 \\
12 / 16 / 98 \\
11 / 18 / 98 \\
12 / 16 / 98 \\
11 / 18 / 98 \\
12 / 16 / 98 \\
11 / 18 / 98 \\
12 / 16 / 98 \\
12 / 12 / 98 \\
12 / 12 / 98 \\
12 / 12 / 98 \\
12 / 12 / 98 \\
12 / 12 / 98 \\
12 / 12 / 98 \\
12 / 12 / 98 \\
12 / 12 / 98 \\
12 / 12 / 98 \\
12 / 12 / 98 \\
12 / 12 / 98 \\
12 / 12 / 98 \\
12 / 12 / 98 \\
12 / 12 / 98 \\
12 / 12 / 98 \\
12 / 12 / 98 \\
12 / 12 / 98 \\
12 / 12 / 98 \\
12 / 12 / 98 \\
12 / 12 / 98 \\
12 / 12 / 98 \\
12 / 12 / 98 \\
12 / 12 / 98 \\
12 / 12 / 98 \\
12 / 12 / 98 \\
12 / 12 / 98 \\
12 / 12 / 98 \\
12 / 12 / 98 \\
12 / 12 / 98 \\
12 / 12 / 98 \\
12 / 12 / 98 \\
12 / 12 / 98 \\
12 / 12 / 98 \\
12 / 12 / 98 \\
12 / 12 / 98 \\
12 / 12 / 98 \\
12 / 12 / 98 \\
12 / 12 / 98 \\
12 / 12 / 98 \\
12 / 12 / 98 \\
12 / 12 / 98\end{array}$ & $\begin{array}{l}\text { ETHYL BENZENE } \\
\text { METHYLENE CHLORIDE } \\
\text { METHYLENE CHLORIDE } \\
\text { STYRENE } \\
\text { STYRENE } \\
\text { TETRACHLOROETHENE } \\
\text { TETRACHLOROETHENE } \\
\text { TETRACHLOROETHENE (PCE) } \\
\text { TOLUENE } \\
\text { TOLUENE } \\
\text { TRANS-1,3-DICHLOROPROPENE } \\
\text { TRANS-1,3-DICHLOROPROPENE } \\
\text { TRICHLOROETHENE } \\
\text { TRICHLOROETHENE } \\
\text { TRICHLOROETHENE (TCE) } \\
\text { TRICHLOROETHENE (TCE) } \\
\text { VINYL CHLORIDE } \\
\text { VINYL CHLORIDE } \\
\text { XYLENES, TOTAL } \\
\text { XYLENES, TOTAL } \\
\text { CHLORIDE } \\
\text { CHLORIDE } \\
\text { CHLORIDE } \\
\text { CHLORIDE } \\
\text { FLUORIDE } \\
\text { FLUORIDE } \\
\text { FLUORIDE } \\
\text { FLUORIDE } \\
\text { NITRATE-N } \\
\text { NITRATE-H } \\
\text { NITRATE-N } \\
\text { NITRATE-N } \\
\text { SULFATE } \\
\text { SULFATE } \\
\text { SULFATE } \\
\text { SULFATE } \\
\text { ALUMINUM } \\
\text { ALUMINUM } \\
\text { ALUMINUM } \\
\text { ALUMINUM } \\
\text { ANTIMONY } \\
\text { ANTIMONY } \\
\text { ANTIMONY } \\
\text { ANTIMONY } \\
\text { ARSENIC } \\
\text { ARSENIC } \\
\text { ARSENIC } \\
\text { ARSENIC } \\
\text { BARIUM } \\
\text { BARIUM } \\
\text { BARIUM } \\
\text { BARIUM } \\
\text { BERYLLIUM } \\
\text { BERYLLIUM } \\
\text { BERYLLIUM } \\
\text { BERYLLIUM } \\
\text { CADMIUM } \\
\text { CADMIUM } \\
\text { CADMIUM } \\
\text { CADMIUM } \\
\text { CALCIUM } \\
\text { CAUM }\end{array}$ & $\begin{array}{l}\text { ND } \\
\text { ND } \\
\text { C2) } \\
\text { ND } \\
\text { ND } \\
\text { ND } \\
\text { ND } \\
\text { ND } \\
\text { ND } \\
\text { ND } \\
\text { ND } \\
\text { ND } \\
\text { ND } \\
\text { ND } \\
\text { ND } \\
\text { ND } \\
\text { ND } \\
\text { ND } \\
\text { ND } \\
\text { ND } \\
19.0 \\
12.3 \\
13.0 \\
11.7 \\
\text { ND } \\
\text { ND } \\
\text { ND } \\
\text { ND } \\
9.2 \\
9.3 \\
9.2 \\
9.2 \\
45.0 \\
43.8 \\
44.0 \\
44.0 \\
243 \\
277 \\
272 \\
357 \\
\text { ND } \\
\text { ND } \\
\text { ND } \\
\text { ND } \\
\text { ND } \\
\text { ND } \\
\text { ND } \\
\text { ND } \\
124 \\
126 \\
128 \\
130 \\
\text { ND } \\
\text { ND } \\
\text { ND } \\
\text { ND } \\
\text { ND } \\
\text { ND } \\
\text { ND } \\
\text { ND } \\
72100\end{array}$ & $\begin{array}{l}10 \\
10.0 \\
10 \\
10.0 \\
10 \\
1.0 \\
10 \\
1.0 \\
10.0 \\
10 \\
10.0 \\
10 \\
1.0 \\
10 \\
1.0 \\
1.00 \\
10.0 \\
10 \\
10.0 \\
10 \\
2.50 \\
0.50 \\
0.50 \\
0.50 \\
0.50 \\
0.50 \\
0.50 \\
0.50 \\
0.20 \\
0.20 \\
0.20 \\
0.20 \\
2.50 \\
2.50 \\
2.50 \\
2.50 \\
14.2 \\
14.2 \\
14.2 \\
14.2 \\
3.50 \\
3.50 \\
3.50 \\
3.50 \\
2.80 \\
2.80 \\
2.80 \\
2.80 \\
0.30 \\
0.30 \\
0.30 \\
0.30 \\
0.10 \\
0.10 \\
0.10 \\
0.10 \\
0.40 \\
0.40 \\
0.40 \\
0.40 \\
4.70\end{array}$ & 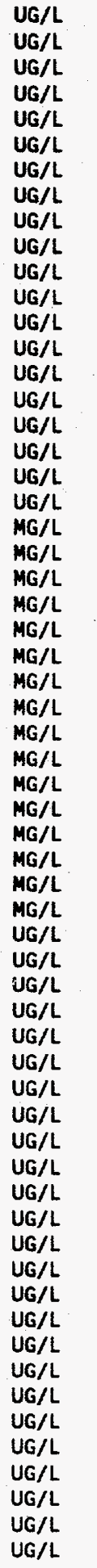 & 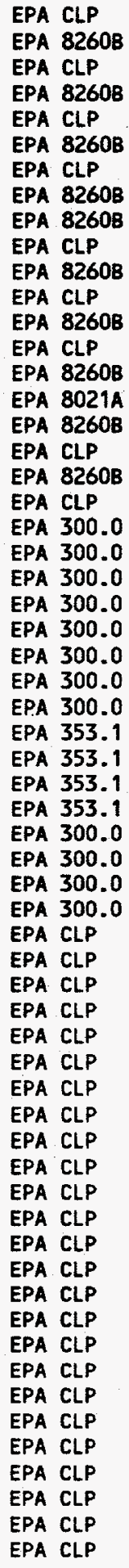 & - \\
\hline
\end{tabular}




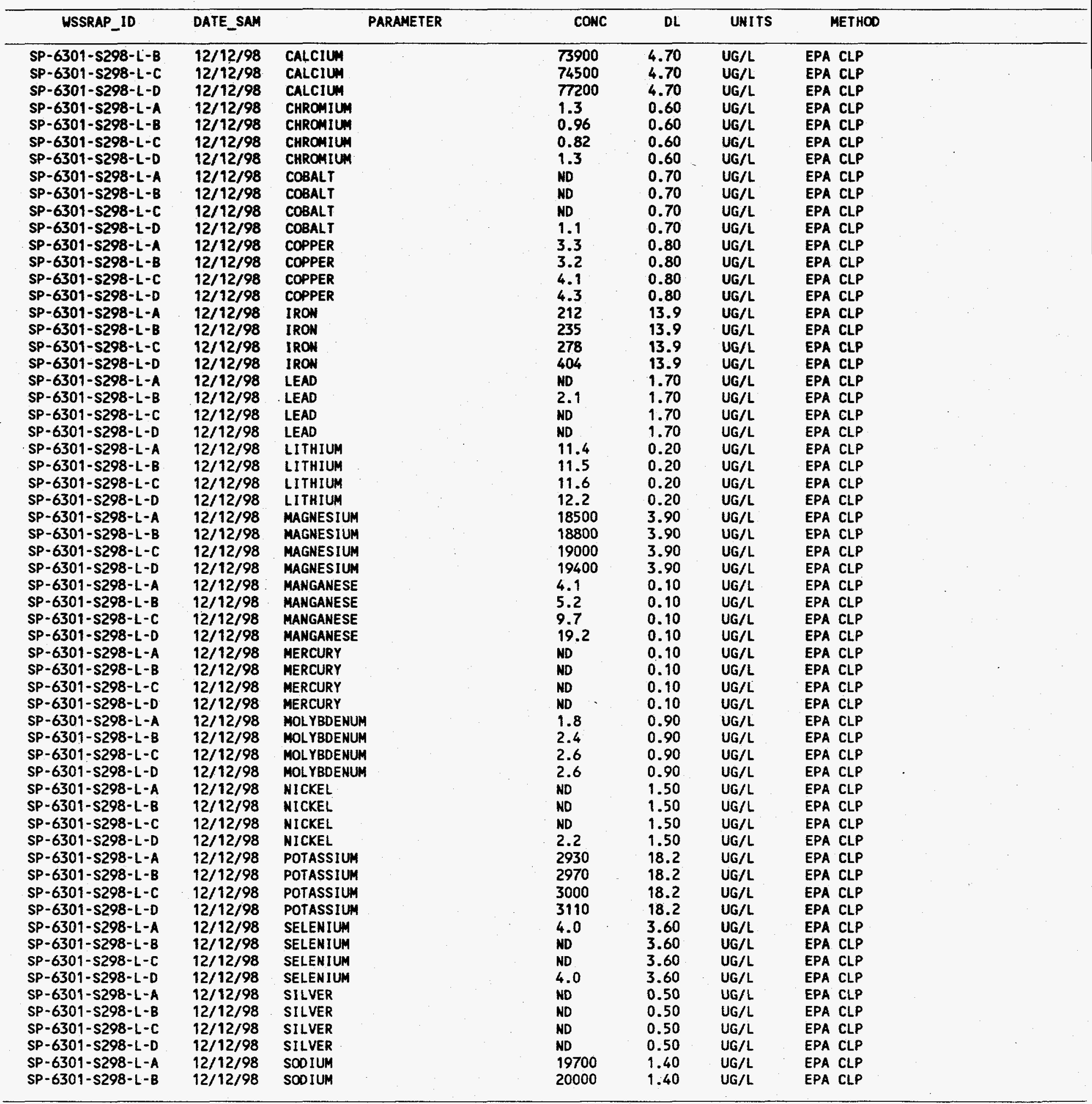




\begin{tabular}{|c|c|c|c|c|c|c|}
\hline USSRAP_ID & DATE_SAM & PARAMETER & CONC & $D L$ & UNITS & METHOD \\
\hline 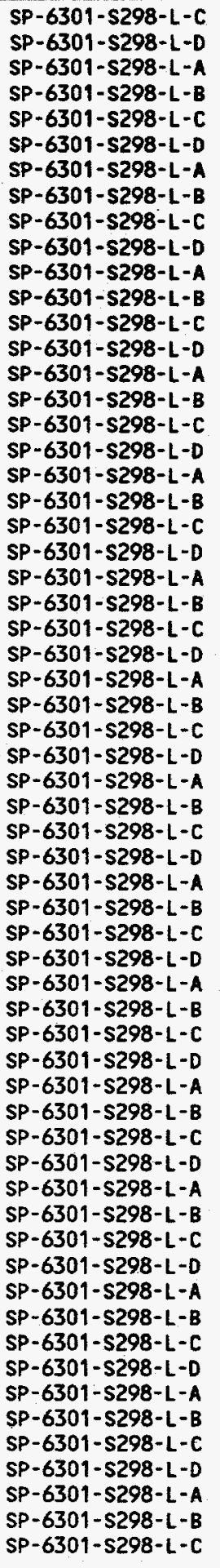 & $\begin{array}{l}12 / 12 / 98 \\
12 / 12 / 98 \\
12 / 12 / 98 \\
12 / 12 / 98 \\
12 / 12 / 98 \\
12 / 12 / 98 \\
12 / 12 / 98 \\
12 / 12 / 98 \\
12 / 12 / 98 \\
12 / 12 / 98 \\
12 / 12 / 98 \\
12 / 12 / 98 \\
12 / 12 / 98 \\
12 / 12 / 98 \\
12 / 12 / 98 \\
12 / 12 / 98 \\
12 / 12 / 98 \\
12 / 12 / 98 \\
12 / 12 / 98 \\
12 / 12 / 98 \\
12 / 12 / 98 \\
12 / 12 / 98 \\
12 / 12 / 98 \\
12 / 12 / 98 \\
12 / 12 / 98 \\
12 / 12 / 98 \\
12 / 12 / 98 \\
12 / 12 / 98 \\
12 / 12 / 98 \\
12 / 12 / 98 \\
12 / 12 / 98 \\
12 / 12 / 98 \\
12 / 12 / 98 \\
12 / 12 / 98 \\
12 / 12 / 98 \\
12 / 12 / 98 \\
12 / 12 / 98 \\
12 / 12 / 98 \\
12 / 12 / 98 \\
12 / 12 / 98 \\
12 / 12 / 98 \\
12 / 12 / 98 \\
12 / 12 / 98 \\
12 / 12 / 98 \\
12 / 12 / 98 \\
12 / 12 / 98 \\
12 / 12 / 98 \\
12 / 12 / 98 \\
12 / 12 / 98 \\
12 / 12 / 98 \\
12 / 12 / 98 \\
12 / 12 / 98 \\
12 / 12 / 98 \\
12 / 12 / 98 \\
12 / 12 / 98 \\
12 / 12 / 98 \\
12 / 12 / 98 \\
12 / 12 / 98 \\
12 / 12 / 98 \\
12 / 12 / 98 \\
12 / 12 / 98\end{array}$ & 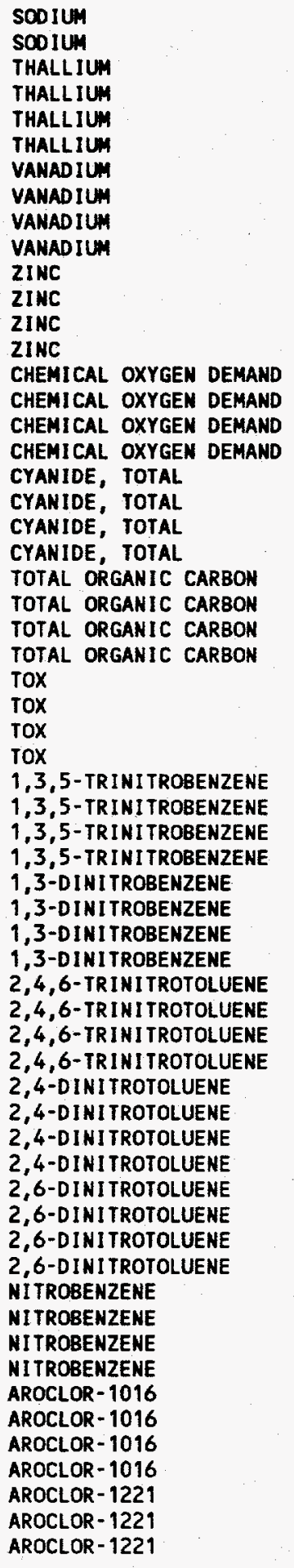 & 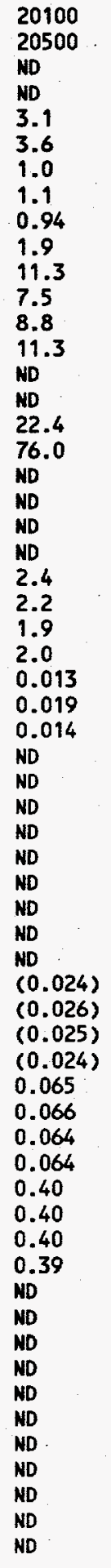 & $\begin{array}{l}1.40 \\
1.40 \\
2.80 \\
2.80 \\
2.80 \\
2.80 \\
0.60 \\
0.60 \\
0.60 \\
0.60 \\
0.60 \\
0.60 \\
0.60 \\
0.60 \\
5.00 \\
5.00 \\
5.00 \\
5.00 \\
5.00 \\
5.00 \\
5.00 \\
5.00 \\
0.50 \\
0.50 \\
0.50 \\
0.50 \\
0.012 \\
0.012 \\
0.012 \\
0.012 \\
0.030 \\
0.030 \\
0.030 \\
0.030 \\
0.090 \\
0.090 \\
0.090 \\
0.090 \\
0.030 \\
0.030 \\
0.030 \\
0.030 \\
0.030 \\
0.030 \\
0.030 \\
0.030 \\
0.010 \\
0.010 \\
0.010 \\
0.010 \\
0.030 \\
0.030 \\
0.030 \\
0.030 \\
1.00 \\
1.00 \\
1.00 \\
1.00 \\
2.00 \\
2.00 \\
2.00\end{array}$ & $\begin{array}{l}U G / L \\
U G / L \\
U G / L \\
U G / L \\
U G / L \\
U G / L \\
U G / L \\
U G / L \\
U G / L \\
U G / L \\
U G / L \\
U G / L \\
U G / L \\
U G / L \\
M G / L \\
M G / L \\
M G / L \\
M G / L \\
U G / L \\
U G / L \\
U G / L \\
U G / L \\
M G / L \\
M G / L \\
M G / L \\
M G / L \\
M G / L \\
M G / L \\
M G / L \\
M G / L \\
U G / L \\
U G / L \\
U G / L \\
U G / L \\
U G / L \\
U G / L \\
U G / L \\
U G / L \\
U G / L \\
U G / L \\
U G / L \\
U G / L \\
U G / L \\
U G / L \\
U G / L \\
U G / L \\
U G / L \\
U G / L \\
U G / L \\
U G / L \\
U G / L \\
U G / L \\
U G / L \\
U G / L \\
U G / L \\
U G / L \\
U G / L \\
U G / L \\
U G / L \\
U G / L \\
U G / L \\
U\end{array}$ & $\begin{array}{l}\text { EPA CLP } \\
\text { EPA CLP } \\
\text { EPA CLP } \\
\text { EPA CLP } \\
\text { EPA CLP } \\
\text { EPA CLP } \\
\text { EPA CLP } \\
\text { EPA CLP } \\
\text { EPA CLP } \\
\text { EPA CLP } \\
\text { EPA CLP } \\
\text { EPA CLP } \\
\text { EPA CLP } \\
\text { EPA CLP } \\
\text { EPA } 410.0 \\
\text { EPA } 410.0 \\
\text { EPA } 410.0 \\
\text { EPA } 410.0 \\
\text { EPA CLP } \\
\text { EPA CLP } \\
\text { EPA CLP } \\
\text { EPA CLP } \\
\text { EPA } 9060 \\
\text { EPA } 9060 \\
\text { EPA } 9060 \\
\text { EPA } 9060 \\
\text { EPA } 9020 \\
\text { EPA } 9020 \\
\text { EPA } 9020 \\
\text { EPA } 9020 \\
\text { USATHAMA } \\
\text { USATHAMA } \\
\text { USATHAMA } \\
\text { USATHAMA } \\
\text { USATHAMA } \\
\text { USATHAMA } \\
\text { USATHAMA } \\
\text { USATHAMA } \\
\text { USATHAMA } \\
\text { USATHAMA } \\
\text { USATHAMA } \\
\text { USATHAMA } \\
\text { USATHAMA } \\
\text { USATHAMA } \\
\text { USATHAMA } \\
\text { USATHAMA } \\
\text { USATHAMA } \\
\text { USATHAMA } \\
\text { USATHAMA } \\
\text { USATHAMA } \\
\text { USATHAMA } \\
\text { USATHAMA } \\
\text { USATHAMA } \\
\text { USATHAMA } \\
\text { EPA } 8081 \\
\text { EPA } 8081 \\
\text { EPA } 8081 \\
\text { EPA } 8081 \\
\text { EPA } 8081 \\
\text { EPA } 8081 \\
\text { EPA } 8081\end{array}$ \\
\hline
\end{tabular}




\begin{tabular}{|c|c|c|c|c|c|c|}
\hline WSSRAP_ID & DATE_SAM & PARAMETER & CONC & DL & UNITS & METHOD \\
\hline 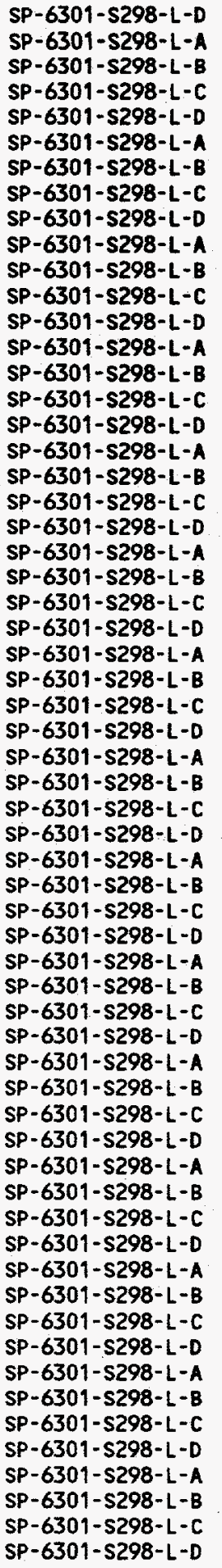 & $\begin{array}{l}12 / 12 / 98 \\
12 / 12 / 98 \\
12 / 12 / 98 \\
12 / 12 / 98 \\
12 / 12 / 98 \\
12 / 12 / 98 \\
12 / 12 / 98 \\
12 / 12 / 98 \\
12 / 12 / 98 \\
12 / 12 / 98 \\
12 / 12 / 98 \\
12 / 12 / 98 \\
12 / 12 / 98 \\
12 / 12 / 98 \\
12 / 12 / 98 \\
12 / 12 / 98 \\
12 / 12 / 98 \\
12 / 12 / 98 \\
12 / 12 / 98 \\
12 / 12 / 98 \\
12 / 12 / 98 \\
12 / 12 / 98 \\
12 / 12 / 98 \\
12 / 12 / 98 \\
12 / 12 / 98 \\
12 / 12 / 98 \\
12 / 12 / 98 \\
12 / 12 / 98 \\
12 / 12 / 98 \\
12 / 12 / 98 \\
12 / 12 / 98 \\
12 / 12 / 98 \\
12 / 12 / 98 \\
12 / 12 / 98 \\
12 / 12 / 98 \\
12 / 12 / 98 \\
12 / 12 / 98 \\
12 / 12 / 98 \\
12 / 12 / 98 \\
12 / 12 / 98 \\
12 / 12 / 98 \\
12 / 12 / 98 \\
12 / 12 / 98 \\
12 / 12 / 98 \\
12 / 12 / 98 \\
12 / 12 / 98 \\
12 / 12 / 98 \\
12 / 12 / 98 \\
12 / 12 / 98 \\
12 / 12 / 98 \\
12 / 12 / 98 \\
12 / 12 / 98 \\
12 / 12 / 98 \\
12 / 12 / 98 \\
12 / 12 / 98 \\
12 / 12 / 98 \\
12 / 12 / 98 \\
12 / 12 / 98 \\
12 / 12 / 98 \\
12 / 12 / 98 \\
12 / 12 / 98\end{array}$ & $\begin{array}{l}\text { AROCLOR-1221 } \\
\text { AROCLOR-1232 } \\
\text { AROCLOR-1232 } \\
\text { AROCLOR-1232 } \\
\text { AROCLOR-1232 } \\
\text { AROCLOR-1242 } \\
\text { AROCLOR-1242 } \\
\text { AROCLOR-1242 } \\
\text { AROCLOR-1242 } \\
\text { AROCLOR-1248 } \\
\text { AROCLOR-1248 } \\
\text { AROCLOR-1248 } \\
\text { AROCLOR-1248 } \\
\text { AROCLOR-1254 } \\
\text { AROCLOR-1254 } \\
\text { AROCLOR-1254 } \\
\text { AROCLOR-1254 } \\
\text { AROCLOR-1260 } \\
\text { AROCLOR-1260 } \\
\text { AROCLOR-1260 } \\
\text { AROCLOR-1260 } \\
\text { RADIUH-226 } \\
\text { RADIUM-226 } \\
\text { RADIUM-226 } \\
\text { RADIUM-226 } \\
\text { RADIUH-228 } \\
\text { RADIUM-228 } \\
\text { RADIUM-228 } \\
\text { RADIUM-228 } \\
\text { THORIUM-228 } \\
\text { THORIUM-228 } \\
\text { THORIUM-228 } \\
\text { THORIUM-228 } \\
\text { THORIUM-230 } \\
\text { THORIUM-230 } \\
\text { THORIUM-230 } \\
\text { THORIUM-230 } \\
\text { THORIUM-232 } \\
\text { THORIUM-232 } \\
\text { THORIUM-232 } \\
\text { THORIUM-232 } \\
\text { URANIUM, TOTAL } \\
\text { URANIUM, TOTAL } \\
\text { URANIUM, TOTAL } \\
\text { URANIUM, TOTAL } \\
\text { ACENAPHTHENE } \\
\text { ACENAPHTHENE } \\
\text { ACENAPHTHENE } \\
\text { ACENAPHTHENE } \\
\text { ACENAPHTHYLENE } \\
\text { ACENAPHTHYLENE } \\
\text { ACENAPHTHYLENE } \\
\text { ACENAPHTHYLENE } \\
\text { ANTHRACENE } \\
\text { ANTHRACENE } \\
\text { ANTHRACENE } \\
\text { ANTHRACENE } \\
\text { BENZOCA)ANTHRACENE } \\
\text { BENZO(A)ANTHRACENE } \\
\text { BENZO(A)ANTHRACENE } \\
\text { BENZO(A)ANTHRACENE }\end{array}$ & $\begin{array}{l}\text { ND } \\
\text { ND } \\
\text { ND } \\
\text { ND } \\
\text { ND } \\
\text { ND } \\
\text { ND } \\
\text { ND } \\
\text { ND } \\
\text { ND } \\
\text { ND } \\
\text { ND } \\
\text { ND } \\
\text { ND } \\
\text { ND } \\
\text { ND } \\
\text { ND } \\
\text { ND } \\
\text { ND } \\
\text { ND } \\
\text { ND } \\
0.297 \\
(0.068) \\
(0.089) \\
(0.093) \\
\text { ND } \\
\text { ND } \\
\text { ND } \\
\text { ND } \\
(0.084) \\
0.383 \\
0.383 \\
0.218 \\
0.363 \\
0.457 \\
0.336 \\
0.298 \\
(0.112) \\
0.184 \\
0.077 \\
0.077 \\
85.3 \\
83.3 \\
154 \\
85.3 \\
\text { ND } \\
\text { ND } \\
\text { ND } \\
\text { ND } \\
\text { ND } \\
\text { ND } \\
\text { ND } \\
\text { ND } \\
\text { ND } \\
\text { ND } \\
\text { ND } \\
\text { ND } \\
\text { ND } \\
\text { ND } \\
\text { ND } \\
\text { ND }\end{array}$ & $\begin{array}{l}2.00 \\
1.00 \\
1.00 \\
1.00 \\
1.00 \\
1.00 \\
1.00 \\
1.00 \\
1.00 \\
1.00 \\
1.00 \\
1.00 \\
1.00 \\
1.00 \\
1.00 \\
1.00 \\
1.00 \\
1.00 \\
1.00 \\
1.00 \\
1.00 \\
0.164 \\
0.220 \\
0.219 \\
0.175 \\
0.469 \\
0.469 \\
0.469 \\
0.469 \\
0.151 \\
0.148 \\
0.120 \\
0.095 \\
0.127 \\
0.129 \\
0.047 \\
0.049 \\
0.151 \\
0.129 \\
0.062 \\
0.041 \\
0.677 \\
0.677 \\
0.677 \\
0.677 \\
18.0 \\
18.0 \\
18.0 \\
18.0 \\
23.0 \\
23.0 \\
23.0 \\
23.0 \\
6.60 \\
6.60 \\
6.60 \\
6.60 \\
0.13 \\
0.13 \\
0.13 \\
0.13\end{array}$ & $\begin{array}{l}U G / L \\
U G / L \\
U G / L \\
U G / L \\
U G / L \\
U G / L \\
U G / L \\
U G / L \\
U G / L \\
U G / L \\
U G / L \\
U G / L \\
U G / L \\
U G / L \\
U G / L \\
U G / L \\
U G / L \\
U G / L \\
U G / L \\
U G / L \\
U G / L \\
P C I / L \\
P C I / L \\
P C I / L \\
P C I / L \\
P C I / L \\
P C I / L \\
P C I / L \\
P C I / L \\
P C I / L \\
P C I / L \\
P C I / L \\
P C I / L \\
P C I / L \\
P C I / L \\
P C I / L \\
P C I / L \\
P C I / L \\
P C I / L \\
P C I / L \\
P C I / L \\
P C I / L \\
P C I / L \\
P C I / L \\
P C I / L \\
U G / L \\
U G / L \\
U G / L \\
U G / L \\
U G / L \\
U G / L \\
U G / L \\
U G / L \\
U G / L \\
U G / L \\
U G / L \\
U G / L \\
U G / L \\
U G / L \\
U G / L \\
U G / L \\
\end{array}$ & 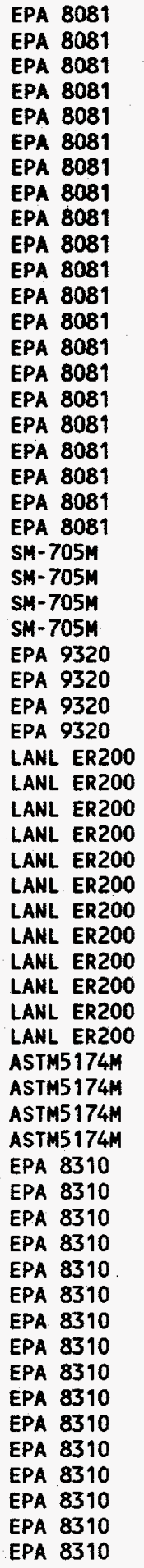 \\
\hline
\end{tabular}




\begin{tabular}{|c|c|c|c|c|c|c|}
\hline WSSRAP_ID & DATE_SAM & PARAMETER & CONC & DL & UNITS & METHOO \\
\hline 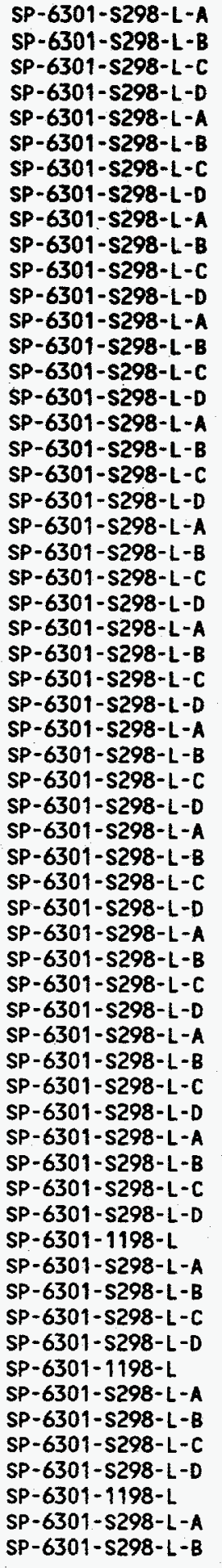 & $\begin{array}{l}12 / 12 / 98 \\
12 / 12 / 98 \\
12 / 12 / 98 \\
12 / 12 / 98 \\
12 / 12 / 98 \\
12 / 12 / 98 \\
12 / 12 / 98 \\
12 / 12 / 98 \\
12 / 12 / 98 \\
12 / 12 / 98 \\
12 / 12 / 98 \\
12 / 12 / 98 \\
12 / 12 / 98 \\
12 / 12 / 98 \\
12 / 12 / 98 \\
12 / 12 / 98 \\
12 / 12 / 98 \\
12 / 12 / 98 \\
12 / 12 / 98 \\
12 / 12 / 98 \\
12 / 12 / 98 \\
12 / 12 / 98 \\
12 / 12 / 98 \\
12 / 12 / 98 \\
12 / 12 / 98 \\
12 / 12 / 98 \\
12 / 12 / 98 \\
12 / 12 / 98 \\
12 / 12 / 98 \\
12 / 12 / 98 \\
12 / 12 / 98 \\
12 / 12 / 98 \\
12 / 12 / 98 \\
12 / 12 / 98 \\
12 / 12 / 98 \\
12 / 12 / 98 \\
12 / 12 / 98 \\
12 / 12 / 98 \\
12 / 12 / 98 \\
12 / 12 / 98 \\
12 / 112 / 98 \\
12 / 12 / 98 \\
12 / 12 / 98 \\
12 / 12 / 98 \\
12 / 12 / 98 \\
12 / 12 / 98 \\
12 / 12 / 98 \\
12 / 12 / 98 \\
11 / 18 / 98 \\
12 / 12 / 98 \\
12 / 12 / 98 \\
12 / 12 / 98 \\
12 / 12 / 98 \\
11 / 18 / 98 \\
12 / 12 / 98 \\
12 / 12 / 98 \\
12 / 12 / 98 \\
12 / 12 / 98 \\
11 / 18 / 98 \\
12 / 12 / 98 \\
12 / 12 / 98\end{array}$ & 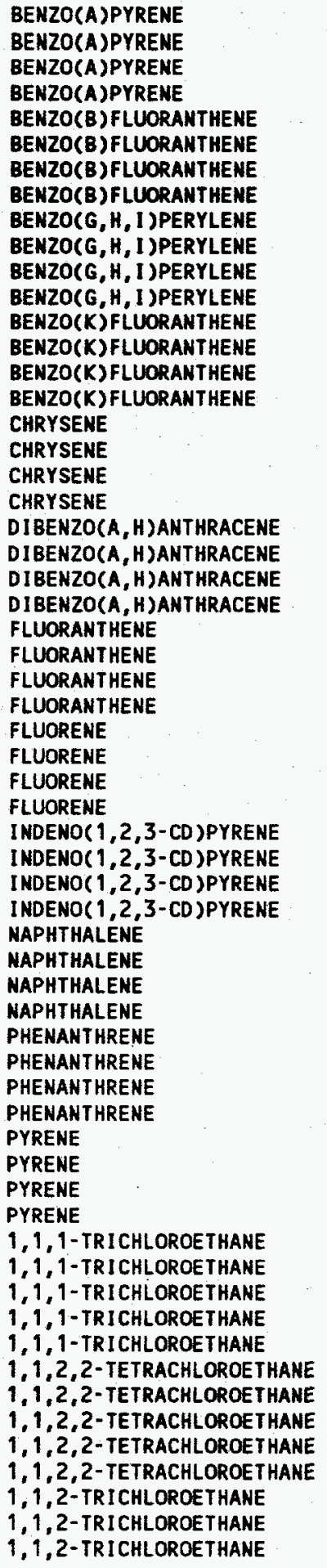 & $\begin{array}{l}\text { ND } \\
\text { ND } \\
\text { ND } \\
\text { ND } \\
\text { ND } \\
\text { ND } \\
\text { ND } \\
\text { ND } \\
\text { ND } \\
\text { ND } \\
\text { ND } \\
\text { ND } \\
\text { ND } \\
\text { ND } \\
\text { ND } \\
\text { ND } \\
\text { ND } \\
\text { ND } \\
\text { ND } \\
\text { ND } \\
\text { ND } \\
\text { ND } \\
\text { ND } \\
\text { ND } \\
\text { ND } \\
\text { ND } \\
\text { ND } \\
\text { ND } \\
\text { ND } \\
\text { ND } \\
\text { ND } \\
\text { ND } \\
\text { ND } \\
\text { ND } \\
\text { ND } \\
\text { ND } \\
\text { ND } \\
\text { ND } \\
\text { ND } \\
\text { ND } \\
\text { ND } \\
\text { ND } \\
\text { ND } \\
\text { ND } \\
\text { ND } \\
\text { ND } \\
\text { ND } \\
\text { ND } \\
\text { ND } \\
\text { ND } \\
\text { ND } \\
\text { ND } \\
\text { ND } \\
\text { ND } \\
\text { ND } \\
\text { ND } \\
\text { ND } \\
\text { ND } \\
\text { ND } \\
\text { ND } \\
\text { ND }\end{array}$ & $\begin{array}{l}0.23 \\
0.23 \\
0.23 \\
0.23 \\
0.18 \\
0.18 \\
0.18 \\
0.18 \\
0.76 \\
0.76 \\
0.76 \\
0.76 \\
0.17 \\
0.17 \\
0.17 \\
0.17 \\
1.50 \\
1.50 \\
1.50 \\
1.50 \\
0.30 \\
0.30 \\
0.30 \\
0.30 \\
2.10 \\
2.10 \\
2.10 \\
2.10 \\
2.10 \\
2.10 \\
2.10 \\
2.10 \\
0.43 \\
0.43 \\
0.43 \\
0.43 \\
18.0 \\
18.0 \\
18.0 \\
18.0 \\
6.40 \\
6.40 \\
6.40 \\
6.40 \\
2.70 \\
2.70 \\
2.70 \\
2.70 \\
10.0 \\
10 \\
10 \\
10 \\
10 \\
10.0 \\
10 \\
10 \\
10 \\
10 \\
10.0 \\
10 \\
10\end{array}$ & 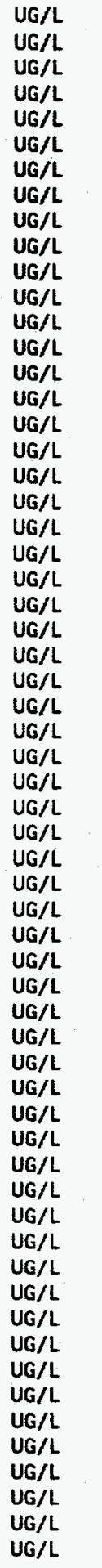 & 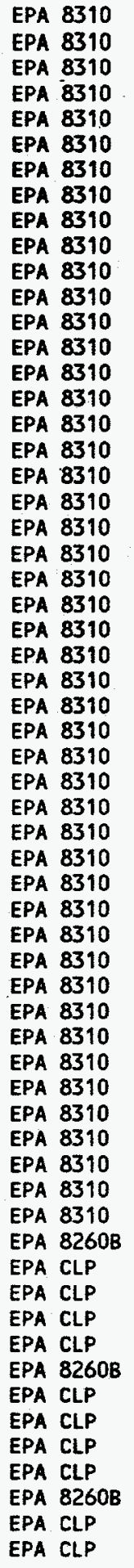 \\
\hline
\end{tabular}




\begin{tabular}{|c|c|c|c|c|c|c|}
\hline WSSRAP_ID & DATE_SAM & PARAMETER & CONC & $\dot{D L}$ & UNITS & METHOD \\
\hline 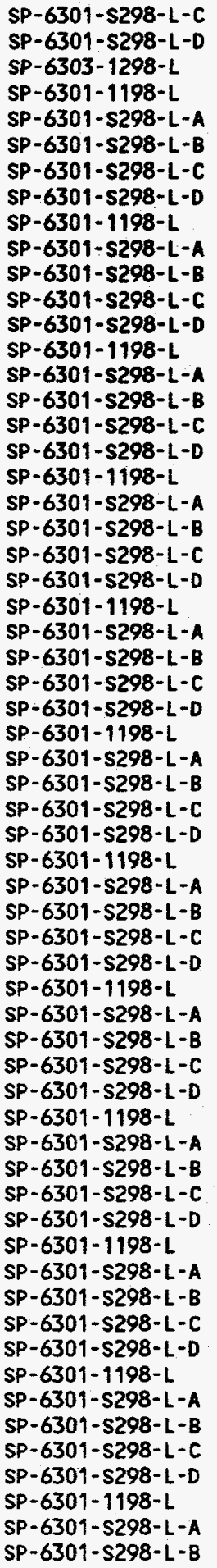 & $\begin{array}{l}12 / 12 / 98 \\
12 / 12 / 98 \\
12 / 14 / 98 \\
11 / 18 / 98 \\
12 / 12 / 98 \\
12 / 12 / 98 \\
12 / 12 / 98 \\
12 / 12 / 98 \\
11 / 18 / 98 \\
12 / 12 / 98 \\
12 / 12 / 98 \\
12 / 12 / 98 \\
12 / 12 / 98 \\
11 / 18 / 98 \\
12 / 12 / 98 \\
12 / 12 / 98 \\
12 / 12 / 98 \\
12 / 12 / 98 \\
11 / 18 / 98 \\
12 / 12 / 98 \\
12 / 12 / 98 \\
12 / 12 / 98 \\
12 / 12 / 98 \\
11 / 18 / 98 \\
12 / 12 / 98 \\
12 / 12 / 98 \\
12 / 12 / 98 \\
12 / 12 / 98 \\
11 / 18 / 98 \\
12 / 12 / 98 \\
12 / 12 / 98 \\
12 / 12 / 98 \\
12 / 12 / 98 \\
11 / 18 / 98 \\
12 / 12 / 98 \\
12 / 12 / 98 \\
12 / 12 / 98 \\
12 / 12 / 98 \\
11 / 18 / 98 \\
12 / 12 / 98 \\
12 / 12 / 98 \\
12 / 12 / 98 \\
12 / 12 / 98 \\
11 / 18 / 98 \\
12 / 12 / 98 \\
12 / 12 / 98 \\
12 / 12 / 98 \\
12 / 12 / 98 \\
11 / 18 / 98 \\
12 / 12 / 98 \\
12 / 12 / 98 \\
12 / 12 / 98 \\
12 / 12 / 98 \\
11 / 18 / 98 \\
12 / 12 / 98 \\
12 / 12 / 98 \\
12 / 12 / 98 \\
12 / 12 / 98 \\
11 / 18 / 98 \\
12 / 12 / 98 \\
12 / 12 / 98\end{array}$ & 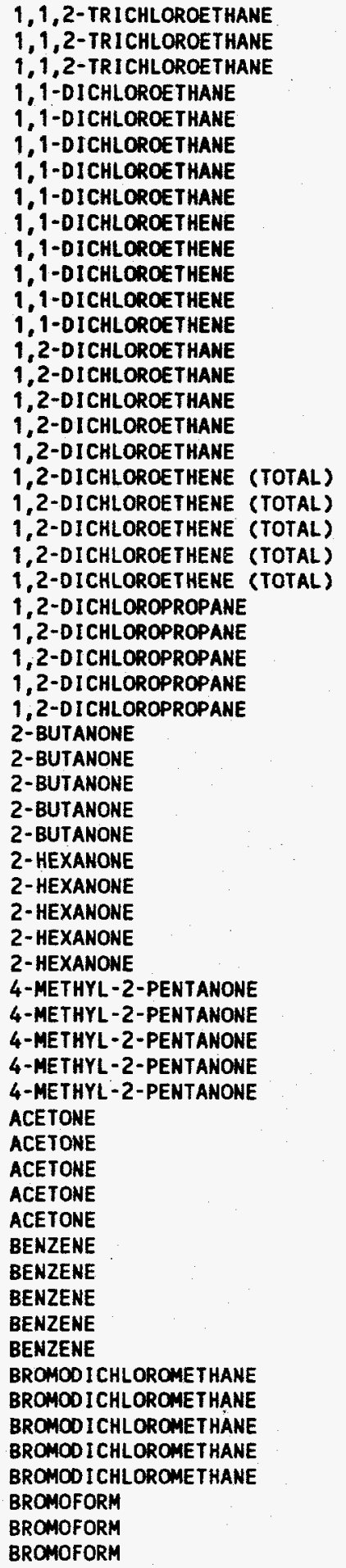 & $\begin{array}{l}\text { ND } \\
\text { ND } \\
\text { ND } \\
\text { ND } \\
\text { ND } \\
\text { ND } \\
\text { ND } \\
\text { ND } \\
\text { ND } \\
\text { ND } \\
\text { ND } \\
\text { ND } \\
\text { ND } \\
\text { ND } \\
\text { ND } \\
\text { ND } \\
\text { ND } \\
\text { ND } \\
\text { ND } \\
\text { ND } \\
\text { ND } \\
\text { ND } \\
\text { ND } \\
\text { ND } \\
\text { ND } \\
\text { ND } \\
\text { ND } \\
\text { ND } \\
\text { ND } \\
\text { ND } \\
\text { ND } \\
\text { ND } \\
\text { ND } \\
\text { ND } \\
\text { ND } \\
\text { ND } \\
\text { ND } \\
\text { ND } \\
\text { ND } \\
\text { ND } \\
\text { ND } \\
\text { ND } \\
\text { ND } \\
\text { ND } \\
\text { ND } \\
\text { ND } \\
\text { ND } \\
\text { ND } \\
\text { ND } \\
\text { ND } \\
\text { ND } \\
\text { ND } \\
\text { ND } \\
\text { ND } \\
\text { ND } \\
\text { ND } \\
\text { ND } \\
\text { ND } \\
\text { ND } \\
\text { ND } \\
\text { ND }\end{array}$ & $\begin{array}{l}10 \\
10 \\
10 \\
10.0 \\
10 \\
10 \\
10 \\
10 \\
10.0 \\
10 \\
10 \\
10 \\
10 \\
10.0 \\
10 \\
10 \\
10 \\
10 \\
10.0 \\
10 \\
10 \\
10 \\
10 \\
10.0 \\
10 \\
10 \\
10 \\
10 \\
10.0 \\
10 \\
10 \\
10 \\
10 \\
10.0 \\
10 \\
10 \\
10 \\
10 \\
10.0 \\
10 \\
10 \\
10 \\
10 \\
10.0 \\
10 \\
10 \\
10 \\
10 \\
10.0 \\
10 \\
10 \\
10 \\
10 \\
10.0 \\
10 \\
10 \\
10 \\
10 \\
10.0 \\
10 \\
10 \\
\\
10 \\
\end{array}$ & $\begin{array}{l}\text { UG/L } \\
U G / L \\
U G / L \\
U G / L \\
U G / L \\
U G / L \\
U G / L \\
U G / L \\
U G / L \\
U G / L \\
U G / L \\
U G / L \\
U G / L \\
U G / L \\
U G / L \\
U G / L \\
U G / L \\
U G / L \\
U G / L \\
U G / L \\
U G / L \\
U G / L \\
U G / L \\
U G / L \\
U G / L \\
U G / L \\
U G / L \\
U G / L \\
U G / L \\
U G / L \\
U G / L \\
U G / L \\
U G / L \\
U G / L \\
U G / L \\
U G / L \\
U G / L \\
U G / L \\
U G / L \\
U G / L \\
U G / L \\
U G / L \\
U G / L \\
U G / L \\
U G / L \\
U G / L \\
U G / L \\
U G / L \\
U G / L \\
U G / L \\
U G / L \\
U G / L \\
U G / L \\
U G / L \\
U G / L \\
U G / L \\
U G / L \\
U G / L \\
U G / L \\
U G / L \\
U G / L\end{array}$ & 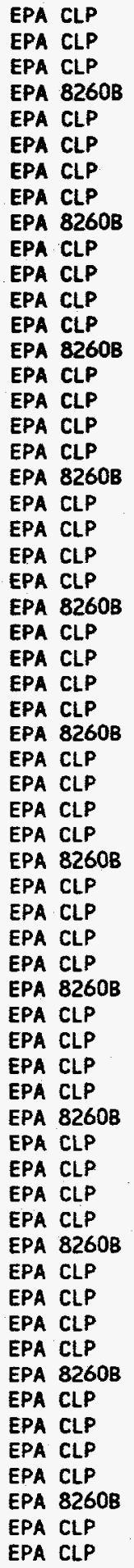 \\
\hline
\end{tabular}




\begin{tabular}{|c|c|c|c|c|c|c|c|}
\hline WSSRAP_ID & DATE_SAM & PARAMETER & CONC & DL & UNITS & METHOD & \\
\hline 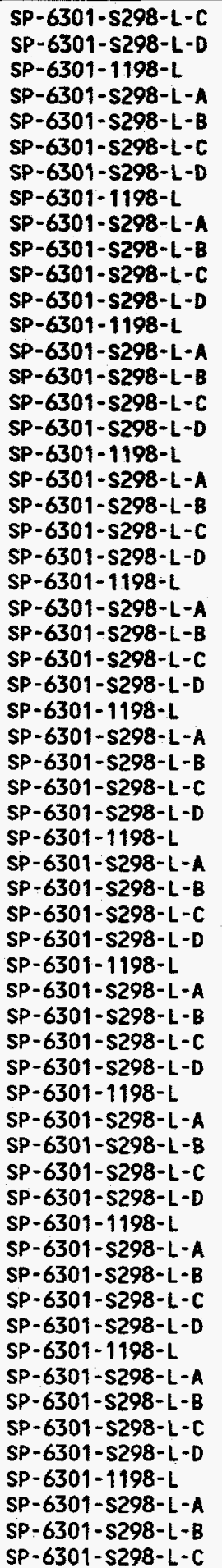 & $\begin{array}{l}12 / 12 / 98 \\
12 / 12 / 98 \\
11 / 18 / 98 \\
12 / 12 / 98 \\
12 / 12 / 98 \\
12 / 12 / 98 \\
12 / 12 / 98 \\
11 / 18 / 98 \\
12 / 12 / 98 \\
12 / 12 / 98 \\
12 / 12 / 98 \\
12 / 12 / 98 \\
11 / 18 / 98 \\
12 / 12 / 98 \\
12 / 12 / 98 \\
12 / 12 / 98 \\
12 / 12 / 98 \\
11 / 18 / 98 \\
12 / 12 / 98 \\
12 / 12 / 98 \\
12 / 12 / 98 \\
12 / 12 / 98 \\
11 / 18 / 98 \\
12 / 12 / 98 \\
12 / 12 / 98 \\
12 / 12 / 98 \\
12 / 12 / 98 \\
11 / 18 / 98 \\
12 / 12 / 98 \\
12 / 12 / 98 \\
12 / 12 / 98 \\
12 / 12 / 98 \\
11 / 18 / 98 \\
12 / 12 / 98 \\
12 / 12 / 98 \\
12 / 12 / 98 \\
12 / 12 / 98 \\
11 / 18 / 98 \\
12 / 12 / 98 \\
12 / 12 / 98 \\
12 / 12 / 98 \\
12 / 12 / 98 \\
11 / 18 / 98 \\
12 / 12 / 98 \\
12 / 12 / 98 \\
12 / 12 / 98 \\
12 / 12 / 98 \\
11 / 18 / 98 \\
12 / 12 / 98 \\
12 / 12 / 98 \\
12 / 12 / 98 \\
12 / 12 / 98 \\
11 / 18 / 98 \\
12 / 12 / 98 \\
12 / 12 / 98 \\
12 / 12 / 98 \\
12 / 12 / 98 \\
11 / 18 / 98 \\
12 / 12 / 98 \\
12 / 12 / 98 \\
12 / 12 / 98\end{array}$ & $\begin{array}{l}\text { BROMOFORM } \\
\text { BROMOFORM } \\
\text { BROMOMETHANE } \\
\text { BROMOMETHANE } \\
\text { BROMOMETHANE } \\
\text { BROMOMETHANE } \\
\text { BROMONETHANE } \\
\text { CARBON DISULFIDE } \\
\text { CARBON DISULFIDE } \\
\text { CARBON DISULFIDE } \\
\text { CARBON DISULFIDE } \\
\text { CARBON DI SULFIDE } \\
\text { CARBON TETRACHLORIDE } \\
\text { CARBON TETRACHLORIDE } \\
\text { CARBON TETRACHLORIDE } \\
\text { CARBON TETRACHLORIDE } \\
\text { CARBOH TETRACHLORIDE } \\
\text { CHLOROBENZENE } \\
\text { CHLOROBENZENE } \\
\text { CHLOROBENZENE } \\
\text { CHLOROBENZENE } \\
\text { CHLOROBENZENE } \\
\text { CHLOROETHANE } \\
\text { CHLOROETHANE } \\
\text { CHLOROETHANE } \\
\text { CHLOROETHANE } \\
\text { CHLOROETHANE } \\
\text { CHLOROFORM } \\
\text { CHLOROFORM } \\
\text { CHLOROFORM } \\
\text { CHLOROFORM } \\
\text { CHLOROFORM } \\
\text { CHLOROMETHANE } \\
\text { CHLORONETHANE } \\
\text { CHLORONETHANE } \\
\text { CHLOROMETHANE } \\
\text { CHLOROMETHANE } \\
\text { CIS- } 1,3-D I C H L O R O P R O P E N E ~ \\
\text { CIS-1,3-DICHLOROPROPENE } \\
\text { CIS-1,3-DICHLOROPROPENE } \\
\text { CIS-1,3-DICHLOROPROPENE } \\
\text { CIS-1,3-DICHLOROPROPENE } \\
\text { DIBROMOCHLOROMETHANE } \\
\text { DIBROMOCHLOROMETHANE } \\
\text { DIBRONOCHLOROMETHANE } \\
\text { DIBRONOCHLOROMETHANE } \\
\text { DIBROMOCHLOROMETHANE } \\
\text { ETHYL BENZENE } \\
\text { ETHYL BENZENE } \\
\text { ETHYL BENZENE } \\
\text { ETHYL BENZENE } \\
\text { ETHYL BENZENE } \\
\text { METHYLENE CHLORIDE } \\
\text { METHYLENE CHLOR IDE } \\
\text { METHYLENE CHLORIDE } \\
\text { METHYLENE CHLOR IDE } \\
\text { METHYLENE CHLOR IDE } \\
\text { STYRENE } \\
\text { STYRENE } \\
\text { STYRENE } \\
\text { STYRENE }\end{array}$ & $\begin{array}{l}\text { ND } \\
\text { ND } \\
\text { ND } \\
\text { ND } \\
\text { ND } \\
\text { ND } \\
\text { ND } \\
\text { ND } \\
\text { ND } \\
\text { ND } \\
\text { ND } \\
\text { ND } \\
\text { ND } \\
\text { ND } \\
\text { ND } \\
\text { ND } \\
\text { ND } \\
\text { ND } \\
\text { ND } \\
\text { ND } \\
\text { ND } \\
\text { ND } \\
\text { ND } \\
\text { ND } \\
\text { ND } \\
\text { ND } \\
\text { ND } \\
\text { ND } \\
\text { ND } \\
\text { ND } \\
\text { ND } \\
\text { ND } \\
\text { ND } \\
\text { ND } \\
\text { ND } \\
\text { ND } \\
\text { ND } \\
\text { ND } \\
\text { ND } \\
\text { ND } \\
\text { ND } \\
\text { ND } \\
\text { ND } \\
\text { ND } \\
\text { ND } \\
\text { ND } \\
\text { ND } \\
\text { ND } \\
\text { ND } \\
\text { N.5 } \\
\text { ND } \\
\text { ND } \\
\text { ND } \\
\text { ND }\end{array}$ & $\begin{array}{l}10 \\
10 \\
10.0 \\
10 \\
10 \\
10 \\
10 \\
10.0 \\
10 \\
10 \\
10 \\
10 \\
10.0 \\
10 \\
10 \\
10 \\
10 \\
10.0 \\
10 \\
10 \\
10 \\
10 \\
10.0 \\
10 \\
10 \\
10 \\
10 \\
10.0 \\
10 \\
10 \\
10 \\
10 \\
10.0 \\
10 \\
10 \\
10 \\
10 \\
10.0 \\
10 \\
10 \\
10 \\
10 \\
10.0 \\
10 \\
10 \\
10 \\
10 \\
10.0 \\
10 \\
10 \\
10 \\
10 \\
10.0 \\
10 \\
10 \\
10 \\
10 \\
10.0 \\
10 \\
10 \\
10 \\
10 \\
10 \\
10 \\
10 \\
10 \\
10 \\
10 \\
10 \\
10 \\
10 \\
10 \\
10 \\
10 \\
10 \\
10\end{array}$ & 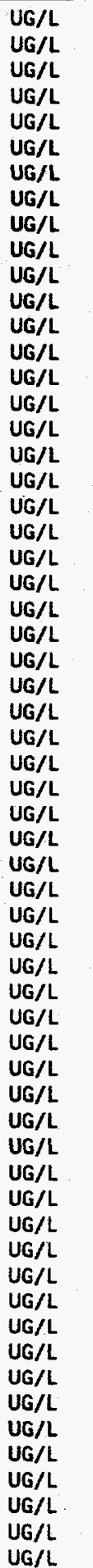 & 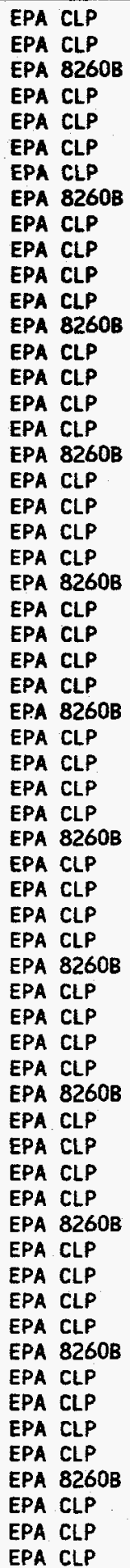 & . \\
\hline
\end{tabular}




\begin{tabular}{|c|c|c|c|c|c|c|}
\hline HSSRAP_ID & DATE_SAM & PARAMETER & conc & DL & UNITS & METHOD \\
\hline 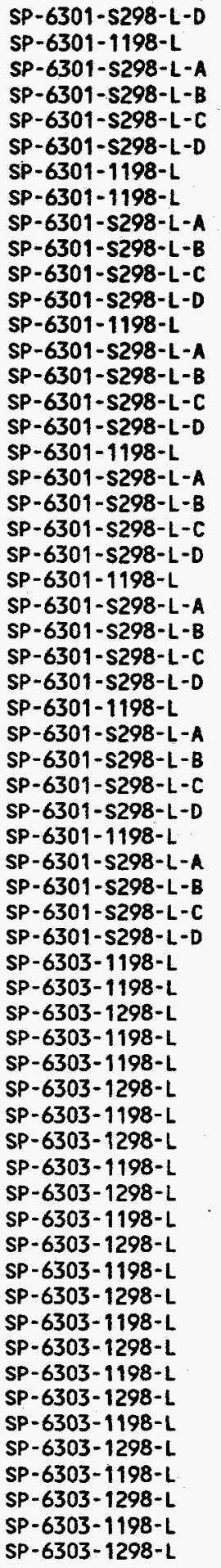 & $\begin{array}{l}12 / 12 / 98 \\
11 / 18 / 98 \\
12 / 12 / 98 \\
12 / 12 / 98 \\
12 / 12 / 98 \\
12 / 12 / 98 \\
11 / 18 / 98 \\
11 / 18 / 98 \\
12 / 12 / 98 \\
12 / 12 / 98 \\
12 / 12 / 98 \\
12 / 12 / 98 \\
11 / 18 / 98 \\
12 / 12 / 98 \\
12 / 12 / 98 \\
12 / 12 / 98 \\
12 / 12 / 98 \\
11 / 18 / 98 \\
12 / 12 / 98 \\
12 / 12 / 98 \\
12 / 12 / 98 \\
12 / 12 / 98 \\
11 / 18 / 98 \\
12 / 12 / 98 \\
12 / 12 / 98 \\
12 / 12 / 98 \\
12 / 12 / 98 \\
11 / 18 / 98 \\
12 / 12 / 98 \\
12 / 12 / 98 \\
12 / 12 / 98 \\
12 / 12 / 98 \\
11 / 18 / 98 \\
12 / 12 / 98 \\
12 / 12 / 98 \\
12 / 12 / 98 \\
12 / 12 / 98 \\
11 / 18 / 98 \\
11 / 18 / 98 \\
12 / 14 / 98 \\
11 / 18 / 98 \\
11 / 18 / 98 \\
12 / 14 / 98 \\
111 / 18 / 98 \\
12 / 14 / 98 \\
11 / 18 / 98 \\
12 / 14 / 98 \\
11 / 18 / 98 \\
12 / 14 / 98 \\
11 / 18 / 98 \\
12 / 14 / 98 \\
11 / 18 / 98 \\
12 / 14 / 98 \\
11 / 18 / 98 \\
12 / 14 / 98 \\
11 / 18 / 98 \\
12 / 14 / 98 \\
111 / 18 / 98 \\
12 / 14 / 98 \\
11 / 18 / 98 \\
12 / 14 / 98\end{array}$ & 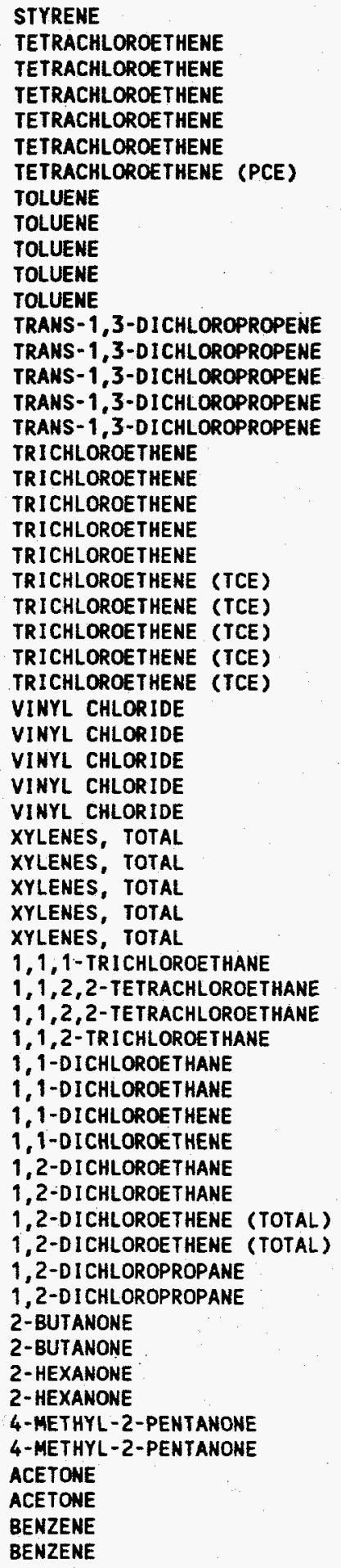 & $\begin{array}{l}\text { ND } \\
\text { ND } \\
\text { ND } \\
\text { ND } \\
\text { ND } \\
\text { ND } \\
\text { ND } \\
\text { ND } \\
\text { ND } \\
\text { ND } \\
\text { ND } \\
\text { ND } \\
\text { ND } \\
\text { ND } \\
\text { ND } \\
\text { ND } \\
\text { ND } \\
\text { ND } \\
\text { ND } \\
\text { ND } \\
\text { ND } \\
\text { ND } \\
\text { ND } \\
\text { ND } \\
\text { ND } \\
\text { ND } \\
\text { ND } \\
\text { ND } \\
\text { ND } \\
\text { ND } \\
\text { ND } \\
\text { ND } \\
\text { ND } \\
\text { ND } \\
\text { ND } \\
\text { ND } \\
\text { ND } \\
\text { ND } \\
\text { ND } \\
\text { ND } \\
\text { ND } \\
\text { ND } \\
\text { ND } \\
\text { ND } \\
\text { ND } \\
\text { ND } \\
\text { ND } \\
\text { ND } \\
\text { ND } \\
\text { ND } \\
\text { ND } \\
\text { ND } \\
\text { ND } \\
\text { ND } \\
\text { ND } \\
\text { ND } \\
\text { ND } \\
\text { ND } \\
\text { ND } \\
\text { ND } \\
\text { ND }\end{array}$ & 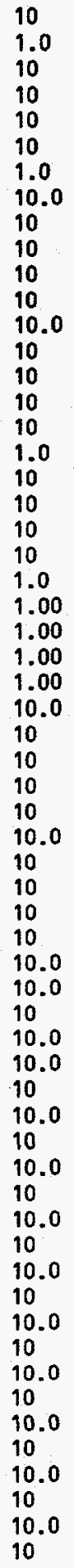 & $\begin{array}{l}\text { UG/L } \\
U G / L \\
U G / L \\
U G / L \\
U G / L \\
U G / L \\
U G / L \\
U G / L \\
U G / L \\
U G / L \\
U G / L \\
U G / L \\
U G / L \\
U G / L \\
U G / L \\
U G / L \\
U G / L \\
U G / L \\
U G / L \\
U G / L \\
U G / L \\
U G / L \\
U G / L \\
U G / L \\
U G / L \\
U G / L \\
U G / L \\
U G / L \\
U G / L \\
U G / L \\
U G / L \\
U G / L \\
U G / L \\
U G / L \\
U G / L \\
U G / L \\
U G / L \\
U G / L \\
U G / L \\
U G / L \\
U G / L \\
U G / L \\
U G / L \\
U G / L \\
U G / L \\
U G / L \\
U G / L \\
U G / L \\
U G / L \\
U G / L \\
U G / L \\
U G / L \\
U G / L \\
U G / L \\
U G / L \\
U G / L \\
U G / L \\
U G / L \\
U G / L \\
U G / L \\
U G / L \\
\text { UG } \\
\text { U. }\end{array}$ & 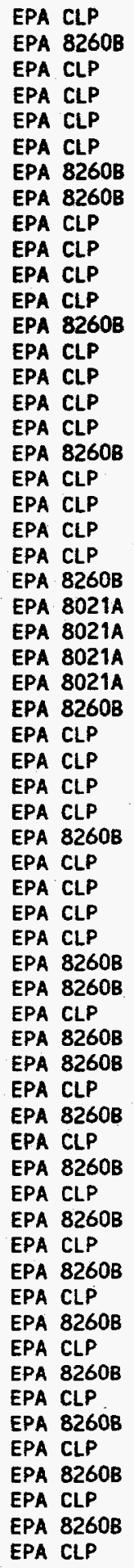 \\
\hline
\end{tabular}




\begin{tabular}{|c|c|c|c|c|c|c|}
\hline WSSRAP_10 & DATE_SAM & PARAMETER & CONC & DL & UNITS & METHOD \\
\hline 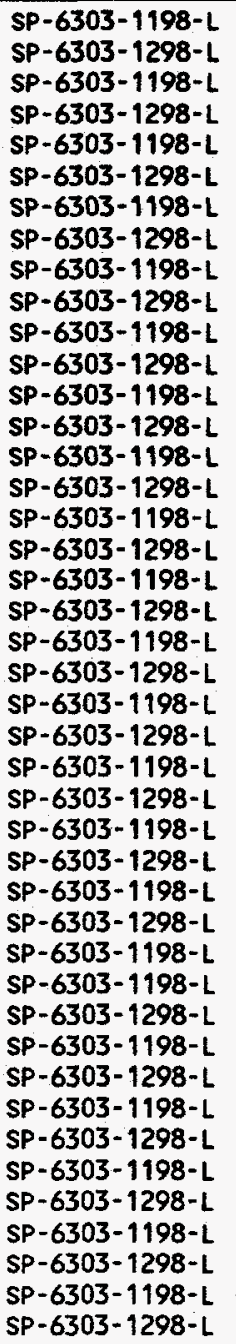 & $\begin{array}{l}11 / 18 / 98 \\
12 / 14 / 98 \\
11 / 18 / 98 \\
12 / 14 / 98 \\
11 / 18 / 98 \\
12 / 14 / 98 \\
11 / 18 / 98 \\
12 / 14 / 98 \\
11 / 18 / 98 \\
12 / 14 / 98 \\
11 / 18 / 98 \\
12 / 14 / 98 \\
11 / 18 / 98 \\
12 / 14 / 98 \\
11 / 18 / 98 \\
12 / 14 / 98 \\
11 / 18 / 98 \\
12 / 14 / 98 \\
11 / 18 / 98 \\
12 / 14 / 98 \\
11 / 18 / 98 \\
12 / 14 / 98 \\
11 / 18 / 98 \\
12 / 14 / 98 \\
11 / 18 / 98 \\
12 / 14 / 98 \\
11 / 18 / 98 \\
12 / 14 / 98 \\
11 / 18 / 98 \\
12 / 14 / 98 \\
11 / 18 / 98 \\
11 / 18 / 98 \\
12 / 14 / 98 \\
11 / 18 / 98 \\
12 / 14 / 98 \\
11 / 18 / 98 \\
12 / 14 / 98 \\
11 / 18 / 98 \\
12 / 14 / 98 \\
11 / 18 / 98 \\
12 / 14 / 98 \\
11 / 18 / 98 \\
12 / 14 / 98\end{array}$ & 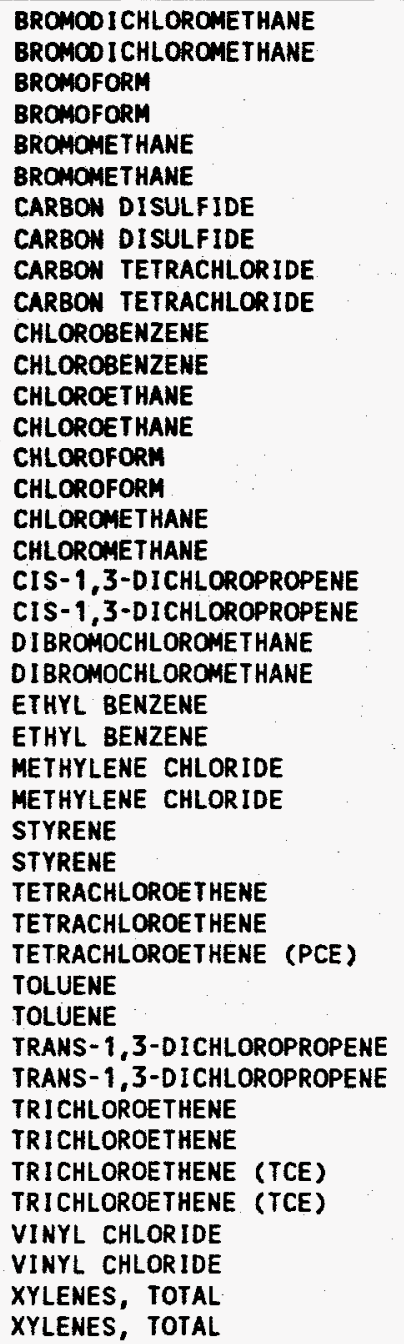 & $\begin{array}{l}\text { ND } \\
\text { ND } \\
\text { ND } \\
\text { ND } \\
\text { ND } \\
\text { ND } \\
\text { ND } \\
\text { ND } \\
\text { ND } \\
\text { ND } \\
\text { ND } \\
\text { ND } \\
\text { ND } \\
\text { ND } \\
\text { ND } \\
\text { ND } \\
\text { ND } \\
\text { ND } \\
\text { ND } \\
\text { ND } \\
\text { ND } \\
\text { ND } \\
\text { (3) } \\
\text { ND } \\
\text { ND } \\
\text { ND } \\
\text { ND } \\
\text { ND } \\
\text { ND } \\
\text { ND } \\
\text { ND } \\
\text { ND } \\
1.1 \\
\text { ND } \\
1.1 \\
\text { ND } \\
\text { ND } \\
\text { ND } \\
\text { ND } \\
\text { ND }\end{array}$ & $\begin{array}{l}10.0 \\
10 \\
10.0 \\
10 \\
10.0 \\
10 \\
10.0 \\
10 \\
10.0 \\
10 \\
10.0 \\
10 \\
10.0 \\
10 \\
10.0 \\
10 \\
10.0 \\
10 \\
10.0 \\
10 \\
10.0 \\
10 \\
10.0 \\
10 \\
10.0 \\
10 \\
10.0 \\
10 \\
1.0 \\
10 \\
1.0 \\
10.0 \\
10 \\
10.0 \\
10 \\
1.0 \\
10 \\
1.0 \\
1 \\
10.0 \\
10 \\
10.0 \\
10 \\
\\
\\
\\
\\
\end{array}$ & $\begin{array}{l}U G / L \\
U G / L \\
U G / L \\
U G / L \\
U G / L \\
U G / L \\
U G / L \\
U G / L \\
U G / L \\
U G / L \\
U G / L \\
U G / L \\
U G / L \\
U G / L \\
U G / L \\
U G / L \\
U G / L \\
U G / L \\
U G / L \\
U G / L \\
U G / L \\
U G / L \\
U G / L \\
U G / L \\
U G / L \\
U G / L \\
U G / L \\
U G / L \\
U G / L \\
U G / L \\
U G / L \\
U G / L \\
U G / L \\
U G / L \\
U G / L \\
U G / L \\
U G / L \\
U G / L \\
U G / L \\
U G / L \\
U G / L \\
U G / L \\
U G / L\end{array}$ & 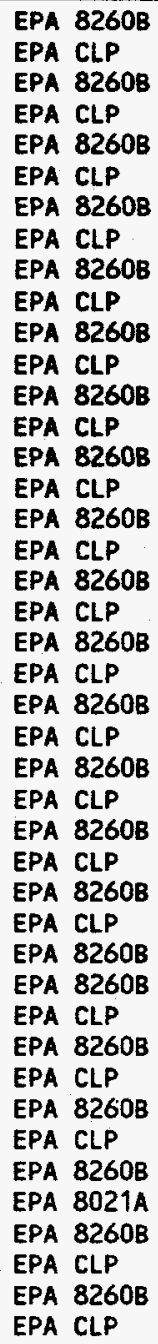 \\
\hline
\end{tabular}




\section{AIR MONITORING}




\section{First Quarter 1999 Radioactive Airborne Particulate Results}

$\begin{array}{cccc}\text { LOCATION ID } & \begin{array}{c}\text { QUARTERLY AVERAGE GROSS } \\ \text { ALPHA CONCENTRATION }(\mu \mathrm{Ci} / \mathrm{ml})\end{array} & \begin{array}{c}\text { STANDARD } \\ \text { DEVIATION }\end{array} & \begin{array}{c}\text { \# WEEKS } \\ \text { COLLECTED }\end{array} \\ \text { AP-1009 } & 1.08 \mathrm{E}-15 & 3.03 \mathrm{E}-16 & 13 \\ \text { AP-1017 } & 1.06 \mathrm{E}-15 & 2.67 \mathrm{E}-16 & 13 \\ \text { AP-2001 } & 1.17 \mathrm{E}-15 & 3.54 \mathrm{E}-16 & 13 \\ \text { AP-2002 } & 1.46 \mathrm{E}-15 & 4.62 \mathrm{E}-16 & 13 \\ \text { AP-2005 } & 1.31 \mathrm{E}-15 & 4.77 \mathrm{E}-16 & 13 \\ \text { AP-2008 } & 1.28 \mathrm{E}-15 & 4.81 \mathrm{E}-16 & 13 \\ \text { AP-2013 } & 1.32 \mathrm{E}-15 & 4.65 \mathrm{E}-16 & 5 \\ \text { AP-2025 } & 1.32 \mathrm{E}-15 & 5.23 \mathrm{E}-16 & 13 \\ \text { AP-3003 } & 1.55 \mathrm{E}-15 & 7.32 \mathrm{E}-16 & 13 \\ \text { AP-3004 } & 2.13 \mathrm{E}-15 & 1.87 \mathrm{E}-15 & 13 \\ \text { AP-3014 } & 1.50 \mathrm{E}-15 & 7.40 \mathrm{E}-16 & 13 \\ \text { AP-4006 } & 1.13 \mathrm{E}-15 & 3.17 \mathrm{E}-16 & 13 \\ \text { AP-4007 } & 1.27 \mathrm{E}-15 & 2.80 \mathrm{E}-16 & 13 \\ \text { AP-4008 } & 1.35 \mathrm{E}-15 & 3.40 \mathrm{E}-16 & 13 \\ \text { AP-4011 } & 1.21 \mathrm{E}-15 & 3.37 \mathrm{E}-16 & 13 \\ \text { AP-4012* } & 1.15 \mathrm{E}-15 & 4.56 \mathrm{E}-16 & 13 \\ \text { AP-4013 } & 9.49 \mathrm{E}-16 & 3.86 \mathrm{E}-16 & 6 \\ \text { AP-4014 } & 9.66 \mathrm{E}-16 & 2.22 \mathrm{E}-16 & \\ \text { * } & & & \\ \text { * Background monitoring location } & & & \\ \text { 52-week Background Concentration: } & .1 .26 \mathrm{E}-15 \mu \mathrm{Ci} / \mathrm{ml} & & \end{array}$




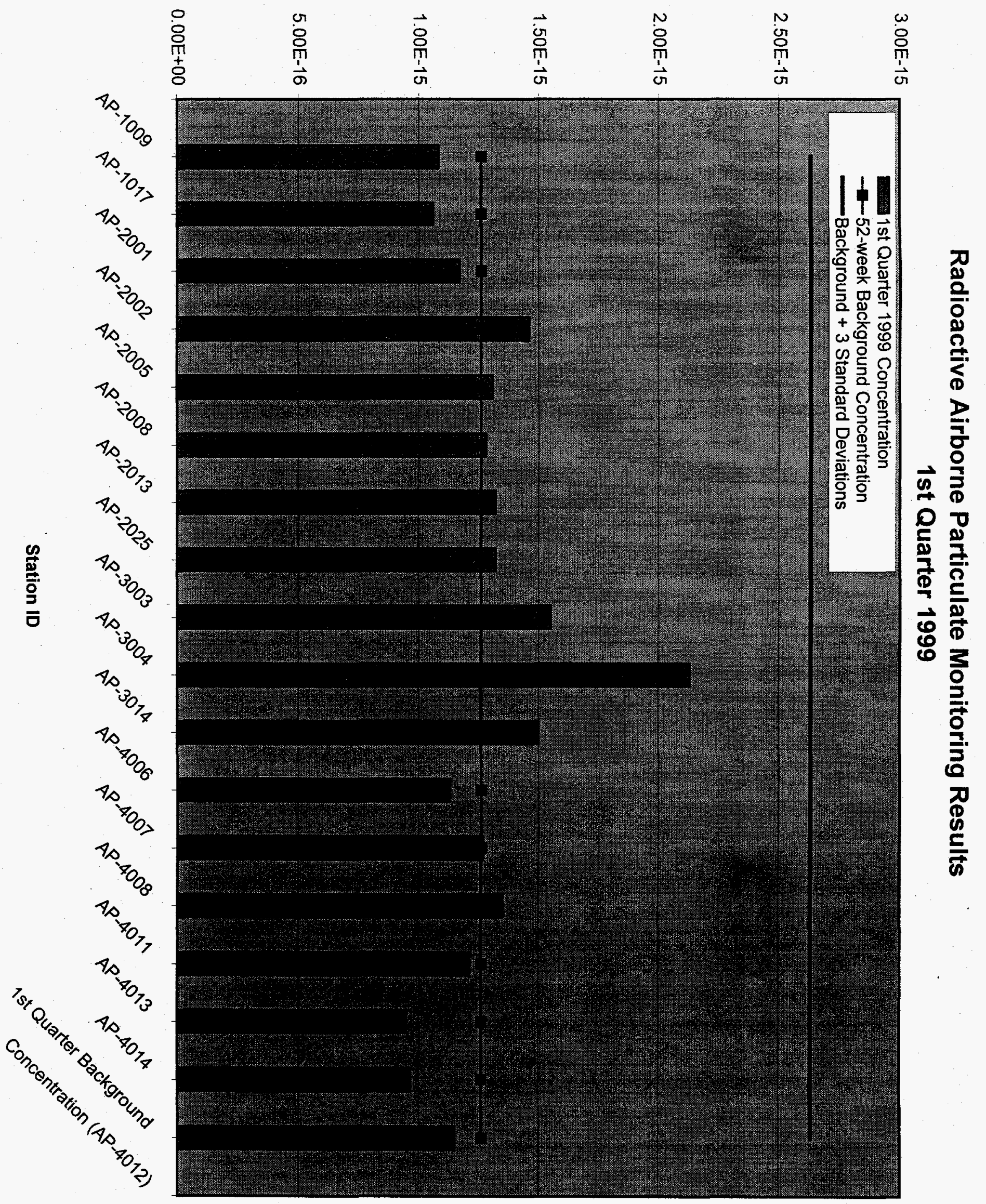


Fourth Quarter 1998 Alpha Track Radon Monitoring Results

LOCATION ID

AVERAGE (pCi/L)

PERCENT OF DCG(a)

Weldon Spring Ouarry

RD-1002

0.1

0

RD-1005

0.2

3

Weldon Spring Chemical Plant

RD-2002

0.1

0

$\mathrm{RD}-2004$

0.2

0.2

$\mathrm{RD}-2006$

$\mathrm{RD}-2007$

0.2

$\mathrm{RD}-2025$

0.2

WSSRAP Disposal Facility

RD-CE01

0.1

0

$\mathrm{RD}-\mathrm{CE} 02$

0.1

0

RD-CE05

0.2

3

RD-CE06

0.4

10

Weldon Spring Raffinate Pits

RD-3001
RD-3002
RD-3003
RD-3004
RD-3005
RD-3008
$R D-3009$
$R D-3013$
$R D-3016$
$R D-3017$
$R D-3018$
$R D-3019$

0.2

0.2

3

0.1

0.1

0.1

$-$

0.2

0.4

0.1

0.3

0.1

0.2

3

0

0

0

RD-3019

\section{Off Site}

RD-4001

0.2

0.1

3

RD-4002

0.1

RD-4003

0.2

$\mathrm{RD}-4005^{(\mathrm{b})}$

0.2

RD-4009 (b)

0.1

0.1

RD-4013

0.2

3

0

7

0

3

52-week Background Average

3
0
0
N/A
3
N/A
0
N/A

(a) Derived Concentration Guide. Percent of the DCG is determined by subtracting the combined 52-week background average from a station's reported quarterly concentration, dividing by $3 \mathrm{pCi} / \mathrm{L}$ (the $\mathrm{DCG}$ for radon), and multiplying by 100 .

(b) Denotes background monitoring location.

-. Missing detector. 
Fourth Quarter 1998 Alpha Track Thoron Monitoring Results

LOCATION ID

$\operatorname{AVERAGE}(\mathrm{pCi} / \mathrm{L}) \quad$ PERCENT OF DCG ${ }^{(\mathrm{a})}$

Weldon Spring Quarry

RD-1002

0.2

3

RD-1005

0.1

0

Weldon Spring Chemical Plant

RD-2002

0.2

3

$\mathrm{RD}-2004$

0.0

0

RD-2006

0.0

0

RD-2007

0.0

0

RD-2025

0.3

7

WSSRAP Disposal Facility

RD-CE01

0.8

23

RD-CE02

0.8

23

RD-CE05

0.6

17

RD-CE06

0.8

23

Weldon Spring Raffinate Pits

RD-3001

0.2

3

RD-3002

0.5

13

RD-3003

0.6

17

$\mathrm{RD}-3004$

0.0

0

$\mathrm{RD}-3005$

0.2

3

RD-3008

RD-3009

$--$

RD-3013

0.5

N/A

RD-3016

3.2

13

RD-3017

0.2

103

RD-3018

0.1

3

0.3

0

RD-3019

8.2

7

270

\section{Off Site}

RD-4001

0.0

0.1

RD-4002

0.0

RD-4003

RD-4005 ${ }^{(b)}$

0.0

RD-4007

0.0

RD-4009 ${ }^{(b)}$

0.1

0.1

RD-4013

0.0

52-Week Background Average

0
0
0
N/A
0
N/A
0
N/A

(a) Derived Concentration Guide. Percent of the DCG is determined by subtracting the combined 52-week background average from a station's reported quarterly concentration, dividing by $3 \mathrm{pCi} / \mathrm{L}$ (the $\mathrm{DCG}$ for thoron), and multiplying by 100 .

(b) Denotes i ackground monitoring location.

-- Missing detector. 


\section{Alpha Track Radon Monitoring Results}

4th Quarter 1998

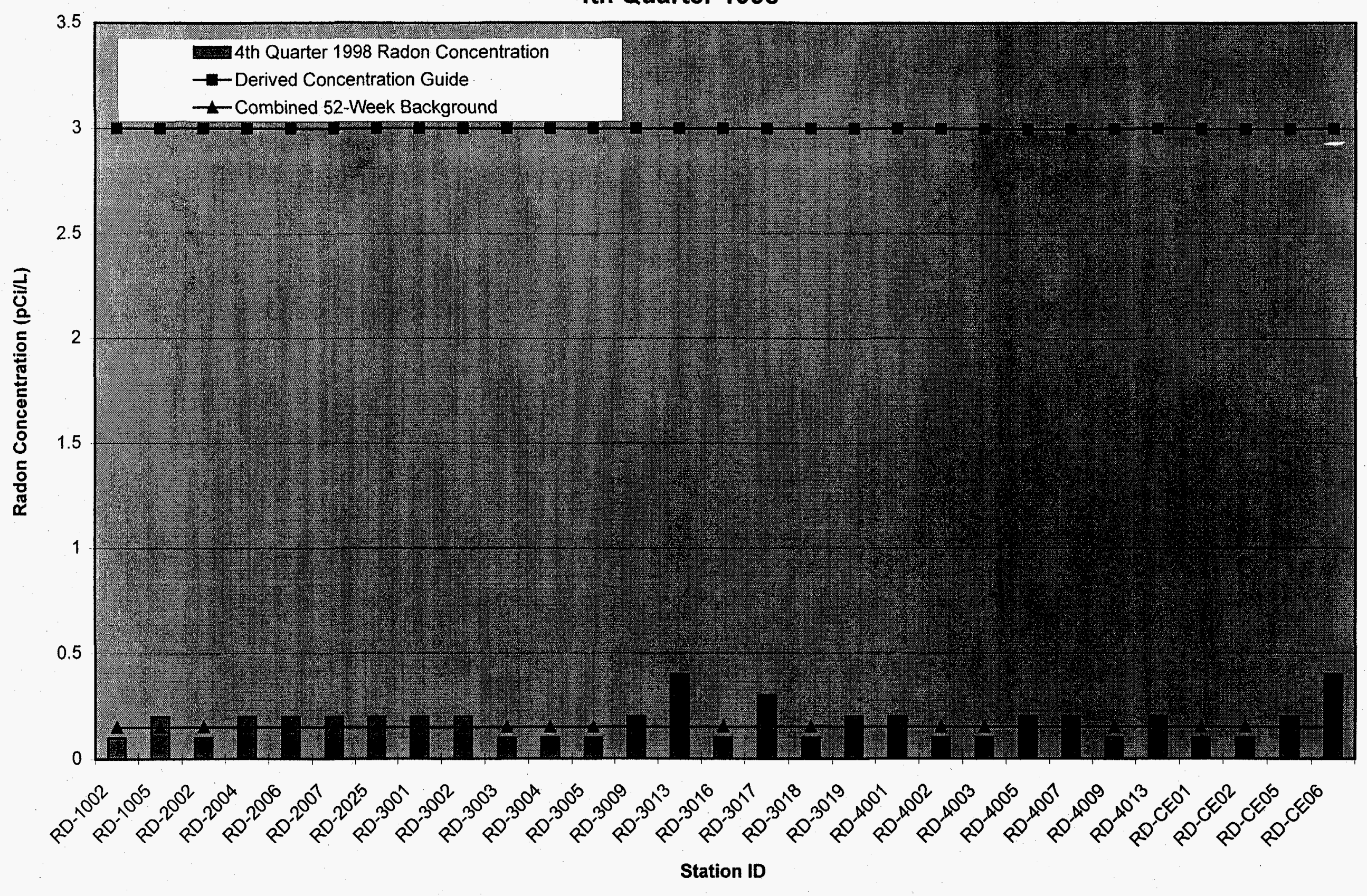




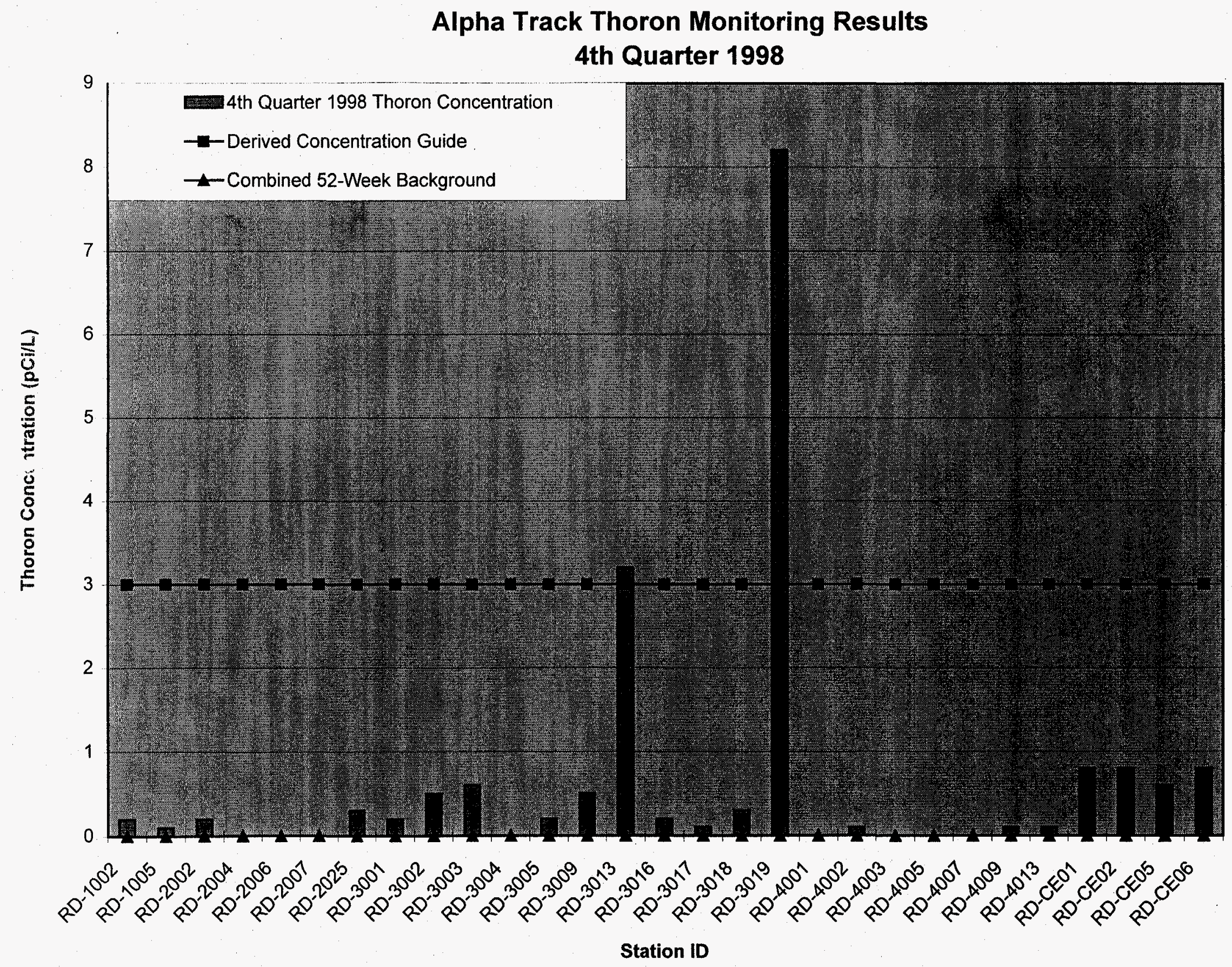


FOURTH QUARTER 1998 ENVIRONMENTAL TLD RESULTS

\begin{tabular}{||c|c|}
\hline \multicolumn{1}{|c|}{ STATION } & RESULTS (mrem) \\
\hline \multicolumn{2}{|c|}{ Weldon Spring Quarry } \\
\hline TD-1002 & 15.0 \\
\hline \multicolumn{2}{|c|}{ Weldon Spring Chemical Plant } \\
\hline TD-2004 & 16.6 \\
TD-2006 & 15.7 \\
TD-2025 & 15.8 \\
\hline Weldon Spring Raffinate Pits \\
\hline TD-3001 & 16.2 \\
TD-3002 & 21.3 \\
TD-3003 & 20.9 \\
TD-3004 & 15.9 \\
\hline \multicolumn{2}{|c|}{} \\
\hline TD-4001 & 15.2 \\
TD-4002 & 13.1 \\
TD-4003 & 12.7 \\
TD-4005* & 14.2 \\
TD-4007 & 15.3 \\
TD-4009* & 14.8 \\
TD-4013 & 15.1 \\
\hline
\end{tabular}

* Background monitoring station 


\section{Environmental TLD Monitoring Results}

4th Quarter 1998

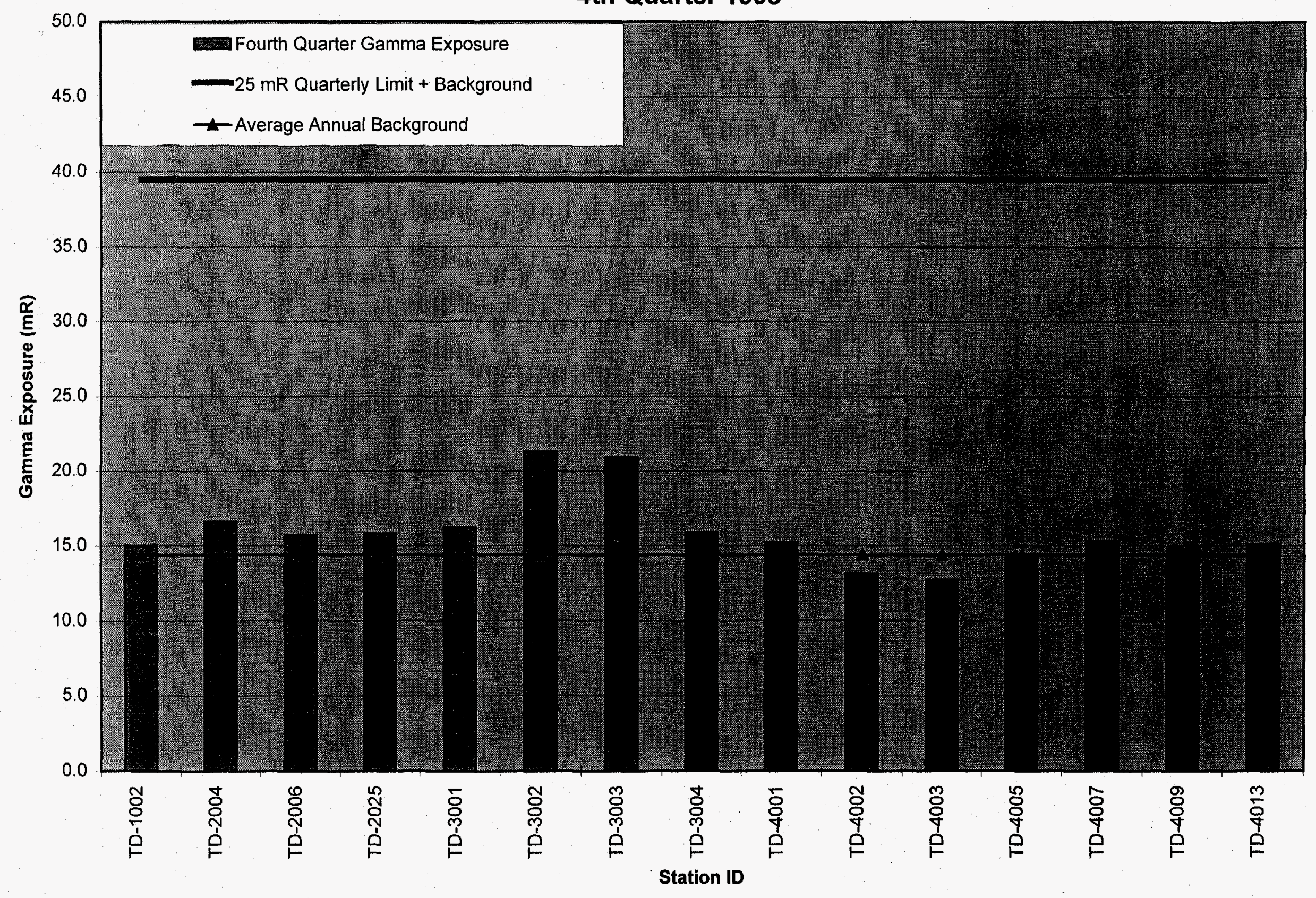

